\title{
EXCLUSIVE B DECAYS INTO FINAL STATES WITH TWO CHARMED
}

\section{BARYONS}

by

Xuedong Chai

\begin{abstract}
$\underline{\text { An Abstract }}$
Of a thesis submitted in partial fulfillment of the requirements for the Doctor of Philosophy degree in Physics in the Graduate College of The University of Iowa
\end{abstract}

December 2007

Thesis Supervisor: Professor Usha Mallik 


\begin{abstract}
This Thesis presents measurements of the decays $B^{-} \rightarrow \Xi_{c}^{0} \bar{\Lambda}_{c}^{-}, \bar{B}^{0} \rightarrow \Xi_{c}^{+} \bar{\Lambda}_{c}^{-}$, $B^{-} \rightarrow \Lambda_{c}^{+} \bar{\Lambda}_{c}^{-} K^{-}$, and $\bar{B}^{0} \rightarrow \Lambda_{c}^{+} \bar{\Lambda}_{c}^{-} K_{s}^{0}$ based on 228 million $\Upsilon(4 S) \rightarrow B \bar{B}$ decays collected with the BABAR detector at the SLAC PEP-II asymmetric-energy $B$ factory. The branching fraction of $B^{-} \rightarrow \Lambda_{c}^{+} \bar{\Lambda}_{c}^{-} K^{-}$is measured to be $(1.22 \pm 0.16 \pm 0.14 \pm$ $0.63) \times 10^{-3}$, and the branching fraction of $B^{0} \rightarrow \Lambda_{c}^{+} \bar{\Lambda}_{c}^{-} \bar{K}^{0}$ is $(0.40 \pm 0.33 \pm 0.05 \pm$ $0.21) \times 10^{-3}$. The product $\mathcal{B}\left(B^{-} \rightarrow \Xi_{c}^{0} \bar{\Lambda}_{c}^{-}\right) \times \mathcal{B}\left(\Xi_{c}^{0} \rightarrow \Xi^{-} \pi^{+}\right)$is measured to be $(2.04 \pm 0.59 \pm 0.26 \pm 0.53) \times 10^{-5}$, and $\mathcal{B}\left(\bar{B}^{0} \rightarrow \Xi_{c}^{+} \bar{\Lambda}_{c}^{-}\right) \times \mathcal{B}\left(\Xi_{c}^{+} \rightarrow \Xi^{-} \pi^{+} \pi^{+}\right)$is $(1.50 \pm 1.07 \pm 0.19 \pm 0.39) \times 10^{-5}$, where the uncertainties are statistical, systematic, and the uncertainty from the branching fraction $\mathcal{B}\left(\Lambda_{c}^{+} \rightarrow p^{+} K^{-} \pi^{+}\right)$, respectively.
\end{abstract}

Abstract Approved:

Thesis Supervisor

Title and Department

Date 


\title{
EXCLUSIVE B DECAYS INTO FINAL STATES WITH TWO CHARMED BARYONS
}

\author{
by \\ Xuedong Chai
}

A thesis submitted in partial fulfillment of the requirements for the Doctor of Philosophy degree in Physics

in the Graduate College of

The University of Iowa

December 2007

Thesis Supervisor: Professor Usha Mallik 


\author{
Graduate College \\ The University of Iowa \\ Iowa City, Iowa
}

\title{
CERTIFICATE OF APPROVAL
}

\section{PH.D. THESIS}

This is to certify that the Ph.D. thesis of

\section{Xuedong Chai}

has been approved by the Examining Committee for the thesis requirement for the Doctor of Philosophy degree in Physics at the December 2007 graduation.

Thesis Committee:

Usha Mallik, Thesis Supervisor

Dong $\mathrm{Su}$

Paul Kleiber

Vincent Rodgers

William Daughton 
To my father 


\section{ACKNOWLEDGEMENTS}

To be survived with the Graduate study is not easy and painful. Without many people's help, it would not be finished forever. Here, I will express my appreciation to the people who contribute to this dissertation directly or indirectly.

First of all, I am grateful to my thesis advisor, Usha Mallik. It is she guide me into the experimental particle physics. Without her close guidance and never-ending support, the thesis work would not have been possible to be finished.

A very special thanks is owed to the advisors who steer me finish the long and painful graduate classes - William H. Klink ,Yannick Meurice, Wayne N. Polyzou, Edward R. McCliment.

In addition, I'd like to thank all the people in the Iowa group: Gerald JeanJacques Grenier, Matthew John Charles, Wolfgang F. Maders, Ajit Kumar Mohapatra, Niels Thorben Meyer, Prafulla Kumar Behera.

I'd like also thank Dong Su and Bill Dunwoodie at SLAC, who are kindly and eagerly teach me, a HEP student all their knowledge and experience. I really appreciate that.

Finally I want to thank my mother, my brothers, my wife and my son - Qing for the encouragement and support that they have given to me for many years.

I'd like to dedicate this thesis to my father, who passed away while I was in the high school. Dad, I'm sorry you could not see my graduation. 


\begin{abstract}
This Thesis presents measurements of the decays $B^{-} \rightarrow \Xi_{c}^{0} \bar{\Lambda}_{c}^{-}, \bar{B}^{0} \rightarrow \Xi_{c}^{+} \bar{\Lambda}_{c}^{-}$, $B^{-} \rightarrow \Lambda_{c}^{+} \bar{\Lambda}_{c}^{-} K^{-}$, and $\bar{B}^{0} \rightarrow \Lambda_{c}^{+} \bar{\Lambda}_{c}^{-} K_{s}^{0}$ based on 228 million $\Upsilon(4 S) \rightarrow B \bar{B}$ decays collected with the BABAR detector at the SLAC PEP-II asymmetric-energy $B$ factory. The branching fraction of $B^{-} \rightarrow \Lambda_{c}^{+} \bar{\Lambda}_{c}^{-} K^{-}$is measured to be $(1.22 \pm 0.16 \pm 0.14 \pm$ $0.63) \times 10^{-3}$, and the branching fraction of $B^{0} \rightarrow \Lambda_{c}^{+} \bar{\Lambda}_{c}^{-} \bar{K}^{0}$ is $(0.40 \pm 0.33 \pm 0.05 \pm$ $0.21) \times 10^{-3}$. The product $\mathcal{B}\left(B^{-} \rightarrow \Xi_{c}^{0} \bar{\Lambda}_{c}^{-}\right) \times \mathcal{B}\left(\Xi_{c}^{0} \rightarrow \Xi^{-} \pi^{+}\right)$is measured to be $(2.04 \pm 0.59 \pm 0.26 \pm 0.53) \times 10^{-5}$, and $\mathcal{B}\left(\bar{B}^{0} \rightarrow \Xi_{c}^{+} \bar{\Lambda}_{c}^{-}\right) \times \mathcal{B}\left(\Xi_{c}^{+} \rightarrow \Xi^{-} \pi^{+} \pi^{+}\right)$is $(1.50 \pm 1.07 \pm 0.19 \pm 0.39) \times 10^{-5}$, where the uncertainties are statistical, systematic, and the uncertainty from the branching fraction $\mathcal{B}\left(\Lambda_{c}^{+} \rightarrow p^{+} K^{-} \pi^{+}\right)$, respectively.
\end{abstract}




\section{TABLE OF CONTENTS}

LIST OF TABLES ................................ vii

LIST OF FIGURES . . . . . . . . . . . . . . . . . . . .

CHAPTER

1 INTRODUCTION . . . . . . . . . . . . . . . . . 1

1.1 Standard Model . . . . . . . . . . . . . . . . . . 1

1.1.1 Fundamental particles . . . . . . . . . . . . . . 1

1.1.2 Fundamental interactions ............... . . . . . . . 4

1.1.3 Perspectives on the Standard Model . . . . . . . . . . 9

1.2 Charmed baryons . . . . . . . . . . . . . . 10

1.2.1 A short history of the charm quark . . . . . . . . . . 10

1.2.2 Quark model of the charmed baryons . . . . . . . . . . 10

1.2.3 Decay of charmed baryons . . . . . . . . . . . . . 15

1.3 B meson physics . . . . . . . . . . . . . . . . . . . . . . 18

1.3.1 Hadronic decays of $B$ meson . . . . . . . . . . . . 19

1.3.2 Inclusive B meson decays to baryons . . . . . . . . . . . . 21

1.3.3 Exclusive B meson decays to charmed baryon pairs . . . . 22

1.4 An overview of the thesis . . . . . . . . . . . 26

2 EXPERIMENTAL FACILITY - BABAR DETECTOR AND PEP-II . 28

2.1 PEP-II accelerator . . . . . . . . . . . . . . . . . . . . . 28

2.2 BABAR detector . . . . . . . . . . . . . . . . . . . . . . . . . . . 32

2.2.1 BABAR coordinate system . . . . . . . . . . . . . . 33

2.3 Tracking system . . . . . . . . . . . . . . . . . . . . . . . . . . . . . . . . 35

2.3.1 Silicon vertex detector . . . . . . . . . . . . 35

2.3.2 Drift chamber $(\mathrm{DCH}) \ldots \ldots . \ldots . \ldots 38$

2.4 Detector for internally reflected cherenkov light . . . . . . . . . . 43

2.5 Electromagnetic calorimeter (EMC) . . . . . . . . . . . . 46

2.6 Detector for muons and neutral hadrons - IFR . . . . . . . . . . 48

3 TRIGGER SYSTEM AND TRIGGER UPGRADE . . . . . . . . . 52

3.1 Trigger system overview . . . . . . . . . . . . . . . . . . . . . 52

3.2 Level 1 trigger . . . . . . . . . . . . . . . . . . . . . . . . . . . . . . . . . . . 53

3.2.1 L1 DCH trigger . . . . . . . . . . . . . . . 54

3.2 .2 L1 EMC trigger . . . . . . . . . . . . . . . 59 
3.2 .3 L1 IFR trigger . . . . . . . . . . . . . . . . . . . . 60

$3.2 .4 \quad$ L1 global level trigger . . . . . . . . . . . . . . . 61

3.3 Level 3 trigger . . . . . . . . . . . . . . . . . . . . . . . 63

3.4 Level 1 DCT trigger upgrade . . . . . . . . . . . . . . 63

3.4.1 Design requirement . . . . . . . . . . . . . . . . . 64

3.4.2 Design implementation of the TSF modules . . . . . . . 67

3.4.3 Design implementation of $\mathrm{z}$ and $p_{t}$ discriminator (ZPD) . 67

3.4.4 Design implementation of the interface-boards . . . . . . 68

3.4.5 System testing strategy . . . . . . . . . . . . . 70

3.4.6 Performance of the complete DCT system . . . . . . . . 71

4 TRACKING, PARTICLE ID AND VERTEXING . . . . . . . . 73

4.1 Charged track reconstruction . . . . . . . . . . . . . . 73

4.2 Particle identification . . . . . . . . . . . . . . . . . 74

4.3 Kinematic fit and decay chain reconstruction . . . . . . . . 76

5 DATA DESCIRPTION AND PRESLECTION . . . . . . 78

5.1 BABAR experimental data $\ldots \ldots \ldots \ldots . \ldots 78$

5.2 Monte Carlo simulation . . . . . . . . . . . . . . . . . . . . . . . 79

5.2 .1 Generic Monte Carlo . . . . . . . . . . . . . . . . 80

5.2 .2 Exclusive Monte Carlo production . . . . . . . . . . 81

5.3 Event Preselection . . . . . . . . . . . . . . . . . . . . . . 82

5.3 .1 Skim . . . . . . . . . . . . . . . . . . . . 83

5.3 .2 Reskimming . . . . . . . . . . . . . . . . . 84

6 EVENT SELECTION . . . . . . . . . . . . . . . . . . . 89

6.1 Event Reconstruction and Selection _. . . . . . . . . . . 89

6.1.1 Long-lived $K_{S}^{0}, \Lambda$ and $\Xi^{-}$reconstruction and selection . . 89

$6.1 .2 \Xi_{c}^{0}$ reconstruction and selection . . . . . . . . . . 93

$6.1 .3 \Xi_{c}^{+}$Selection . . . . . . . . . . . . . . . 96

6.1.4 Reconstruction and selection of $\Lambda_{c}^{+} \ldots \ldots \ldots \ldots$. . . 97

$6.2 B$ meson reconstruction and selection $\ldots \ldots \ldots \ldots$

$6.2 .1 m_{\mathrm{ES}}$ and $\Delta E$ variables $\ldots \ldots \ldots \ldots \ldots \ldots \ldots \ldots$

6.2 .2 Signal shape in Monte Carlo . . . . . . . . . . . . . . . . 105

6.2.3 Choice of best candidate selection . . . . . . . . . . 108

6.2.4 A summary of the selection of $B$ meson decays . . . . . 111

$7 \quad B$ MESON SIGNAL EXTRACTION $\ldots \ldots \ldots \ldots \ldots \ldots$

7.1 Efficiency study . . . . . . . . . . . . . . . . . . . . . . . 114

7.1.1 What is a signal event in the MC? . . . . . . . . . 114 
7.1.2 Effect of final state radiation (FSR) on efficiency . . . . . 116

7.1.3 Tracking efficiency correction . . . . . . . . . . . . 119

7.1.4 Effect of intermediate resonance on efficiency . . . . . . . 119

7.1.5 The crosscheck of efficiencies of three-body $B$ decays . . . 120

7.2 The maximum likelihood fit and and fit validation . . . . . . . . 121

7.2.1 Unbinned maximum likelihood fit . . . . . . . . . . . 121

7.2.2 The modeling of the PDF . . . . . . . . . . . . . . 122

7.2.3 Fit strategy and result . . . . . . . . . . . . . . . 123

7.3 Background Study . . . . . . . . . . . . . . . . . . . 127

7.3.1 Background study of the $B \rightarrow \Xi_{c} \bar{\Lambda}_{c}^{-}$decay modes . . . . 127

7.3.2 Background study for $B^{-} \rightarrow \Lambda_{c}^{+} \bar{\Lambda}_{c}^{-} K^{-}$Decays . . . . . . 134

7.4 Estimation of the systematic uncertainties . . . . . . . . . . . . . 144

7.4.1 B counting systematics . . . . . . . . . . . . . . . 144

7.4.2 Tracking efficiency . . . . . . . . . . . . . . . . . 145

7.4.3 Particle identification . . . . . . . . . . . . . . 145

7.4.4 Monte Carlo statistics . . . . . . . . . . . . . . . 147

7.4.5 Effect of intermediate resonances in $\Lambda_{c}^{+}$decays . . . . . . 147

7.4.6 Uncertainties in the decay branching fractions . . . . . . 147

7.4.7 The difference in $\Delta E$ between data and MC . . . . . . . 148

7.4.8 Maximum likelihood fits and signal parameterizations . . 148

7.4.9 Summary of systematics uncertainties . . . . . . . . . . 148

8 RESULTS AND DISCUSSION . . . . . . . . . . . . . . . . . . . 152

8.1 Branching fraction calculation . . . . . . . . . . . . . . 152

$8.1 .1 \quad \bar{B} \rightarrow \Xi_{c} \bar{\Lambda}_{c}^{-}$decays . . . . . . . . . . . . . . 152

$8.1 .2 \quad \bar{B} \rightarrow \Lambda_{c}^{+} \bar{\Lambda}_{c}^{-} \bar{K}$ decays . . . . . . . . . . . . . 153

8.1.3 Combining the branching fractions . . . . . . . . . . . 154

8.2 Discussion of the results . . . . . . . . . . . . . . . 159

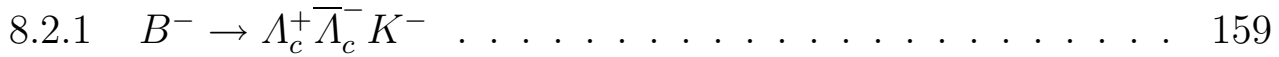

$8.2 .2 \quad B^{-} \rightarrow \Xi_{c}^{0} \bar{\Lambda}_{c}^{-} \ldots \ldots \ldots . \ldots . \ldots . . \ldots 160$

8.3 Study of intermediate resonances in $B^{-} \rightarrow \Lambda_{c}^{+} \bar{\Lambda}_{c}^{-} K^{-}$decay $\ldots 162$

9 SUMMARY AND OUTLOOK . . . . . . . . . . . . . . 165

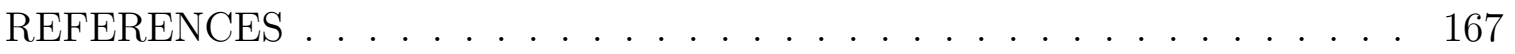




\section{LIST OF TABLES}

Table

1.1 Properties of fermions in the Standard Model. . . . . . . . . . . . . . 2

1.2 Properties of gauge Bosons in the Standard Model . . . . . . . . . . . . . 6

1.3 Properties of the $\mathrm{L}=0$ charmed baryons . . . . . . . . . . . . . . . 15

1.4 Branching ratio of inclusive $B$ decays to baryons (PDG) . . . . . . . . 21

1.5 Bell results on exclusive $B$ decays to charmed baryon pairs . . . . . . . 24

1.6 List of decay channels studied _. . . . . . . . . . . . . . . . 27

2.1 Cross-sections, production and trigger rates for the principle processes at $\sqrt{s}=M(\Upsilon(4 S)) \ldots \ldots \ldots \ldots$. . . . . . . . . . . . . . . . .

3.1 Trigger primitives for the DCT and EMT . . . . . . . . . . 60

3.2 IFT trigger primitive . . . . . . . . . . . . . . . . 61

4.1 Criteria used for selecting available categories of hadron candidates . . . 75

5.1 BABAR integrated luminosity and number of $B \bar{B}$ pairs. . . . . . . . . . 78

5.2 Generic Monte Carlo samples. . . . . . . . . . . . . . . . . . . 81

5.3 Signal Monte Carlo samples. . . . . . . . . . . . . . . . . . 87

5.4 Summary of private $\mathrm{MC} \ldots \ldots \ldots$. . . . . . . . . . . 88

6.1 The branching fractions of the $\Lambda_{c}^{+}$decay modes $[\mathrm{PDG} 2006] \ldots 98$

$6.2 m_{\mathrm{ES}}$ and $\Delta E$ resolution $\ldots \ldots \ldots \ldots \ldots$

7.1 Overall efficiency from fitting signal MC . . . . . . . . . . . . . 116

7.2 Number of photons in the FSR events . . . . . . . . . . . . . 117

7.3 Summary of efficiencies for various assumptions about intermediate resonances in $\Lambda_{c}^{+} \rightarrow p^{+} K^{-} \pi^{+}$decays, measured from 4 signal MC samples . . 120 
7.4 Fitted yields from the Run 1-4 on-peak data and likelihood difference $\Delta \log \mathcal{L}$ between the fits with signal plus background and with background only . . . . . . . . . . . . . . . . . . . . 126

7.5 The remaining events in the $B$ signal region for each of the five modes reconstructed from each of the five signal MC dataset after all the selection cuts are applied to each signal modes respectively . . . . . . . . . . . . 138

7.6 Summary of correction factors and uncertainties on the efficiency from tracking . . . . . . . . . . . . . . 146

7.7 Systematic uncertainty due to the PID . . . . . . . . . . . 147

7.8 Summary of uncertainties from the known decay branching fractions(\%) and corrections $(\%) \ldots \ldots \ldots \ldots \ldots \ldots$

7.9 Summary of the relative systematic uncertainties(\%) of $B^{-} \rightarrow \Lambda_{c}^{+} \bar{\Lambda}_{c}^{-} K^{-}$

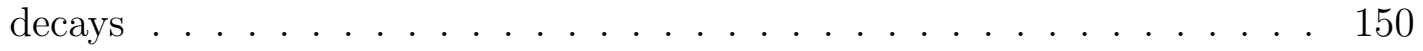

7.10 Summary of the relative systematic uncertainties(\%) of $B^{-} \rightarrow \Xi_{c}^{0} \bar{\Lambda}_{c}^{-}$decays 150

7.11 Summary of the relative systematic uncertainties(\%) of $\bar{B}^{0} \rightarrow \Xi_{c}^{+} \bar{\Lambda}_{c}^{-}$and

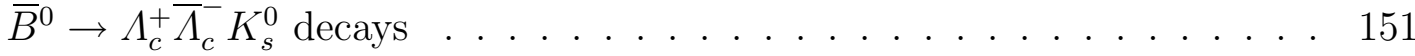

8.1 The summary of the relative systematic uncertainties(\%) of $B^{-} \rightarrow \Lambda_{c}^{+} \bar{\Lambda}_{c}^{-} K^{-}$ and $B^{-} \rightarrow \Xi_{c}^{0} \bar{\Lambda}_{c}^{-}$decays $\ldots \ldots \ldots \ldots \ldots \ldots \ldots \ldots \ldots$

8.2 Summary of luminosities, $B \bar{B}$ pairs, the fitted signal yields, the individual and overall efficiencies (\%) and the branching fractions. . . . . . . 160

8.3 Summary of luminosities, $B \bar{B}$ pairs, the fitted signal yields, the overall efficiencies and the products of branching fractions . . . . . . . . 161 


\section{LIST OF FIGURES}

Figure

1.1 The unitary triangle with the definition of $\rho, \eta$, and CKM angles $\alpha, \beta$ and $\gamma \ldots \ldots \ldots \ldots \ldots \ldots \ldots \ldots \ldots \ldots \ldots \ldots \ldots \ldots \ldots \ldots \ldots$

1.2 $\mathrm{SU}(4)$ baryon multiplets which show all the $\mathrm{L}=0$ baryons in a four-quark system $\ldots \ldots \ldots$

1.3 Tree level Feynman diagram illustrating the decay mechanism of $\Xi_{c}^{0} \rightarrow$

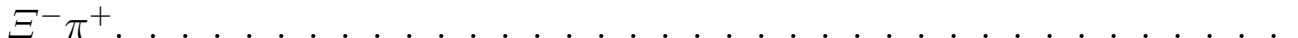

1.4 Mass spectrum for known charmed baryon. Labels along the x-axis show the quark content of charmed baryons (no s quark, one s quark, two s quarks from left to right. The $J^{P}$ 's are theoretical prediction, and no measurement exists.

1.5 Quark level Feynman Diagram for the processes $\bar{B} \rightarrow \Lambda_{c}^{+} \bar{\Lambda}_{c}^{-} \bar{K}$ and $\bar{B} \rightarrow$ $\Xi_{c} \bar{\Lambda}_{c}^{-}$. When $q=d, \bar{B}$ represents $\bar{B}^{0}$, and when $q=u, \bar{B}$ represents $B^{-}$. $q^{\prime}$ can be either $u$ or $d$ quark. . . . . . . . . . . . . . . . . . . .

1.6 The flavor diagram of $B^{-} \rightarrow \Lambda_{c}^{+} \bar{\Lambda}_{c}^{-} K^{-}$which is arisen via $B^{0} \rightarrow D^{0} D^{0} K^{-}$

2.1 The schematic view of PEP-II.

2.2 The cross-section of $e^{+} e^{-} \rightarrow$ hadrons at the $\Upsilon$ mesons $(1 \mathrm{~S}-4 \mathrm{~S})$ and in the continuum measured by the CLEO collaboration $[36] . . . .$.

2.3 The schematic of interaction region. . . . . . . . . . . . . .

2.4 The longitudinal section of the detector. The dimension is in mm. . . . . 34

2.5 The transverse section of SVT. . . . . . . . . . . . . . . . . 36

2.6 The longitudinal section of SVT. The roman numbers label the six different types of sensors. . . . . . . . . . . . . . . . .

2.7 The side view of drift chamber. The dimension is in $\mathrm{mm}$. The interaction point is offset from the center of DCH. . . . . . . . . . . . . . . 
2.8 The transverse view of the drift chamber. (a) The transverse view of the wires in the drift chamber. (b) Schematic layout of the drift cell in the first four superlayers. The dimension of the numbers on the right side in mrad. . . . . . . . . . . . . . . . . . . 40

2.9 The differences of four tracking parameters. . . . . . . . . . . . . . 41

2.10 The measured $d E / d x$ in the drift chamber as a function of track momentum with superimposed curves derived from the Bethe-Bloch prediction.

2.11 A view of the DIRC system. . . . . . . . . . . . . . . . 44

2.12 The elevated view of the nominal DIRC system geometry. All the dimensions are in mm. . . . . . . . . . . . . . . . . . . 45

2.13 A view of the DIRC system. . . . . . . . . . . . . . 45

2.14 The Cherenkov angle of tracks distribution plotted according the momentum of the tracks. The grey lines are the predicted values. . . . . . . . . 46

2.15 The longitudinal view of the top half of the EMC. All the dimensions are in mm. . . . . . . . . . . . . . . . . . 48

2.16 IFR structure: barrel sector, forward endcap and backward endcap. . . . 49

2.17 IFR structure: barrel sector, forward endcap and backward endcap. . . . 50

3.1 The BABAR electronics, trigger, DAQ system . . . . . . . . . . . . . 52

$3.2 \mathrm{X}$-Y view of the drift chamber, illustrating definition of terms. . . . . . . 55

3.3 The represent of TSF pivot group. Cell 4 is called pivot cell, and cell 0-7 form a pivot group. . . . . . . . . . . . . . . . 56

3.4 The DCT track efficiency versus momentum for $\mathrm{A}, \mathrm{B}$, and $A^{\prime}$ tracks. . . 58

3.5 The block diagram of the global Level 1 Trigger module. . . . . . . . . . 62

3.6 Single track z0 for tracks in all L1 triggered events, reconstructed by L3. 65

3.7 The updated trigger system. . . . . . . . . . . . . . . 66

3.8 ZPD block diagram. . . . . . . . . . . . . . . . . . 69 
3.9 The left plot is ZPD tracking efficiency, defined as the fraction of tracks offline reconstructed as coming from the IP which pass the L1 trigger selection criteria. Upper (red) is isolated tracks, and lower (blue) is the tracks in the hadronic events. The right plot is $\mathrm{ZPD} z_{0}$ resolution, defined as the difference between ZPD reconstructed and offline reconstructed tracks. .

4.1 A possible decay tree for $B^{-} \rightarrow \Xi_{c}^{0} \bar{\Lambda}_{c}^{-} \quad \ldots \ldots \ldots \ldots$. . . . . . 77

6.1 Invariant mass spectra of the intermediate hyperon from representative subsamples in data. The left plot shows the invariant mass distribution of $\Lambda^{0}\left(p K \pi^{+}\right)$from $15 \mathrm{fb}^{-1}$ data; and the plot on the right side shows the invariant mass distribution of $\Xi^{-}\left(\Lambda^{0} \pi^{-}\right)$from $99 \mathrm{fb}^{-1}$ data. . . . . . .

6.2 The variation of $\Xi^{-}$raw yield (left) and number of DCH hits (right) over the time periods. . . . . . . . . . . . . . .

6.3 Momentum distribution of $K_{S}^{0}$ in the signal Monte Carlo, red line represents the momentum distribution of the $K_{S}^{0}$ decayed from $B$, black line represents the momentum distribution of the $K_{S}^{0}$ decayed from $\Lambda_{c}^{+}$. . .

6.4 Invariant Mass distribution of $\Xi_{c}^{0} \rightarrow \Xi^{-} \pi^{+}$candidates from the BABAR data (left), and from truth-matched signal MC (right). On the left, the signal is fitted with a single Gaussian, where the fitted mean is $2471.15 \pm$ $0.18 \mathrm{MeV}$ with $\sigma=6.07 \pm 0.18 \mathrm{MeV}$. On the right, the signal is fitted with a double-Gaussian; the fitted parameters are mean $=2472.16 \pm 0.13 \mathrm{MeV}$, core Gaussian $\sigma_{1}=5.79 \pm 0.11 \mathrm{MeV}$ with $92.2 \pm 0.9 \%$ of the yield, and $\sigma_{2}=29.17 \pm 2.08 \mathrm{MeV} \ldots \ldots \ldots \ldots \ldots \ldots \ldots$

6.5 $p^{*}$ distribution from reconstructed $\Xi_{c}^{0}$ in on-peak data. The distribution for the $\Xi_{c}^{0}$ signal region (see text). is plotted as black points in (a) for the $\Xi^{-} \pi^{+}$mode. The $p^{*}$ distribution in sidebands is also shown as green/light points. and are fitted to a smooth curve, superimposed on the data. The sideband-subtracted $p^{*}$ distributions are shown in (b). The lower peak below $p^{*}<1.5 \mathrm{GeV} / \mathrm{c}$ is primarily from the $\Xi_{c}^{0}$ production from $\mathrm{B} / \overline{\mathrm{B}}$

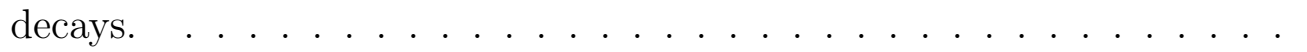

6.6 Invariant mass distribution of $\Xi_{c}^{0} \rightarrow \Lambda^{0} K^{-} \pi^{+}$candidates from the BABAR data (left), and from truth-matched signal MC (right). On the left, the signal is fitted with a single Gaussian; the fitted mean is $2470.4 \pm 0.3 \mathrm{MeV}$ with $\sigma=4.93 \pm 0.29 \mathrm{MeV}$. On the right, the signal is fitted with a doubleGaussian; the fitted mean $=2471.41 \pm 0.06 \mathrm{MeV}$, core Gaussian $\sigma_{1}=4.62 \pm$ $0.06 \mathrm{MeV}$ with $82.6 \pm 0.6 \%$ of the yield, and $\sigma_{2}=25.35 \pm 0.55 \mathrm{MeV}$. . . 
6.7 Invariant mass distribution of the $\Xi_{c}^{0} \rightarrow \Lambda^{0} K^{-} \pi^{+}$in the BABAR data, where a center of mass momentum $p^{*}>2.2 \mathrm{GeV}$ cut is made. . . . . . .

6.8 Invariant mass distribution of $\Xi_{c}^{0} \rightarrow \Sigma^{0} K^{-} \pi^{+}$candidate in the $c \bar{c}$ generic $\mathrm{MC}$ (a) and the BABAR off-peak data (b). (a), the invariant mass of $\Lambda^{0} \gamma K^{-} \pi^{-}$is shown as black line with fit curve, the invariant mass of $\Lambda^{0} K^{-} \pi^{-}$is shown as blue line, truth matched $\Xi_{c}^{0}$ are shown as red filled area, and truth matched $\Xi_{c}^{0}$ with the $\gamma$ dropped are shown as pink hatched area); (b) the invariant mass of $\Lambda^{0} \gamma K^{-} \pi^{-}$is shown as black line, the invariant mass of $\Lambda^{0} K^{-} \pi^{-}$is shown as red line. The blue solid histogram shows the $\left(\Lambda K^{-} \pi^{+}\right)$invariant mass for candidates with $\left(\Lambda \gamma K^{-} \pi^{+}\right)$invariant mass in the range of $(2.47,2.5) \mathrm{GeV} / c^{2}$. . . . . . . . . .

6.9 Invariant mass distribution of $\Xi_{c}^{+} \rightarrow \Xi^{-} \pi^{+} \pi^{+}$candidates from the BABAR data on the left, and from truth-matched signal MC on the right. On the left, the signal is fitted with a single Gaussian; the fitted mean is $2467.68 \pm 0.14 \mathrm{MeV}$ with $\sigma=5.68 \pm 0.15 \mathrm{MeV}$. On the right, the signal is fitted with a triple-Gaussian;the fitted parameters are: mean $=2467.15 \pm$ $0.04 \mathrm{GeV}$, core Gaussian $\sigma_{1}=4.49 \pm 0.08 \mathrm{MeV}$ with $68.09 \pm 1.89 \%$ of the yield, $\sigma_{2}=11.39 \pm 0.49 \mathrm{MeV}$ with $25.37 \pm 1.67$ of the yield, and $\sigma_{3}=39.54 \pm 0.16 \mathrm{MeV} \ldots \ldots \ldots \ldots \ldots \ldots \ldots$

6.10 Invariant mass distribution of $\Lambda_{c}^{+} \rightarrow p^{+} K^{-} \pi^{+}$candidates in the BABAR data (left) and in truth-matched signal Monte Carlo (right). On the left, the signal is fitted to a double-Gaussian with common mean. The Gaussian mean is $2285.88 \pm 0.06 \mathrm{MeV}, \sigma_{1}=4.0 \pm 0.2 \mathrm{MeV}$ with a yield fraction of $62.3 \%$, and $\sigma_{2}$ is $8.86 \pm 0.96 \mathrm{MeV}$. On the right, the signal is fitted to a triple-Gaussian, with mean $2285.11 \pm 0.03 \mathrm{MeV}, \sigma_{1}=3.80 \pm 0.07 \mathrm{MeV}$ with a yield of fraction of $66 \%, \sigma_{2}=9.22 \pm 0.85 \mathrm{MeV}$ with a yield fraction of $20 \%$ and $\sigma_{3}=24.65 \pm 1.47 \mathrm{MeV} \ldots \ldots \ldots \ldots \ldots$

6.11 Invariant mass distribution of $\Lambda_{c}^{+} \rightarrow p^{+} K_{S}^{0}$ candidates in the BABAR data (left) and in truth-matched signal Monte Carlo (right). On the left, the signal is fitted to a single Gaussian on linear background. The Gaussian mean is $2287.02 \pm 0.12 \mathrm{MeV}$ with $\sigma=5.83 \pm 0.13 \mathrm{MeV}$. On the right, the signal is fitted to a triple-Gaussian, with mean $2286.41 \pm 0.04, \sigma_{1}=$ $4.85 \pm 0.14 \mathrm{MeV}$ with a yield fraction of $67 \%, \sigma_{2}=25.59 \pm 1.46 \mathrm{MeV}$ with a yield fraction of $12 \%$ and $\sigma_{3}=8.63 \pm 0.98 \mathrm{MeV} \ldots \ldots \ldots \ldots$

6.12 Invariant mass distribution of $\Lambda_{c}^{+} \rightarrow \Lambda^{0} \pi^{+}$from $18 \mathrm{fb}^{-1}$ of data: (a) $p^{*}<$ $2 \mathrm{GeV}$, and (b) $p^{*}>2 \mathrm{GeV} \ldots \ldots \ldots \ldots \ldots$ 
6.13 Invariant mass distribution of $\Lambda_{c}^{+} \rightarrow \Lambda^{0} \pi^{+}$in the $B A B A R$ data (left) and in truth-matched Monte Carlo (right). On the left, the signal is fitted to a single Gaussian on a linear background. The fitted mean is $2287.13 \pm$ $0.19 \mathrm{MeV}$ with $\sigma=5.26 \pm 0.20 \mathrm{MeV}$. On the right, the signal is fitted to a triple-Gaussian, with mean $=2286.53 \pm 0.04 \mathrm{MeV}, \sigma_{1}=4.63 \pm 0.35 \mathrm{MeV}$ with a yield fraction of $52 \%, \sigma_{2}=7.06 \pm 0.66 \mathrm{MeV}$ with a yield fraction of $40 \%$ and $\sigma_{3}=29.37 \pm 2.39 \mathrm{MeV} \ldots \ldots \ldots \ldots$

6.14 The $m_{\mathrm{ES}}-\Delta E$ region used to define signal and background regions. . . .

$6.15 \Delta E$ vs. $m_{\mathrm{ES}}$ for a signal $\mathrm{MC}$ sample of $B^{-} \rightarrow \Lambda_{c}^{+} \bar{\Lambda}_{c}^{-} K^{-}$, and both $\Lambda_{c}^{+}$ decay to $p K \pi$ unconstrained (left) and constrained (right) $\Lambda_{c}^{+}$candidates masses, passed all the selection criteria. . . . . . . . . . . . . .

$6.16 m_{\mathrm{ES}}$ for a signal MC sample of $B^{-} \rightarrow \Lambda_{c}^{+} \bar{\Lambda}_{c}^{-} K^{-}$, and both $\Lambda_{c}^{+}$decay to $p K \pi$ unconstrained (left) and constrained (right) $\Lambda_{c}^{+}$candidates masses, passed all the selection criteria.

$6.17 \Delta E$ for a signal MC sample of $B^{-} \rightarrow \Lambda_{c}^{+} \bar{\Lambda}_{c}^{-} K^{-}$, and both $\Lambda_{c}^{+}$decay to $p K \pi$ unconstrained (left) and constrained (right) $\Lambda_{c}^{+}$candidates masses, passed all the selection criteria. Truth matching are applied here. . . . . 108

$6.18 \Delta E$ vs. $m_{\mathrm{ES}}$ distribution of $B^{-} \rightarrow \Xi_{c}^{0} \bar{\Lambda}_{c}^{-}$from on-peak dataset: (a) $\Xi_{c}^{0} \rightarrow \Xi^{-} \pi^{+}$; (b) $\Xi_{c}^{0} \rightarrow \Lambda^{0} K^{-} \pi^{+} \ldots \ldots \ldots$. . . . . . . . .

$6.19 \Delta E$ vs. $m_{\mathrm{ES}}$ distribution of $B^{-} \rightarrow \Lambda_{c}^{+} \bar{\Lambda}_{c}^{-} K^{-}$from on-peak dataset: (a) $\Lambda_{c}^{+} \rightarrow p^{+} K^{-} \pi^{+}, \bar{\Lambda}_{c}^{-} \rightarrow p^{-} K^{+} \pi^{-}$, (b) $\Lambda_{c}^{+} \rightarrow p^{+} K^{-} \pi^{+}, \bar{\Lambda}_{c}^{-} \rightarrow p^{-} K_{S}^{0}$, (c) $\Lambda_{c}^{+} \rightarrow p^{+} K_{S}^{0}, \bar{\Lambda}_{c}^{-} \rightarrow p^{-} K^{+} \pi^{-}(\mathrm{d}) \Lambda_{c}^{+} \rightarrow p^{+} K^{-} \pi^{+}, \bar{\Lambda}_{c}^{-} \rightarrow \bar{\Lambda}^{0} \pi^{-}$(e) $\Lambda_{c}^{+} \rightarrow$ $\Lambda^{0} \pi^{+}, \bar{\Lambda}_{c}^{-} \rightarrow p^{-} K^{+} \pi^{-} \ldots \ldots \ldots \ldots \ldots \ldots$

7.1 The energy of FSR photon in the signal $\mathrm{MC}$ for $B^{-} \rightarrow \Lambda_{c}^{+} \bar{\Lambda}_{c}^{-} K^{-}, \Lambda_{c}^{+} \rightarrow$ $p^{+} K^{-} \pi^{+}$. (a)B daughter, (b)the daughter gamma of first $\Lambda_{c}^{+}$, (c) the daughter gamma of second daughter.

7.2 The $\Delta E$ distribution of the $B$ candidates $\left(B^{-} \rightarrow \Lambda_{c}^{+} \bar{\Lambda}_{c}^{-} K^{-}, \Lambda_{c}^{+} \rightarrow p^{+} K^{-} \pi^{+}\right)$ with FSR photons in the signal MC after all selection cuts, except $\Delta E$, including the best candidate selection. . . . . . . . . . . . . 118

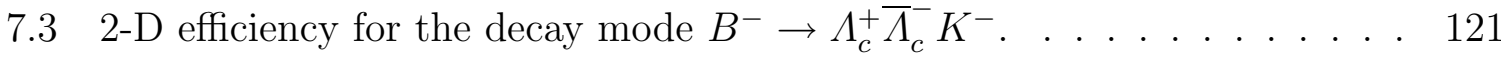


$7.4 m_{\mathrm{ES}}$ fit of $B^{-} \rightarrow \Lambda_{c}^{+} \bar{\Lambda}_{c}^{-} K^{-}$to the Run 1-4 on-peak data. (a) $\Lambda_{c}^{+} \rightarrow$ $p^{+} K^{-} \pi^{+}, \bar{\Lambda}_{c}^{-} \rightarrow p^{-} K^{+} \pi^{-}$; (b) $\Lambda_{c}^{+} \rightarrow p^{+} K^{-} \pi^{+}, \bar{\Lambda}_{c}^{-} \rightarrow p^{-} K_{s}^{0}$; (c) $\Lambda_{c}^{+} \rightarrow$ $p^{+} K^{-} \pi^{+}, \bar{\Lambda}_{c}^{-} \rightarrow \bar{\Lambda}^{0} \pi^{-}$; (d) $\Lambda_{c}^{+} \rightarrow p^{+} K_{S}^{0}, \bar{\Lambda}_{c}^{-} \rightarrow p^{-} K^{+} \pi^{-}$; (e) $\Lambda_{c}^{+} \rightarrow \Lambda^{0} \pi^{+}$, $\bar{\Lambda}_{c}^{-} \rightarrow p^{-} K^{+} \pi^{-} \ldots \ldots \ldots \ldots \ldots \ldots \ldots \ldots$

7.5 $m_{\mathrm{ES}}$ fit of $B^{-} \rightarrow \Lambda_{c}^{+} \bar{\Lambda}_{c}^{-} K^{-}$to the Run 1-4 on-peak data in all five modes. 125

7.6 $m_{\mathrm{ES}}$ fit of $B^{-} \rightarrow \Xi_{c}^{0} \bar{\Lambda}_{c}^{-}$: (a) $\Xi_{c}^{0} \rightarrow \Xi^{-} \pi^{+}$, and (b) $\Xi_{c}^{0} \rightarrow \Lambda^{0} K^{-} \pi^{+}$(right) .

$7.7 m_{\mathrm{ES}}$ fit of (a) $\bar{B}^{0} \rightarrow \Xi_{c}^{+} \bar{\Lambda}_{c}^{-}$, (b) $\bar{B}^{0} \rightarrow \Lambda_{c}^{+} \bar{\Lambda}_{c}^{-} K_{s}^{0} \ldots \ldots \ldots$

7.8 The distribution of $m_{\mathrm{ES}}$ vs. $\Delta E$ for the 4 example feed-down modes in which a pion, two pions or a $\gamma$ not included. . . . . . . . . . . . . . .

7.9 $\Delta E$ distribution of $B^{-} \rightarrow \Xi_{c}^{0} \bar{\Lambda}_{c}^{-}$from the $B^{-} \rightarrow \Xi_{c}^{0} \bar{\Lambda}_{c}^{-}$signal $\mathrm{MC}$ and cross-feed mode(normalized distribution). The black histogram shows the signal mode $B^{-} \rightarrow \Xi_{c}^{0} \bar{\Lambda}_{c}^{-}$. The colored histograms show contributions from example feed-down modes: $B^{-} \rightarrow \Xi_{c}^{\prime} 0 \bar{\Lambda}_{c}^{-}$(red), $\bar{B}^{0} \rightarrow \Xi_{c}^{0} \bar{\Lambda}_{c}^{-} \pi^{+}$ (green), $B^{-} \rightarrow \Xi_{c}^{0} \bar{\Lambda}_{c}^{-} \pi^{0}$ (blue), $B^{-} \rightarrow \Xi_{c}^{0} \bar{\Sigma}_{c}^{-} \pi^{0}$ (yellow). . . . . . 130

7.10 The $m_{\mathrm{ES}}$ and $\Delta E$ distributions of $B^{-} \rightarrow \Xi_{c}^{0} \bar{\Lambda}_{c}^{-}$reconstructed from Generic $B^{+} B^{-}$MC. (a) $\Delta E$ distribution with $m_{\mathrm{ES}}>5 / 27 \mathrm{GeV}$, (b) 2 d plot of $\Delta E$ vs. $m_{\mathrm{ES}}$ (c) $m_{\mathrm{ES}}$ distribution with $|\Delta E|<0.03 \mathrm{GeV} \ldots \ldots \ldots$

$7.11 m_{\mathrm{ES}}$ distribution from generic $B^{+} \bar{B}^{-} \ldots \ldots \ldots \ldots \ldots$

$7.12 m_{\mathrm{ES}}$ distribution of the $B$ candidate $\left(\Xi_{c}^{0} \bar{\Lambda}_{c}^{-}\right)$with different cut on the invariant mass of $p K \pi$ from the run 1-4 data. (a) $\left|m_{p K \pi}-\bar{m}\right|<2 \sigma$; (b) $\left|m_{p K \pi}-\bar{m}\right|<3 \sigma ;$ (c) $\left|m_{p K \pi}-\bar{m}\right|<6 \sigma$; (d) $\left|m_{p K \pi}-\bar{m}\right|<9 \sigma \ldots$. .

7.13 Mass distribution $\Lambda_{c}^{+}$and $\Xi_{c}$, the daughters of the $\bar{B}^{0} \rightarrow \Xi_{c}^{+} \bar{\Lambda}_{c}^{-}$reconstructed from MC dataset of $B^{0} \rightarrow \bar{\Lambda}_{c}^{-} p^{+} \pi^{-} \pi^{+}$and $\bar{B}^{0} \rightarrow \Xi_{c}^{+} \bar{\Lambda}^{0} \pi^{-} \ldots$

$7.14 m_{\mathrm{ES}}$ and $\Delta E$ distribution of the $\bar{B}^{0} \rightarrow \Xi_{c}^{+} \bar{\Lambda}_{c}^{-}$reconstructed from MC dataset of $B^{0} \rightarrow \bar{\Lambda}_{c}^{-} p^{+} \pi^{-} \pi^{+}$and $\bar{B}^{0} \rightarrow \Xi_{c}^{+} \bar{\Lambda}^{0} \pi^{-}$. 
7.15 The $m_{\mathrm{ES}}$ distribution from a sample of simulated $\bar{B}^{0} \rightarrow \Lambda_{c}^{+} \bar{\Lambda}_{c}^{-} K_{s}^{0}$ events, reconstructed as $B^{-} \rightarrow \Lambda_{c}^{+} \bar{\Lambda}_{c}^{-} K^{-}$and passing all selection criteria. The same data are shown in both plots. In the left plot, the signal mean and width parameters are floated, whereas in the right plot, they are fixed to the values obtained for $B^{-} \rightarrow \Lambda_{c}^{+} \bar{\Lambda}_{c}^{-} K^{-}$in data $\left(m_{0}=5.2796 \mathrm{GeV}\right.$ and $\sigma=2.62 \mathrm{MeV}) . \ldots \ldots \ldots \ldots \ldots$

$7.16 m_{\mathrm{ES}}$ distributions of cross-feed background from the following MC samples: (a) mis-reconstructed $B^{-} \rightarrow \Lambda_{c}^{+}\left(p K_{S}^{0}\right) \bar{\Lambda}_{c}^{-}(p K \pi) K^{-}$candidates from the $B^{-} \rightarrow \Lambda_{c}^{+}(p K \pi) \bar{\Lambda}_{c}^{-}\left(p K_{S}^{0}\right) K^{-}$signal MC dataset, (b) mis-reconstructed $B^{-} \rightarrow \Lambda_{c}^{+}(p K \pi) \bar{\Lambda}_{c}^{-}\left(p K_{S}^{0}\right) K^{-}$candidates from the $B^{-} \rightarrow \Lambda_{c}^{+}\left(p K_{S}^{0}\right) \bar{\Lambda}_{c}^{-}(p K \pi) K^{-}$ signal MC dataset, (c) mis-reconstructed $B^{-} \rightarrow \Lambda_{c}^{+}\left(\Lambda^{0} \pi\right) \bar{\Lambda}_{c}^{-}(p K \pi) K^{-}$ candidates from the $B^{-} \rightarrow \Lambda_{c}^{+}(p K \pi) \bar{\Lambda}_{c}^{-}\left(\bar{\Lambda}^{0} \pi\right) K^{-}$signal MC datset, (d) mis-reconstructed $B^{-} \rightarrow \Lambda_{c}^{+}(p K \pi) \bar{\Lambda}_{c}^{-}\left(\bar{\Lambda}^{0} \pi\right) K^{-}$candidates from the $B^{-} \rightarrow$ $\Lambda_{c}^{+}\left(\Lambda^{0} \pi\right) \bar{\Lambda}_{c}^{-}(p K \pi) K^{-}$signal MC datset. For each of the $m_{\mathrm{ES}}$ distribution, we fit it to Gaussian plus Argus function. . . . . . . . . . . . . . .

7.17 Distribution of $m_{\mathrm{ES}}$ and $\Delta E$ of $B^{-} \rightarrow \Lambda_{c}^{+} \bar{\Lambda}_{c}^{-} K^{-}\left(\Lambda_{c}^{+} \rightarrow p^{+} K^{-} \pi^{+}\right.$for both $\Lambda_{c}^{+}$) candidates for data (black point) and Generic $B \bar{B}+q \bar{q} \mathrm{MC}$ (shaded area) samples. The generic $B^{0} \bar{B}^{0}, B^{+} B^{-}, c \bar{c}$ and $u d s$ MC samples are individually scaled to have luminosities equal to the data sample. (a) $m_{\mathrm{ES}}$ distribution with $|\Delta E|<0.2 \mathrm{GeV}$ (b) $\Delta E$ distribution with $m_{\mathrm{ES}}>5.20 \mathrm{GeV} 141$

7.18 Mass distribution of (a) the first $\Lambda_{c}^{+}$and (b) the second $\Lambda_{c}^{+}$of $B^{-} \rightarrow$ $\Lambda_{c}^{+} \bar{\Lambda}_{c}^{-} K^{-}\left(\Lambda_{c}^{+} \rightarrow p^{+} K^{-} \pi^{+}\right.$for both $\left.\Lambda_{c}^{+}\right)$for data and Generic MC samples where we require $m_{\mathrm{ES}}>5.2 \mathrm{GeV}$ and $|\Delta E|<0.2 \mathrm{GeV}$ and we make $3 \sigma_{m}$ mass cut for the other $\Lambda_{c}^{+}$when we plot one $\Lambda_{c}^{+}$mass distribution. The generic $B^{0} \bar{B}^{0}, B^{+} B^{-}, c \bar{c}$ and $u d s$ MC samples are individually scaled to have luminosities equal to the data sample.

$7.19 m_{\mathrm{ES}}$ distribution of $B^{-} \rightarrow \Lambda_{c}^{+} \bar{\Lambda}_{c}^{-} K^{-}$(both $\Lambda_{c}^{+} \rightarrow p^{+} K^{-} \pi^{+}$) candidate fitted with an Argus function, In (a), events from the combined $B^{0} \bar{B}^{0}$ and $B^{+} B^{-}$generic $\mathrm{MC}$ samples are shown with a cut of $|\Delta E|<0.03$. In (b), events from the $\Delta E$ sideband of data are shown $\left(m_{E S}>5.2 \mathrm{GeV}\right.$ and $|\Delta E|>0.05 \mathrm{GeV}$ ). The normalization in (a) is not scale to match the data integrated luminosity. . . . . . . . . . . . . . . . .

$7.20 p^{*}$ distribution of (a) the first $\Lambda_{c}^{+}$and (b) the second $\Lambda_{c}^{+}$of $B^{-} \rightarrow \Lambda_{c}^{+} \bar{\Lambda}_{c}^{-} K^{-}$ $\left(\Lambda_{c}^{+} \rightarrow p^{+} K^{-} \pi^{+}\right.$for both $\left.\Lambda_{c}^{+}\right)$for data and Generic MC samples where the $m_{\mathrm{ES}}>5.20 \mathrm{GeV}$ and $|\Delta E|<0.2 \mathrm{GeV}$. The generic $B^{0} \bar{B}^{0}, B^{+} B^{-}, c \bar{c}$ and $u d s \mathrm{MC}$ samples are individually scaled to have luminosities equal to the data sample. 
7.21 Distribution of (a) thrust (b) $\cos ^{*} \Theta_{B}$ and (c) R2 of $B^{-} \rightarrow \Lambda_{c}^{+} \bar{\Lambda}_{c}^{-} K^{-}$ $\left(\Lambda_{c}^{+} \rightarrow p^{+} K^{-} \pi^{+}\right.$for both $\left.\Lambda_{c}^{+}\right)$for data and generic MC samples where $m_{\mathrm{ES}}>5.20 \mathrm{GeV}$ and $|\Delta E|<0.2 \mathrm{GeV}$. The generic $B^{0} \bar{B}^{0}, B^{+} B^{-}, c \bar{c}$ and uds $\mathrm{MC}$ samples are individually scaled to have integrated luminosities equal to the data sample. . . . . . . . . . . . . . . . . .

8.1 The Dalitz Distribution of $B^{-} \rightarrow \Lambda_{c}^{+} \bar{\Lambda}_{c}^{-} K^{-}$in the signal region $5.27 \mathrm{GeV}<$ $m_{\mathrm{ES}}<5.29 \mathrm{GeV}$, where $\Lambda_{c}^{+} \rightarrow p^{+} K^{-} \pi^{+}$. (a) $m^{2}\left(\bar{\Lambda}_{c}^{-} K^{-}\right)$vs. $m^{2}\left(\Lambda_{c}^{+} \bar{\Lambda}_{c}^{-}\right)$, (b) $m^{2}\left(\bar{\Lambda}_{c}^{-} K^{-}\right)$vs. $m^{2}\left(\Lambda_{c}^{+} K^{-}\right)$. The large black points are the events reconstructed from data, and the small red points are the reconstructed signal events generated with a phase space model. The black curves are the boundary of the Dalitz plots restricted by the four-momentum conservation. Mass constraints on $B$ is not applied, so points can lie outside the boundary. . . . . . . . . . . . . . .

8.2 The projections $m\left(\Lambda_{c}^{+} \bar{\Lambda}_{c}^{-}\right)$(a), $m\left(\bar{\Lambda}_{c}^{-} K^{-}\right)$(b) and $m\left(\Lambda_{c}^{+} K^{+}\right)$(c) for the same events used in fig 8.1. The black curve is reconstructed from data, the red curve represents the distribution of reconstructed signal events generated with a phase space model, and the blue hatched region represents the background distribution estimated from the events in the region of $m_{\mathrm{ES}}<5.26 \mathrm{GeV}$. . . . . . . . . . . . . . . . . . . . . . . . 164 


\section{CHAPTER 1 INTRODUCTION}

\subsection{Standard Model}

Elementary particle physics is a sub-field of physics science probing the deepest secrets of nature - What is the world made of and how do the fundamental elements interact? Decades of hard work by experimental and theoretical particle physicists led to one of the best scientific achievement of the twentieth century - the Standard Model of particle physics. It covers our current best understanding of the fundamental components of matter and their interactions and has successfully predicted the outcome of a large variety of experiments in particle physics.

\subsubsection{Fundamental particles}

According to the Stanford Model, the fundamental constituents [1] of matter are composed of twelve spin $\frac{1}{2}$ fermions grouped in three generations of quarks (two flavors of quarks in one generation/group)

$$
\left(\begin{array}{l}
u \\
d
\end{array}\right)\left(\begin{array}{l}
c \\
s
\end{array}\right)\left(\begin{array}{l}
t \\
b
\end{array}\right)
$$

and three generations of leptons (one charged lepton and one corresponding neutrino in one generation/group)

$$
\left(\begin{array}{c}
e \\
\nu_{e}
\end{array}\right)\left(\begin{array}{c}
\mu \\
\nu_{\mu}
\end{array}\right)\left(\begin{array}{c}
\tau \\
\nu_{\tau}
\end{array}\right)
$$


In the frame of the standard model, each of these fermions has a corresponding anti-particle (another twelve fundamental fermions) which are also grouped in three similar generations. A summary of the basic properties of these fermions are listed in Table 1.1.

Table 1.1: Properties of fermions in the Standard Model.

\begin{tabular}{clllll}
\hline \hline Name & $\mathrm{m}\left(\mathrm{MeV} / \mathrm{c}^{2}\right)$ & charge & spin & color & isospin \\
\hline $\mathrm{u}$ & $1.5 \sim 3.0$ & $\frac{2}{3}$ & & & $\frac{1}{2}$ \\
$\mathrm{~d}$ & $3 \sim 7$ & $-\frac{1}{3}$ & & & $\frac{1}{2}$ \\
$\mathrm{c}$ & $(1.3 \pm 0.1) \times 10^{3}$ & $\frac{2}{3}$ & $\frac{1}{2}$ & 3 & 0 \\
$\mathrm{~s}$ & $95 \pm 25$ & $-\frac{1}{3}$ & & & 0 \\
$\mathrm{t}$ & $(172-174) \times 10^{3}$ & $\frac{2}{3}$ & & & 0 \\
$\mathrm{~b}$ & $(4.2-4.7) \times 10^{3}$ & $-\frac{1}{3}$ & & \\
\hline$e^{-}$ & 0.511 & -1 & & & \\
$\nu_{e}$ & $<0.003$ & 0 & & \\
$\mu^{-}$ & 105.7 & -1 & & & \\
$\nu_{\mu}$ & $<0.19$ & 0 & $\frac{1}{2}$ & & \\
$\tau^{-}$ & 1777 & -1 & & & \\
$\nu_{\tau}$ & $<18.2$ & 0 & & & \\
\hline
\end{tabular}

Note: The anti-particle has same mass as the particle, but opposite quantum numbers.

All the up-type quarks - $\mathrm{u}(\mathrm{p}), \mathrm{c}(\mathrm{harm}), \mathrm{t}(\mathrm{op})$ - carry an electric charge of $+\frac{2}{3}$, and the down-type quarks - d(own), s(trange), b(ottom) - carry an electric charge of $-\frac{1}{3}$. The first generation quarks $\mathrm{u}$ and $\mathrm{d}$ form an isospin (I) doublet with $\mathrm{I}$ of $1 / 2$, with the projection $I_{z}\left(\frac{1}{2},-\frac{1}{2}\right)$, corresponding to the two charged states of the quark doublet. (This has a historical beginning, with proton and neutron, the two nucleons, considered as the two charged states of a single nucleonic state under the 
$\mathrm{SU}(2)$ symmetry.) While the $\mathrm{c}, \mathrm{s}, \mathrm{b}$, and t quarks have $\mathrm{I}=0$ assigned to them, each has its own identifying quantum numbers - S for s quark, C for c quark, B for b quark and $\mathrm{T}$ for $\mathrm{t}$ quark. Quarks are also assigned another important quantum number - "color", denoted symbolically by R(red), G(green), and B(blue), which, of course, have no connection to pigments. The electric charge is conserved all the time, but the other quantum numbers, like I,S,C, T, B are only conserved in some interactions. In reality, no free quarks have been observed, they are supposed to be confined inside the so-called "hadrons" which are bound states of quarks (or anti-quarks) held together by strong interactions, non-Abelian gauge interactions mediated by "gluons". The bound state of a quark and an anti-quark is called a meson, and the bound state of three quarks is called a baryon. Bound states with quark and/or anti-quark combinations outside this quark model, are called exotics [2][3] [4], such as the pentaquark[5][6]. Examples of exotic mesons include hybrid mesons, glueballs and tetraquarks. These are hotly debated current topics.

Each lepton generation is assigned a lepton flavor (LF) number: $L_{e}, L_{\mu}, L_{\tau}$, which is equal to +1 for each lepton and -1 for its anti-particle; the lepton number of non-leptonic particle is assigned as 0 . Generally, in standard model, the lepton family number is conserved in particle decays. But observation of neutrino oscillations [7][8] where neutrinos of one generation change into neutrinos of another generation, show clear violation of LF conservation. However, this is very small, and for most purposes we can still consider the LF as conserved, especially for the charged leptons.

So far, all neutrinos were observed to have their spins anti-parallel to their 
momenta (helicity -1, called left-handed), and anti-neutrinos to have spins parallel to their momenta (helicity +1 , called right-handed); however, since the neutrino oscillations indicate neutrinos to be no longer massless, many revised theories of electro-weak interactions include left-handed as well as right-handed neutrinos.

\subsubsection{Fundamental interactions}

Particles interact via four fundamental forces: the Gravitational, the Weak, the Electromagnetic, and the Strong. The quantization of gravity is still somewhat illusive. Moreover, at the subatomic level, the gravitational force is so weak that it is safely ignored. The Standard Model thus incorporates the Strong, Electromagnetic and the Weak interactions. It also shows (and is experimentally proven) that the Electromagnetic and and the Weak interactions are but the low energy manifestations of the unified Electroweak interactions; this, in turn, requires the local gauge invariance with the gauge group $S U(3) \times S U(2) \times U(1)$. It also infers each interaction to have its own field mediator - the gauge bosons. The electromagnetic interaction described by the Quantum Electrodynamics (QED) is part of the gauge group U(1), and the gauge boson is the photon. The Strong interaction is described by the Quantum Chromodynamics (QCD) with its gauge group as $\mathrm{SU}(3)_{c}$, (c for color) and the gauge bosons are eight gluons which are bi-colored and transform one colored quark into a different colored one in interactions between quarks. The gluons are responsible for binding the quarks together to form hadrons; leptons do not carry color and therefore can not participate in the Strong interactions. The Weak interactions, described by 
the Electroweak theory of Standard model is part of the gauge group $U(1) \times S U(2)$, and the $W^{ \pm}$and $Z^{0}$ bosons are the mediators of Weak force. They have already been observed in experiments [9][10]. The quantum numbers of the gauge bosons are listed in Table 1.2. The photons and the gluons are massless, but the $\mathrm{W}$ and the $\mathrm{Z}$ bosons are very massive, which posed a problem for the gauge theory. To circumvent it, a spontaneously broken gauge symmetry mechanism - known as the Higgs mechanism was introduced (after Peter Higgs....). The Higgs boson, a scalar field, is believed to generate masses of all of the fundamental particles by interacting with the corresponding fields. The Higgs boson is yet to be observed.

Quantum Chromodynamics, the theory of the Strong interactions, explains why free quarks are not observed in nature by 'confinement'. All observable particles of nature has to be color neutral, ie., they have to be either colorless (like redantired) or color white (red-blue-green). That means the color lines can not be broken. The quarks are confined with other quarks (or antiquarks) by the strong force into the color-neutral hadrons. The force between quarks embedded in hadron increases as the distance between them increases, or asymptotically converges to zero at very short distances, like deep inside the hadrons. This phenomenon is called Asymptotic freedom in QCD. Hence, no quark can be found individually. At low energies where the Strong force becomes extremely strong, the hadronic interactions can not be calculated by the perturbative methods and poses a serious challenge, since almost all interactions are observed through the eventual cascading low energy manifestations. For precision measurements we need to understand the QCD effects 
they are "wrapped in"; hence it is imperative to have a better understanding of QCD in the lower energy ranges.

Table 1.2: Properties of gauge Bosons in the Standard Model

\begin{tabular}{|r|l|c|c|c|c|c|}
\hline \hline \multicolumn{2}{|c|}{ bosons } & mass $(\mathrm{GeV})$ & spin & charge & range $(\mathrm{cm})$ & strength \\
\hline strong & gluons & 0 & 1 & 0 & $10^{-13}$ & 1 \\
\hline EM & photon $(\gamma)$ & 0 & 1 & 0 & infinite & $10^{-2}$ \\
\hline \multirow{3}{*}{ weak } & $W^{+}$ & 80 & 1 & +1 & $10^{-16}$ & $10^{-6}$ \\
& $W^{-}$ & 80 & 1 & -1 & $10^{-16}$ & $10^{-6}$ \\
& $Z^{0}$ & 91 & 1 & 0 & $10^{-16}$ & $10^{-6}$ \\
\hline
\end{tabular}

When hadrons decay by weak interactions, the flavor of the quarks are modified. The strong interactions do not change the flavor of the quarks (except through quark-anitiquark annihilation), ie., flavor is conserved in the strong interactions. The strong and the weak interaction eigenstates of the quarks are described by a complex $3 \times 3$ unitary matrix, known as the Cabibbo-Kobayayashi-Maskawa (CKM) matrix:

$$
\left(\begin{array}{c}
d^{\prime} \\
s^{\prime} \\
b^{\prime}
\end{array}\right)=\left(\begin{array}{ccc}
V_{u d} & V_{u s} & V_{u b} \\
V_{c d} & V_{c s} & V_{c b} \\
V_{t d} & V_{t s} & V_{t b}
\end{array}\right)\left(\begin{array}{c}
d \\
s \\
b
\end{array}\right)
$$

Where the ( $\mathrm{d} \mathrm{s} \mathrm{b})$ represents the strong interaction eigenstates, the $\left(d^{\prime} s^{\prime} b^{\prime}\right)$ represent the weak force eigenstates, and $V_{i j}$ is the probability of the $i$ th quark decaying into the 
$j$ th quark via the weak interactions. This matrix was introduced for three generations of quarks by Makoto Kobayashi and Toshihide Maskawa [12], adding one generation to the matrix by Nicola Cabibbo [11]. The diagonal elements of the matrix are close to unity, showing that the up-type quarks prefer mostly to decay into the down-type quark in the same generation; the non-zero off-diagonal elements represent the uptype quarks relatively rarer decay into down-type quarks in different generations, like $b \rightarrow c$. The full information on the amplitudes of the CKM elements is summarized

$$
\left|V_{C K M}\right|=\left(\begin{array}{cccc}
0.9739 \sim 0.9751 & 0.221 \sim 0.227 & 0.0029 \sim 0.0045 \\
0.221 \sim 0.227 & 0.9730 \sim 0.9744 & 0.039 \sim 0.044 \\
0.0048 \sim 0.014 & 0.037 \sim 0.043 & 0.9990 \sim 0.9992
\end{array}\right)
$$

These values are derived from both direct measurement and three generation unitary.

In principle, the nine matrix elements (because it is unitary) can be reduced to four independent parameters, which includes three mixing angles and one CPviolating (the breaking of charge conjugate, $\mathrm{C}$, and and parity, $\mathrm{P}$, symmetries, specifically, CP) complex phase. Among several parameterizations, the commonly used one is the Wolfenstein parameterization [13] which expresses the CKM matrix elements in terms of four real, independent parameters $(A, \lambda, \rho$ and $\eta)$, and demonstrates the hierarchical structure of the elements at a glance, very useful in $B$ physics studies.

$$
V_{C K M}=\left(\begin{array}{ccc}
1-\lambda^{2} / 2 & \lambda & A \lambda^{3}(\rho-i \eta) \\
-\lambda & 1-\lambda^{2} / 2 & A \lambda^{2} \\
A \lambda^{3}(1-\rho-i \eta) & -A \lambda^{2} & 1
\end{array}\right)+O\left(\lambda^{4}\right)
$$


In this parameterization, $\lambda=\sin \theta_{c}$, where $\theta_{c}$ is the well-known Cabibbo angle, is small $(\lambda \simeq 0.23)$. The complex phase $(\rho, \eta)$, which is responsible for $\mathrm{CP}$ violation in the Standard Model,. The CKM matrix elements can be depicted in the form of a triangle, shown in Fig 1.1, representing the unitarity relations, and denoted by $\alpha, \beta$ and $\gamma$ :

$$
\begin{aligned}
\alpha & =\arg \left(-\frac{V_{t d} V_{t b}^{*}}{V_{u d} V_{u b}^{*}}\right) \\
\beta & =\arg \left(-\frac{V_{c d} V_{c b}^{*}}{V_{t d} V_{t b}^{*}}\right) \\
\gamma & =\arg \left(-\frac{V_{u d} V_{u b}^{*}}{V_{c d} V_{c b}^{*}}\right)=\pi-\alpha-\beta
\end{aligned}
$$

A non-zero area of the triangle (non-vanishing phase) indicates CP-violation and any trivial value of $\alpha, \beta$ and $\gamma\left(0^{\circ}\right.$ or $\left.180^{\circ}\right)$ would mean $\mathrm{CP}$ conservation.

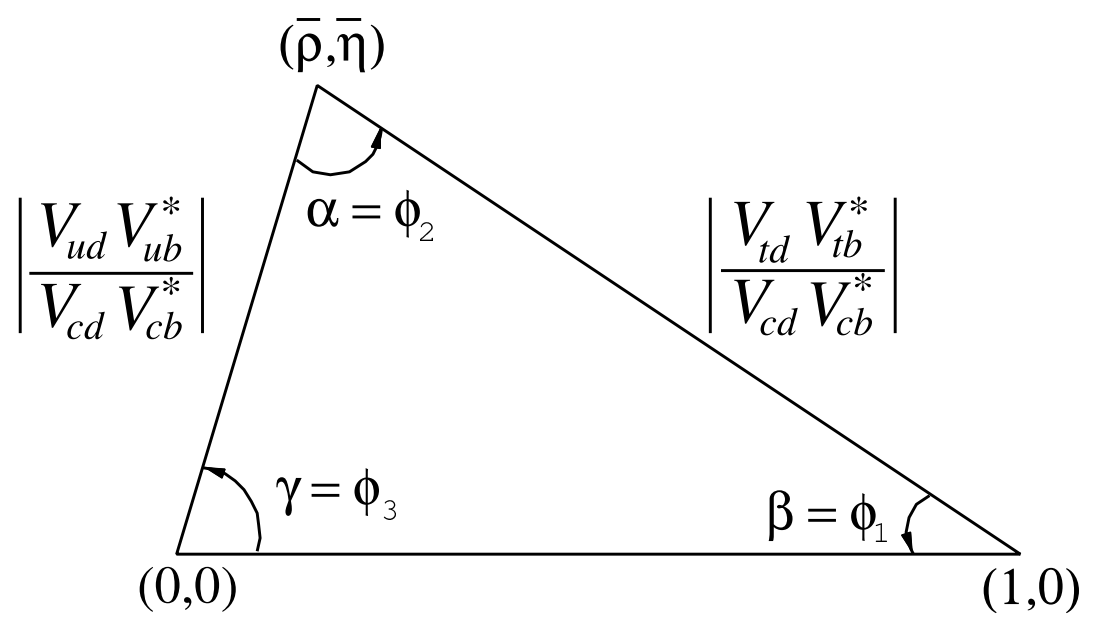

Figure 1.1: The unitary triangle with the definition of $\rho, \eta$, and CKM angles $\alpha, \beta$ and $\gamma$. 
CP-violation was first observed in the neutral Kaon-physics; but because the phase angle to be measured exhibits itself via the matrix elements $V_{c s}$ and $V_{c d}$, which are expressed in higher orders of the parameter $\lambda\left(\mathcal{O}\left(\lambda^{5}\right)\right)$, it is very difficult to disentangle the CP-violation quantitatively because of the much larger QCD effects.

\subsubsection{Perspectives on the Standard Model}

The Standard Model consists of 18 parameters [14] (some suggest more free parameters): three coupling constants $\left(\alpha_{s}=g_{3}^{2} / 4 \pi, \alpha_{E M}=\left(e^{2} / 4 \pi\right)\left(e=g \cdot \sin \theta_{w}\right)\right.$, and $\left.\sin ^{2} \theta_{w}=\left(g^{\prime}\right)^{2} /\left(g^{2}+\left(g^{\prime}\right)^{2}\right)\right)$, nine charged fermion masses, four parameters describing the mass mixing matrix between $u$ and d-type quarks of different generations (the CKM matrix), and two parameters describing the Higgs sector, which can be taken to be the vacuum expectation value of the original Higgs field and the mass of the Higgs boson after the symmetry breaking. The first 17 parameters of these have been measured to varying levels of precision, with only bounds on the Higgs mass, the eighteenth parameter.

All of the experiments to date confirm the Standard Model predictions. However, the model falls short of explaining many questions, such as: the three generational hierarchy and the lepton-quark symmetry, the specific masses and the charges, the overwhelming matter-antimatter difference observed in our universe, dark matter and dark energy. So, clearly at some point, the Standard Model breaks down, much like Quantum Mechanics and Relativity supersede Newtonian Mechanics. To find a window into what lies beyond the Standard Model, many lower energy, high statistics, 
insightful experiments were designed; BABAR is such a flagship experiment.

\subsection{Charmed baryons}

\subsubsection{A short history of the charm quark}

In November 1974, two experiments [17][18] simultaneously announced the discovery of the $J / \psi$, a meson with a mass of about $3.1 \mathrm{GeV}$ and a narrow width which implied a much longer lifetime than other massive mesons. This particle was interpreted as the charmonium, a bound state of $c \bar{c}$ - heralding the discovery of the charm quark. This interpretation was later confirmed with the discoveries of the charmed hadrons (open charm), namely, $D^{0(+)}(\bar{c} u(c \bar{d}))$ mesons [19][20] in 1976. The first evidence of charmed baryon production [21] arose from the study of a neutrino interaction in the Brookhaven National Lab (BNL) 7-foot bubble chamber in 1975. Later on, this particle was confirm as a $\Sigma_{c}^{++}$particle followed by the decay to $\Lambda_{c}^{+}$. The first completely reconstructed charmed baryon has been the $\Lambda_{c}^{+}(2284)$ from the decay $\Lambda_{c}^{+} \rightarrow p^{+} K^{-} \pi^{+}$by one of the neutrino-beam experiments at CERN.

\subsubsection{Quark model of the charmed baryons}

Charmed baryons are composed of typically one $c$ quark and two lighter quarks ( $\mathrm{u}, \mathrm{d}$, and s). Baryons with two charmed quarks are predicted, but has not yet been observed. The spectroscopy of the ground state charmed baryons was mapped out in 1974 [16] with the SU(4) flavor symmetry group. Charmed baryon multiplets are thus belong to $4 \times 4 \times 4=20+20_{1}{ }^{\prime}+20_{2}^{\prime}+\overline{4}$. Although the mass of the $c$ quark is much heavier, breaking the flavor symmetry rather badly, the descriptions still apply. 
However, predictions of the masses based on the flavor SU(4) symmetry might not be applicable.

A graphical representation of the $\mathrm{SU}(4)$ baryons is shown in Fig 1.2, where the strangeness quantum number of the baryons $S=0,1,2,3$ increases from back to front, $I=0,1 / 2,1,3 / 2$ with $I_{3}$ increasing from let to right, and $C=0,1,2,3$ from bottom to top. The charmless baryons $\left(p, \Lambda^{0}, \Sigma, \Xi\right)$ reside on the bottom layer, while the singly charmed baryons are on the second layer of these multiplets. One 20 symmetric multiplet with symmetric spin arrangement $\frac{3}{2}$, and one 20 multiplet of mixed symmetry $20^{\prime}$ multiplet with symmetric spin $\frac{1}{2}$ form the symmetric spin-flavor ground state $(L=0)$ in Figure $1.2(\mathrm{a}),(\mathrm{b})$.
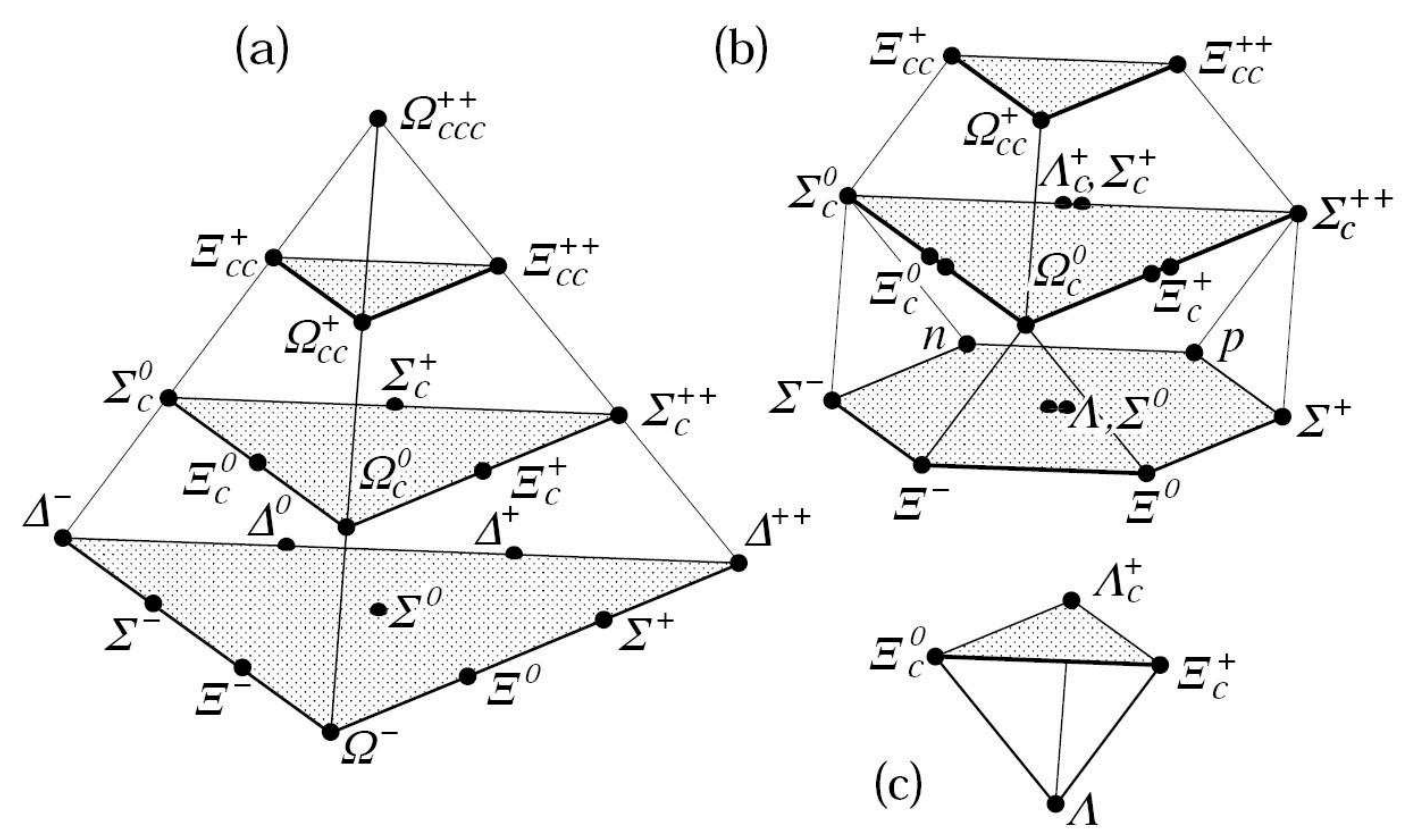

Figure 1.2: $\mathrm{SU}(4)$ baryon multiplets which show all the $\mathrm{L}=0$ baryons in a four-quark system. 
The wave function of a baryon consists of four parts: spatial,spin, flavor, and, color, as expressed below.(For ease of explanation, only $\mathrm{SU}(3)$ is demonstrated.)

$$
\psi=\psi(\text { space }) \psi(\text { spin }) \psi(\text { flavor }) \psi(\text { color })
$$

As a fermion, a baryon follows the Fermi-Dirac statistics (Pauli Exclusion Rule), and therefore its wave-function has to be anti-symmetric. Since all the naturally occurring particles are colorless, naively, the color part of the wave function is a singlet, which is completely anti-symmetric, SU(3) color singlet. The space part of the wave-function is dependent on the Orbital angular momenta of the three-body system. It is always symmetric for the ground-state baryons, where the position of any two quarks are interchangeable, because there is no angular dependence at all $(L=0)$. This means that the combination of the spin and the flavor part of the baryon wave function, $\psi($ spin $) \psi($ flavor $)$, has to be symmetric for the ground state.

In the ground state, the baryon spin is simply the addition of three quark spins $\left(\frac{1}{2}\right)$ with no orbital angular momentum. Writing the decomposition in terms of the multiplicities of the spin states, we get

$$
2 \times 2 \times 2=\left(3_{S}+1_{A}\right) \times 2=4_{S}+2_{M_{S}}+2_{M_{A}}
$$

The subscripts in the mixed symmetry $\operatorname{doublets}\left(M_{S}, M_{A}\right)$ indicate whether the spin part of the wave function is symmetric or antisymmetric under interchange of the first two quarks. The spin wave function of the four $S=\frac{3}{2}$ spin states are fully symmetric. To enumerate the baryons expected in the quark model, we combine the SU(4) flavor decomposition with the $\mathrm{SU}(2)$ spin decomposition. Considering 
the product symmetries, we are lead to assign the $(\mathrm{SU}(4), \mathrm{SU}(2))$ multiplets to the following categories:

$$
\begin{array}{cc}
\mathbf{S}: & (20,4)+\left(20^{\prime}, 2\right) \\
\mathbf{M}_{\mathbf{S}}: & (20,2)+\left(20^{\prime}, 4\right)+\left(20^{\prime}, 2\right)+(\overline{4}, 2) \\
\mathbf{M}_{\mathbf{A}}: & (20,2)+\left(20^{\prime}, 4\right)+\left(20^{\prime}, 2\right)+(\overline{4}, 2) \\
\mathbf{A}: & (\overline{4}, 4)+\left(20^{\prime}, 2\right)
\end{array}
$$

only the combinations in the $\mathbf{S}$ category can form ground states.

Therefore the ground-state charmed baryons fall into two representations of flavor $\mathrm{SU}(3)$. If the charmed baryon state vector is antisymmetric under interchange of the two light quark flavors then it is in the $\overline{3}$ representation and if it is symmetric under interchange of the two light quark flavors then it is in the 6 representation. In the non-relativistic potential (quark) model the spin-flavor state vectors for the ground-state $J^{P}=\frac{1}{2}^{+}$baryons in the $\overline{3}$ representation of $\mathrm{SU}(3)$ are

$$
\begin{aligned}
& \mid \Lambda_{c}^{+}>=\frac{1}{2}(|c \uparrow u \uparrow d \downarrow>-| c \uparrow u \downarrow d \uparrow>-|c \uparrow d \uparrow u \downarrow>+| c \uparrow d \downarrow u \uparrow>) \\
& \mid \Xi_{c}^{+}>=\frac{1}{2}(|c \uparrow u \uparrow s \downarrow>-| c \uparrow u \downarrow s \uparrow>-|c \uparrow s \uparrow u \downarrow>+| c \uparrow s \downarrow u \uparrow>) \\
& \mid \Xi_{c}^{0}>=\frac{1}{2}(|c \uparrow d \uparrow s \downarrow>-| c \uparrow d \downarrow s \uparrow>-|c \uparrow s \uparrow d \downarrow>+| c \uparrow s \downarrow d \uparrow>)
\end{aligned}
$$

and the spin-flavor state vectors for the ground-state $J^{P}=\frac{1}{2}^{+}$baryons in the 
6 representation of $\mathrm{SU}(3)$ are

$$
\begin{aligned}
\mid \Sigma_{c}^{++}>= & \frac{1}{\sqrt{6}}(2|c \downarrow u \uparrow u \uparrow>-| c \uparrow u \uparrow u \downarrow>-\mid c \uparrow u \downarrow u \uparrow>) \\
\mid \Sigma_{c}^{+}>= & \frac{1}{\sqrt{12}}(|c \downarrow u \uparrow d \uparrow>-| c \uparrow u \uparrow d \downarrow>-\mid c \uparrow u \downarrow d \uparrow> \\
& +2|c \downarrow d \uparrow u \uparrow>-| c \uparrow d \uparrow u \downarrow>-\mid c \uparrow d \downarrow u \uparrow>) \\
\mid \Sigma_{c}^{0}>= & \frac{1}{\sqrt{6}}(2|c \downarrow d \uparrow d \uparrow>-| c \uparrow d \uparrow d \downarrow>-\mid c \uparrow d \downarrow d \uparrow>) \\
\mid \Xi_{c}^{\prime+}>= & \frac{1}{\sqrt{12}}(|c \downarrow u \uparrow s \uparrow>-| c \uparrow u \uparrow s \downarrow>-\mid c \uparrow u \downarrow s \uparrow> \\
& +2|c \downarrow s \uparrow u \uparrow>-| c \uparrow s \uparrow u \downarrow>-\mid c \uparrow s \downarrow u \uparrow>) \\
\mid \Xi_{c}^{\prime 0}>= & \frac{1}{\sqrt{12}}(|c \downarrow d \uparrow s \uparrow>-| c \uparrow d \uparrow s \downarrow>-\mid c \uparrow d \downarrow s \uparrow> \\
& +2|c \downarrow s \uparrow d \uparrow>-| c \uparrow s \uparrow d \downarrow>-\mid c \uparrow s \downarrow d \uparrow>) \\
\mid \Omega_{c}^{0}>= & \frac{1}{\sqrt{6}}(2|c \downarrow s \uparrow s \uparrow>-| c \uparrow s \downarrow s \uparrow>-\mid c \uparrow s \uparrow s \downarrow>)
\end{aligned}
$$

The lowest lying $J^{P}=3 / 2^{+}$charmed baryons are also i the 6 representation of $\mathrm{SU}(3)$.

Their spin-flavor state vectors are

$$
\begin{aligned}
\mid \Sigma_{c}^{*++}> & =\mid c \uparrow u \uparrow u \uparrow> \\
\mid \Sigma_{c}^{*+}> & =\frac{1}{\sqrt{2}}(|c \uparrow u \uparrow d \uparrow>+| c \uparrow d \uparrow u \uparrow>) \\
\mid \Sigma_{c}^{* 0}> & =\mid c \uparrow d \uparrow d \uparrow> \\
\mid \Xi_{c}^{*+}> & =\frac{1}{\sqrt{2}}(|c \uparrow u \uparrow s \uparrow>+| c \uparrow s \uparrow u \uparrow>) \\
\mid \Xi_{c}^{* 0}> & =\frac{1}{\sqrt{2}}(|c \uparrow d \uparrow s \uparrow>+| c \uparrow s \uparrow d \uparrow>) \\
\mid \Omega_{c}^{* 0}> & =\mid c \uparrow s \uparrow s \uparrow>
\end{aligned}
$$

The masses and $J^{P}$ 's of the ground-state charmed baryons, 15 in total, are listed in Table 1.3. 
Table 1.3: Properties of the $\mathrm{L}=0$ charmed baryons

\begin{tabular}{|c|c|c|c|}
\hline Baryon & content & Mass $(\mathrm{MeV})$ & $\mathrm{I}\left(J^{P}\right)$ \\
\hline$\Lambda_{c}^{+}$ & $c(u d)_{a}$ & $2286.46 \pm 0.14$ & $0\left(\frac{1}{2}^{+}\right)$ \\
\hline$\Sigma(2455)_{c}^{0}$ & $c d d$ & $2453.76 \pm 0.18$ & $1\left(\frac{1}{2}^{+}\right)$ \\
\hline$\Sigma(2455)_{c}^{+}$ & $c(u d)_{s}$ & $2452.9 \pm 0.4$ & $1\left(\frac{1}{2}^{+}\right)$ \\
\hline$\Sigma(2455)_{c}^{++}$ & сии & $2454.02 \pm 0.18$ & $1\left(\frac{1}{2}^{+}\right)$ \\
\hline$\Sigma(2520)_{c}^{0}$ & $c d d$ & $2518.0 \pm 0.5$ & $1\left(\frac{3}{2}^{+}\right)$ \\
\hline$\Sigma(2520)_{c}^{+}$ & cud & $2517.5 \pm 2.3$ & $1\left(\frac{3}{2}^{+}\right)$ \\
\hline$\Sigma(2520)_{c}^{++}$ & сии & $2518.4 \pm 0.6$ & $1\left(\frac{3}{2}^{+}\right)$ \\
\hline$\Xi_{c}^{0}$ & $c(s d)_{a}$ & $2471.0 \pm 0.4$ & $\frac{1}{2}\left(\frac{1}{2}^{+}\right)$ \\
\hline$\Xi_{c}^{+}$ & $c(s u)_{a}$ & $2467.9 \pm 0.4$ & $\frac{1}{2}\left(\frac{1}{2}^{+}\right)$ \\
\hline$\Xi_{c}^{\prime 0}$ & $c(s d)_{s}$ & $2578.0 \pm 2.9$ & \\
\hline$\Xi_{c}^{\prime+}$ & $c(s u)_{s}$ & $2575.7 \pm 3.1$ & $\frac{1}{2}\left(\frac{1}{2}^{+}\right)$ \\
\hline$\Xi_{c}^{* 0}$ & $c s d$ & $2646.1 \pm 1.2$ & $\frac{1}{2}\left(\frac{3}{2}^{+}\right)$ \\
\hline$\Xi_{c}^{*+}$ & csu & $2646.6 \pm 1.4$ & $\frac{1}{2}\left(\frac{3}{2}^{+}\right)$ \\
\hline$\Omega_{c}^{0}$ & css & $2697.5 \pm 2.6$ & $0\left(\frac{1}{2}^{+}\right)$ \\
\hline$\Omega_{c}^{\prime 0}$ & $c s s$ & observed & $0\left(\frac{3}{2}^{+}\right)$ \\
\hline
\end{tabular}

Note: The subscript a and s denote anti-symmetric and symmetric flavor index combination. The spin and parity are derived from theoretical prediction; no measurement exist to date.

\subsubsection{Decay of charmed baryons}

Four spin $\frac{1}{2}$ charmed baryons - $\Lambda_{c}^{+}(c u d), \Xi_{c}^{0}(c s d), \Xi_{c}^{+}(c s u)$, and $\Omega_{c}^{0}(c s s)$ - are observed in the weak interaction process of $c \rightarrow s$ decay to light baryons (baryons with only $u, d$, or $s$ quarks. A Feynman diagram of a typical decay of $\Xi_{c}^{0}$ according to the Spectator model, where only the heavy quark participates in the interaction, is shown in Figure 1.3. All other charmed baryons can decay via strong or EM interactions with a pion or a photon emission to these four states as shown in Figure 1.4. Higher (excited) states of charmed baryons massive enough to decay into a charmed meson 


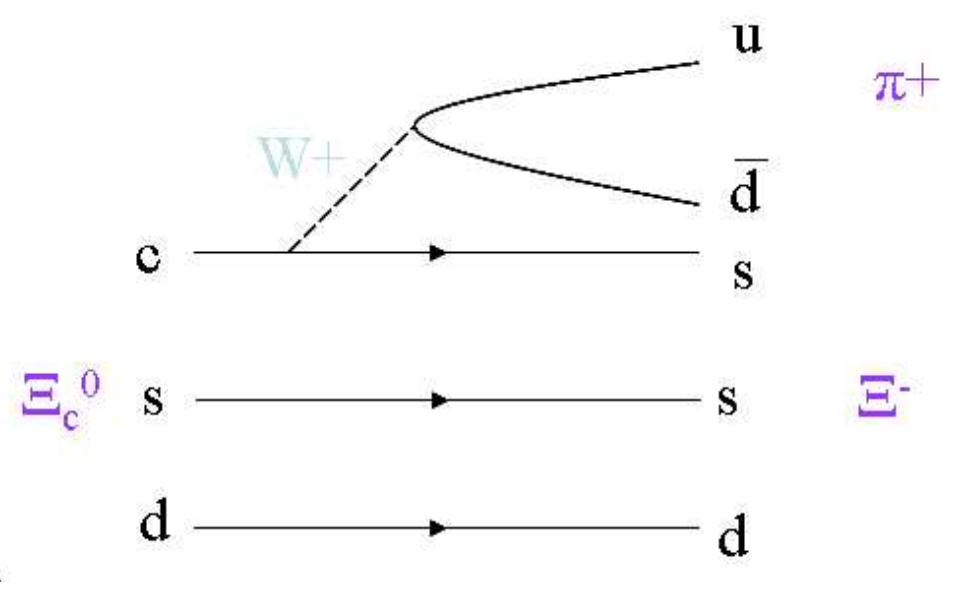

Figure 1.3: Tree level Feynman diagram illustrating the decay mechanism of $\Xi_{c}^{0} \rightarrow$ $\Xi^{-} \pi^{+}$.

and a baryon $(D \mathrm{p})$ have also been observed recently by $B A B A R$.

A lot remains to be explored in the charmed baryon sector. The charmed baryon sector is the richest quark spectroscopy available to study. Because there are more possibilities for orbital angular excitations of the three quark system,there are more states compared with mesons. Also, these excited states are fairly narrow, with small mass difference between these and ground states. The weak decays of charmed baryons provide a very rich phenomenology for probing HQE. Heavy quark expansions (HQE) have been developed into a mature theoretical technology for treating decays of heavy flavor hadrons. By employing the operator product expansion [23], inclusive observables like lifetimes and total semileptonic widths can be expressed in inverse powers of the heavy quark mass. In addition, absolute branching fraction are needed for exclusive and inclusive decays of charm baryons as an engineering input for other 


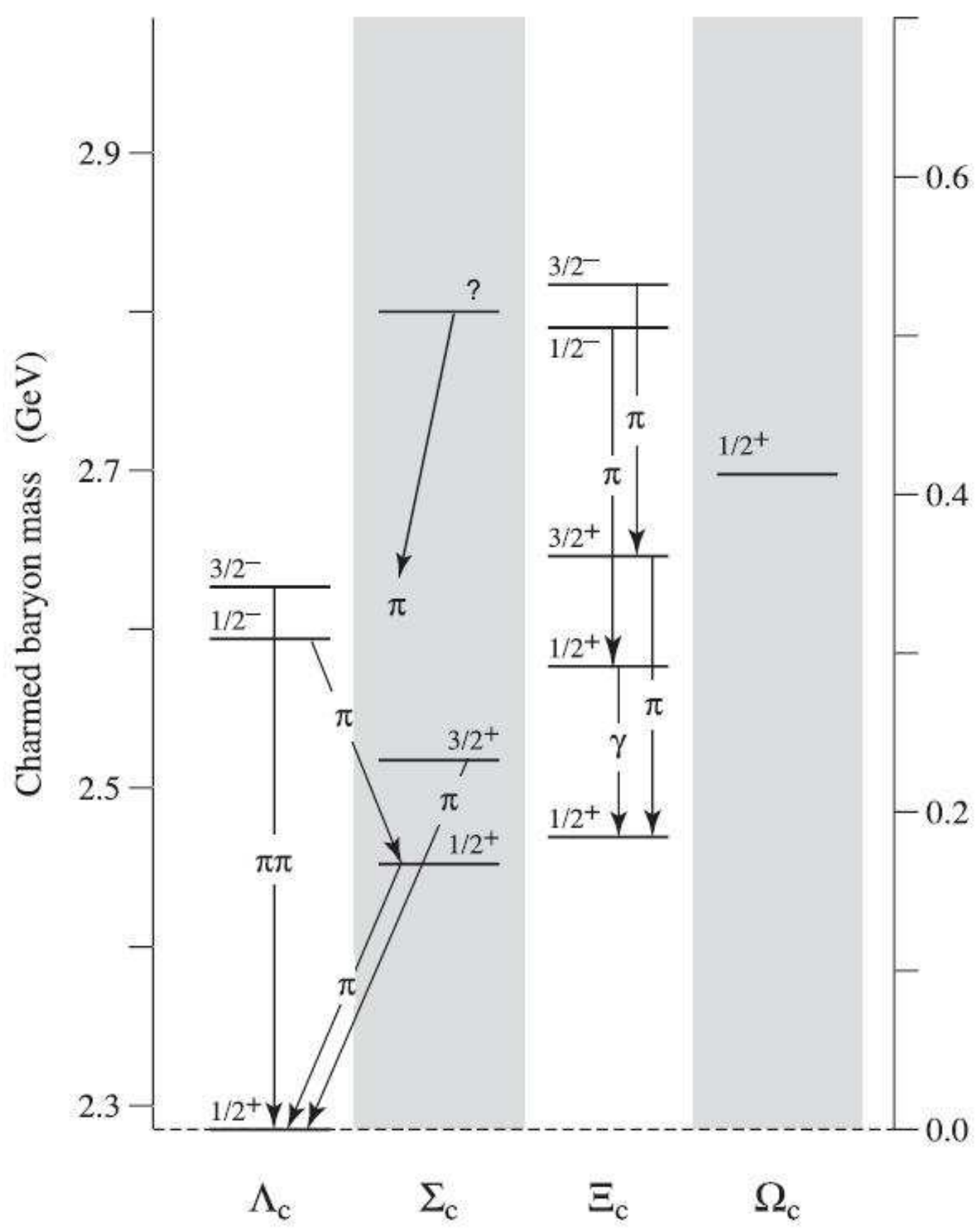

Figure 1.4: Mass spectrum for known charmed baryon. Labels along the x-axis show the quark content of charmed baryons (no s quark, one s quark, two s quarks from left to right. The $J^{P}$ s are theoretical prediction, and no measurement exists. 
studies in B decays, for example the charm counting in $B$ meson decay. The final states in $\Lambda_{c}^{+}$and $\Xi_{c}$ decays can shed new light on the spectroscopy of light flavour baryons.

\subsection{B meson physics}

$B$-physics in $B A B A R$ is the study of the bottom quark - $b$ through $B$-mesons, the bound states of a $b$ quark with a lighter anti-quark. At the BABAR experimental energy, only the $B^{-}(b \bar{u}), \bar{B}^{0}(b \bar{d})$ are produced, and they are referred to $B$ meson. They can only decay by weak interactions, although they are bound by strong force. Since the discovery and the following experimental accessibility of the $b$-quark, $B$-physics has become a major field of study in particle physics, because the b-quark bound states are the only third generation quark ( $t$-quark is too heavy and decays before forming a bound state), they provide a unique opportunity to measure the couplings between the different quarks - the CKM matrix elements $V_{c b}, V_{u b}, V_{t d}$ and $V_{t s}$, thereby to test the Standard Model (SM); if the measured values of the SM parameters, extract in various ways, turn out to be inconsistent, not only will it prove the need for physics beyond Standard Model, but also where to look for hints for beyond the SM. But the QCD effects constitute a serious obstacle, because all of the b-quark decay products are "seen through" the QCD effects, even though the weak interaction quark-decay parts can be calculated accurately. Nevertheless, the $B$-mesons provide a wonderful laboratory for test and development of Quantum Chromo dynamics (QCD), since to a large extent the b-quark decays allow perturbative calculations because of its high 
mass.

\subsubsection{Hadronic decays of $B$ meson}

A b-quark has a preference to decay into a $c$ quark than a $u$ quark by emitting a virtual $W$ boson with the $\mathrm{W}$ decaying into a quark-antiquark or lepton-antilepton pair. The first decay is fully hadronic, and the second one is semi-leptonic. Since the leptons don't interact strongly, the semileptonic $B$ meson decays are less affected by the QCD correction. In the hadronic $B$ decay study, the dynamics are inevitably complicated because only $B$ mesons can be studied and not the free b-quark. The light quarks and gluons surrounding the $b$ quark in the $B$-mesons lead to significant corrections to be taken into account because the long distance effects in the strong interactions can not be tackled with perturbative QCD. Also, the gluon exchange may lead to quark rearrangement between initial state and final state. The nonperturbative nature of the bound states, although simple in principle, makes precise extraction of the relevant weak-phase information difficult.

In QCD, the strong coupling constant $\alpha_{s}\left(Q^{2}\right)$ is energy scale dependent, and decreases with higher energy leading to asymptotic freedom. The 'running' of the strong-coupling-constant is expressed as

$$
\alpha_{s}\left(Q^{2}\right)=\frac{12 \pi}{\left(33-2 n_{f}\right) \ln \left(Q^{2} / \Lambda_{Q C D}\right)},
$$

Here $Q$ represents the interaction energy scale, $\Lambda_{Q C D}$ is the energy scale dependent on the experiment (usually between $0.1 \sim 0.5 \mathrm{GeV}$ ) and $n_{f}$ is the number of flavors. Thus, provided the number of quarks $f=16, \alpha_{s}\left(Q^{2}\right)$ would decrease as $Q^{2}$ increases. 
For $Q^{2} \gg \Lambda_{Q C D}^{2}$, the effective coupling is small and perturbative QCD is applicable to the interactions of the quarks and gluons. For $Q^{2} \sim \Lambda^{2}$, however, this is not applicable. In $B$ meson decays, three energy scales are important: QCD confinement constant $\Lambda_{Q C D} \sim 0.2 \mathrm{GeV}, b$ quark mass $m_{b} \sim 4.7 \mathrm{GeV}$, and weak interaction energy $M_{W} \sim 80 \mathrm{GeV}$, which implies $\Lambda_{Q C D} \ll m_{b} \ll M_{W}$.

To deal with this long distance strong interaction, many theoretical approaches and techniques have been developed; however, each of them provide only partial solutions to the problem. Based on the color transparency argument [22], the OPE( operator Product Expansion) [23] method is used to calculate the decay amplitudes with different energy scale. Because $B$ decays involve a wide variety of energy scales, an effective Hamiltonian can be constructed to separate the non-perturbative from the perturbative contributions explicitly, and the transition matrix elements between the initial and the final state can be written as

$$
<f|\mathcal{H}| i>\propto \sum_{k} C_{k}(\mu)<f\left|O_{k}(\mu)\right| i>
$$

where $C_{k}(\mu)$ 's are the perturbatively calculable Wilson [23] coefficient function at $\mu=m_{b}$ and $<f\left|O_{k}(\mu)\right| i>$ are the non-perturbative hadronic matrix elements of the local operators $O_{i}$ which contain the strong interaction effects below the scale $\mu$. Theoretically, the hadronic matrix elements are the most difficult to compute and several methods, namely, QCD sum rule [24], QCD factorization [25] have been used. 


\subsubsection{Inclusive B meson decays to baryons}

Because the $\mathrm{b}$ quark is very massive, the $B$ decays contain a large number of secondary decay products, it is hard to detect all the decay products of a large number the $B$ decays for exclusive measurements. However, inclusive decays of the $B$ mesons are relatively easier to study and yield a lot of information; hence such decays are studied extensively.

Unlike other mesons, $B$ meson can decay into baryons. Because of baryon number conservation, baryons have to be produced in pair in the $B$ meson decays. The ARGUS, CLEO and CLEO-II experiments have observed inclusive production of the $\bar{p}, \Lambda^{0}, \Xi$ and $\Lambda_{c}^{+}$as well as the $\Xi_{c}$ baryons. Recently BABAR has also reported accurate measurements of several inclusive $B \rightarrow$ charmed-baryon (like $\Lambda_{c}^{+}[26], \Xi_{c}^{0}$ [27], and $\left.\Omega_{c}^{0}\right)$. The measured branching fractions are listed in Table 1.4.

Table 1.4: Branching ratio of inclusive $B$ decays to baryons (PDG)

\begin{tabular}{lcc}
\hline \hline Process & Branching fraction (\%) & Experiment \\
\hline$B \rightarrow \Lambda_{c}^{+} / \bar{\Lambda}_{c}^{-}$anything & $6.4 \pm 1.1$ & CLEO \\
$B \rightarrow \bar{\Lambda}_{c}^{-}, p$ anything & $3.6 \pm 0.7$ & CLEO2 \\
$B \rightarrow p \bar{p}$ anything & $8.0 \pm 0.4$ & ARGUS+CLEO \\
$B \rightarrow \Lambda^{0} \bar{\Lambda}^{0}$ anything & $4.0 \pm 0.5$ & ARGUS+CLEO \\
$B \rightarrow$ baryons anything & $6.8 \pm 0.6$ & ARGUS \\
$B \rightarrow p \bar{p}$ anything & $2.47 \pm 0.23$ & ARGUS+CLEO \\
$B \rightarrow \Lambda^{0} \bar{p}, \bar{\Lambda}^{0} p$ anything & $2.5 \pm 0.4$ & ARGUS+CLEO \\
$B \rightarrow \Xi_{c}^{0}, \Xi_{c}^{0} \rightarrow \Xi^{-} \pi^{+}$anything & $0.0193 \pm 0.0030$ & BABAR+CLEO \\
\hline \hline
\end{tabular}

Note: This table does not list all known modes. 
The dominant decays are those induced by a $b \rightarrow c$ transition. In baryonic $B$ decays either one or two charmed baryons are produced depending on whether the W boson transforms into a $u \bar{d}$ or a $c \bar{s}$ pair. With appropriate identification of the decays products, these two decay rates could be measured. The $b \rightarrow u$ transition is suppressed; this would generate charmless baryons. Because baryons are quite heavy, and have to produced in pairs, the baryonic decays of the $B$ mesons can shed light on the underlying decay mechanisms. The soft $\Xi_{c}^{0}$ momentum spectrum in inclusive $\Xi_{c}^{0}$ production in the center-of-mass frame in the process $B \rightarrow \Xi_{c}^{0} X$, measured by $B A B A R[27]$ indicated either $\Lambda_{c}^{+}$production or the presence of significant multi-body component with light charmless baryons in association with the $\Xi_{c}^{0}$.

\subsubsection{Exclusive B meson decays to charmed baryon pairs}

The decays of $B$ mesons into final states which include a charmed baryon pair are studied in this analysis. The corresponding tree level Feynman diagrams are shown in Fig. 1.5. The characteristic features of these diagrams are the production of the second (anti-)charm baryon which can only take place via an internal $W^{-}$ emission in the spectator model, unlike $B \rightarrow D p \bar{p}$ and $B \rightarrow \Lambda_{c}^{+} \bar{p}$ decays, which can proceed via an external emission of a $W^{-}$as well.

The Cabibbo-favored $B$-decays into charm baryon pair reactions are:

- $\bar{B} \rightarrow \Xi_{c}^{+(0)} \bar{\Lambda}_{c}^{-}(n \pi)$

- $\bar{B} \rightarrow \Lambda_{c}^{+} \bar{\Lambda}_{c}^{-} K(\pi)$

- $B^{-} \rightarrow \Omega_{c}^{0} \bar{\Xi}_{c}^{-}$ 

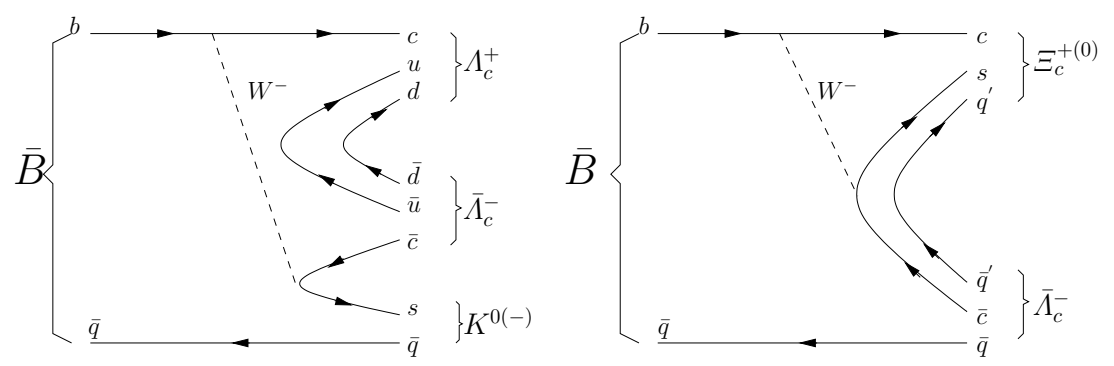

Figure 1.5: Quark level Feynman Diagram for the processes $\bar{B} \rightarrow \Lambda_{c}^{+} \bar{\Lambda}_{c}^{-} \bar{K}$ and $\bar{B} \rightarrow$ $\Xi_{c} \bar{\Lambda}_{c}^{-}$. When $q=d, \bar{B}$ represents $\bar{B}^{0}$, and when $q=u, \bar{B}$ represents $B^{-} . q^{\prime}$ can be either $u$ or $d$ quark.

where $n=0,1,2,3$, depending on the allowed phase space. One or both of the charm baryons could be produced in their excited states, such as $\Xi_{c}^{*(')}, \Lambda_{c}^{*(')}$ or even $\Sigma_{c}$. But all of them decay through either $\Xi_{c}$ or $\Lambda_{c}$ as described in last section. Hence the decay final states, shown in Figure 1.5, include the production of these excited states implicitly. (Excited charm baryons above the $D$ p threshold are known to decay into a charm meson and a baryon; but such a production is not kinematically allowed in $B$ decay here.) The decay $B^{-} \rightarrow \Omega_{c}^{0} \bar{\Xi}_{c}^{-}$needs a pair of relatively heavier $s \bar{s}$ quarks popped up from the vacuum, the decay rate would be much lower than the first two types of decays.

When the $B$ decays into baryons, at least one additional quark pair are generated beside those created in the $b$-quark decay. The three quarks and three anti-quarks can combine in many ways, and therefore it is difficult to develop a good theoretical model for such decays. In the effective Hamiltonian approach, the branching fraction of $B^{-} \rightarrow \Xi_{c}^{0} \bar{\Lambda}_{c}^{-}$, sketched in the Figure 1.5(a), can be calculated with the help of the 
QCD sum rule [28],

$$
\mathcal{B}\left(B^{-} \rightarrow \Xi_{c}^{0} \bar{\Lambda}_{c}^{-}\right) \simeq \frac{G_{F} V_{c b}}{\sqrt{2}}\left(C_{1}-C_{2}\right) I \simeq 0.23 \mathrm{GeV}^{2} G_{F} V_{c b} \simeq 0.1 \%
$$

where $C_{1}-C_{2}$ are Wilson coefficients for the tree-dominated internal W-emission, $G_{F}$ is the weak coupling constant, and I is a non-perturbative term which is calculated by the QCD sum rule [28]. The other phenomenological method [29] also yields similar result. Table 1.5 lists the measurements of such associated production $(b \rightarrow c \bar{c} s)$ of charm baryon pairs in $B$ decays from the Belle experiment in two-body and multibody modes final states [31]. This experimental results are consistent with the

Table 1.5: Bell results on exclusive $B$ decays to charmed baryon pairs

\begin{tabular}{ll}
\hline \hline Process & Branching fraction \\
\hline$B^{-} \rightarrow \Lambda_{c}^{+} \bar{\Lambda}_{c}^{-} K^{-}$ & $\left.\left(6.5 \pm_{0.9}^{1.0}\right) \pm 0.8 \pm 3.4\right) \times 10^{-4}$ \\
$\bar{B}^{0} \rightarrow \Lambda_{c}^{+} \bar{\Lambda}_{c}^{-} K_{s}^{0}$ & $\left.\left(7.0 \pm_{2.3}^{2.0}\right) \pm 1.2 \pm 4.2\right) \times 10^{-4}$ \\
$B^{-} \rightarrow \Xi_{c}^{0} \bar{\Lambda}_{c}^{-}, \Xi_{c}^{0} \rightarrow \Xi^{-} \pi^{+}$ & $\left.\left(4.8 \pm_{0.9}^{1.0}\right) \pm 1.1 \pm 1.2\right) \times 10^{-5}$ \\
$\bar{B}^{0} \rightarrow \Xi_{c}^{+} \bar{\Lambda}_{c}^{-}, \Xi_{c}^{+} \rightarrow \Xi^{-} \pi^{+} \pi^{+}$ & $\left.\left(9.3 \pm_{2.8}^{3.7}\right) \pm 1.9 \pm 2.4\right) \times 10^{-5}$ \\
\hline
\end{tabular}

predictions for $\bar{B} \rightarrow \Xi_{c} \bar{\Lambda}_{c}^{-}$decay within the systematic uncertainties.

However, the same papers [28][29] also predict that the branching fraction for a two-body $B$ decay into a charm baryon pair $B_{c_{1}} B_{c_{2}}$ to be of similar magnitude to decays to a charmed baryon and a light baryon $B_{c_{1}} \bar{N}$, ie.

$$
\mathcal{B}\left(\bar{B} \rightarrow B_{c 1} \bar{B}_{c 2}\right) \approx \mathcal{B}\left(\bar{B} \rightarrow B_{c 1} \bar{N}\right), \text { where } B_{c}=\Sigma_{c}, \Xi_{c}, \Lambda_{c}, \ldots \text { and } N=n, p, \sigma, \Lambda^{0}, \ldots
$$


which is in disagreement with another experimental results from Belle, which is $\mathcal{B}\left(\bar{B} \rightarrow \Xi_{c} \bar{\Lambda}_{c}^{-}\right) \simeq 10^{2} \times \mathcal{B}\left(\bar{B} \rightarrow \Lambda_{c}^{+} \bar{p}\right) \simeq 10^{2} \times \mathcal{B}\left(B^{0} \rightarrow \Sigma_{c}^{0} \bar{p}\right)$. This implies some important dynamical effects exist, because the CKM mixing angles for them are the same in magnitude.

For the three-body decay $B^{-} \rightarrow \Lambda_{c}^{+} \bar{\Lambda}_{c}^{-} K^{-}$, the factorisable amplitude [34] in the weak Hamiltonian approach is given as:

$$
A\left(B^{-} \rightarrow \Lambda_{c}^{+} \bar{\Lambda}_{c}^{-} K^{-}\right)=\frac{G_{F}}{\sqrt{2}} V_{c b} V_{u s}^{*} a_{2}<\Lambda_{c}^{+} \bar{\Lambda}_{c}^{-}|(\bar{c} c)| 0><K^{-}|(\bar{s} b)| B^{-}>
$$

where $\overline{q_{1}} q_{2}=\bar{q}_{1} \gamma_{\mu}\left(1-\gamma_{5}\right) q_{2}$, and $a_{2}$ are the effective Wilson coefficients indicating color suppression. Because of the heavy $\Lambda_{c}^{+}$mass, the available phase space of $\Lambda_{c}^{+} \bar{\Lambda}_{c}^{-} K^{-}$is really small which result in the branching fraction $\sim \mathcal{O}\left(10^{-8}\right)$. This is not consistent with the current Belle result [30], shown in Table 1.5. So, $\Lambda_{c}^{+} \bar{\Lambda}_{c}^{-}$pair is unlikely to be produced dominantly through the direct three-body decay process. Cheng et al's paper[33] obtained a rule consistent with the Bell result by assuming the decay proceeds via formation of intermediate charmonium states in $\Lambda_{c}^{+} \bar{\Lambda}_{c}^{-}$. Later on, they also suggested that Final State Interaction( FSI) [34] may play an important role on the three-body decay. In the FSI process, the final state hadrons interact strongly after they are produced, as shown in Fig 1.6. In general, the FSI effect is only a next leading factor and negligible, and this is the reason that "the color transparency argument" is applicable for factorization calculation. In general, the FSI effect is very difficult to calculate, because it is too small and entangle with other effect. If the three-body decay is proved experimentally to be FSI dominant process, the FSI effect would be measurable. It would then be very useful in an accurate extraction of the 
direct $\mathrm{CP}$ violation.

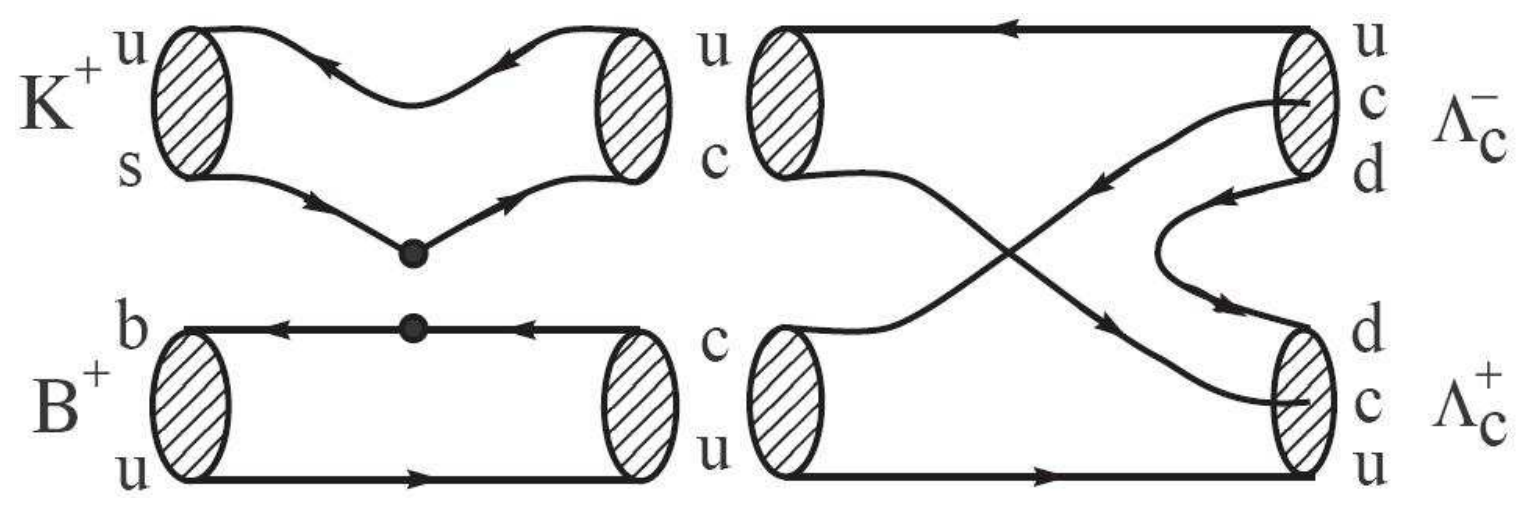

Figure 1.6: The flavor diagram of $B^{-} \rightarrow \Lambda_{c}^{+} \bar{\Lambda}_{c}^{-} K^{-}$which is arisen via $B^{0} \rightarrow D^{0} D^{0} K^{-}$

In this analysis, The $B$ decays listed in Table 1.6 are study by using run 14 data collected with the $B A B A R$ detector. A back-of-the-envelope estimate of the expected yield of the two-body $B^{-} \rightarrow \Xi_{c}^{0} \bar{\Lambda}_{c}^{-}$can be made from the total number of $B \bar{B}$ pairs $\left(\sim 228 \times 10^{6}\right.$ produced in Run $\left.1-4\right)$, a theoretical estimate of the $\Xi_{c}^{0} \rightarrow \Xi^{-} \pi^{+}$branching fraction $\mathcal{B}\left(\Xi_{c}^{0} \rightarrow \Xi^{-} \pi^{+}\right) \sim 2 \%$, and an approximate efficiency (4\%). Hence, we can have $\sim 6$ events reconstructed where we assume that $\mathcal{B}\left(B^{-} \rightarrow\right.$ $\left.\Xi_{c}^{0} \bar{\Lambda}_{c}^{-}\right) \sim 0.1 \%[28]$. However, the uncertainties in the branching fractions make this only an order of magnitude assessment of the expected yield.

\subsection{An overview of the thesis}

This thesis describes the exclusive measurements of the branching fractions of $B$ meson decays into charmed baryon pairs. The current chapter presents an overview 
Table 1.6: List of decay channels studied

\begin{tabular}{l}
\hline \hline Summarizes of the final states considered \\
\hline$B^{-} \rightarrow \Lambda_{c}^{+} \bar{\Lambda}_{c}^{-} K^{-}$ \\
$\Lambda_{c}^{+} \rightarrow p^{+} K^{-} \pi^{+}, \bar{\Lambda}_{c}^{-} \rightarrow p^{-} K^{+} \pi^{-}$ \\
$\Lambda_{c}^{+} \rightarrow p^{+} K^{-} \pi^{+}, \bar{\Lambda}_{c}^{-} \rightarrow p^{-} K_{S}^{0}, K_{S}^{0} \rightarrow \pi^{+} \pi^{-}$ \\
$\Lambda_{c}^{+} \rightarrow p^{+} K^{-} \pi^{+}, \bar{\Lambda}_{c}^{-} \rightarrow \bar{\Lambda}^{0} \pi^{-}, \Lambda^{0} \rightarrow p^{+} \pi^{-}$ \\
$\Lambda_{c}^{+} \rightarrow p^{+} K_{S}^{0}, \bar{\Lambda}_{c}^{-} \rightarrow p^{-} K^{+} \pi^{-}$ \\
$\Lambda_{c}^{+} \rightarrow \Lambda^{0} \pi^{+}, \bar{\Lambda}_{c}^{-} \rightarrow p^{-} K^{+} \pi^{-}$ \\
\hline $\bar{B}^{0} \rightarrow \Lambda_{c}^{+} \bar{\Lambda}_{c}^{-} K_{s}^{0}$ \\
$\Lambda_{c}^{+} \rightarrow p^{+} K^{-} \pi^{+}, \bar{\Lambda}_{c}^{-} \rightarrow p^{-} K^{+} \pi^{-}$ \\
$\bar{B}^{0} \rightarrow \Xi_{c}^{+} \bar{\Lambda}_{c}^{-}$ \\
$\Xi_{c}^{+} \rightarrow \Xi^{-} \pi^{+} \pi^{+}, \bar{\Lambda}_{c}^{-} \rightarrow p^{-} K^{+} \pi^{-}, \Xi^{-} \rightarrow \Lambda^{0} \pi^{-}$ \\
\hline$B^{-} \rightarrow \Xi_{c}^{0} \bar{\Lambda}_{c}^{-}$ \\
$\Xi_{c}^{0} \rightarrow \Xi^{-} \pi^{+}, \bar{\Lambda}_{c}^{-} \rightarrow p^{-} K^{+} \pi^{-}$ \\
$\Xi_{c}^{0} \rightarrow \Lambda^{0} K^{-} \pi^{+}, \bar{\Lambda}_{c}^{-} \rightarrow p^{-} K^{+} \pi^{-}$ \\
\hline \hline
\end{tabular}

of the theoretical background and a summary of previous measurements.

The $B$ factory facility is described in Chapter two, which was used to collect the data for the analysis. Chapter three gives an introduction to the BABAR trigger. The section for Trigger upgrade is not directly tied to this analysis, but is critical to the data collection; and the author contributed substantially to the effort. The last several chapters present the Physics analyses which include the data sample, signal selection, background studies, maximum likelihood fits, and finally the interpretation of the results. 


\section{CHAPTER 2 \\ EXPERIMENTAL FACILITY - BABAR DETECTOR AND PEP-II}

The B-Factory facility, PEP-II storage ring with the BABAR Detector, is located at the Stanford Linear Accelerator Center (SLAC). This experiment was designed to measure the $\mathrm{CP}$ violation in the neutral $\mathrm{B}$ meson decay into states which are also CP eigenstates[15], but in addition, provides an excellent laboratory for important measurements for a broad range of physics in the heavy quark and heavy lepton sectors. In addition to new discoveries, the very high luminosity helps make precision measurements in many areas as well.

\subsection{PEP-II accelerator}

The PEP-II accelerator, an upgrade of PEP(Position-Electron Project)[35], is an asymmetric $e^{+} e^{-}$collider with two storage rings shown in Fig 2.1: a high energy ring (HER) circulating electrons at $9 \mathrm{GeV}$, and a low-energy ring (LER) circulating positrons at $3.1 \mathrm{GeV}$, with PEP-II operating at a center-of-mass(CM) energy of 10.58 GeV. The HER is renovated from the original PEP storage ring, with the LER on top built expressly for the BABAR experiment. The energy asymmetry gives the CM a Lorentz boost in the lab frame with a $\beta \gamma=0.56$; this allows the $B$ mesons to travel a measurable distance from the production point to the decay point in the detector, and thus the time dependence of the $B$ pair's decay rates can be measured.

The $\Upsilon$ mesons are the ${ }^{3} S_{1}$ bound states of the $b \bar{b}$, with an angular momentum of one; the quark and anti-quark pairs are aligned to generate spin 1 with zero orbital 


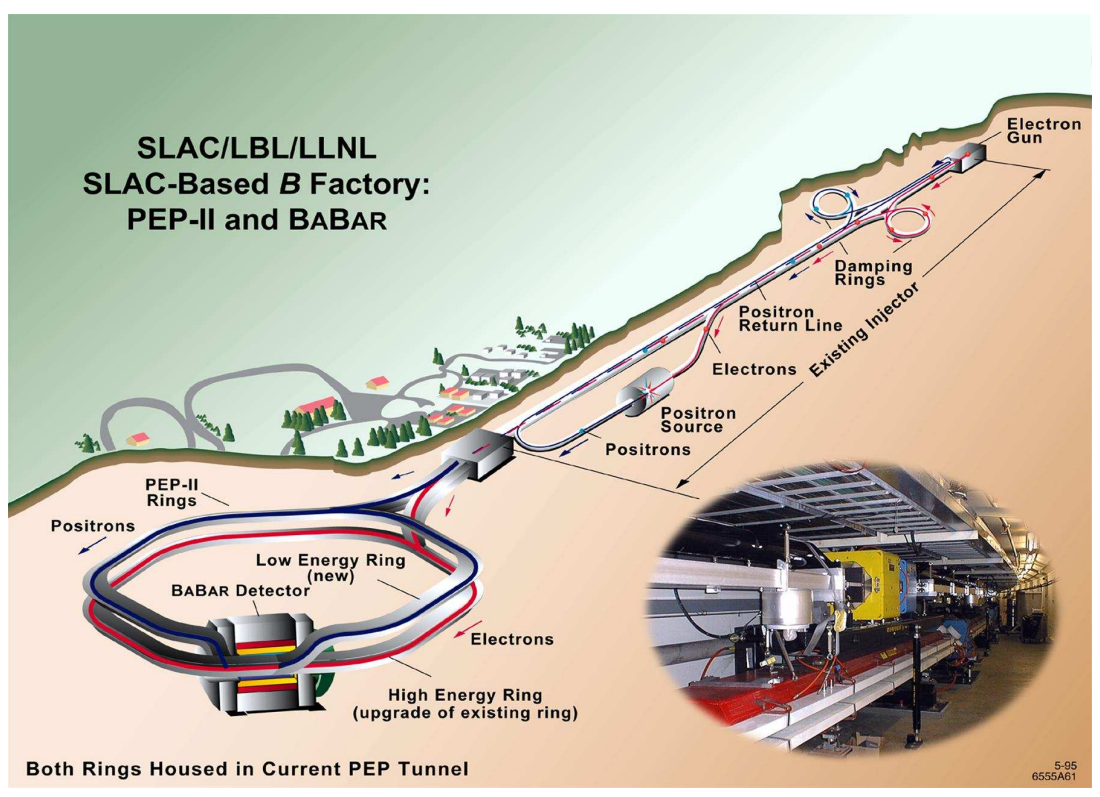

Figure 2.1: The schematic view of PEP-II.

angular momentum between them. The $\Upsilon(4 S)$ meson (Fig 2.2), with a mass of $10.58 \mathrm{GeV}$, is just above the threshold for production of a $B \bar{B}$ pair $(10.56 \mathrm{GeV})$, and decays to $B \bar{B}$ pairs $96 \%$ of the time.

PEP-II operating at a CM energy of of $10.58 \mathrm{GeV}$, optimized for $B \bar{B}$ production, is called a $B$ factory. The cross-sections of the selected $e^{+} e^{-}$processes at $\sqrt{s}=10.58$ are shown in Table 2.1. The ratio of $\sigma_{b \bar{b}}$ and $\sigma_{q \bar{q}}$ is about $1: 3$.

The electrons and positrons are generated and accelerated to their collision energies in the linac, and then transported through the bypass line, diverted into LER and HER by a powerful dipole magnet (kicker magnet). The beams in PEP-II are distributed in bunches of about $1 \mathrm{~cm}$ long in $z$, each bunch separated in time by 4.2ns. A total of 1658 bunches out of 3492 possible RF buckets are filled, with an 


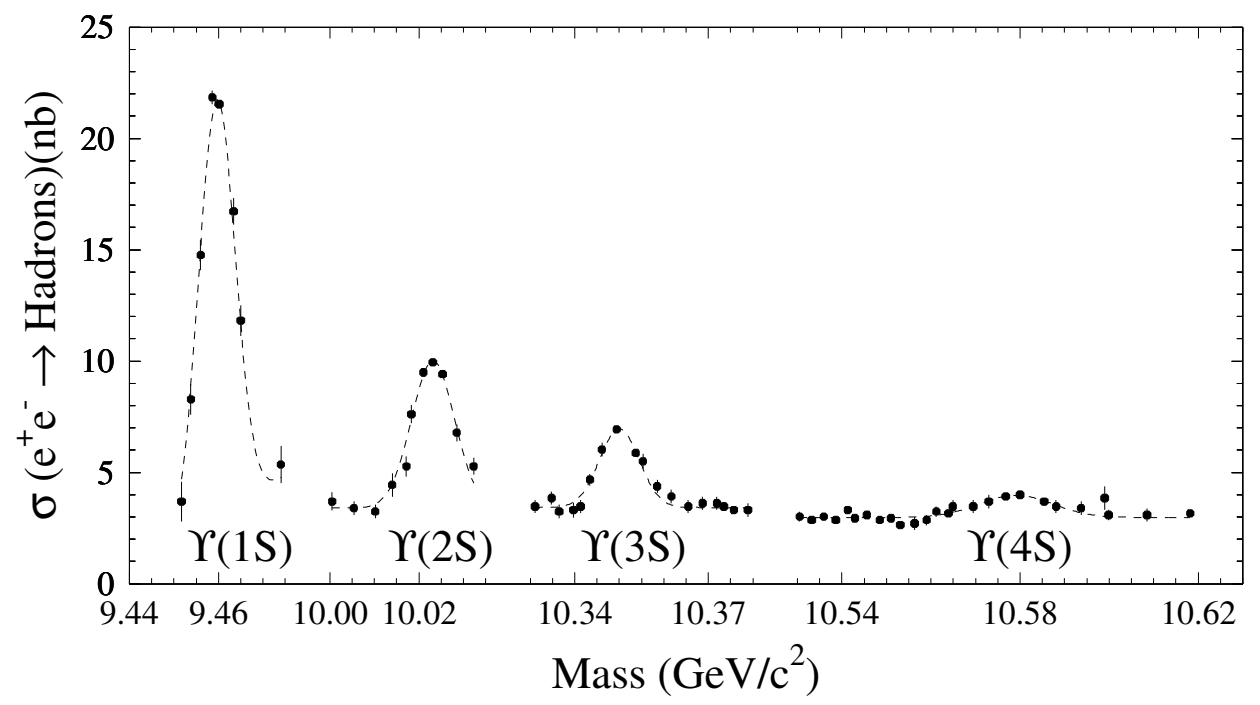

Figure 2.2: The cross-section of $e^{+} e^{-} \rightarrow$ hadrons at the $\Upsilon$ mesons (1S-4S) and in the continuum measured by the CLEO collaboration[36].

Table 2.1: Cross-sections, production and trigger rates for the principle processes at $\sqrt{s}=M(\Upsilon(4 S))$

\begin{tabular}{cccc}
\hline \hline Mode & Cross-section (nb) & Production rate $(\mathrm{Hz})$ & L1 rate $(\mathrm{Hz})$ \\
\hline$b \bar{b}$ & 1.10 & 3.2 & 3.2 \\
\hline$c \bar{c}$ & 1.30 & 10.2 & 10.1 \\
$u \bar{u}, d \bar{d}, s \bar{s}$ & 2.197 & 159 & 156 \\
\hline$e^{+} e^{-}$ & $\sim 53$ & 3.5 & 3.1 \\
$\mu \bar{\mu}$ & 1.16 & 2.8 & 2.4 \\
$\tau^{+} \tau^{-}$ & 0.94 & & \\
\hline \hline
\end{tabular}

electron and a positron bunch containing about $2.1 \times 10^{10}$, and $5.9 \times 10^{10}$ particles, respectively. The electron and the positron bunches collide head-on at the interaction region, shown in Fig 2.3. The collision axis is off-set from the $z$-axis of the BABAR 
detector by about 20 mrad in the horizontal plane. After each collision, the beams are separated in the $y z$-plane by a pair of dipole magnets located at $\pm 21 \mathrm{~cm}$ on each side of the interaction point (IP) and a series of quadrupole magnets for focusing the beams.

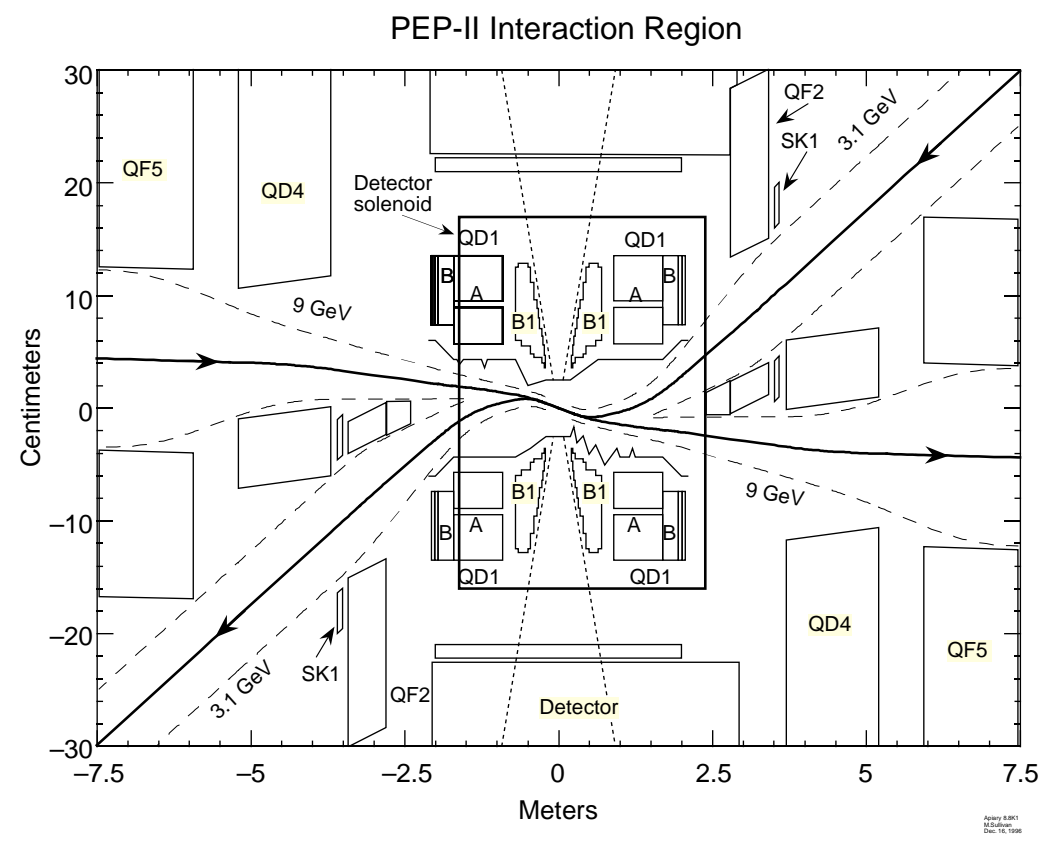

Figure 2.3: The schematic of interaction region.

The yardstick of the performance of an accelerator is the luminosity (L) it delivers; this is defined as

$$
L=f \cdot n \frac{N_{1} N_{2}}{A}
$$

where $N_{1}$ and $N_{2}$ are the number of particles in each bunch, $\mathrm{n}$ is the number of 
bunches in either beam, and $\mathrm{f}$ is the RF frequency. Generally speaking, the number of $\Upsilon(4 S)$ produced depend directly on the luminosity. Even though PEP-II was designed for a luminosity of $3 \times 10^{33} \mathrm{~cm}^{-2} \mathrm{~s}^{-1}$, it has now achieved a peak Luminosity of $12.069 \times 10^{33} \mathrm{~cm}^{-2} \mathrm{~s}^{-1}$ with 1722 bunches with currents of $2900 \mathrm{~mA}$ and $1875 \mathrm{~mA}$ in HER and LER, recently, on Aug, 2006. Since 1999, the luminosity delivered by PEP-II to date is $431 \mathrm{fb}^{-1}$ of which $B A B A R$ has recorded $412 \mathrm{fb}^{-1}$, which represents an efficiency of more than $95 \%$.

\section{$2.2 \quad B A B A R$ detector}

The BABAR detector[37] is a nearly hermetic multiple layer detector situated at the PEP-II accelerator optimized for studies of the of the energy range of the B decays with capabilities for reconstruction of heavy quark decay vertices (around $\left.1 \times 10^{-6}\right)$. Figure 2.4 shows the longitudinal view of $B A B A R$ detector, composed of five sub-detectors to detect both charged and neutral particles.

- The charged tracks are detected and their momentum measured by the tracking system consisting of

- a silicon vertex tracker (SVT),

- a drift chamber (DCH), (vertexing is also performed by the tracking system, along with some particle identification at low momentum);

- detector of internally reflected Cherenkov radiation (DIRC) is used primarily for charged-hadron identification; 
- A finely segmented electromagnetic calorimeter (EMC) is used to detect photons and electron identification along with neutral pions; it also helps with muon detection;

- The instrumented flux return (IFR): as the name implies, this is made of steel and is the return path of the magnetic flux of the superconducting magnet located just outside the EMC, enveloping the rest of the sub-detectors inside. It is interleaved with Resistive Plate Chambers (RPC) which help detect muons and neutral hadrons.

The superconducting solenoidal magnet produces a 1.5-T magnetic field which bends the trajectories of the charged particles, the curvature of the track leading to a momentum measurement. Only the IFR is outside the magnetic field.

\subsubsection{BABAR coordinate system}

The BABAR detector uses a right-handed coordinate system with the $\mathrm{z}$ axis defined as the principle axis of the drift chamber, but offset by 20 mrad relative to the beam axis. The $+z$ direction is defined as the direction of the electron beam with the $+\mathrm{y}$ axis pointing upward, and the $+\mathrm{x}$ axis horizontal pointing away from the PEP-II storage ring. The polar angle $\theta$ and the azimuthal angle $\phi$ are defined with respect to the $+\mathrm{z}$ direction, and with respect to the $+\mathrm{x}$ direction in the $\mathrm{x}-\mathrm{y}$ plane, respectively. 


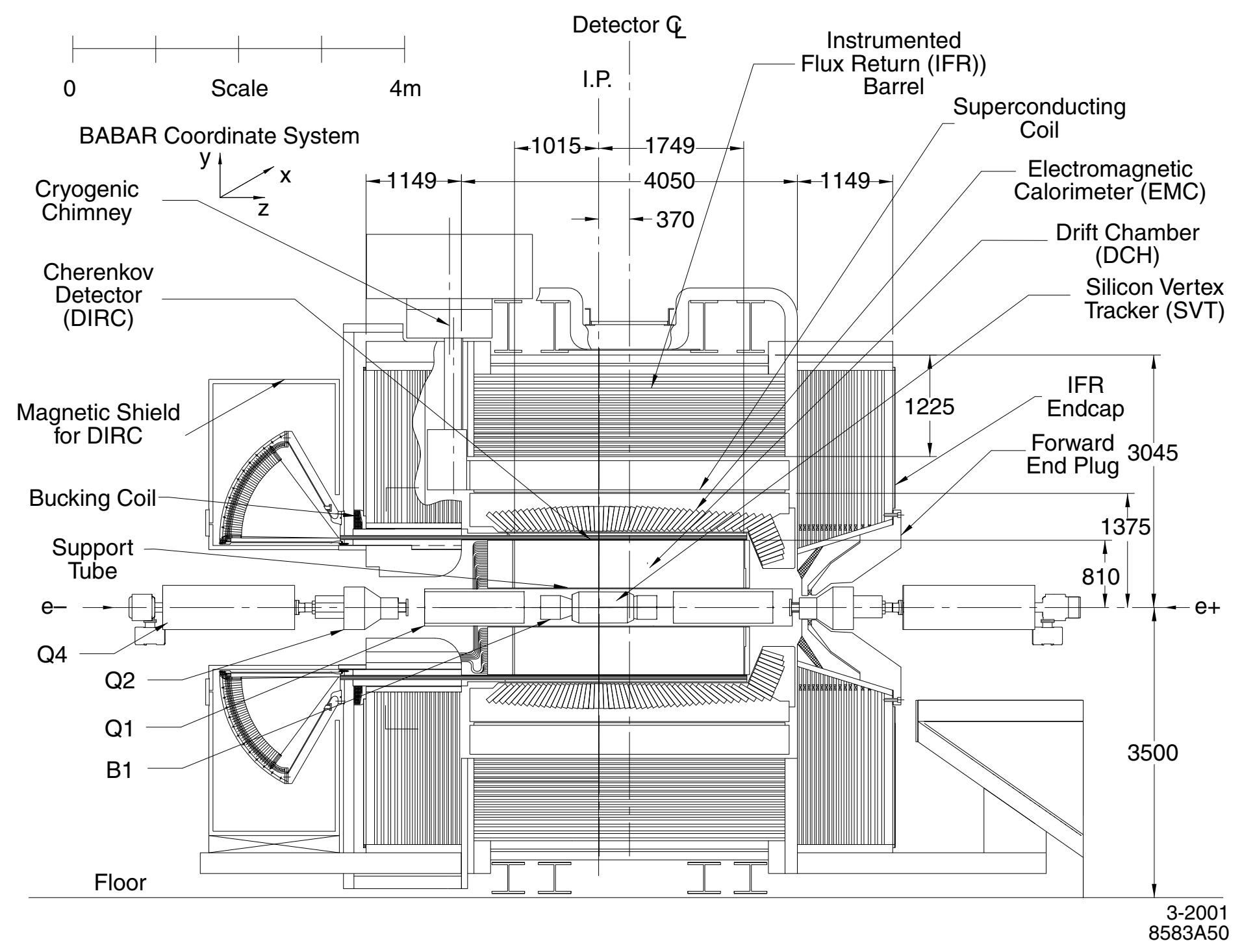

Figure 2.4: The longitudinal section of the detector. The dimension is in $\mathrm{mm}$. 


\subsection{Tracking system}

Tracking system is composed of low density material. Charged particles ionize the traversing medium (silicon in SVT, gas in DCH) along their trajectory through the detectors, the ionization are collected and recorded by the detectors and are later used in reconstruction of the track. The rate of energy loss $(-\mathrm{dE} / \mathrm{dx})$ by the charges particle measured over its trajectory also provide information about the mass of the particle, thereby providing a particle id.

\subsubsection{Silicon vertex detector}

The Silicon Vertex Tracker (SVT) is the innermost detector component of $B A B A R$. It is designed to provide precise reconstruction of charged particle trajectories and decay vertices near the interaction region which is critical for the measurement of the time dependence of the B-decays for measuring the CP asymmetry. The SVT provides standalone tracking for particles with a transverse momentum less than $120 \mathrm{MeV} / c$, below which the DCH alone can not provide a reliable measurement. The SVT also provides the best measurement of a track angle required to achieve the resolution for DIRC for high momentum tracks.

The SVT is made of five double-sided layers of silicon strip sensors, shown in figure 2.5. It provides, up to ten hits (spatial points) on the track of each charged particle. By extrapolating these points backwards towards the interaction point, it is possible to obtain an accurate measurement of the the direction of the charged particle leading to the $\mathrm{z}$ position of the $B$ meson decay vertex. 


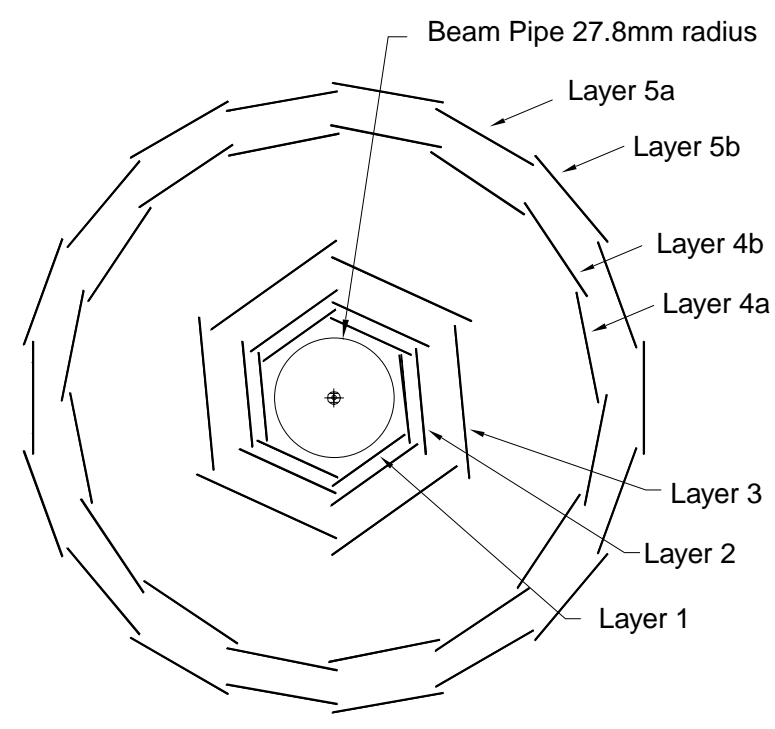

Figure 2.5: The transverse section of SVT.

The strips on the inner side of each layer are oriented perpendicular to the beam direction to measure the $\mathrm{z}$ coordinate, and the ones on the outer side of each layer are orthogonal to the $\mathrm{z}$ trips to measure the $\phi$ coordinate. Each of the first three inner layers has six modules which are straight and close to the beam pipe. The design minimizes the impact of multiple scattering in extrapolation of the track to the vertex, and achieves an excellent impact parameter measurement (the position and the angle information of the decay vertex). The fourth and fifth layers have sixteen and eighteen modules, respectively, which are arranged in arch-shaped to increase the solid angle coverage.

The angular coverage is extended down to $350 \mathrm{mrad}\left(20^{\circ}\right)$ in polar angle in the forward direction, and $520 \mathrm{mrad}\left(30^{\circ}\right)$ in the backward direction, as shown in Fig 2.6. The spatial resolution is $10-15 \mu \mathrm{m}$ in the three inner layers, and $40 \mu \mathrm{m}$ in 
the two outer layers.

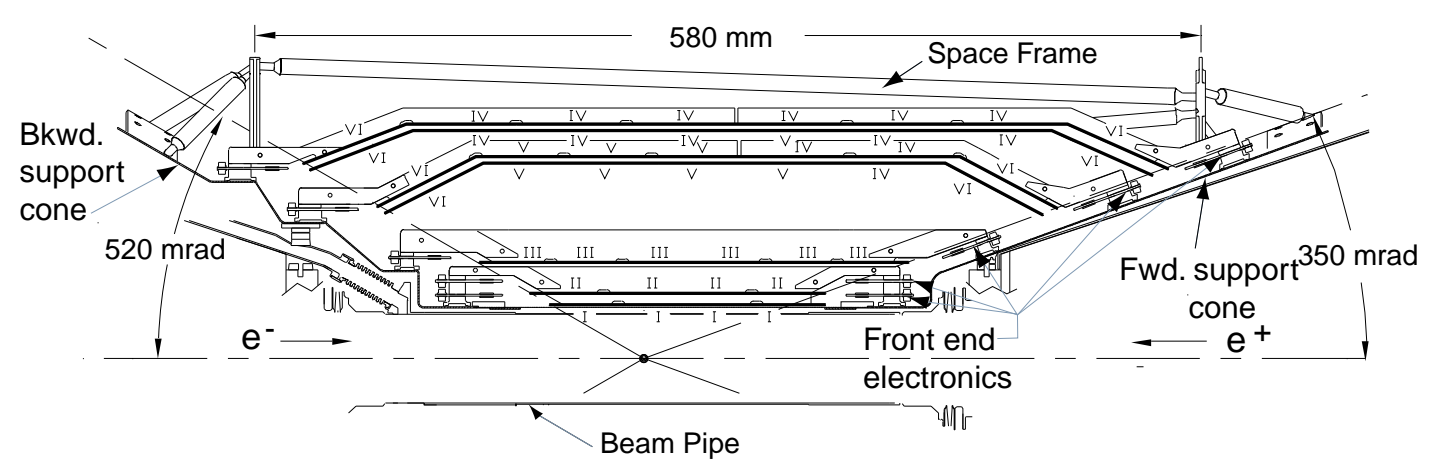

Figure 2.6: The longitudinal section of SVT. The roman numbers label the six different types of sensors.

The hit information from a total of approximately 150,000 readout modules are fanned-out to ATOM 9 (A Time-Over-Threshold Machine) front-end Integrated chips. In the ATOM chips, the signals are digitized and buffered. The digitized signals then are converted into optical signals and sent out to DAQ for further processing.

The SVT has worked extremely well with both $z$ and $\phi$ measurements efficiencies at $97 \%$. The hit resolutions for $z$ and $\phi$ vary between 10 and $40 \mu m$ depending the track incident angle. The resolution in the $d E / d x$ measurement is about $14 \%$. 


\subsubsection{Drift chamber (DCH)}

The Drift chamber is the principle device for charged track reconstruction. It provides reliable pattern recognition leading to good track reconstructions with precise momentum measurements, as well as reconstruction of the decay vertices for long-lived particles like $\Lambda^{0}$, which might decay outside the SVT. Furthermore, the $\mathrm{DCH}$ also aids in particle identification by precisely measuring the ionization $\operatorname{loss}(\mathrm{d} E / \mathrm{d} x)$, especially for low momentum particles and the particles traversing in the extremely forward and backward direction, complementing to the sub-detector DIRC .

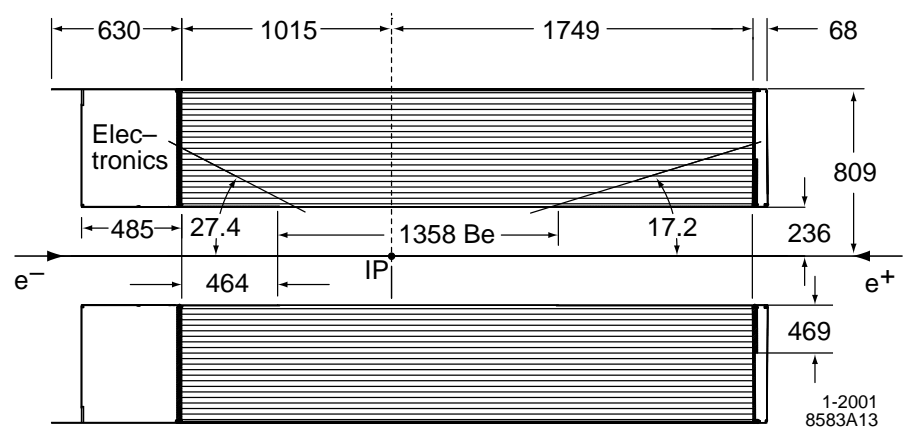

Figure 2.7: The side view of drift chamber. The dimension is in mm. The interaction point is offset from the center of DCH.

The DCH is comprised of a $2.8 \mathrm{~m}$ long cylindrical volume bound by a pair of aluminum endplate, with inner and outer radii $0.236 \mathrm{~m}$ and $0.809 \mathrm{~m}$, respectively. The central axis of the cylinder approximately coincides with the axis of the $1.5 \mathrm{~T}$ 
magnetic field. Fig 2.7 shows the longitudinal cross-section of the DCH. The polar angle coverage is $17.2^{\circ}$ in the forward direction and $152.6^{\circ}$ in the backward direction.

The DCH is composed of 7104 densely packed hexagonal drift cells. In each cell, a gold-plated tungsten-rhenium sense wire with a radius of $20 \mu \mathrm{m}$ is placed at the center, and Au-plated Aluminum field wires with radii of 80 or $120 \mu \mathrm{m}$ are placed at the boundary. The sense wire provides the high voltage with the field wires grounded, and the potential difference between the sense and field wires is typically 1960 Volts. The DCH contains 40 cylindrical layers so that each charged particle can obtain up to 40 spatial and ionization measurements. The layers are grouped by fours into 10 superlayers, shown in figure 2.8; the stereo angles of the superlayers alternate between axial (A) and stereo (U and V) pairs, in a pattern AUVAUVAUVA. The cell axes of the axial superlayers are parallel to the z-axis, and the U,V layers have a small angles (between $\pm 45 \mathrm{mrad}$ and $\pm 76 \mathrm{mrad}$ ) with respect to the z-axis, allowing measurements of longitudinal as well as axial position information. The drift chambers uses a gas mixture of helium and isobutane in a $80: 20$ ratio by volume which helps minimize multiple-scattering (because of the long radiation decay length).

The basic idea of a drift chamber is to measure the trajectory of a charged particle by collecting electrons from the ionization left in its wake. When a charged particle passes through the Drift cell, it ionizes the gas along its path. The electrons drift liberated in the primary ionization drift toward a sense wire in the high field. The accelerated electrons give rise to secondary ionizations in the gas which also accelerate eventually causing an avalanche. This leads to an amplification of the ionization signal 


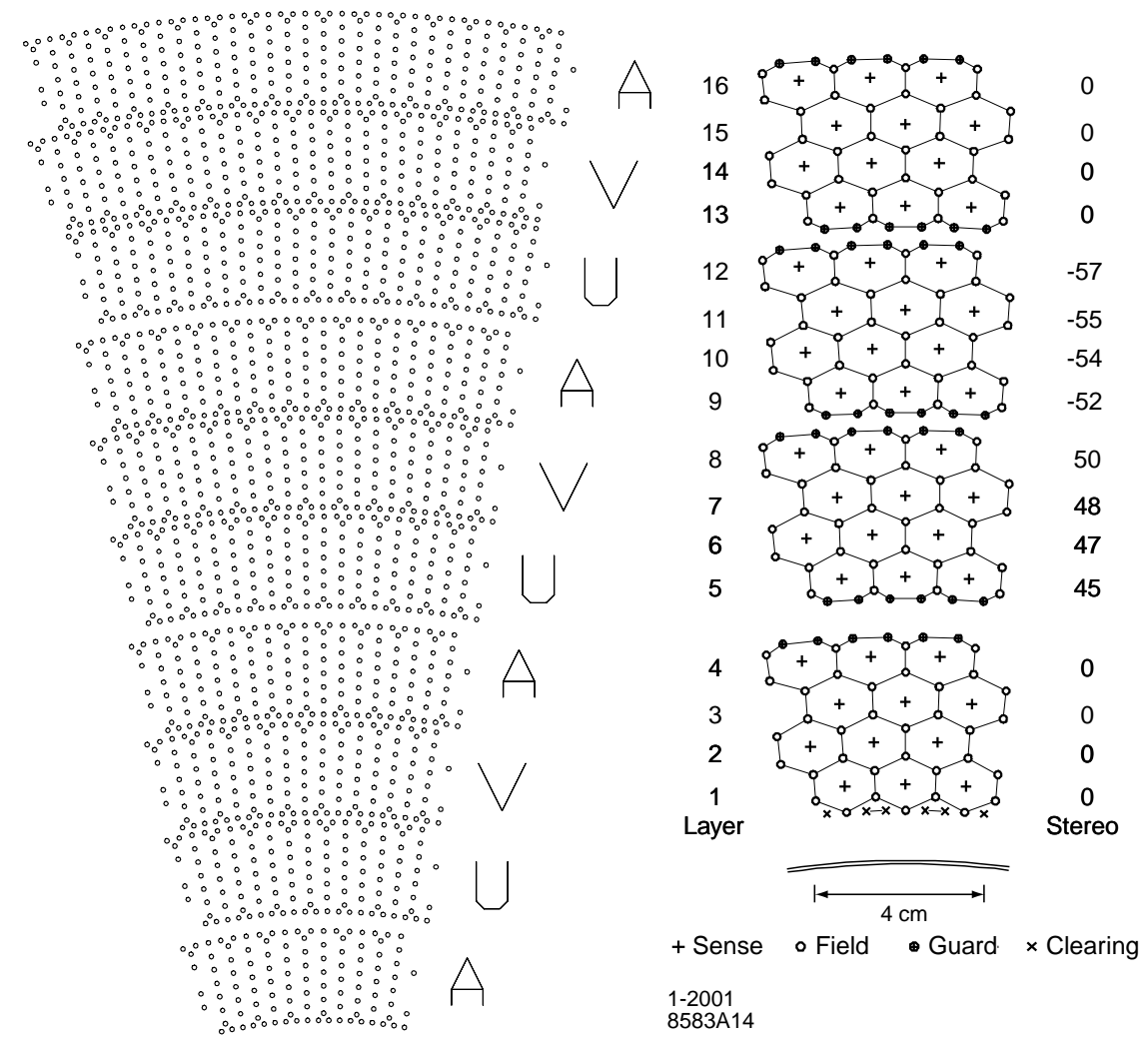

Figure 2.8: The transverse view of the drift chamber. (a) The transverse view of the wires in the drift chamber. (b) Schematic layout of the drift cell in the first four superlayers. The dimension of the numbers on the right side in mrad.

received by the anode - sense wire. The drift velocity of electrons is $22 \mu \mathrm{m} / \mathrm{ns}$, and the distance between the ionizing track and sense wire is determined from the drift time which is accurately measured. The momentum of a charged particle is measured from the curvature of its trajectory in the magnetic field; the radius of curvature determines $p_{T}$ (momentum transverse to B field). Mutually perpendicular E and B fields cause charged particles to drift in the Ex B direction. The Lorentz Angle, defined as the angle between the drift velocity and the E field, for a $1.5 \mathrm{~T}$ field, for 
the Babar DCH is about 32 degrees. The specific energy loss for charged particles traversing the DCH is derived from the total charge deposited in each drift cell.

The performance of the tracking system has been studied with cosmic ray muons, lepton pairs and multi-hadrons events. By comparing the number of tracks detected in the SVT and extrapolating into the DCH to those actually reconstructed by the $\mathrm{DCH}$, the average tracking efficiency of $\mathrm{DCH}$ has been determined to be $98 \pm 1 \%$ per track for momenta greater than $200 \mathrm{MeV} / c$ and $\theta>500$ mrad at the design voltage of $1960 \mathrm{~V}$. A charged track is described by five parameters: $d_{0}$ and $z_{0}$ (transverse distance and $\mathrm{z}$ coordinate at the point of closest approach of the helical track to the $\mathrm{z}$ axis), $\phi_{0}$ (azimuthal angle), $\lambda$ (dip angle with respect to the transverse plane), and $1 / p_{t}$ (track curvature). The cosmic ray tracks in the upper and lower halves of

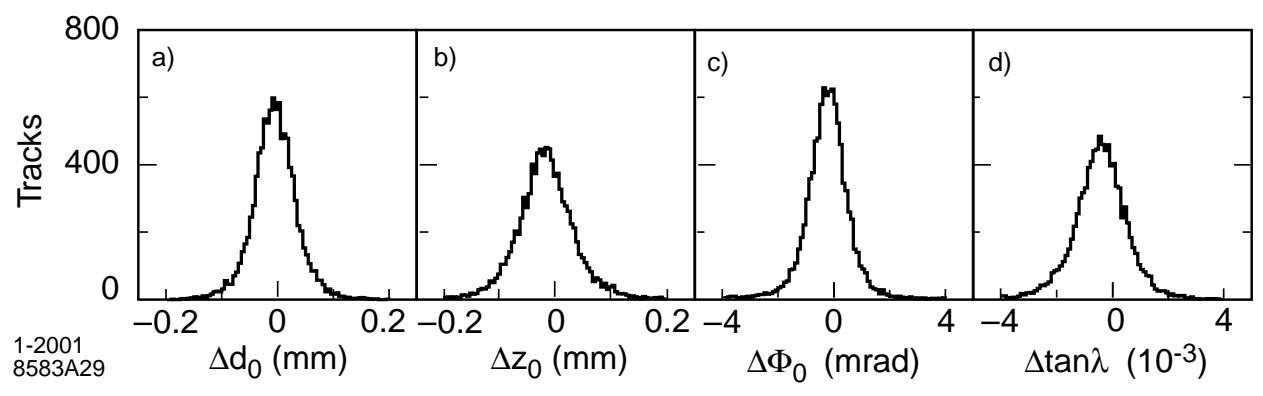

Figure 2.9: The differences of four tracking parameters.

detector are measured separately, and the differences in their track parameters, shown 
in Fig 2.9, are used to measure the resolution.

$$
\begin{aligned}
\sigma_{d_{0}} & =23 \mu \mathrm{m} \quad \sigma_{\phi_{0}}=0.4 \mathrm{mrad} \\
\sigma_{z_{0}} & =29 \mu \mathrm{m} \quad \sigma_{\tan \lambda}=0.53 \cdot 10^{-3}
\end{aligned}
$$

The resolution in $p_{t}$ measured from the cosmic ray events, are:

$$
\sigma_{p_{t}} / p_{t}=\left((0.13 \pm 0.01) \cdot p_{t}+(0.45 \pm 0.03)\right) \%
$$

where $p_{t}$ is measured in $\mathrm{GeV} / c$.

The truncated mean of the energy loss $(\mathrm{dE} / \mathrm{dx})$ per track in $\mathrm{DCH}$, measured as a function of the track momentum, are illustrated in Figure 2.10. The measured $\mathrm{dE} / \mathrm{dx}$ resolution for Bhabha events is typically $7.5 \%$.

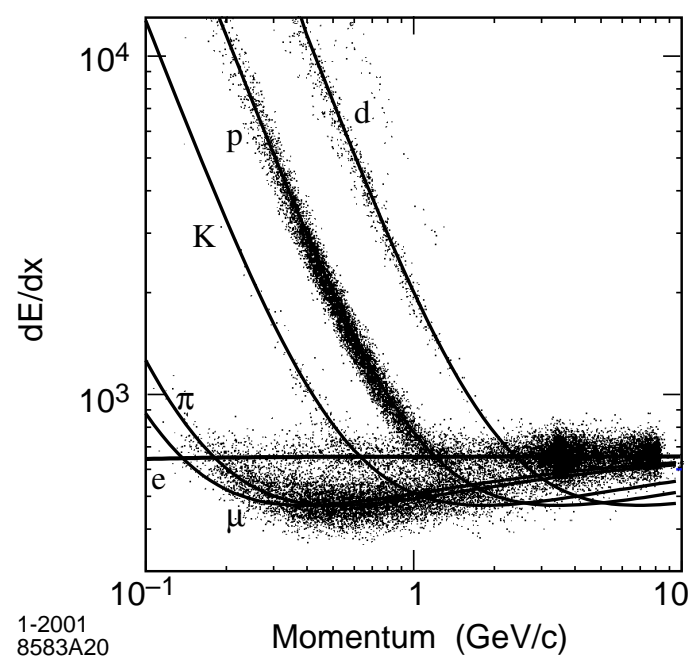

Figure 2.10: The measured $d E / d x$ in the drift chamber as a function of track momentum with superimposed curves derived from the Bethe-Bloch prediction. 


\subsection{Detector for internally reflected cherenkov light}

Detector of Internal-reflected Cherenkov light (DIRC) is a novel ring-imaging Cherenkov radiation detector, designed to provide information of the particle identification. It is capable of separating pions from kaons with a more than $\sim 4 \sigma$ discrimination from $0.7 \mathrm{GeV}$ up to $4.5 \mathrm{GeV} / c$. It is azimuthally symmetric w.r.t the $z$-axis with an azimuthal coverage of about $92 \%$; the polar angle coverage is expanded from $25.5^{\circ}$ in the forward direction to $141.4^{\circ}$ in the backward direction.

The DIRC operates on detecting the Cerenkov radiation by a traversing particle, the light emitted when a charged particle passes through a transparent medium at a speed greater than the speed of the light in that medium. The velocity of a particle is defined as $\beta=p / E$ relativistically. For a material with refractive index $\mathrm{n}$ $(n \sim 1.473$ at $B A B A R)$, the condition for emission of Cherenkov radiation is $\beta>1 / n$. The Cherenkov photons are emitted at an angle $\left(\cos \theta_{c}=1 / \beta n\right)$ to the direction of the charged particle, in a cone around the momentum vector of the particle. By combining the measurement of the Cerenkov angle with the measurement of the momentum of the particle, it is possible to obtain the mass, and therefore the identity of the particle. The principle of the light production, transport and imaging in the DIRC is illustrated in Figure 2.11.

The transparent medium in DIRC consists of 144 quartz bars, nearly $5 \mathrm{~m}$ long, mounted inside twelve sealed aluminum containers, called bar boxes. Each bar box contains twelve quartz bars. The Cerenkov radiation emitted by the charged particle while traversing the quartz bar undergoes total internal reflection along the length of 


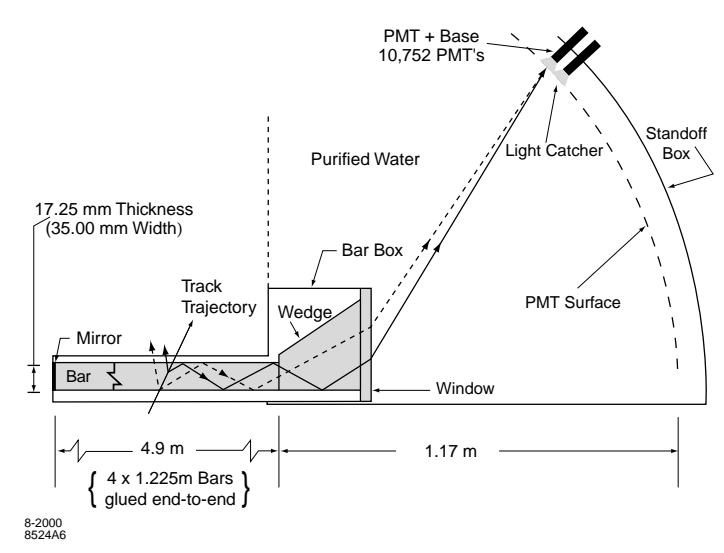

Figure 2.11: A view of the DIRC system.

the bar, a process which preserves the Cerenkov angle information. Mounted behind the rear IFR door is a tank of about 6000 liters of purified water containing 10752 photomultiplier tubes. The refractive index of the water is very close to that of the quartz, so the total internal reflection at the silica-water interface is minimized.. The cone of Cherenkov light emerges from the ends of the quartz bars and is projected onto this array of photomultiplier tubes, allowing the Cherenkov angle to be measured. The backward end of each bar has a small trapezoidal piece of quartz glued to it. This allows for a recovery of those photons that would otherwise be lost at the surface. The layouts of DIRC are shown in Figure 2.12 and Figure 2.13.

The measured single photon resolution in the DIRC is $1.7 \mathrm{~ns}$, close to the expected value of the PMT resolution, 1.5 ns. Figure 2.14 shows the variation of Cherenkov angle vs the momenta of the tracks, calculated at the entrance of the track to the bar box. The average kaon selection efficiency is $(96.2 \pm 0.2) \%$, and the 


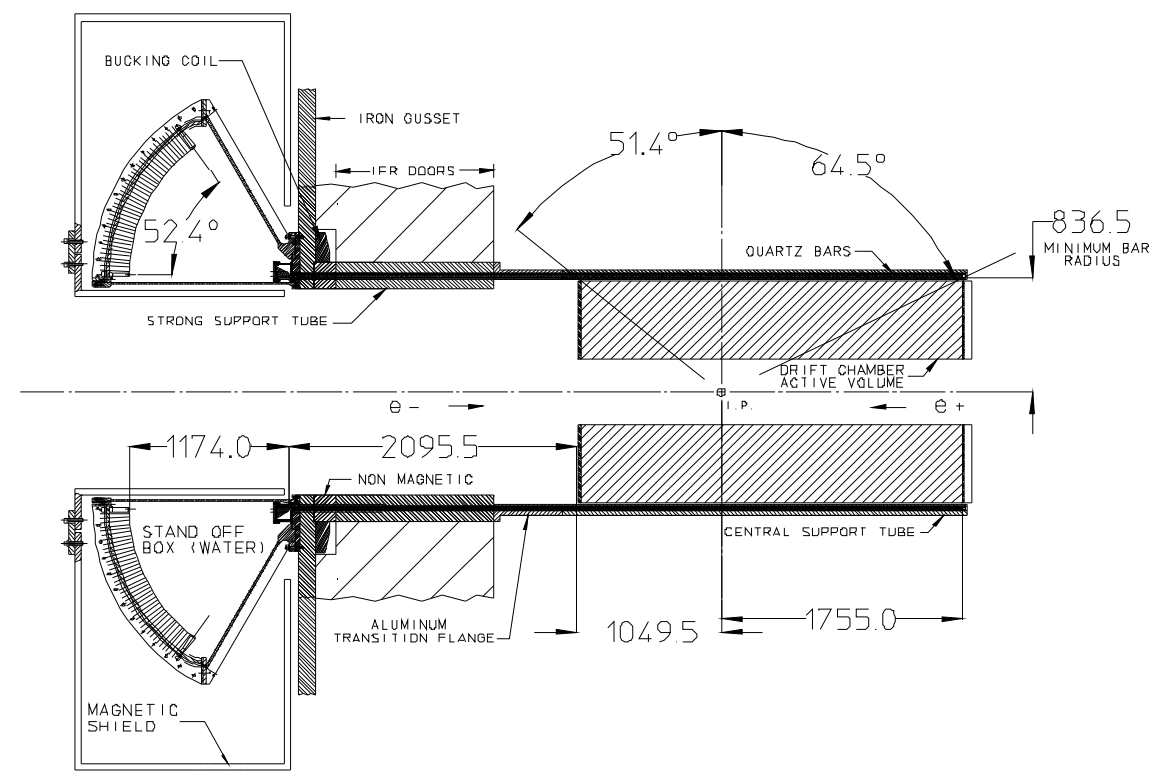

Figure 2.12: The elevated view of the nominal DIRC system geometry. All the dimensions are in $\mathrm{mm}$.

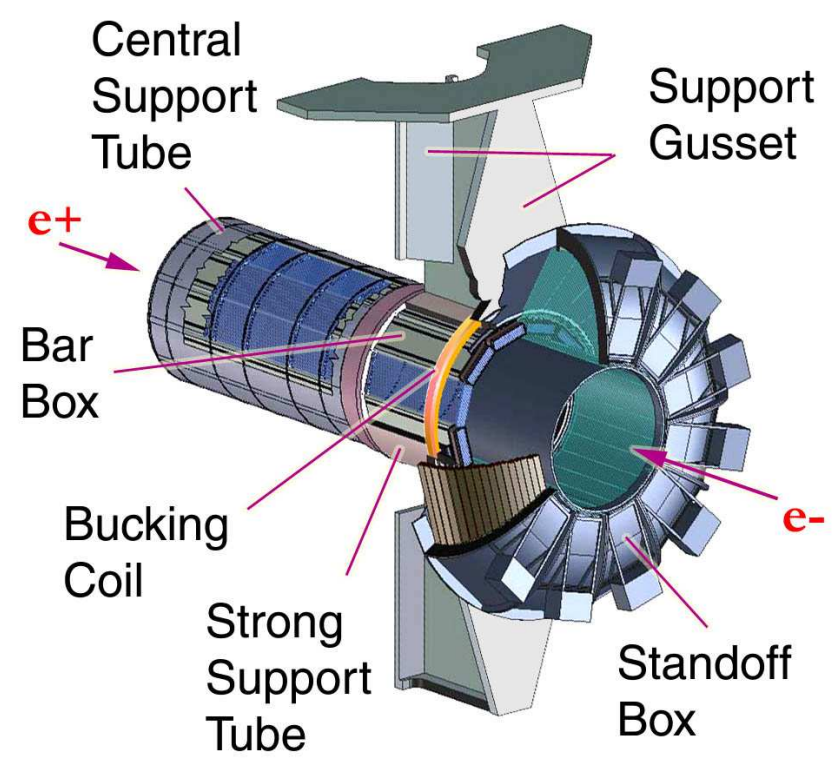

Figure 2.13: A view of the DIRC system. 
pion mis-identification rate is $(2.1 \pm 0.1) \%$.

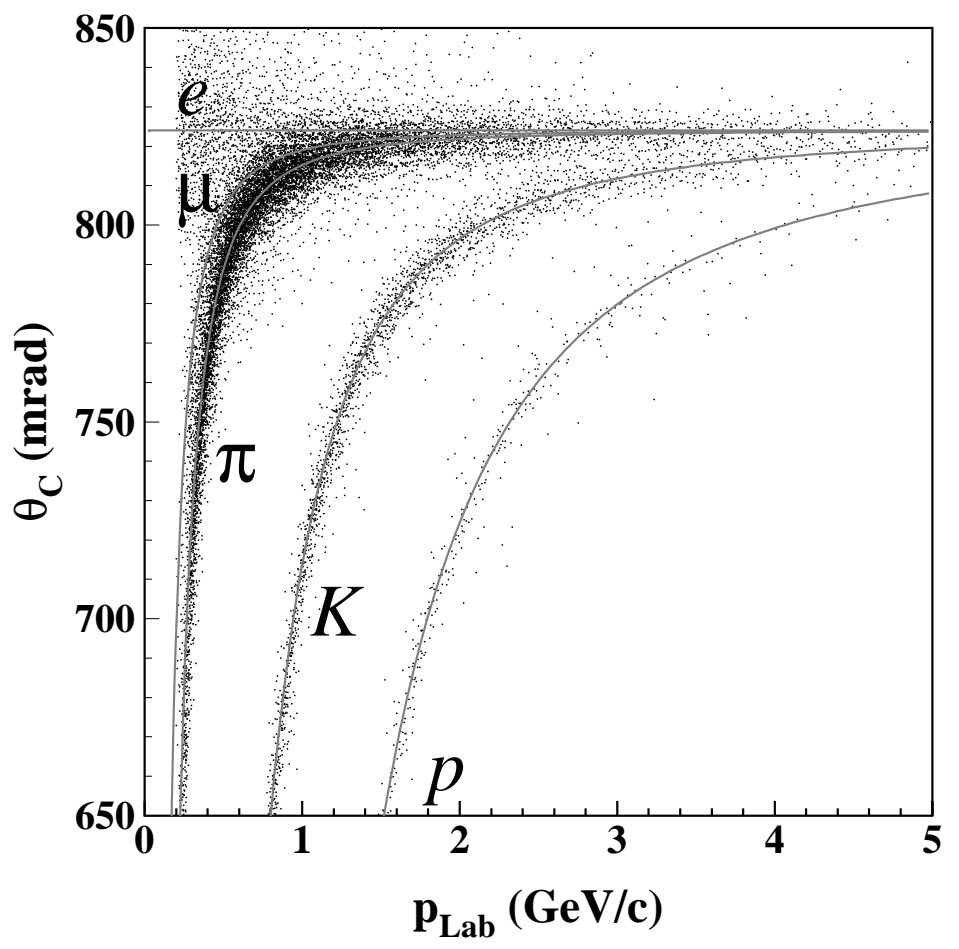

Figure 2.14: The Cherenkov angle of tracks distribution plotted according the momentum of the tracks. The grey lines are the predicted values.

\subsection{Electromagnetic calorimeter (EMC)}

The electromagnetic calorimeter (EMC) is designed to provide energy and angular measurement for photons over the energy range between $20 \mathrm{MeV}$ and $9 \mathrm{GeV}$, AND ELECTRONS by measuring electromagnetic showers with excellent efficiency. Many decay processes involve $\pi^{0}, \eta \mathrm{s}$, which decay into low energy photons. Electro- 
magnetic and radiative processes, such as $e^{+} e^{-} \rightarrow \gamma \gamma$ and $e^{+} e^{-} \rightarrow \gamma D \bar{D}$, include photons with high energies. The EMC is critical in measuring these processes. In addition, it helps separate electrons from charged hadrons by observing: (1) the spatial distribution of the energy deposit in the calorimeter, and (2) the ratio of the shower energy to the track momentum in the tracking devices.

The EMC is a crystal calorimeter (Figure 2.15), made of a total of 6580 crystals of Thallium doped Caesium Iodide (CsI(TL)), a scintillating material. The reasons to use $\mathrm{CsI}(\mathrm{Tl})$ is because it has a small Molière radius and a high light yield which help achieve good position resolution. Each crystal is about $29.6-32.4 \mathrm{~cm}$ in length and about $22.1-36.6 \mathrm{~cm}^{2}$ in cross-section from front to back. The EMC is divided into the barrel and the forward endcap sections. There are 5760 crystals in the barrel region, arranged in 48 rows in $\theta$, each row with 120 identical crystals symmetrically in $\phi$. The polar angular coverage of the barrel in the lab frame is $-0.80<\cos \theta_{\text {lab }}<0.89$. The 820 endcap crystals are piled up in a conic shape tilted at $22.7^{\circ}$ angle with respect to the vertical direction. The twenty modules are arranged in eight rings in $\theta$ (41 crystals in $\phi)$. The endcap covers the region of $-0.89<\cos \theta_{l a b}<0.97$. Because of the asymmetric nature of the beam energies, no backward endcap is used.

Photons, electrons and positrons produce electromagnetic showers in the EMC and the resulting scintillation light is detected by photo-diodes mounted at the back of the crystals. The amount of the light produced depends on the energy of the particle. In addition to photon identification, electrons can be discriminated from pions by the way the energy deposited is shared between neighboring crystals. 


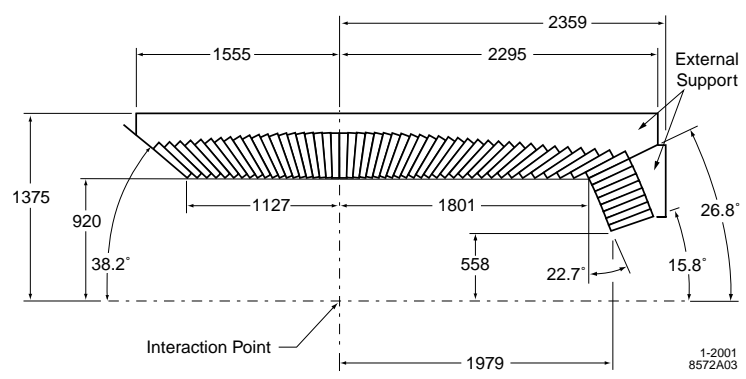

Figure 2.15: The longitudinal view of the top half of the EMC. All the dimensions are in $\mathrm{mm}$.

The energy resolution is described empirically in terms of two terms added in quadrature

$$
\frac{\delta_{E}}{E}=\frac{(2.32 \pm 0.30) \%}{\sqrt[4]{E(\mathrm{GeV})}} \oplus(1.85 \pm 0.12) \%
$$

The first term is primarily due to the statistical fluctuations in the scintillation photon yield, electronic noise and beam-generated background. The second term is a constant, dominant at high energies. It results from the non-uniformity in light collection, leakage and absorption in front of and between the crystals. The Angular resolution is measured from measuring $\pi^{0}$ and $\eta$ decays to two photons, derived from a fit to an empirical parameterization in energy dependence as

$$
\delta_{\theta}=\delta \phi=\left(\frac{3.87 \pm 0.07}{\sqrt{E(\mathrm{GeV})}} \pm 0.00 \pm 0.03\right) \mathrm{mrad}
$$

\subsection{Detector for muons and neutral hadrons - IFR}

Surrounding the superconducting solenoid is the Instrumented Flux return (IFR), the outermost of BABARsub-detectors. It is designed primarily to detect the passage of muons, which are highly penetrating particles. Also, the IFR is used to 
detect any long lived neutral particles which pass through the EMC, such as the $K_{L}^{0}$.

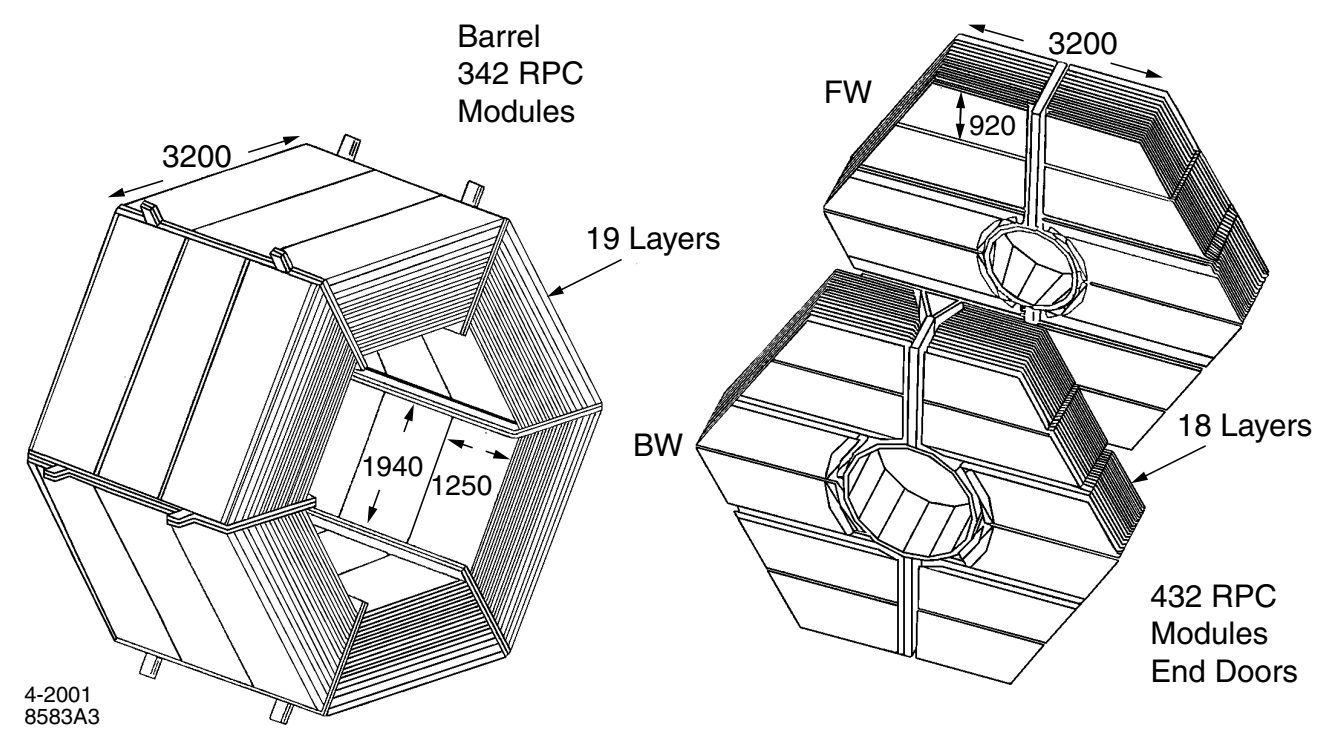

Figure 2.16: IFR structure: barrel sector, forward endcap and backward endcap.

The IFR uses the steel flux return of the magnet as the hadron absorber allowing only the muons to pass. The steel is segmented in 18 plates, increasing in thickness from $2 \mathrm{~cm}$ for inner nine plates to $10 \mathrm{~cm}$ for the outermost plates. The single gap resistive plate chambers (RPCs), are installed in the gaps of the finely segmented steel. There are $57 \mathrm{RPC}$ modules in each of the six sextant barrel sectors, 108 modules in each of the four half (NOT CLEAR) end doors, as illustrated in Fig. 2.16. In addition, $32 \mathrm{RPC}$ modules are installed in the two cylindrical layers between the EMC and the magnet cryostat to detect particles exiting the EMC outwards. So, a 
total of 806 RPC modules cover the active area about $2000 \mathrm{~m}^{2}$.

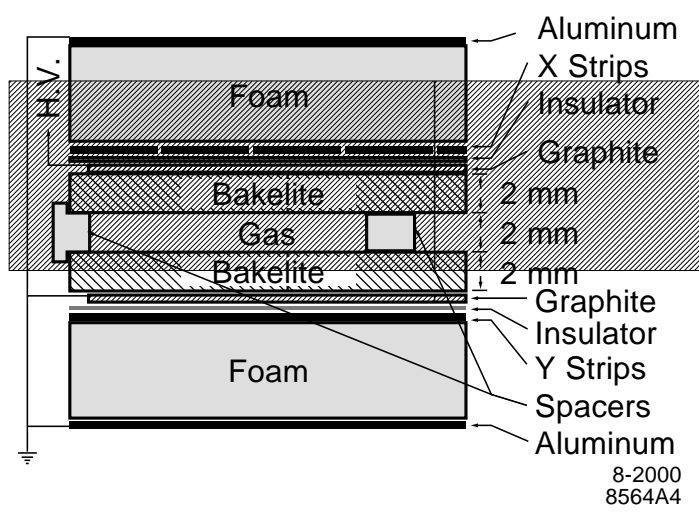

Figure 2.17: IFR structure: barrel sector, forward endcap and backward endcap.

Each of the planar RPCs, shown in Fig 2.17, consists of two Bakelite sheets, whose external surfaces are coated with graphite to achieve the desired surface resistivity. One of the graphite sheet is connected to a $8 \mathrm{kV}$ high voltage, the other to the ground, and protected by an insulating mylar film. The gap between the two sheets is $2 \mathrm{~mm}$, and is filled with non-flammable gas mixture, typically $56.7 \%$ argon, $38.8 \%$ freon $134 \mathrm{a}$, and $4.5 \%$ isobutane. The RPCs detect streamer from ionizing particles via capacitative aluminum strips on both sides of the gaps.

The initial performance of the RPCs resulted in a detection efficiency of $90 \%$ in the momentum range of $1.5<p<3.0 \mathrm{GeV} / c$, from $\mu \mu$ ee final state events. The $K_{L}^{0}$ detection efficiency is between $20 \%$ and $40 \%$ in the momentum range of 1 and $4 \mathrm{GeV} / \mathrm{c}$. However, over time the efficiency of the RPCs became lower than $10 \%$, and 
many of the RPC modules died. Hence, they have been replaced by Limited Streamer Tubes (LST) [38] in the summer of 2006. 


\section{CHAPTER 3 \\ TRIGGER SYSTEM AND TRIGGER UPGRADE}

\subsection{Trigger system overview}

Trigger system is one of the critical components of the BABAR Data Acquisition System. The Trigger is a real-time filtering process to select interesting physics events in the detector while rejecting the high rate background with a high, stable and measurable efficiency. The trigger system has a two level hierarchy: the Level 1 (L1) trigger resides in hardware, and Level 3 (L3) trigger is in software primarily.

The overview of the BABAR trigger and DAQ system layout is shown in Figure 3.1. At PEP-II the $e^{+} e^{-}$collisions take place with a $4.2 \mathrm{~ns}$ bunch crossing;

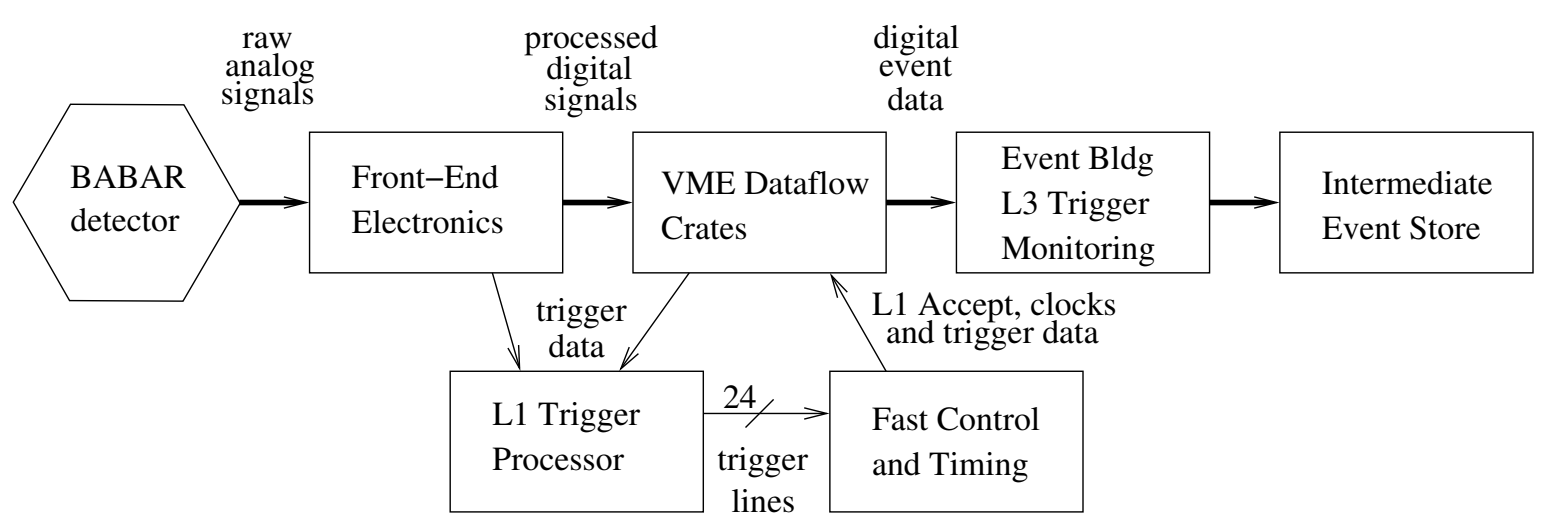

Figure 3.1: The BABAR electronics, trigger, DAQ system

essentially almost continuously. At this rate, the Front End Electronics (FEE) for all detector subsystems (except the SVT) continuously digitize the detector data and 
buffer them with a $12.9 \mu$ s deep buffer. The dedicated L1 system must deliver the L1 trigger to the Fast Control and Timing System (FCTS) within this latency period. Upon receipt of an L1 Accept (L1A) signal, all detector data are read out by the dataflow system, when feature extraction (e.g., energy, momentum) is performed and transported to the online processing farm as input to the L3. A typical L1 trigger rate is less than 2kHz: the maximum capacity of the BABAR data acquisition(DAQ) for L1 output rate. The L3 performs a further rate reduction categorizing the events into various main physics classes, at an output L3 rate of typically $120 \mathrm{~Hz}$; this satisfies the physics requirements, as illustrated in Table 2.1, which lists the production rate of interesting physics processes.

\subsection{Level 1 trigger}

The BABAR L1 trigger trigger system uses fast signals from the detectors to recognize occurrence of potentially interesting physics events, on which it issues a L1 Accept signal. L1 trigger system is composed of four subsystems: the Drift Chamber Trigger (DCT), the Calorimeter Trigger (EMT), the Instrumented Flux Return Trigger (IFT), and Global Level Trigger (GLT). The SVT is not used for input in L1, as the background rate is too high. The L1 trigger decision is based on charged tracks in the DCH, showers in the EMC and tracks detected in the IFR. Most physics events are recognized both by DCT and by the EMT, and thereby provide a high degree redundancy, while the IFT plays a supplementary role, mostly for diagnostic purpose. The outputs from the three systems are combined in the global trigger (GLT) unit, 
which issues an L1-Accept signal to the detector.

\subsubsection{L1 DCH trigger}

The DCT uses information from the DCH electronics to trigger events with charged tracks. the DCT system consists of three types of modules: Track Segment Finder (TSF) modules, the Binary Link Tracker (BLT) modules, and the Transverse Momentum $\left(p_{t}\right)$ Discriminators (PTD). All the DCT modules perform the control and algorithmic functions in multiple FPGAs. The L1 hardware is housed in five 9U VME crates. The signal transmission between these modules, DCH, and GLT are handled by a $6 \mathrm{U}$ back-of-crate interface board behind each main board.

\subsubsection{TSF}

The TSF modules use DCH wire hit patterns to find track segment information which is used in later stages. The bit-wide discriminator outputs from the 7104 cells of the DCH are transmitted to the TSF modules via 24 fiber-optic GLINKs (gigabit links) in frames of 316 bits at a rate of $4 \mathrm{MHz}$. One GLINK pattern is assigned to one TSF board. There are two types of TSF boards: 16 TSFx boards receive $300 \times 16$ bits from superlayers 1-4 and 8-10 in 1/16 wedges of the DCH; 8 TSFy boards/modules receive $288 \times 8$ bits data from superlayers $5-7$ in an $1 / 8$ wedge as shown in Fig 3.2. The track segment finding is organized in pivot cells. Physically, pivot cells are the cells in the third layers of each superlayer, there are a total of 1776 pivot cells. Each pivot cell and seven neighboring cells compose a pivot group. Some of the cells are shared with other pivot groups, but only one pivot cell is in one pivot group. In order to 


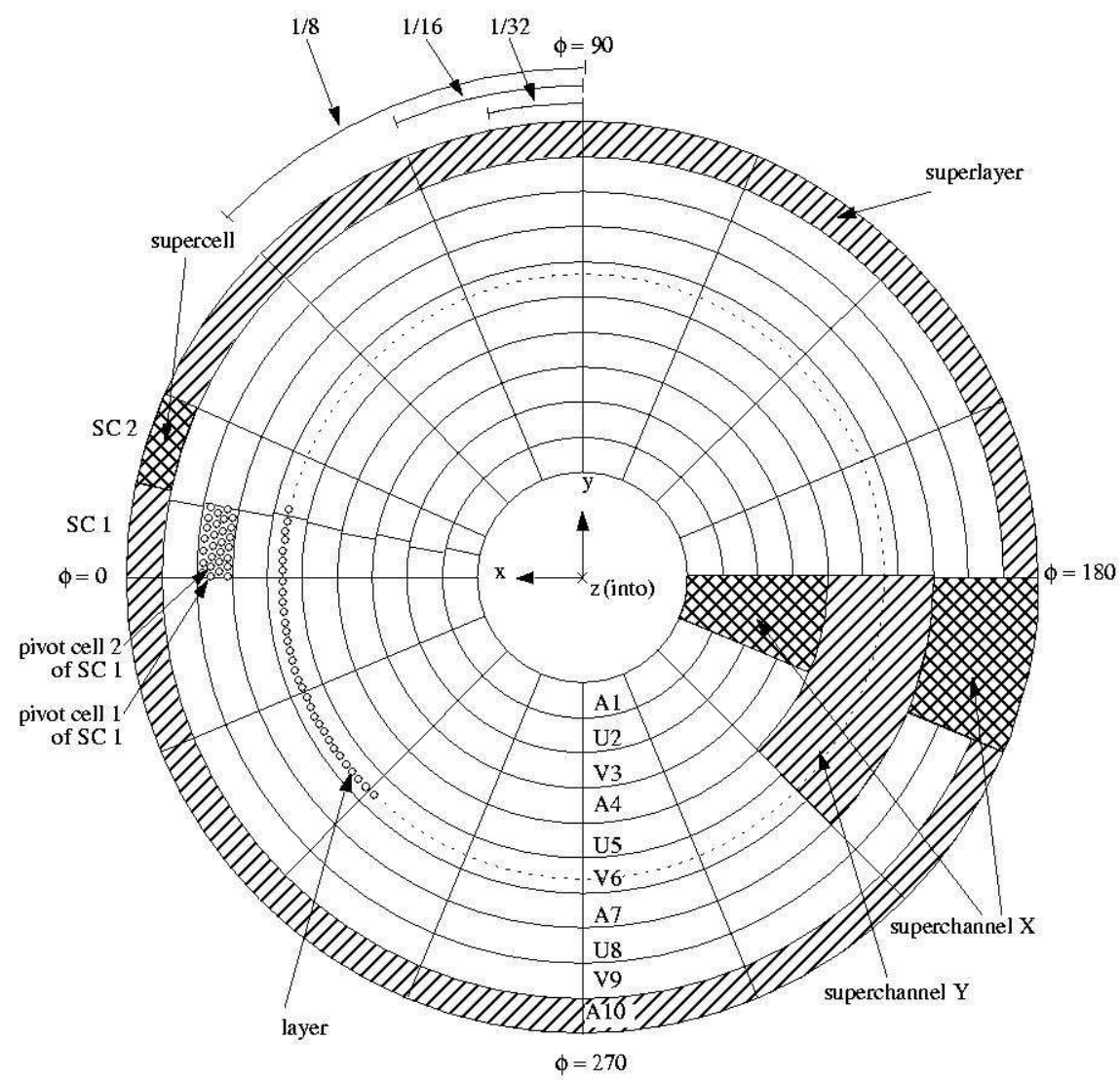

Figure 3.2: $\mathrm{X}-\mathrm{Y}$ view of the drift chamber, illustrating definition of terms.

complete the 8-cell pivot group, some hit information is shared between neighbouring TSF modules. Each cell is assigned a two-bit counter that is incremented at every clock tick for which a signal is present. In this way, the drift-time history for each cell is recorded. Typically the time for an ionization drifting to a sense wire is no more than 4 clock ticks. Each track segment finder engine of the TSF modules is associated with a pivot group and checks the eight cells of the group in every $269 \mathrm{~ns}$ with a predefined look-up table. If hits are found in three or four layers, and close in time, the track segment is consider as acceptable; which of three subsequent clock 
ticks produce the best pattern is decided, and the position and time information of the segments is recorded. The resolution on $\phi$ is about $1 \mathrm{~mm}$.

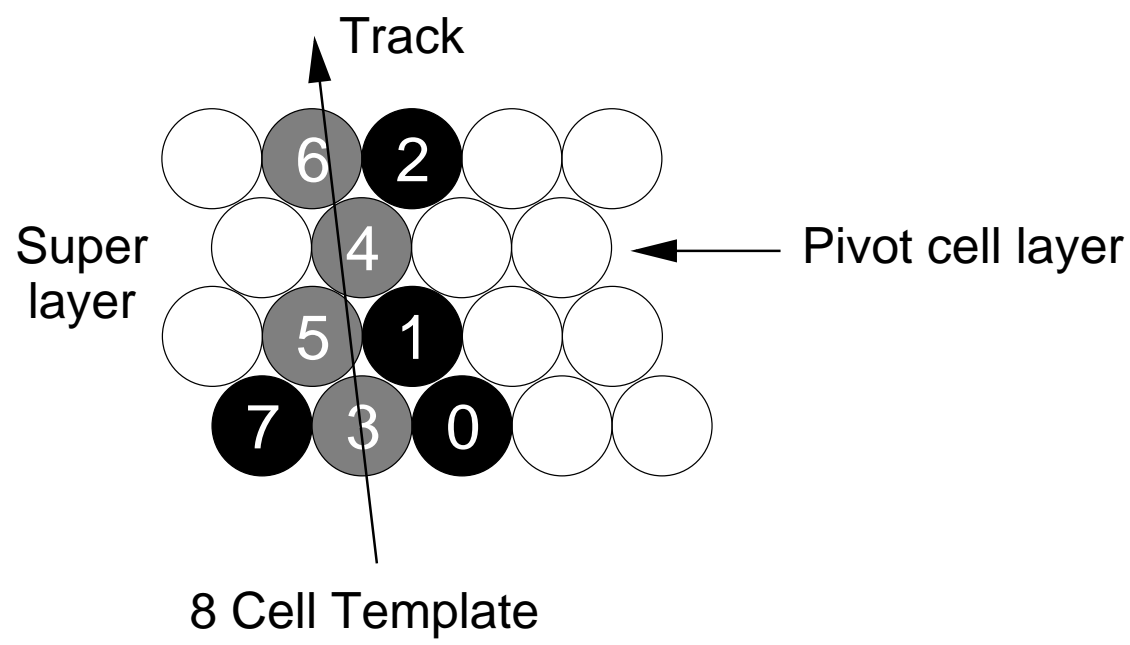

Figure 3.3: The represent of TSF pivot group. Cell 4 is called pivot cell, and cell 0-7 form a pivot group.

Finally, the coarse hit data - 320 1-bit segment hit signals from 24 TSF modules are output to the BLT module, and each bit represents whether a segment is found in a supercell ( $1 / 32$ of a superlayer). The fine segment information of a track in the axial layers(A1,A4,A7 and A10) is sent to the PTD every 269 ns. This data is also sent to the DAQ for quality assurance.

\subsubsection{PTD}

The PT discriminator (PTD) is used to process the $\phi$ information of high quality track segments to look for a track with $p_{t}$ greater than a predetermined value 
which is configurable. There are 8 PTD modules in the DCT system, and each PTD receives segment hit data from six TSF modules (four TSFx and two TSFy), which cover axial segments in 1/4 wedge of the DCH. By using segment hits in either superlayer A7 or A10 (first A10, second A7 superlayer) as "seeds", the searching algorithm is initiated by a PTD engine. An envelope for tracks above the minimum $p_{t}$ is defined using the IP, and a track segment position in one of the seed superlayers. If a seed if found, the engine searches for the segments in this envelope from the other three Axial superlayers by comparing it with a look-up table. The look-up table defines the limits for the position of each individual seed, the segment position for each of three other axial superlayers, and the effective $p_{t}$ threshold, set at $800 \mathrm{MeV} / c$.

If a segment is found in at least 2 of 3 remaining superlayers, the resulting hight- $p_{t}$ object, $A^{\prime}$ is reported with its supercell position in the unit of $1 / 32$ wedge of DCH. The efficiency of $A^{\prime}$ track is shown in Figure 3.4.

\subsubsection{BLT}

The Binary Link tracker (BLT) links the track segments from the TSF into complete tracks. There is only one BLT module in the DCT system, which handles all the 320 bits of data from the TSF modules every 134 ns. The BLT input data are combined using a logical OR with a programmable mask pattern. The mask forces the system takes into account the dead or highly ineffective cells to prevent inefficiency in tracking.

The track linker uses segment hits in the innermost superlayer A1 as seeds, 


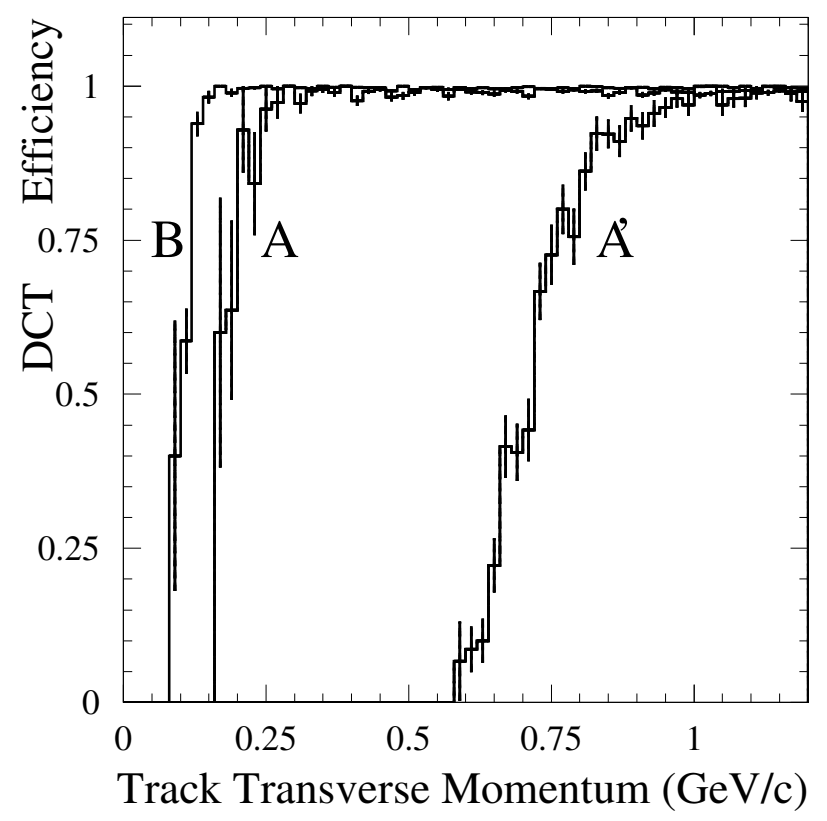

Figure 3.4: The DCT track efficiency versus momentum for A, B, and $A^{\prime}$ tracks.

and then searches for more hits tracking radially outwards. Due to inefficiencies in DCH, a real track may not generate a segment hit in all layers, and the coarse hits are allowed to miss segments in one or two superlayers. The segment hits in two consecutive superlayers are required to lie azimuthally within a certain range of the superlayers. The range is chosen such that it allows for track curvature in the magnetic field and dip angle variations taking the intrinsic geometry of the DCH into consideration. For transition between axial and stereo layers, the second segment should be in one of the three supercells closest to the first segment in $\phi$ position. For transition between two stereo layers, the range is limited within five supercells.

Hence, tracks that can reach superlayer 5 of the DCH is classified as a "B" 
track; while tracks that exit superlayer 10 is defined as an "A" track. The BLT module outputs two $\phi$ maps to GLT at a $7.44 \mathrm{MHz}$ rate, one for the A tracks and another for the B tracks. The resolution of hit information is reduced from 1/32 wedge to $1 / 16$ wedge by a 2 -to- 1 conversion. The efficiencies of $\mathrm{A}$ and $\mathrm{B}$ tracks are shown in Figure 3.4 .

\subsubsection{L1 EMC trigger}

The EMT continually processes signals from the EMC channels in condensed form into energy and cluster positions and sends the results to the L1 Global trigger. The EMT determines the time of the reconstructed particles/clusters. The continuous stream of data from the cluster sums represents multiple samples over the shaped analog signals from the physical energy deposits. These data determine the best estimate of the time of arrival of the physical particle/cluster. The trigger is based on the 40-fold azimuthal symmetry of the EMC. For trigger purposes, the EMC is divided into $7 \times 40(\theta \times \phi)$ towers. Each of the barrel's 240 towers consists of 24 crystals and each of the endcap tower contains 19-22 crystals. All the crystals in each $9^{\circ} \phi$ sector are summed and added in neighbouring pairs to identify energy deposits which span adjacent sectors (to form $40 \phi$ sums). This is therefore a one-dimensional $(\phi)$ projection of the two-dimensional $(\theta$ and $\phi)$ geometry.

The data which are sent to the GLT are the bits identifying the presence of the the trigger objects in each $\phi$ region in the form of M, G, E, X or Y trigger objects. The first three correspond to three different energy thresholds of the complete $\theta$ sums 
of the towers in the $\phi$ sector. The X bit is for MIP-like objects in the forward endcap only; while the $\mathrm{Y}$ bit is for Bhabha type events with energy deposits in the backward part of the barrel.

Table 3.1: Trigger primitives for the DCT and EMT

\begin{tabular}{lllcc}
\hline \hline & Description & Origin & No. of bits & Threshold \\
\hline B & Short track reaching DCH superlayer 5 & BLT & 16 & $120 \mathrm{MeV} / c$ \\
A & Long track reaching DCH superlayer 10 & BLT & 16 & $180 \mathrm{MeV} / c$ \\
$\mathrm{~A}^{\prime}$ & High $p_{T}$ track & PTD & 16 & $800 \mathrm{MeV} / c$ \\
\hline M & All- $\theta$ MIP energy & EMT & 20 & $100 \mathrm{MeV}$ \\
G & All- $\theta$ intermediate energy & EMT & 20 & $250 \mathrm{MeV}$ \\
E & All- $\theta$ high energy & EMT & 20 & $700 \mathrm{MeV}$ \\
X & Forward endcap MIP & EMT & 20 & $100 \mathrm{MeV}$ \\
Y & Backward barrel high energy & EMT & 10 & $1 \mathrm{GeV}$ \\
\hline \hline
\end{tabular}

Note: Most energy thresholds are adjustable; those listed are typical values.

\subsubsection{L1 IFR trigger}

The L1 IFR trigger (IFT) is used to identify $\mu^{+} \mu^{-}$and cosmic ray events. As mentioned before, the IFR is segmented into ten regions. In each region, 8 layers are selected for triggering. The signals from all $\phi$ strips in these layers are combined into a single bit with fast $O R$ logic, and a sector is declared active when at least 4 of the 8 layers have hits in a given 134 ns time interval. Finally, the signals from the 10 sectors are combined into 7 trigger primitives (objects), as defined in Table 3.2, encoded as a three-bit trigger word and sent to the GLT. 
Table 3.2: IFT trigger primitive

\begin{tabular}{ll}
\hline Object $(\mathrm{U})$ & Trigger condition \\
\hline 1 & $\geq 2$ muons, topologies other than $\mathrm{U}=5-7$ \\
2 & 1 muon in the backward endcap \\
3 & 1 muon in the forward endcap \\
4 & 1 muon in the barrel \\
5 & 2 back-back muons in the barrel and 1 forward \\
6 & 1 muon in the barrel and 1 forward \\
7 & 2 back-back muons in the barrel \\
\hline
\end{tabular}

\subsubsection{L1 global level trigger}

The Global level trigger receives nine trigger primitives from the DCT, EMT and IFT as listed in Table 3.1 and 3.2. From the input data, the GLT generates up to 24 different triggers, each based on a specific physics requirement. The triggers are passed along to the Fast Control System to make final trigger decision to be distributed to the $B A B A R$ readout systems.

As the input primitive signals are from difference sources, they arrive at GLT with different latencies ranging from $0.2 \mu \mathrm{s}$ to $7.2 \mu \mathrm{s}$. Configurable delays, as illustrated in Fig 3.5, are applied to them to align them in time; some signals are stretched to get enough overlap with other signals. Next, the trigger inputs from the DCT and the EMT are matched and combined, and $16 \phi$-map trigger objects are formed: A, B, Back-to-back objects $\left(A^{*}, B^{*}\right)$, high $p_{t}$ track object $A^{\prime}, \mathrm{M}, \mathrm{G}, \mathrm{E}, \mathrm{Y}$, large-angle pair of $\operatorname{clusters}\left(M^{*}, G^{*}\right)$, EM, track-cluster matching objects (BM, AM, $\left.A^{\prime} \mathrm{M}\right)$, BMX (MIP cluster with beam background veto) and one IFT object U. Finally, the 17 trigger 


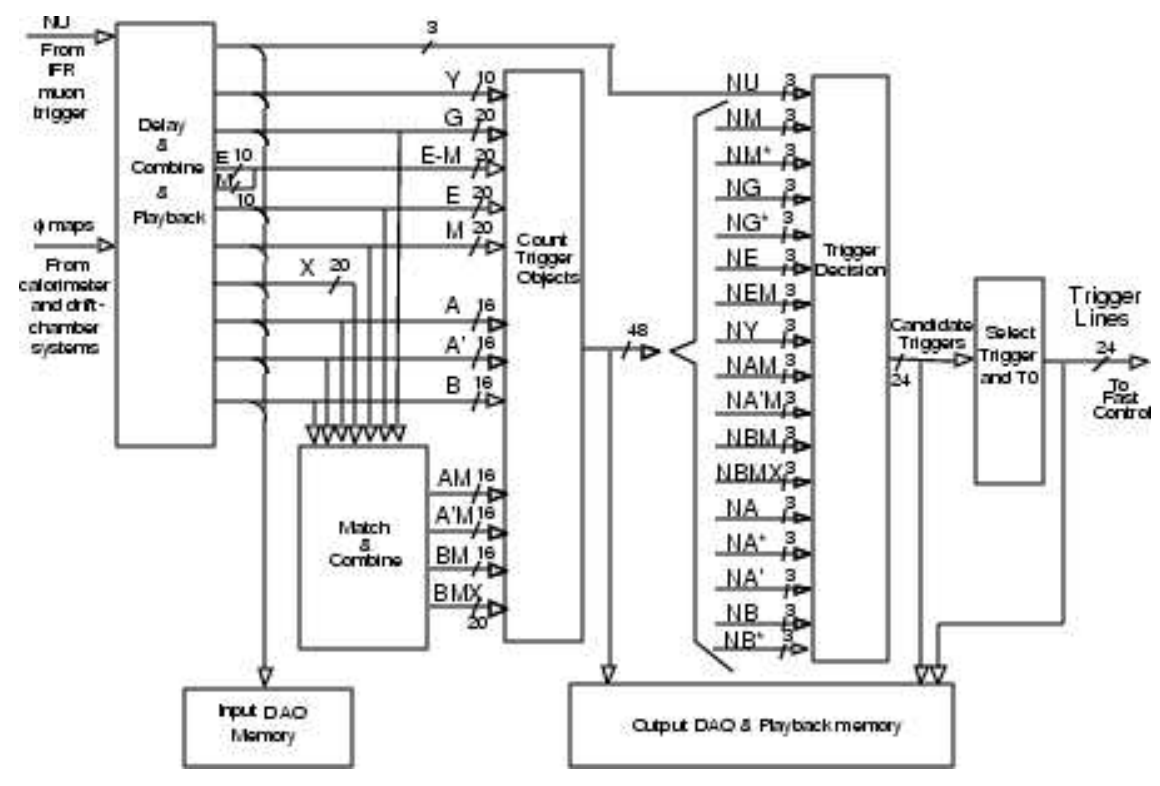

Figure 3.5: The block diagram of the global Level 1 Trigger module.

objects are tested with the preprogrammed physics conditions in logic operation, and encoded into 24 bit trigger lines.

The GLT can also derive the L1 trigger time from the center of the timing distribution of the highest priority trigger, binned in the 134 ns and spanning about $1 \mu \mathrm{s}$. Other signals compatible with this time are retained and cached. The resulting 24-bit GLT output signal is sent to the FCTS every 67ns, and the FCTS can optionally mask or prescale any of these triggers. If a valid trigger remains, a "L1 accept" signal is issued which initiates detector readouts. 


\subsection{Level 3 trigger}

Level 3 trigger takes the L1 output and the fast control scalers as input for further selection. It also has access to the raw data from the DCH and EMT. The whole process is to refine the L1 output and reject background further. The L3 software runs in an online computer farm and performs some pattern recognition with track-cluster matching. The operation of L3 is performed in three stages. The first stage is to define at least one L3 input line for each L1 output. Then with various L3 scripts, a series of different independent classification test are executed to select an event and make a decision if the events satisfies the specific selection criteria. Internally, a script may run DCH and EMC algorithms independently or together. Finally the L3 output lines are defined. The average L3 processing time is $8.5 \mathrm{~ms}$ per event per farm computer, at Luminosity of $2.6 \times 10^{33} \mathrm{~cm}^{-2} \mathrm{~s}^{-1}$.

In addition, L3 is also used for online luminosity measurement, beam energy scale monitoring, and producing calibration constants as its reconstruction algorithm is sufficiently precise to perform data-taking diagnostics.

\subsection{Level 1 DCT trigger upgrade}

During the summer of 2004, a brand new BABAR L1 DCT system, called the upgraded DCT was introduced to the online data taking system. The DCT described in section 3.2.1 is the old DCT, because only the data triggered by the old DCT is used by this analysis. For over a year, both the old and the new dct were used to insure the correct operation of the dct upgrade. The upgrade uses the drift time information 
from stereo wires in the drift chamber to perform a 3D track reconstruction that effectively rejects background events spread out along the beam line along the beam axis (z-direction).

\subsubsection{Design requirement}

The BABAR experiment, with a total integrated luminosity of $\sim 400 \mathrm{fb}^{-1}$, has been very successful. But because its goal is to gather a near $a b^{-1}$ data sample, it is critically dependent on the luminosity it can accumulate in the near future, since it is scheduled to stop collecting data in 2008 . So, it has to be able to take data at a luminosity 8 times higher than it was designed for. In order to achieve this increase in rates, the L1 DCT trigger had to be upgraded to contain the L1 trigger rate below $4 \mathrm{kHz}$, which is well within the capacity limit of the data acquisition (DAQ) system. Although other parts of DAQ was upgraded as well, the DCT upgrade was the most desirable. For this upgrade, the following requirements needed to be satisfied as well:

- It had to use the available input signals, so that no on-detector electronics modification were needed,

- L1 latency was fixed within $12.9 \mu$ s,

- it had to maintain high trigger efficiency ( $>99 \%$ for $b$ physics, and $>95 \%$ for charm physics), under acceptable background conditions.

Detailed background studies showed that a large fraction of current L1 trigger background was from interactions between the beam particles and the beam pipe at $\left|z_{0}\right| \simeq 20 \mathrm{~cm}$ from the interaction point (IP) along the $z$-axis, as illustrated in Fig 3.6. 
The existing TSF fine- $\phi$ data for both axial and stereo layers had the right resolution $(20-35 \mathrm{~mm})$ to allow an upgraded tracking trigger to discriminate track origins in $z$, and therefore this part of the background could be suppressed significantly $(>50 \%)$.

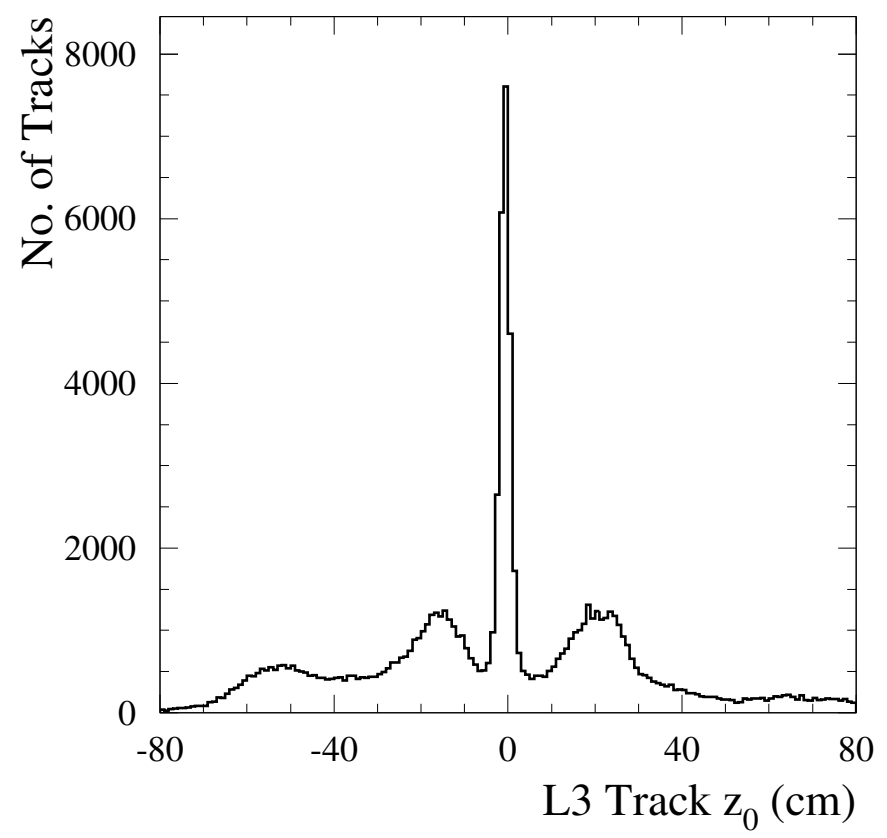

Figure 3.6: Single track z0 for tracks in all L1 triggered events, reconstructed by L3.

In hardware implementation, all the main boards except the BLT module (only use the coarse segment pattern), and interface boards needed to be redesigned. Figure 3.7 gives a schematic view on the current L1 Trigger system. 


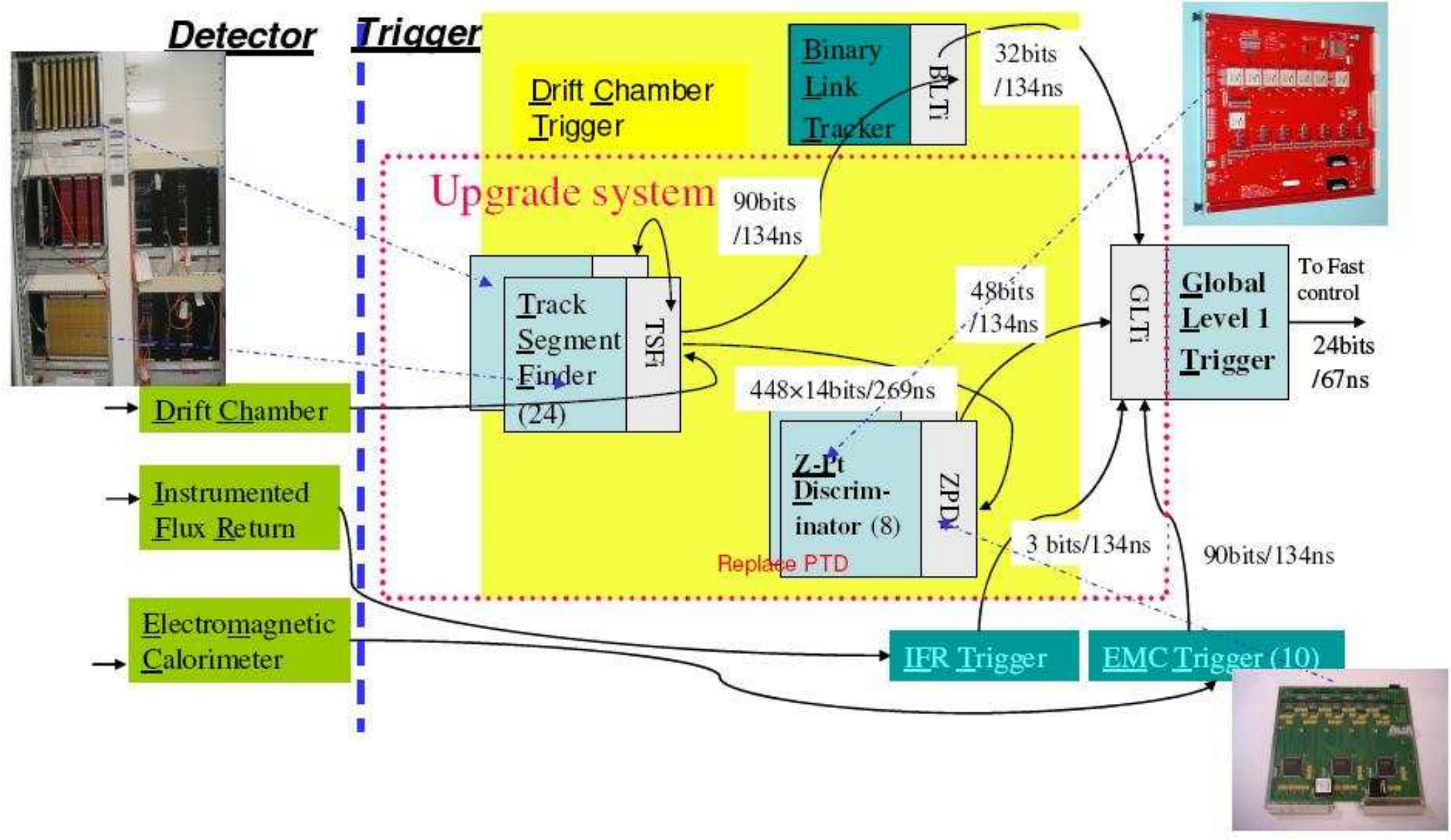

Figure 3.7: The updated trigger system. 


\subsubsection{Design implementation of the TSF modules}

As described in the section 3.2, the original TSFs have already extracted the accurate $\phi$ information for both axial and stereo segments, but only passed the axial layer $\phi$ data to the PTD modules. To make a $z$-trigger for a 3D tracking, all the 24 TSF boards needed redesigning to send both the axial and stereo information to the ZPD (defined later) up to 10 segment. The output $\phi$ resolution was increased to 6 bits from 5 bits, and output segments were augmented up to 3 per supercell PAIR.

For hardware implementation, the original 9U Euro cards with 25 XILINX FPGAs were replaced by the half $9 \mathrm{U}$ cards with five Xilinx Virtex II FPGAs. The internal logic speed was increased to $60 \mathrm{MHz}$ from $30 \mathrm{MHz}$.

\subsubsection{Design implementation of $\mathrm{z}$ and $p_{t}$ discriminator (ZPD)}

Eight ZPD modules replaced the old PTD modules which only extracted the transverse momentum $\left(p_{t}\right)$ information of the track. In the new design, each ZPD processes data from 6 TSFx and 3 TSFy modules corresponding to a $135^{\circ}$ span in $\phi$ and outputs tracks for a $45^{\circ}$ wedge (the track seed is only from two central TSFs). As a result, the ZPD modules can find the A10 track with transverse momentum $p_{t}>250 \mathrm{MeV} / c$, and the $\mathrm{A} 7$ track with transverse momentum $p_{t}>200 \mathrm{MeV} / c$ with reasonable efficiency. The total input rate is $10 \mathrm{Gbits} / \mathrm{s}$.

The ZPD track-finding algorithm is composed of two main sections, the seed track finder and the fitter. The finder selects a set of TSF segments which forms a single track out of multiple combinations by using a Hough transformation [42]. The 
fitter receives up to ten track segments associated with a track candidate. In order to ensure convergence within a fixed latency, the algorithm splits the 3-dimensional fit into 2 two-dimensional fits, thereby reducing the problem to that of linear regression. It first fits them in $r-\phi$ to improve the $1 / p_{t}$ measurement. Then it uses the difference in $\phi$ between the track and the stereo segments to fit linearly in $r-z$ to obtain $z_{0}$ and $\tan \lambda$, where $\lambda$ is the dip angle of the track w.r.t. the $r-\phi$ plane.

The Finder and Fitter algorithm engines attempt to find one seed track per seed segment in layer A7 and A10, for a total of 12 possible tracks per ZPD per 269 ns. The algorithms are implemented in six FPGAs in parallel, each processing 2 seeds every 269ns. A 75-bit multi-drop LVDS bus connects 6 finder and fitter FPGAs together at a $120 \mathrm{MHz}$ data transfer rate. The total ZPD latency is about $2 \mu s$.

The decision module implemented in a FPGA chip collects the information from the finder/fitter and make a trigger decision. More complicated trigger decisions are the combination of $p_{t}, \tan \lambda$ and $z_{0}$ cuts in conjunction with the z0 of the tracks. . Fig 3.8 shows the ZPD block diagram.

\subsubsection{Design implementation of the interface-boards}

The upgraded L1 DCT system includes three new types of interface boards: TSFi, ZPDi, and GLTi. The BLTi did not need to change, because the signals to BLT are the same as before. GLTi does not belong to the DCT system, but because the ZPD modules send more signal bits to GLT than the PTD modules, the GLTi needed to be upgrade as well. 


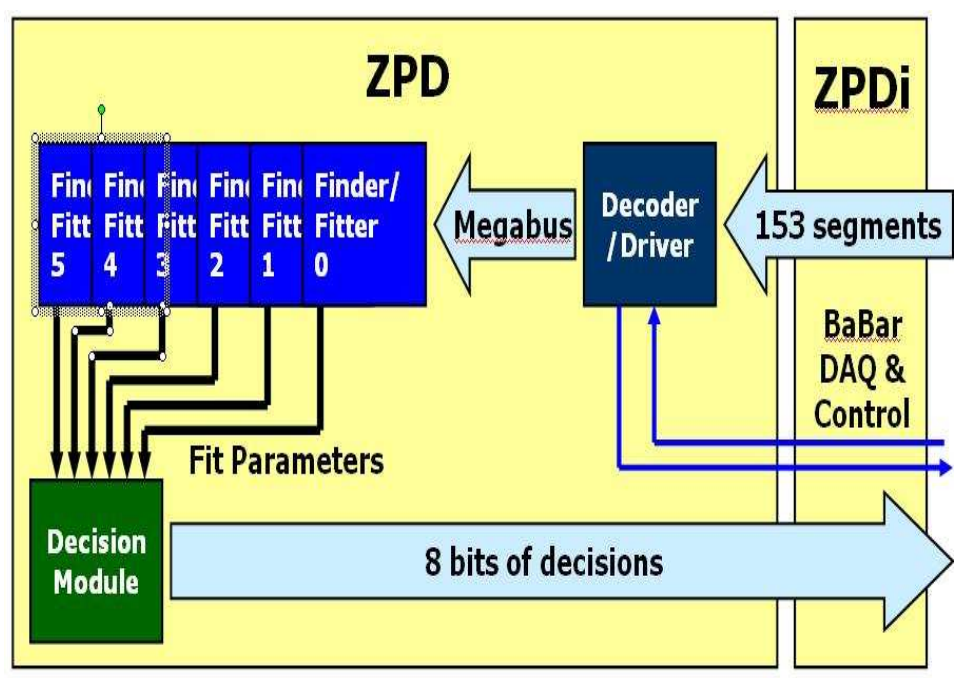

Figure 3.8: ZPD block diagram.

TSFi: A full set of 24 TSFi modules are rebuilt mainly for the new TSF to ZPD data path with a much higher data volume compared to the previous TSF to PTD data transfer volume. Each TSFi transmits same copy of data to the three ZPD modules, containing information from 21(18) segments for TSFx (TSFy). This splitter function is implemented in the FPGA hardware.

ZPDi: The ZPDi modules are the new set of interface boards for receiving data from the 9 TSFs via LVDS and providing input to the ZPDs. The TSFi modules' output signals first go through three FPGAs for masking out the unwanted segments, mapping TSF output format into ZPD input data format. Since the signals from the 9 different TSF boards are processed by three FPGAs, they have to be aligned by using the same clock signals from the ZPD modules. The FPGA chips on the ZPDi modules can also generate random pattern for ZPD tests. A ZPDi transports 6 bit of 
output from each ZPD to the GLT, while initial design only allowed a 4 bit output to the GLT. The ZPDs receive the fine- $\phi$ data via the LVDS channel link from the TSFs in 267 ns intervals, because the total data transmission rate between the TSFs and the ZPDs is about $10 G b / s$.

GLTi: The GLTi kept the number of inputs to the GLT the same as before in order to continue using the existing GLT. The ZPD contributes two 16-bits to the GLT. One signal occupies the current $A^{\prime}$ signal slot, while the other ZPD signal displaces the current EMT X signal. This required rebuilding of the GLTi, but this was achieved by simply adding connectors and being able to switch between the old and the new inputs through one FPGA chip.

An important part of the upgrade project was test of the interfaces. This involved testing all assorted parts of the overall system; not only the interface boards themselves(PCB assembly,firmware), but also the cables, backplanes, and the I/O functions of each DCT board. Most of interface tests needed to be performed with many different components involved. As an example, for the integrated ZPDi test, the functional TSF, TSFi, ZPD, GLTi and GLT needed to be included.

\subsubsection{System testing strategy}

Diagnostic memories are distributed throughout the whole system to help with troubleshooting of each subsystem. Monte Carlo events are played from input to output memory to perform bitwise validation of the implementation. These memories are also used for interface tests between boards. 
Parallel commissioning is implemented at the final stage of the system test. In 2003, the upgraded DCT was installed in BABAR and the experiment is now using the existing DCT in parallel. Fiber splitters supply the drift chamber data to both the new and current trigger systems, allowing a direct comparison between the systems without interrupting the data taking. The global L1 trigger and its interface board can switch between the new and the old L1 DCT systems.

\subsubsection{Performance of the complete DCT system}

During the Summer of 2004, 24 new TSF boards, eight production ZPD boards, and all the associated upgrade interface boards were installed into the BABAR trigger system, and used for triggering physics data in late July. The ZPD tracking
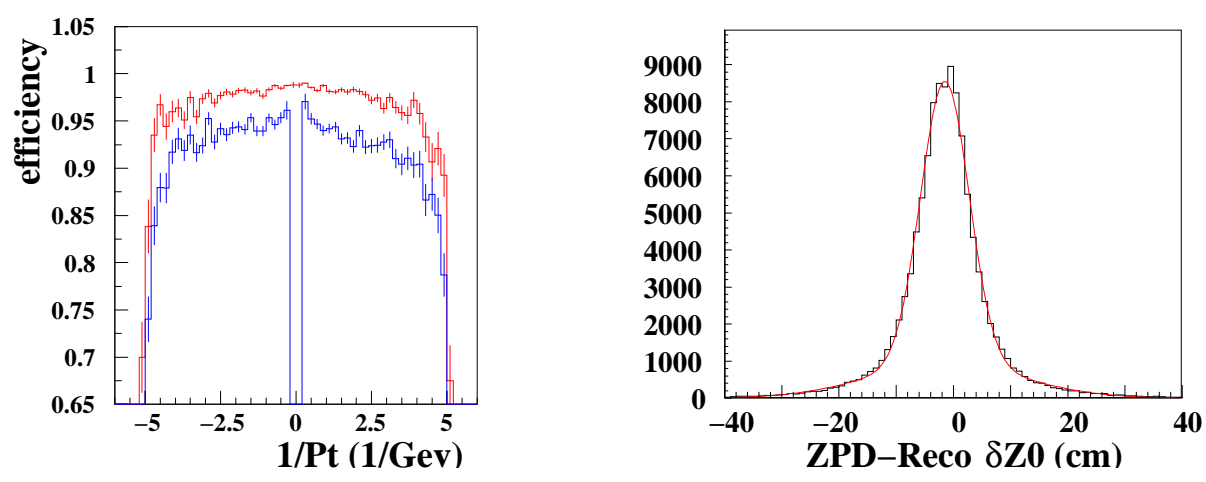

Figure 3.9: The left plot is ZPD tracking efficiency, defined as the fraction of tracks offline reconstructed as coming from the IP which pass the L1 trigger selection criteria. Upper (red) is isolated tracks, and lower (blue) is the tracks in the hadronic events. The right plot is $\mathrm{ZPD} z_{0}$ resolution, defined as the difference between ZPD reconstructed and offline reconstructed tracks. 
efficiency has been measured to be $>97 \%$ for isolated tracks and $>92 \%$ for tracks in hadronic events down to $p_{t}=250 \mathrm{MeV}$, as shown in Fig 3.9. The measured $z_{0}$ resolution is about $4.3 \mathrm{~cm}$, consistent with the expectation from Monte Carlo studies, as shown in Fig 3.9. 


\section{CHAPTER 4 TRACKING, PARTICLE ID AND VERTEXING}

\subsection{Charged track reconstruction}

In the presence of a uniform magnetic field, a moving charged particle follows a helical trajectory, a path consisting of a circular motion combined with a constant velocity motion. The track is defined by five parameters, $\left(d_{0}, \phi_{0}, \omega, z_{0}, \tan \lambda\right)$ as defined in Section 2.3.2, and their covariance error matrix. The tracking detectors, namely, the SVT and the DCH provide measurements of the track from the hits left by the charged particles. An appropriate set of the hits are selected by a pattern recognition algorithm which finds these to be consistent with belonging to a single track. This set of measurements is then fitted to a most probable helix via Kalman filter: a progressive fit algorithm [43][44] to obtain the track and the fit parameters along with the error matrix. To categorize the quality of the track-fit and reduce the background in the early stages of reconstruction, a series of track quality cuts are applied to the track to form different lists of charged tracks, such as ChargedTracks, GoodTrackVeryLoose, and GoodTrackLoose ${ }^{1}$. In this analysis, all of the charged tracks are selected from the ChargedTracks list which includes all the reconstructed tracks without extra quality cuts because some of the tracks are the decay products of long lived hyperons with displaced vertices.

\footnotetext{
${ }^{1}$ In the $B A B A R$ data structure, all the reconstructed particles are organized into lists which are groups of particles that satisfy some specific requirements
} 


\subsection{Particle identification}

Particle identification (PID) is carried out after the track and cluster reconstruction (from EMC) takes place. By using ChargedTracks list as input, stable hadrons like protons, charged kaons, and charged pions are distinguished by the combined likelihood of the specific energy-loss: $d E / d x$ in the SVT and DCH, as well as from the Cherenkov angle, number of photons emitted and the track quality in the DIRC. The likelihood for each particle hypothesis is the product of the individual likelihoods from those of the SVT, DCH and DIRC given by:

$$
\mathcal{L}_{i}=\mathcal{L}_{i}^{S V T} \mathcal{L}_{i}^{D C H} \mathcal{L}_{i}^{D I R C}
$$

where $i$ can be a proton, a kaon, or a pion. $\mathcal{L}_{i}^{S V T}$ and $\mathcal{L}_{i}^{D C H}$ are modeled by the comparison of the measured mean $d E / d x$ against the expected $d E / d x$ from the BetheBloch parameterization. The $\mathcal{L}_{i}^{D C H}$ is described by a Gaussian function, and $\mathcal{L}_{i}^{S V T}$ is assumed to be a Bifurcated Gaussian function. $\mathcal{L}_{i}^{D I R C}$ is constructed from product of two components: the number of Cheronkov photons and track quality, are described by a Poisson distribution; the difference between the measured average Cherenkov angle and the expected angle for a given mass hypothesis, is assumed to be a Gaussian distribution. Finally, based on these likelihood distributions, three likelihood ratios are calculated:

$$
\begin{aligned}
R_{1} & =\frac{\mathcal{L}_{K}}{\mathcal{L}_{K}+\mathcal{L}_{\pi}} \\
R_{2} & =\frac{\mathcal{L}_{K}}{\mathcal{L}_{K}+\mathcal{L}_{p}} \\
R_{3} & =\frac{\mathcal{L}_{p}}{\mathcal{L}_{p}+\mathcal{L}_{\pi}}
\end{aligned}
$$


Different likelihood ratio cuts are applied to form different hadron selectors: a proton candidate selector is made by applying cuts on $R_{2}$ and $R_{3}$; a kaon candidate selector is made by applying cuts on $R_{1}$ and $R_{2}$; and a pion candidate selector is made by applying cuts on $R_{1}$ and $R_{3}$. According to the different cut levels, the selectors for each particle hypothesis are categorized in four levels: VeryLoose, Loose, Tight, and VeryTight. A high momentum hadron is more likely to be misidentified as an electron as shown in Figure 2.10. Therefore, an electron rejection is added into the tighter hadron selectors. The different hadron candidates mentioned in this analysis are defined with the criteria listed in Table 4.2. In this analysis, we require the proton

Table 4.1: Criteria used for selecting available categories of hadron candidates

\begin{tabular}{lcccc}
\hline \hline category & $R_{1}$ & $R_{2}$ & $R_{3}$ & not electron \\
\hline pLHVeryLoose & & $<0.75$ & $>0.5$ & \\
KLHVeryLoose & $>0.5$ & $>0.018$ & & yes, $p>0.4 \mathrm{GeV}$ \\
KLHVeryLoose & $>0.9$ & $>0.2$ & & yes, $p>0.4 \mathrm{GeV}$ \\
\hline
\end{tabular}

candidates to satisfy the pLHVeryLoose selection; the kaon candidates are required to satisfy the KLHTight selection. Pion ID cut is not applied to any of the charged pion candidates, and only the pion mass is assigned to them. Because most of the particles are pions, this does not make any significant difference in the present study. 


\subsection{Kinematic fit and decay chain reconstruction}

As described in chapter 2 , only the stable particles $-p^{ \pm}, \pi^{ \pm}, e^{ \pm}, \mu^{ \pm}$and $\gamma$ can be directly detected by the BABAR detector. All the other particles, like charmed baryons and $B$ mesons have to be reconstructed from their decay products which leave (reconstructed) tracks or clusters. The reconstructed intermediate states are often enhanced by the application of kinematic fits. The goal of a kinematic fit is to improve the resolution of the experimental measurement and test a hypothesis by introducing various physics constraints (momentum, energy and decay vertex). Vertexing attempts to find points common to the subset of the tracks in an event, usually the decay point of the mother particle. It is very important to obtain the correct decay point as this determines the mother particle's invariant mass and momentum for the long-lived particles, thereby helping in removal of a lot of background. A Kalman filter based decay chain fitting, called VtxTreeFitter[45], is applied. It performs a least squares fit of a complete decay chain involving multiple decay vertices. It can deal with other kinematic variables, like mass constraint, beam position constraint, beam energy constraint, and lifetime constraint for obtaining a more precise result. This technique allows a simultaneous extraction of the decay time, position and momentum and their uncertainties and correlations for all particles in the decay chain.

The decay tree is made up from "bottom" to "top", i.e. from final state particles to the decay particle. To illustrate, we take the decay tree of $B^{-} \rightarrow \Xi_{c}^{0} \bar{\Lambda}_{c}^{-}$ shown in Figure 4.1 as a sample. We begin with selecting $p^{ \pm}, K^{ \pm}$and $\pi^{ \pm}$candidates from the stable particles detected by BABAR detector. Then, we combine the relevant 
candidates to give the composite immediately up in the decay tree. For example, a $p$ candidate is combined with a $\pi^{-}$candidate to make a $\Lambda^{0}$ candidate, which in turn is combined with another $\pi^{-}$candidate to form a $\Xi^{-}$candidate. At each level, we apply a set of selection criteria to reduce the background (from random combinations) while keeping the signal as high as possible. The reconstruction is performed successively up the decay tree in this manner until the $B^{-}$meson is finally reconstructed.

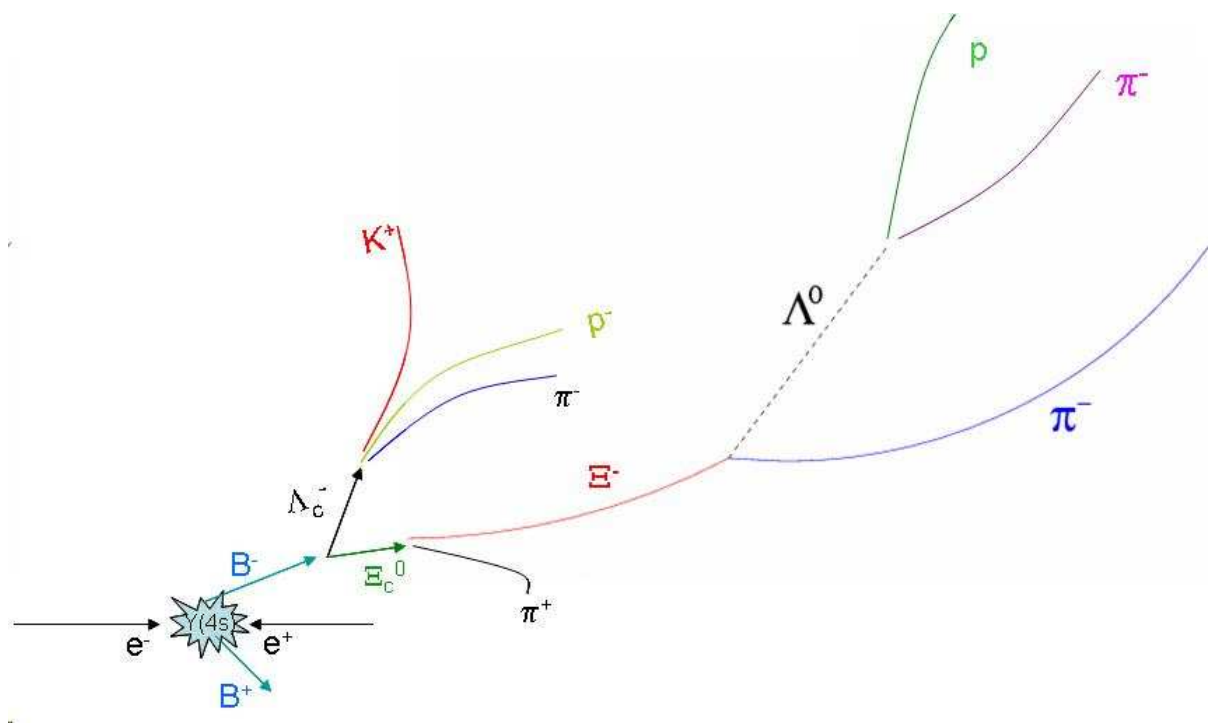

Figure 4.1: A possible decay tree for $B^{-} \rightarrow \Xi_{c}^{0} \bar{\Lambda}_{c}^{-}$ 


\section{CHAPTER 5 \\ DATA DESCIRPTION AND PRESLECTION}

\subsection{BABAR experimental data}

The data used in this analysis were collected during BABAR operation from September 2000 to July 2004 in Run 1-4, and represent about $230 \mathrm{fb}^{-1}$ of integrated luminosity. The center-of-mass $(\mathrm{CM})$ energies of the $e^{+} e^{-}$collisions were $10.58 \mathrm{GeV}$ $(\Upsilon(4 S)$ resonance) and $40 \mathrm{MeV}$ below $\Upsilon(4 S)$ resonance, referred as on-peak and offpeak data sets, respectively. Table 5.1 lists the luminosities and number of $B \bar{B}$ pairs in the subsets of the data in detail.

Table 5.1: $B A B A R$ integrated luminosity and number of $B \bar{B}$ pairs.

\begin{tabular}{l|ccc}
\hline \hline \multicolumn{1}{l|}{} & & $\mathcal{L}$ & \multicolumn{1}{c}{ Number of $B \bar{B}$} \\
\hline \multirow{3}{*}{ On resonance } & Run 1 & $17.807 \mathrm{fb}^{-1}$ & $(19.4 \pm 0.2) \times 10^{6}$ \\
& Run 2 & $59.402 \mathrm{fb}^{-1}$ & $(65.7 \pm 0.7) \times 10^{6}$ \\
& Run 3 & $30.631 \mathrm{fb}^{-1}$ & $(33.8 \pm 0.4) \times 10^{6}$ \\
& Run 4 & $99.405 \mathrm{fb}^{-1}$ & $(109.4 \pm 1.2) \times 10^{6}$ \\
& Run 1-3 & $107.84 \mathrm{fb}^{-1}$ & $(118.9 \pm 0.8) \times 10^{6}$ \\
& Run 1-4 & $207.245 \mathrm{fb}^{-1}$ & $(228.3 \pm 1.5) \times 10^{6}$ \\
\hline \multirow{3}{*}{ Off resonance } & Run 1 & $2.330 \mathrm{fb}^{-1}$ & \\
& Run 2 & $6.833 \mathrm{fb}^{-1}$ & \\
& Run 3 & $2.394 \mathrm{fb}^{-1}$ & \\
& Run 4 & $9.929 \mathrm{fb}^{-1}$ & \\
\hline \hline
\end{tabular}




\subsection{Monte Carlo simulation}

Large amounts of Monte Carlo simulated data are produced in all the interactions at the run energies with proper cross-sections and branching fractions to the best of our knowledge to date. This includes the signal decay modes where special attention is paid. The idea is to observe and measure the effect of the detector and the analysis software on the events produced at the intersection region. Early on in the experiment, the simulated collections are studied very thoroughly to see that the effects of the detector acceptance and overall efficiency match the reality and are consistent among all physics studies. The beam conditions, the detector responses and calibration are all carefully input to the simulation and updated according to the specific dataset conditions which are recorded in various databases. The analysis of thus generated data are carried out in manners identical to real data; the simulated events are processed with the same reconstruction and analysis software as the data.

There are four main steps in producing a simulated event: First, The physics of the $e^{+} e^{-}$collision is simulated by one of several event generators. The generators generate a set of four-vectors which represent the final states in the collision at the interaction point.

Second, the four-vectors from the generator stage are propagated through the exact simulation of the sub-detectors, where energy loss, production of secondaries, multiple scattering and decays can occur according to the correct probabilities. As these particles pass through sensitive regions of the detectors, energy, charge and angle information are used to calculate position and idealized energy deposits in 
the detector. These quantities, called "GHits", are stored in the database as the raw simulated data. The above task is performed within a GEANT4[46] based application.

Third, the GHits are retrieved from the database and digitized, that is, transformed into realistic signals like those collected from the detector electronics. Real background events, stored in the database, may be mixed in the simulated events to closely reproduce reality of collected data. The digitized background events are aligned in time with the simulated signal event before they are combined. The final outcome from this stage is a set of raw data objects called "digis" which are stored in the database ready for reconstruction.

Finally, these digis are retrieved from the database and combined into candidate events consisting of particle tracks, energy clusters, and particle identifications, etc., ie., That is these simulated smaples now can be treated as data.

Two types of Monte Carlo events are usually generated for a specific analysis: signal events and background events.

\subsubsection{Generic Monte Carlo}

The Generic Monte Carlo event samples contain production of $B^{0} \bar{B}^{0}, B^{+} B^{-}$, $c \bar{c}$ and $q \bar{q}(q: u, d, s)$ from $e^{+} e^{-}$in order to study these processes which are always present (mostly) as backgrounds; these are also used to optimize the event selection criteria. (These criteria are not selected by using the real data in order to minimize any bias in the the event selection procedure.) The MC samples are produced in two cycles: cycle 5 (SP5) simulating the detector condition in run 1-3, cycle 6 (SP6) 
simulating the detector condition in run 4. The total number of events generated and the corresponding effective luminosity in each category are summarized in Table 5.2 which includes SP5 and SP6. The $B^{+} B^{-}$and $B^{0} \bar{B}^{0}$ generic MC sample and the $c \bar{c}$ and $q \bar{q}(q=u, d, s)$ MC samples are about 4 times and only 1.8 times bigger that the real data sample, respectively. Even though the generic MC generates events according

Table 5.2: Generic Monte Carlo samples.

\begin{tabular}{lcccc}
\hline \hline Process & \multicolumn{2}{c}{ SP5 } & \multicolumn{2}{c}{ SP6 } \\
& $\#$ of events & $\mathcal{L}$ & \# of events & $\mathcal{L}$ \\
\hline$e^{+} e^{-} \rightarrow \Upsilon(4 S) \rightarrow B^{0} \bar{B}^{0}$ & $210,371,826$ & $382 \mathrm{fb}^{-1}$ & $336,130,399$ & $611 \mathrm{fb}^{-1}$ \\
$e^{+} e^{-} \rightarrow \Upsilon(4 S) \rightarrow B^{+} B^{-}$ & $211,576,000$ & $385 \mathrm{fb}^{-1}$ & $332,944,433$ & $605 \mathrm{fb}^{-1}$ \\
$e^{+} e^{-} \rightarrow c \bar{c}$ & $179,122,000$ & $138 \mathrm{fb}^{-1}$ & $238,798,626$ & $184 \mathrm{fb}^{-1}$ \\
$e^{+} e^{-} \rightarrow u \bar{u}+d \bar{d}+s \bar{s}$ & $311,879,000$ & $149 \mathrm{fb}^{-1}$ & $393,590,526$ & $188 \mathrm{fb}^{-1}$ \\
\hline \hline
\end{tabular}

Note: Integrated luminosities are calculated assuming cross-sections of $1.1 \mathrm{nb}, 1.3 \mathrm{nb}$, and $2.09 \mathrm{nb}$ for $B \bar{B}, c \bar{c}$, and uds, respectively.

to all the possible decay modes according to the branching fractions known from the particle data group (PDG), it does not correctly reproduce the branching fractions for different combinations of $B$ decays into charmed baryons; mainly because not much is measured in this area and the MC assumes that the $b \rightarrow c \bar{u} d$ process is the only dominant decay rather than $b \rightarrow c \bar{c} s$.

\subsubsection{Exclusive Monte Carlo production}

The signal Monte Carlo samples are used for efficiency studies, optimization of selection criteria, and for determination of the signal shapes. Their exclusive 
production, shown in Table 5.3 are generated. Because the decay mechanism is not well understood, simply phase space distribution is used in the production.

For $\bar{B} \rightarrow \Lambda_{c}^{+} \bar{\Lambda}_{c}^{-} K$ decays, each specific final state listed has its own MC dataset. But for $\bar{B} \rightarrow \Xi_{c} \bar{\Lambda}_{c}^{-}$decays, the final states listed under a specific decay mode are grouped together into sets of related channels. The last six decay modes in Table 5.3 are decay modes which can produce possible peaking backgrounds for the two-body $B$ decay modes.

In addition, some other exclusive Monte Carlo samples are generated for peaking background studies and crosschecks of efficiencies; either because they are not simulated well in the generic MC, or not simulated at all. They are listed in Table 5.4.

\subsection{Event Preselection}

Because reconstruction of the vast amount of data is resource- and timeintensive, the analysis is performed in various stages. At each stage the event sample is filtered carefully to produce a smaller data sample with desired, enriched events. Hence, the subsequent stages are lower in data volume but often more intensive in the analysis programs. The first stage is a centrally produced skim which filters all events collected from the detector and retains only events containing at least one hyperon candidate (see below). These events are then reanalyzed and only events containing at least one charmed baryon candidate are retained. This second step is called reskimming. 


\subsubsection{Skim}

Three skimmed data subsets, produced centrally, are used for the event preselection. These are referred as the LambdaVeryVeryLoose, XiMinus and LambdaC streams.

LambdaVeryVeryLoose skim: Events with at least three charged track are considered, where two oppositely charged tracks are vertexed to form a $\Lambda^{0}$ candidate. One track is identified as a proton by particle ID (pLHVeryLoose), and the other is selected from the ChargedTracks list and assigned a $\pi^{+}$mass. Events containing a $\Lambda^{0}$ candidate with a reconstructed mass between $1.106 \mathrm{GeV}$ and $1.126 \mathrm{GeV}$ are retained. The event retaining rate is about $2.4 \%$ of all events.

XiMinus skim: Once the reconstruction and selection described above for the LambdaVeryveryLoose skim are carried out, a further requirement, that the $p \pi^{-}$ invariant mass be within $10 \mathrm{MeV}$ of the nominal $\Lambda^{0}$ mass, is imposed. In these events the mass of the $\Lambda^{0}$ is then constrained to the nominal $\Lambda^{0}$ mass, refitted, and combined with a negatively charged track from the ChargedTracks list with a $\pi^{-}$mass to form $\Xi^{-}$candidates. Events containing $\Xi^{-}$candidates thus formed with a $\Lambda^{0} \pi^{-}$invariant mass within $25 \mathrm{MeV}$ of the nominal PDG value are selected to form the XiMinus skim sample. The event retainment rate in data is about $0.5 \%$ of all events.

LambdaC skim: The $\Lambda_{c}^{+}$candidates are reconstructed using the Cascade vertexer in the following seven decay final states: $p^{+} K^{-} \pi^{+}, p^{+} K_{S}^{0}, p^{+} K_{S}^{0} \pi^{+} \pi^{-}, \Lambda^{0} \pi^{+}$, $\Lambda^{0} \pi^{+} \pi^{+} \pi^{-}, \Sigma^{0} \pi^{+}$and $\Sigma^{0} \pi^{+} \pi^{+} \pi^{-}$. Events with at least one $\Lambda_{c}^{+}$candidate in the seven final states are selected. The event retention rate is about $1.9 \%$ of all events. 


\subsubsection{Reskimming}

Reskimming is the second stage of event preselection, designed to select events with one or two charmed baryons. Minimal selection cuts are still used at this stage to retain maximum efficiencies. A total of four different reskimmed data subsets are created from both the data and the generic Monte Carlo samples; these are the $\Xi_{c}^{0} \rightarrow \Xi^{-} \pi^{+}, \Xi_{c}^{0} \rightarrow \Lambda^{0} K^{-} \pi^{+}, \Xi_{c}^{+} \rightarrow \Xi^{-} \pi^{+} \pi^{+}$, and the $\Lambda_{c}^{+} \bar{\Lambda}_{c}^{-}$skims. The generic Monte Carlo samples, as mentioned earlier, contain $B^{0} \bar{B}^{0}, B^{+} B^{-}$and the continuum events: $u \bar{u}, d \bar{d}, s \bar{s}$, and $c \bar{c}$.

$$
\text { 5.3.2.1 } \Xi_{c}^{0} \rightarrow \Xi^{-} \pi^{+}
$$

The $\Xi_{c}^{0} \rightarrow \Xi^{-} \pi^{+}$selection starts from the XiMinus skim. The $\Xi^{-}$are reconstructed as described above, and the $\pi^{+}$are taken from the ChargedTracks list. The following additional selection criteria are applied:

- The invariant mass for $\Lambda^{0} \rightarrow p^{+} \pi^{-}$must lie between 1.106 and $1.125 \mathrm{GeV} / c^{2}$ (the $\Lambda^{0}$ mass is then constrained to the nominal $\Lambda^{0}$ mass as before).

- The invariant mass of $\Xi^{-} \rightarrow \Lambda^{0} \pi^{-}$between 1.308 and $1.336 \mathrm{GeV} / c^{2}$, and no mass constraint.

- The invariant mass of $\Xi_{c}^{0} \rightarrow \Xi^{-} \pi^{+}$must lie between 2.370 and $2.572 \mathrm{GeV} / c^{2}$ (a large mass range is selected to allow for a background study).

- The vertex probability for the whole decay tree must satisfy $P\left(\chi^{2}\right)>0.01 \%$. 
The event retaining rate for the reskimming is between $4 \%$ and $6 \%$ with a relative skim efficiency of nearly $100 \%$.

$$
\text { 5.3.2.2 } \Xi_{c}^{0} \rightarrow \Lambda^{0} K^{-} \pi^{+}
$$

The $\Xi_{c}^{0} \rightarrow \Lambda^{0} K^{-} \pi^{+}$selection starts with the LambdaVeryVeryLoose skim. The $\Lambda^{0}$ is reconstructed as described above, and the $\pi^{+}$is taken from the ChargedTracks list. We apply the following additional selection criteria:

- The kaon must pass KLHVeryLoose particle identification criteria.

- The invariant mass for $\Lambda^{0} \rightarrow p^{+} \pi^{-}$must lie between 1.106 and $1.125 \mathrm{GeV} / c^{2}$ (the mass is then constrained to the nominal mass as before).

- The invariant mass for the $\Xi_{c}^{0} \rightarrow \Lambda^{0} K^{-} \pi^{+}$must lie between 2.30 and $2.64 \mathrm{GeV} / c^{2}$ (a large mass range is selected to allow for background study).

- The vertex probability for the whole decay tree must satisfy $P\left(\chi^{2}\right)>0.01 \%$

The retaining rate for the reskimming is $\sim 4 \%$ with a relative skim efficiency of close to $100 \%$.

$$
\text { 5.3.2.3 } \Xi_{c}^{+} \rightarrow \Xi^{-} \pi^{+} \pi^{+}
$$

The $\Xi_{c}^{+} \rightarrow \Xi^{-} \pi^{+} \pi^{+}$selection also starts with the $\Xi^{-}$skim. All the selection criteria are the same as in the $\Xi_{c}^{0} \rightarrow \Xi^{-} \pi^{+}$skim except that one more pion is added. The event retaining rate of the reskimming, is between $9 \%$ and $12 \%$, with a relative skim efficiency of about $100 \%$. 


\subsubsection{4 $\Lambda_{c}^{+} \bar{\Lambda}_{c}^{-}$}

The analysis of $B^{-} \rightarrow \Lambda_{c}^{+} \bar{\Lambda}_{c}^{-} K^{-}$and $\bar{B}^{0} \rightarrow \Lambda_{c}^{+} \bar{\Lambda}_{c}^{-} K_{s}^{0}$ decays requires the presence of at least two $\Lambda_{c} \mathrm{~s}$ in each event. The candidate selection for the skim is based on the LambdaC skim; one $\Lambda_{c}^{+}$and one $\bar{\Lambda}_{c}^{-}$are combined. No $\Lambda_{c}^{+}$reconstruction is needed, since the $\Lambda_{c}^{+}$candidates are present (persistent) in the BetaCandidate list in the LambdaC skim and are directly used for the current analysis. The event retaining rate for this skimming is between $2 \%$ and $2.5 \%$ with a relative skim efficiency of about $100 \%$. 
Table 5.3: Signal Monte Carlo samples.

\begin{tabular}{|c|c|c|}
\hline Decay Chain & Number of events & SP mode number \\
\hline \multicolumn{3}{|l|}{$B^{-} \rightarrow \Lambda_{c}^{+} \bar{\Lambda}_{c}^{-} K^{-}$} \\
\hline$\Lambda_{c}^{+} \rightarrow p^{+} K^{-} \pi^{+}, \bar{\Lambda}_{c}^{-} \rightarrow p^{-} K^{+} \pi^{-}$ & 117,000 & 6620 \\
\hline$\Lambda_{c}^{+} \rightarrow p^{+} K^{-} \pi^{+}, \bar{\Lambda}_{c}^{-} \rightarrow p^{-} K_{S}^{0}$ & 117,000 & 6621 \\
\hline$\Lambda_{c}^{+} \rightarrow p^{+} K^{-} \pi^{+}, \bar{\Lambda}_{c}^{-} \rightarrow \bar{\Lambda}^{0} \pi^{-}$ & 117,000 & 6622 \\
\hline$\Lambda_{c}^{+} \rightarrow p^{+} K_{S}^{0}, \bar{\Lambda}_{c}^{-} \rightarrow p^{-} K^{+} \pi^{-}$ & 117,000 & 6623 \\
\hline$\Lambda_{c}^{+} \rightarrow \Lambda^{0} \pi^{+}, \bar{\Lambda}_{c}^{-} \rightarrow p^{-} K^{+} \pi^{-}$ & 117,000 & 6624 \\
\hline \multicolumn{3}{|l|}{$\bar{B}^{0} \rightarrow \Lambda_{c}^{+} \bar{\Lambda}_{c}^{-} K_{s}^{0}$} \\
\hline$\Lambda_{c}^{+} \rightarrow p^{+} K^{-} \pi^{+}, \bar{\Lambda}_{c}^{-} \rightarrow p^{-} K^{+} \pi^{-}$ & 117,000 & 6625 \\
\hline $\begin{array}{l}\bar{B}^{0} \rightarrow \Xi_{c}^{+} \bar{\Lambda}_{c}^{-} \\
\Xi_{c}^{+} \rightarrow \Xi^{-} \pi^{+} \pi^{+}, \bar{\Lambda}_{c}^{-} \rightarrow p^{-} K^{+} \pi^{-} \\
\Xi_{c}^{+} \rightarrow \Xi^{-} \pi^{+} \pi^{+}, \bar{\Lambda}_{c}^{-} \rightarrow p^{-} K_{S}^{0} \\
\Xi_{c}^{+} \rightarrow \Xi^{-} \pi^{+} \pi^{+}, \bar{\Lambda}_{c}^{-} \rightarrow \bar{\Lambda}^{0} \pi^{-}\end{array}$ & 231,000 & 6021 \\
\hline $\begin{array}{l}B^{-} \rightarrow \Xi_{c}^{0} \bar{\Lambda}_{c}^{-} \\
\Xi_{c}^{0} \rightarrow \Xi^{-} \pi^{+}, \bar{\Lambda}_{c}^{-} \rightarrow p^{-} K^{+} \pi^{-} \\
\Xi_{c}^{0} \rightarrow \Xi^{-} \pi^{+}, \bar{\Lambda}_{c}^{-} \rightarrow p^{-} K_{S}^{0} \\
\Xi_{c}^{0} \rightarrow \Xi^{-} \pi^{+}, \bar{\Lambda}_{c}^{-} \rightarrow \bar{\Lambda}^{0} \pi^{-} \\
\Xi_{c}^{0} \rightarrow \Lambda^{0} K^{-} \pi^{+}, \bar{\Lambda}_{c}^{-} \rightarrow p^{-} K^{+} \pi^{-} \\
\Xi_{c}^{0} \rightarrow \Lambda^{0} K^{-} \pi^{+}, \bar{\Lambda}_{c}^{-} \rightarrow p^{-} K_{S}^{0} \\
\Xi_{c}^{0} \rightarrow \Lambda^{0} K^{-} \pi^{+}, \bar{\Lambda}_{c}^{-} \rightarrow \bar{\Lambda}^{0} \pi^{-}\end{array}$ & 288,000 & 6027 \\
\hline $\begin{array}{l}\bar{B}^{0} \rightarrow \Xi_{c}^{\prime}+\bar{\Lambda}_{c}^{-} \\
\Xi_{c}^{\prime+} \rightarrow \Xi_{c}^{+} \gamma, \bar{\Lambda}_{c}^{-} \rightarrow p^{-} K^{+} \pi^{-} \\
\Xi_{c}^{\prime+} \rightarrow \Xi_{c}^{+} \gamma, \bar{\Lambda}_{c}^{-} \rightarrow p^{-} K_{S}^{0} \\
\Xi_{c}^{\prime+} \rightarrow \Xi_{c}^{+} \gamma, \bar{\Lambda}_{c}^{-} \rightarrow \bar{\Lambda}^{0} \pi^{-}\end{array}$ & 117,000 & 6020 \\
\hline $\begin{array}{l}B^{-} \rightarrow \Xi_{c}^{\prime} 0 \bar{\Lambda}_{c}^{-} \\
\Xi_{c}^{\prime 0} \rightarrow \Xi_{c}^{0} \gamma, \bar{\Lambda}_{c}^{-} \rightarrow p^{-} K^{+} \pi^{-} \\
\Xi_{c}^{\prime 0} \rightarrow \Xi_{c}^{0} \gamma, \bar{\Lambda}_{c}^{-} \rightarrow p^{-} K_{S}^{0} \\
\Xi_{c}^{\prime 0} \rightarrow \Xi_{c}^{0} \gamma, \bar{\Lambda}_{c}^{-} \rightarrow \bar{\Lambda}^{0} \pi^{-}\end{array}$ & 117,000 & 6026 \\
\hline $\begin{array}{l}\bar{B}^{0} \rightarrow \Xi_{c}^{0} \bar{\Lambda}_{c}^{-} \pi^{+} \\
\text {includes intermediate resonances }\end{array}$ & 117,000 & 6023 \\
\hline $\begin{array}{l}B^{-} \rightarrow \Xi_{c}^{+} \bar{\Lambda}_{c}^{-} \pi^{-} \\
\text {includes intermediate resonances }\end{array}$ & 117,000 & 6024 \\
\hline $\begin{array}{l}\bar{B}^{0} \rightarrow \Xi_{c} \bar{\Lambda}_{c}^{-} n \pi^{+}, n=2,3 \\
\text { includes intermediate resonances }\end{array}$ & 117,000 & 6022 \\
\hline $\begin{array}{l}B^{-} \rightarrow \Xi_{c} \bar{\Lambda}_{c}^{-} n \pi^{-}, n=2,3 \\
\text { includes intermediate resonances }\end{array}$ & 117,000 & 6025 \\
\hline
\end{tabular}


Table 5.4: Summary of private MC

\begin{tabular}{lc}
\hline \hline Decay Chain & Number of events \\
\hline$B^{-} \rightarrow \Lambda_{c}^{+} \bar{\Lambda}_{c}^{-} K^{-}, \Lambda_{c}^{+} \rightarrow \Delta^{++} K^{-}, \bar{\Lambda}_{c}^{-} \rightarrow p^{-} K^{* 0}$ & $20 \mathrm{k}$ \\
\hline$B^{-} \rightarrow \Lambda_{c}^{+} \bar{\Lambda}_{c}^{-} K^{-}, \Lambda_{c}^{+} \rightarrow p^{+} K^{-} \pi^{+}, \bar{\Lambda}_{c}^{-} \rightarrow p^{-} K^{* 0}$ & $20 \mathrm{k}$ \\
\hline$B^{-} \rightarrow \Lambda_{c}^{+} \bar{\Lambda}_{c}^{-} K^{-}, \Lambda_{c}^{+} \rightarrow p^{+} K^{-} \pi^{+}, \bar{\Lambda}_{c}^{-} \rightarrow \Delta^{--} K^{+}$ & $20 \mathrm{k}$ \\
\hline$B^{-} \rightarrow \Lambda_{c}^{+} \bar{\Lambda}_{c}^{-} K^{-}, \Lambda_{c}^{+} \rightarrow p^{+} K^{-} \pi^{+}, \bar{\Lambda}_{c}^{-} \rightarrow p^{-} K^{+} \pi^{-}$ & $20 \mathrm{k}$ \\
\hline$B^{-} \rightarrow \Xi_{c}^{0} \bar{\Lambda}_{c}^{-}, \Xi_{c}^{0} \rightarrow \Xi^{-} \pi^{+}, \Lambda_{c}^{+} \rightarrow p^{+} K^{-} \pi^{+}$ & $20 \mathrm{~K}$ \\
\hline$B^{-} \rightarrow \Xi_{c}^{0} \bar{\Lambda}_{c}^{-}, \Xi_{c}^{0} \rightarrow \Xi^{-} \pi^{+}, \Lambda_{c}^{+} \rightarrow p^{+} K^{* 0}$ & $20 \mathrm{~K}$ \\
\hline$B^{-} \rightarrow \Xi_{c}^{0} \bar{\Lambda}_{c}^{-}, \Xi_{c}^{0} \rightarrow \Xi^{-} \pi^{+}, \Lambda_{c}^{+} \rightarrow \Delta^{++} K^{-}$ & $20 \mathrm{~K}$ \\
\hline $\bar{B}^{-} \rightarrow \Xi_{c}^{0} \Delta^{--} K^{+}$ & $10 \mathrm{~K}$ \\
\hline$B^{-} \rightarrow \Xi_{c}^{0} p^{-} K^{+} \pi^{-}$ & $10 \mathrm{~K}$ \\
\hline$B^{-} \rightarrow \Lambda_{c}^{+} p^{-} K^{+} \pi^{-} K^{-}$ & $10 \mathrm{k}$ \\
\hline$B^{-} \rightarrow p^{+} K^{-} \pi^{+} p^{-} K^{+} \pi^{-} K^{-}$ & $10 \mathrm{k}$ \\
\hline $\bar{B}^{0} \rightarrow \Xi_{c}^{+} \bar{\Lambda}_{c}^{-} \pi^{+} \pi^{-} \pi^{0}$ & $30 \mathrm{k}$ \\
\hline $\bar{B}^{0} \rightarrow \Xi_{c}^{+} \Sigma_{c}^{-}$ & $20 \mathrm{k}$ \\
\hline $\bar{B}^{0} \rightarrow \Xi_{c}^{+} \Sigma_{c}^{-} \pi^{0}$ & $20 \mathrm{k}$ \\
\hline$B^{-} \rightarrow \Xi_{c}^{0} \bar{\Lambda}_{c}^{-} \pi^{0}$ & $12 \mathrm{k}$ \\
\hline$B^{-} \rightarrow \Xi_{c}^{0} \Sigma_{c}^{-}$ & $12 \mathrm{k}$ \\
\hline$B^{-} \rightarrow \Xi_{c}^{* 0} \Sigma_{c}^{-}$ & $30 \mathrm{k}$ \\
\hline$B^{-} \rightarrow \Xi_{c}^{* 0} \Sigma_{c}^{-} \pi^{0}$ & $20 \mathrm{k}$ \\
\hline \hline $\bar{c} \rightarrow \Xi_{c}^{0} \bar{\Lambda}_{c}^{-}$ & $10 \mathrm{k}$ \\
\hline$c \bar{c} \rightarrow \Lambda_{c}^{+} \bar{\Lambda}_{c}^{-}$ & $10 \mathrm{k}$ \\
\hline$B^{0} \rightarrow \Xi_{c}^{0}+X, \bar{B}^{0} \rightarrow \bar{\Lambda}_{c}^{-} p^{+} \pi^{+} \pi^{-}$ & $10 \mathrm{k}$ \\
\hline$B^{0} \rightarrow \Xi_{c}^{0}+X, \bar{B}^{0} \rightarrow \bar{\Lambda}_{c}^{-} p^{+}$ & $10 \mathrm{k}$ \\
\hline \hline
\end{tabular}




\section{CHAPTER 6 \\ EVENT SELECTION}

\subsection{Event Reconstruction and Selection}

Using the events that pass the preselection described in section 3.2, we now reconstruct $B$ meson candidates in the final states shown in Table 1.6; details of the reconstruction and selection procedures are described below. Candidates passing all cuts are written out as ROOT ntuples. The TreeFitter vertexer is used throughout.

\subsubsection{Long-lived $K_{S}^{0}, \Lambda$ and $\Xi^{-}$reconstruction and selection}

The intermediate particles $K_{S}^{0}$, and $\Lambda$ and $\Xi^{-}$are all long-lived with $(c \tau)_{K_{S}^{0}}=$ $2.68 \mathrm{~cm},(c \tau)_{\Lambda}=7.89 \mathrm{~cm}$ and $(c \tau)_{\Xi^{-}}=4.91 \mathrm{~cm}$. So, their decay vertices are displaced from the primary vertex of the event.

\subsubsection{1 $\Lambda^{0}$ reconstruction}

The $\Lambda^{0}$ candidates are reconstructed in $p^{+} \pi^{-}$final state which has a branching fraction of $\mathcal{B}\left(\Lambda^{0} \rightarrow p^{+} \pi^{-}\right)=63.9 \pm 0.5 \%[47]$. The $\Lambda^{0}$ candidates are formed as described in section 4.1. The invariant mass distribution of an inclusive sample of the $\Lambda^{0}$ candidates from a data sample of $15 \mathrm{fb}^{-1}$ (real data) is shown in the left in the Figure 6.1. Also shown is a fit to the distribution with the combination of a double Gaussian (for the signal) and a first order polynomial (for the background). The $\sigma$ of the core Gaussian is $0.768 \pm 0.003 \mathrm{MeV}$ with a fraction of $53.8 \%$ of the area, and the $\sigma$ of wide Gaussian is about $2.13 \pm 0.01 \mathrm{MeV}$. The effective resolution 

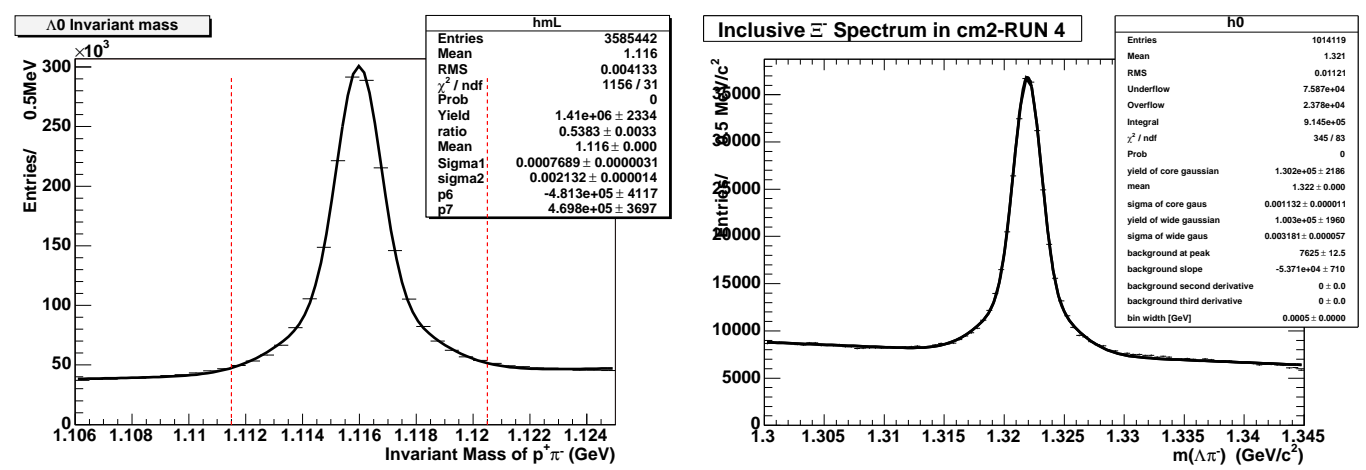

Figure 6.1: Invariant mass spectra of the intermediate hyperon from representative subsamples in data. The left plot shows the invariant mass distribution of $\Lambda^{0}\left(p K \pi^{+}\right)$ from $15 \mathrm{fb}^{-1}$ data; and the plot on the right side shows the invariant mass distribution of $\Xi^{-}\left(\Lambda^{0} \pi^{-}\right)$from $99 \mathrm{fb}^{-1}$ data.

$\sigma_{E f f}=\sqrt{f_{1} \sigma_{1}^{2}+f_{2} \sigma_{2}^{2}}$ is $1.5 \mathrm{MeV}$. The mean of the Gaussian is $1115.983 \pm 0.001 \mathrm{MeV}$. The sample of the $\Lambda^{0}$ candidates is used for the modes $\Xi^{-} \rightarrow \Lambda^{0} \pi^{-}, \Xi_{c}^{0} \rightarrow \Lambda^{0} K^{-} \pi^{+}$ and $\Lambda_{c}^{+} \rightarrow \Lambda^{0} \pi^{+}$as described below. For these modes, the mass of $\Lambda^{0}$ candidate is required to lie within $3 \sigma( \pm 4.5 \mathrm{MeV})$ of its fitted mean mass value. The $\Lambda^{0}$ mass is then constrained to its nominal value.

\subsubsection{2 $\Xi^{-}$reconstruction}

The $\Xi^{-}$candidates are reconstructed in the final state $\Lambda^{0} \pi^{+}$which has a branching fraction $\mathcal{B}\left(\Xi^{-} \rightarrow \Lambda^{0} \pi^{-}\right)=(99.8887 \pm 0.035) \%$ [47]. The transverse decay length of the $\Xi^{-}$candidate is required to be more than $0.2 \mathrm{~cm}$ to remove backgrounds from primary vertex (Xim direct production as opposed to from charmed baryon decays) to improve signal-to-background ratio. The $\Xi^{-}$candidate is also required to travel outwards from the primary vertex: the transverse distance from the primary 
vertex to the decay point of the $\Lambda^{0}$ is required to be greater than that of the $\Xi^{-}$ candidate. The inclusive invariant mass distribution of the $\Xi^{-}$candidates is shown at right in Fig 6.1 from a sample of $99 \mathrm{fb}^{-1}$ of on-peak data. A fit to the distribution with a double Gaussian (for signal) and a first order polynomial (for background) is superimposed. The $\sigma$ of the core Gaussian is $1.13 \pm 0.01 \mathrm{MeV}$ with a fraction of $56.5 \%$ of the area, and the $\sigma$ of the wide Gaussian is $3.18 \pm 0.05 \mathrm{MeV}$. The effective resolution is $\sigma_{\text {eff }}=2 \mathrm{MeV}$. The mean of the Gaussian is $1321.95 \pm 0.04 \mathrm{MeV}$. The $\Xi^{-}$is then used for the reconstruction of $\Xi_{c}^{+} \rightarrow \Xi^{-} \pi^{+} \pi^{+}, \Xi_{c}^{0} \rightarrow \Xi^{-} \pi^{+}$as described below. For these modes, the mass of the $\Xi^{-}$candidate is required to lie within $3 \sigma$ of its fitted mean mass value. The $\Xi^{-}$mass is then constrained to its nominal value.

Fig $6.2(\mathrm{a})$ shows the rate of raw yield of the reconstructed $\Xi^{-}$over the data taking period for a sample of all the data from Run4. The rate is observed to decrease with time, resulting in a possible decrease in the $\Xi_{c}^{0}$ rate in turn. This has been studied in detail. We also study the variation in DCH and SVT hits with time to investigate the cause and observe a decrease in the number of DCH hits in Run4.

\subsubsection{3 $K_{S}^{0}$ selection}

The $K_{S}^{0}$ candidates are reconstructed and selected by computing the invariant mass of two oppositely charged tracks, $\pi^{+} \pi^{-}$, forming a vertex and requiring the mass to be between $0.485 \mathrm{GeV}$ and $0.510 \mathrm{GeV}$. The $\chi^{2}$ of the vertex fit has to have a probability higher than $0.01 \%$. The cosine of the angle between the displacement vector $(\vec{r})$ and the momentum vector $(\vec{v})$ for the $K_{S}^{0}$ candidate is required to be greater 


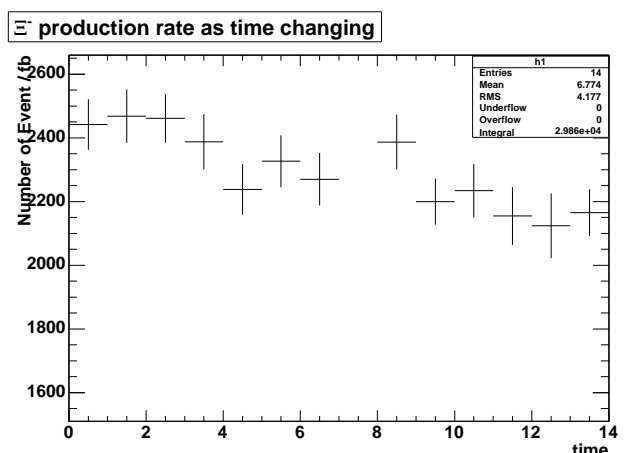

(a)

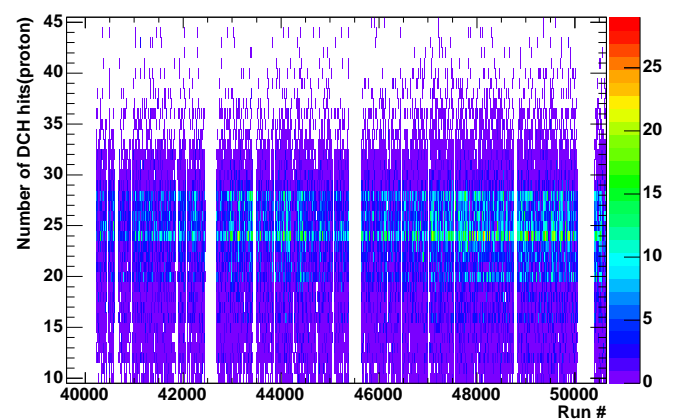

(b)

Figure 6.2: The variation of $\Xi^{-}$raw yield (left) and number of DCH hits (right) over the time periods.

than 0 , and the component of $\vec{r}$ transverse to the beam axis is required to be greater than $0.2 \mathrm{~cm}$. The momentum spectra of the $K_{S}^{0}$ candidates from $\Lambda_{c}^{+} \rightarrow p^{+} K_{S}^{0}$ and from $\bar{B}^{0} \rightarrow \Lambda_{c}^{+} \bar{\Lambda}_{c}^{-} K_{s}^{0}$, are quite different as shown in Figure 6.3 ; the $K_{S}^{0}$ 's from the $B$ decays are softer, as expected.

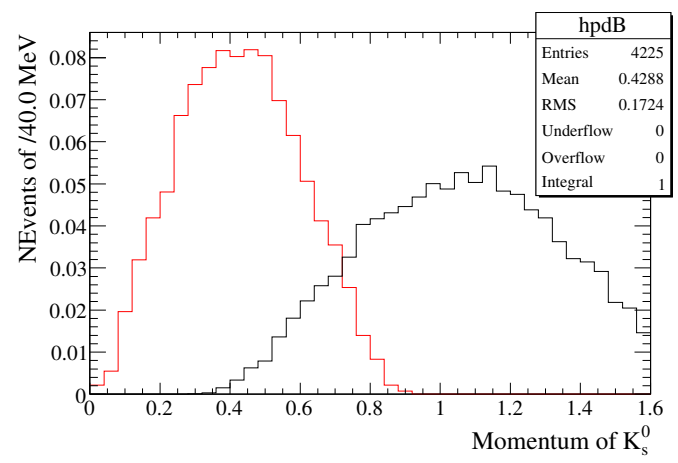

Figure 6.3: Momentum distribution of $K_{S}^{0}$ in the signal Monte Carlo, red line represents the momentum distribution of the $K_{S}^{0}$ decayed from $B$, black line represents the momentum distribution of the $K_{S}^{0}$ decayed from $\Lambda_{c}^{+}$. 


\subsection{2 $\Xi_{c}^{0}$ reconstruction and selection}

The $\Xi_{c}^{0}$ is reconstructed in two decay final states: $\Xi^{-} \pi^{+}$and $\Lambda^{0} K^{-} \pi^{+}$. Although the absolute branching fractions are not known, the ratio of the branching fractions has previously been measured as $\frac{\Gamma\left(\Xi_{c}^{0} \rightarrow \Lambda^{0} K^{-} \pi^{+}\right)}{\Gamma\left(\Xi_{c}^{0} \rightarrow \Xi^{-} \pi^{+}\right)}=1.07 \pm 0.12 \pm 0.07[48]$.

\subsubsection{1 $\Xi_{c}^{0} \rightarrow \Xi^{-} \pi^{+}$reconstruction and selection}

We combine a $\Xi^{-}$candidate which satisfied the selection criteria described in previous section, and a $\pi^{+}$candidate, to from a $\Xi_{c}^{0}$ candidate. The $\chi^{2}$ of the vertex fit should satisfy a probability greater than $0.01 \%$. Figure 6.4 shows the invariant mass distribution of the $\Xi^{-} \pi^{+}$from $59 \mathrm{fb}^{-1}$ on-peak data (left) and from the signal $\mathrm{MC}$ (right). The mass distribution in the data is fitted to a single Gaussian with a $\sigma$ of $6.07 \pm 0.18 \mathrm{MeV}$.
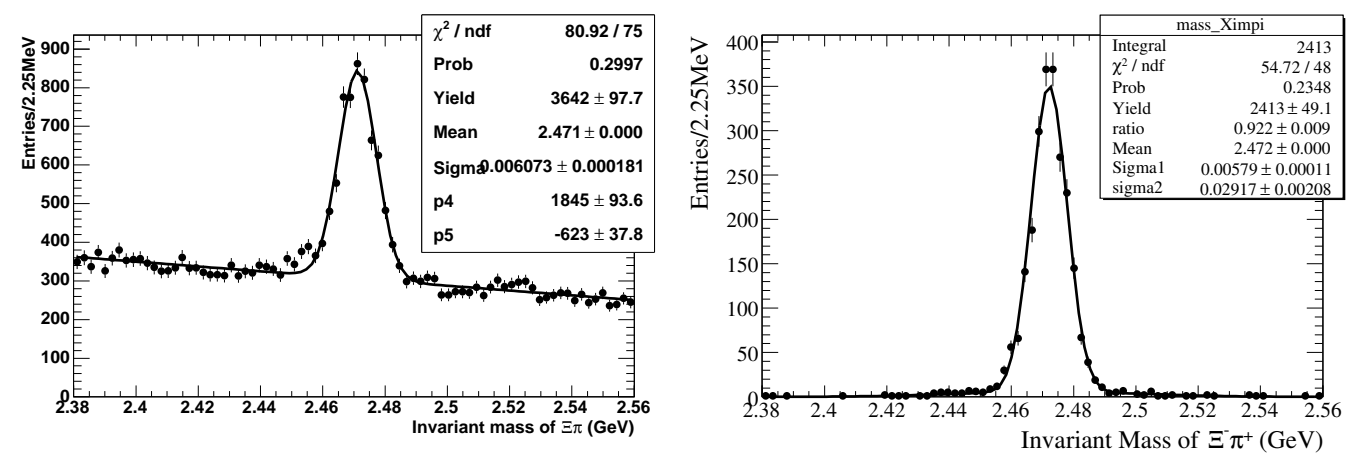

Figure 6.4: Invariant Mass distribution of $\Xi_{c}^{0} \rightarrow \Xi^{-} \pi^{+}$candidates from the BABAR data (left), and from truth-matched signal MC (right). On the left, the signal is fitted with a single Gaussian, where the fitted mean is $2471.15 \pm 0.18 \mathrm{MeV}$ with $\sigma=6.07 \pm 0.18 \mathrm{MeV}$. On the right, the signal is fitted with a double-Gaussian; the fitted parameters are mean $=2472.16 \pm 0.13 \mathrm{MeV}$, core Gaussian $\sigma_{1}=5.79 \pm 0.11 \mathrm{MeV}$ with $92.2 \pm 0.9 \%$ of the yield, and $\sigma_{2}=29.17 \pm 2.08 \mathrm{MeV}$. 
We also measure the $p^{*}$ (the 3 -momentum in the $e^{+} e^{-}$rest frame) distribution of the $\Xi_{c}^{0}$ 's from the on-peak data, shown in Figure 6.5 , where the $\Xi_{c}^{0}$ signal region is selected within a mass window $\pm 3 \sigma$ of its central mass value. A clear two-peak structure is visible in Figure $6.5(\mathrm{~b})$, which shows the $p^{*}$ distribution after background subtraction. The lower peak below $p^{*}<1.5 \mathrm{GeV}$ is primarily from the $\Xi_{c}^{0}$ produced in $B$ meson decays, and the upper peak represents the $\Xi_{c}^{0}$ produced from the $c \bar{c}$ continuum.
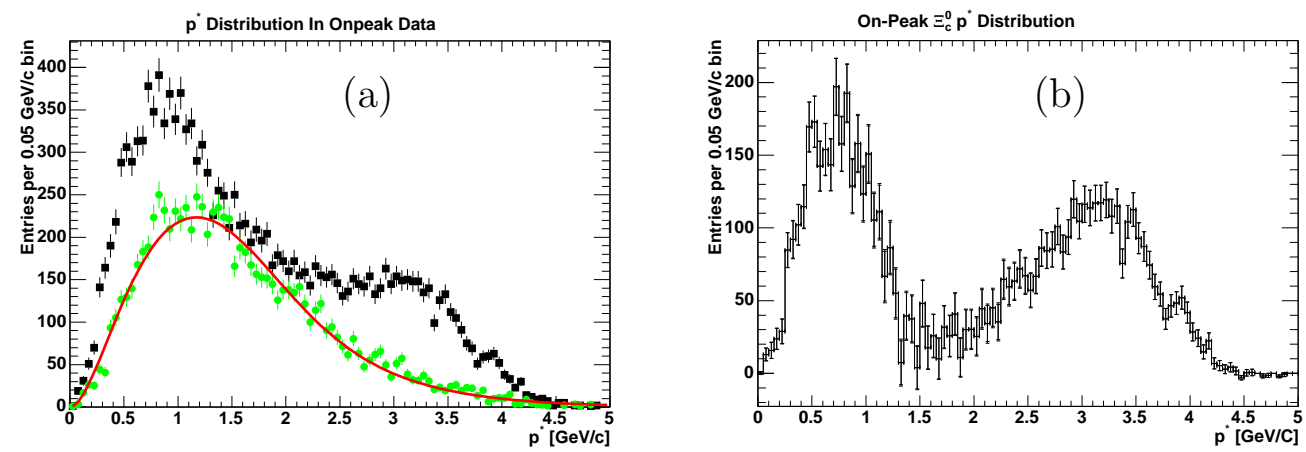

Figure 6.5: $p^{*}$ distribution from reconstructed $\Xi_{c}^{0}$ in on-peak data. The distribution for the $\Xi_{c}^{0}$ signal region (see text). is plotted as black points in (a) for the $\Xi^{-} \pi^{+}$ mode. The $p^{*}$ distribution in sidebands is also shown as green/light points. and are fitted to a smooth curve, superimposed on the data. The sideband-subtracted $p^{*}$ distributions are shown in (b). The lower peak below $p^{*}<1.5 \mathrm{GeV} / \mathrm{c}$ is primarily from the $\Xi_{c}^{0}$ production from $\mathrm{B} / \overline{\mathrm{B}}$ decays. 


\subsubsection{2 $\Xi_{c}^{0} \rightarrow \Lambda^{0} K^{-} \pi^{+}$reconstruction and selection}

A $\Lambda^{0}$ candidate is combined with two oppositely charged tracks, one identified as a Kaon (KLHTight), the other assigned a pion mass, to form a $\Xi_{c}^{0}$ candidate. We require the $\Xi_{c}^{0}$ vertex fit to have $P\left(\chi^{2}\right)>0.01 \%$, and the signed transverse decay length (the sign is that of the cosine of the angle between the transverse displacement vector and transverse momentum vector of the $\Lambda^{0}$ candidate) of the $\Lambda^{0}$ from the primary vertex to be more than $0.15 \mathrm{~cm}$. Figure 6.6 shows the invariant mass distribution of $\Lambda^{0} K^{-} \pi^{+}$from $59 \mathrm{fb}^{-1}$ of on-peak data (left) and from the truth-matched signals (see later; essentially matched as generated MC) from MC(right). Figure 6.7 shows
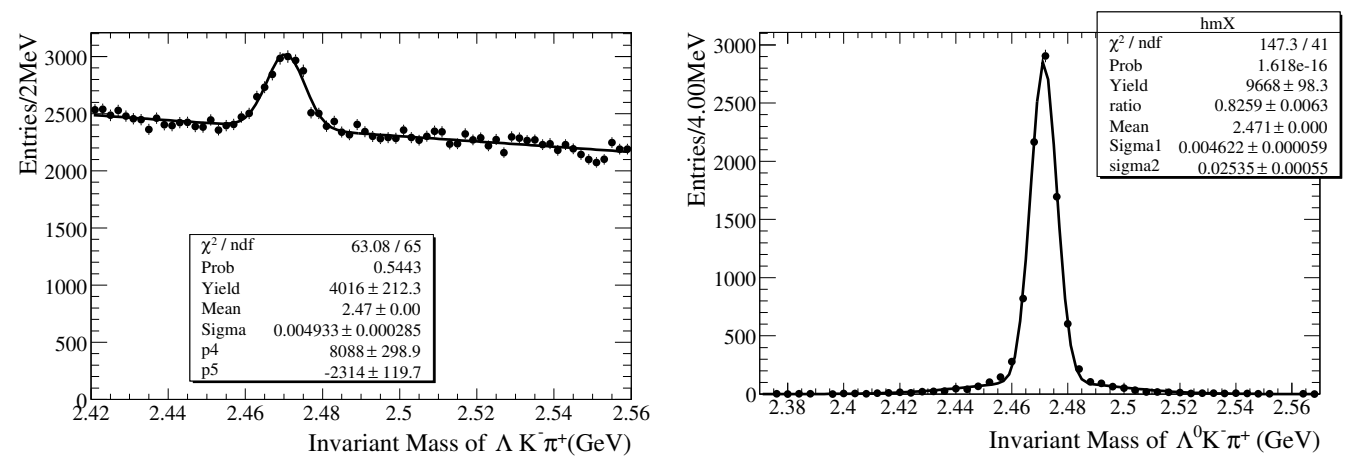

Figure 6.6: Invariant mass distribution of $\Xi_{c}^{0} \rightarrow \Lambda^{0} K^{-} \pi^{+}$candidates from the BABAR data (left), and from truth-matched signal MC (right). On the left, the signal is fitted with a single Gaussian; the fitted mean is $2470.4 \pm 0.3 \mathrm{MeV}$ with $\sigma=4.93 \pm 0.29 \mathrm{MeV}$. On the right, the signal is fitted with a double-Gaussian; the fitted mean $=2471.41 \pm$ $0.06 \mathrm{MeV}$, core Gaussian $\sigma_{1}=4.62 \pm 0.06 \mathrm{MeV}$ with $82.6 \pm 0.6 \%$ of the yield, and $\sigma_{2}=25.35 \pm 0.55 \mathrm{MeV}$.

the $\Xi_{c}^{0} \rightarrow \Lambda^{0} K^{-} \pi^{+}$candidates with $p^{*}>2.2 \mathrm{GeV} / c$. A broad structure is observed 
below the $\Xi_{c}^{0}$ mass between 2.33 and $2.43 \mathrm{GeV}$. This is supposed to be a reflection from the reaction $\Xi_{c}^{0} \rightarrow \Sigma^{0} K^{-} \pi^{+}$, where the $\gamma$ daughter of $\Sigma^{0}$ is not included in the $\Xi_{c}^{0}$ reconstruction. To study this structure further, Monte Carlo events ( $c \bar{c}$ generic) and off-peak data are used to reconstruct the $\Xi_{c}^{0} \rightarrow \Sigma^{0} K^{-} \pi^{+}$decays by vertexing the $\Lambda^{0}, \gamma, K^{-}$, and $\pi^{-}$. Figure 6.8(a) and (b) show the invariant mass distributions from the Monte Carlo and the data in black curves, respectively. The truth-matched reconstructed events from the Monte Carlo, shown in red, peak at the proper $\Xi_{c}^{0}$ value. To reproduce the structure (from reflection), we take the same $\Xi_{c}^{0} \rightarrow \Sigma^{0} K^{-} \pi^{+}$decays and plot the $\Lambda K^{-} \pi^{+}$invariant mass. This spectrum is shown in the blue dashed histogram, and the subset of the MC events with the $\Xi_{c}^{0} \rightarrow \Sigma^{0} K^{-} \pi^{+}$truth-matched is shown in a pink, hatched histogram. A similar broad structure is observed in the off-peak invariant mass distribution, if the $\gamma$ is dropped and only the $\Lambda^{0}, K^{-}$, and $\pi^{-}$ from $\Xi_{c}^{0} \rightarrow \Sigma^{0} K^{-} \pi^{+}$are combined. However, without any $p^{*}$ cut this broad structure cannot be observed because of the presence of a very high background. Since the primary analysis does not use any $p^{*}$ cut and a $\Xi_{c}^{0}$ invariant mass requirement of $>2.45 \mathrm{GeV}$ is applied, this reflection does not affect the results.

\subsection{3 $\Xi_{c}^{+}$Selection}

The $\Xi_{c}^{+}$candidates are reconstructed in the $\Xi^{-} \pi^{+} \pi^{+}$final state. Since the main difference from the $\Xi_{c}^{0} \rightarrow \Xi^{-} \pi^{+}$final state is an extra pion, the same selection criteria are applied. The reconstructed mass distributions of $\Xi_{c}^{+}$candidates are shown in Figures 6.9(a) and (b) from the data and signal MC, respectively. The data sample 


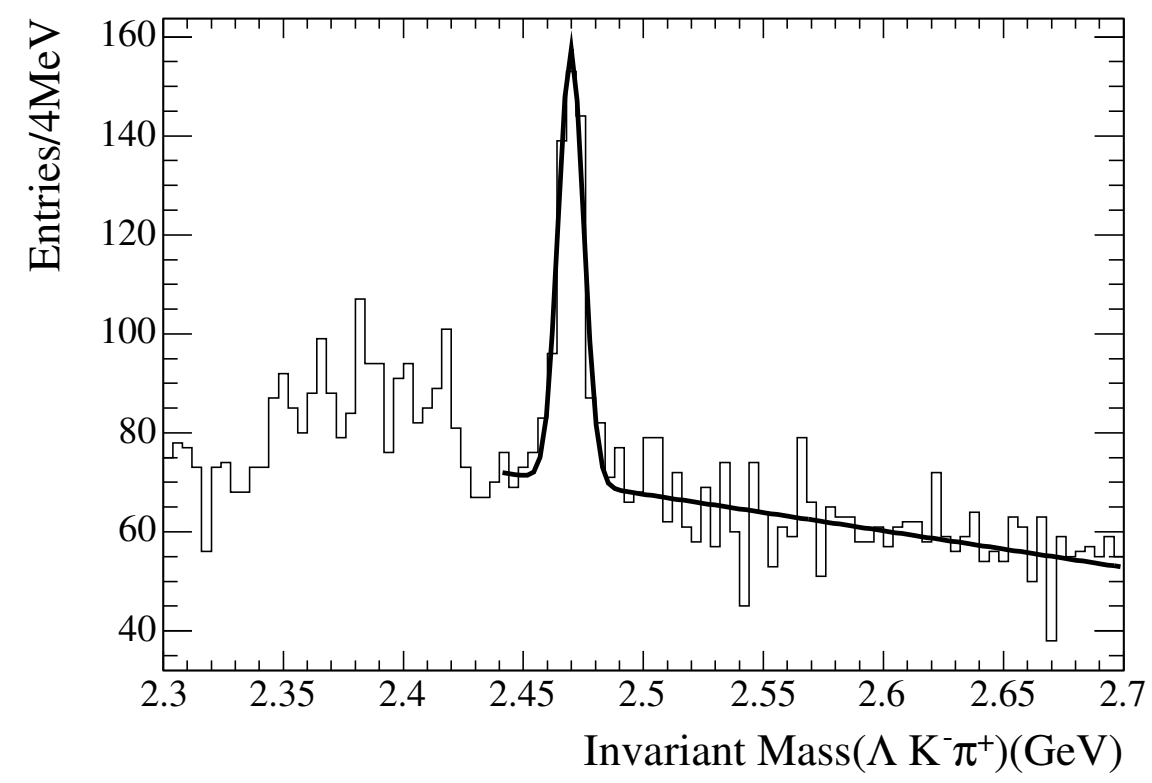

Figure 6.7: Invariant mass distribution of the $\Xi_{c}^{0} \rightarrow \Lambda^{0} K^{-} \pi^{+}$in the BABAR data, where a center of mass momentum $p^{*}>2.2 \mathrm{GeV}$ cut is made.

used for the mass spectrum in figure 6.9 (a) is $99 \mathrm{fb}^{-1}$.

\subsubsection{Reconstruction and selection of $\Lambda_{c}^{+}$}

The $\Lambda_{c}^{+}$candidates are reconstructed in their $p K^{-} \pi^{+}, p K_{S}^{0}$, and $\Lambda^{0} \pi^{+}$final states from the reskimmed dataset described in Sec 5.3.2.4. The corresponding Branching fractions are listed in the Table 6.1 from Partcil Data Group (PDG) 2006. Figures $6.10,6.11$ and 6.13 show the invariant mass distribution of $\Lambda_{c}^{+}$candidates in $59 \mathrm{fb}^{-1}$ of on-peak data in the $p K^{-} \pi^{+}, p K_{S}^{0}$, and $\Lambda^{0} \pi^{+}$final states, respectively. 

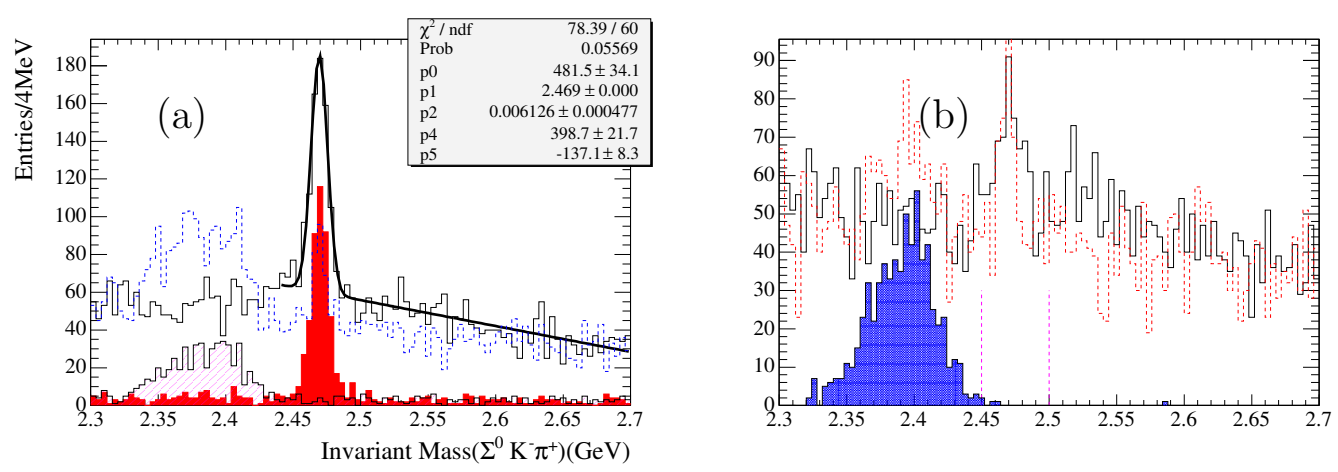

Figure 6.8: Invariant mass distribution of $\Xi_{c}^{0} \rightarrow \Sigma^{0} K^{-} \pi^{+}$candidate in the $c \bar{c}$ generic MC (a) and the BABAR off-peak data (b). (a), the invariant mass of $\Lambda^{0} \gamma K^{-} \pi^{-}$is shown as black line with fit curve, the invariant mass of $\Lambda^{0} K^{-} \pi^{-}$is shown as blue line, truth matched $\Xi_{c}^{0}$ are shown as red filled area, and truth matched $\Xi_{c}^{0}$ with the $\gamma$ dropped are shown as pink hatched area); (b) the invariant mass of $\Lambda^{0} \gamma K^{-} \pi^{-}$is shown as black line, the invariant mass of $\Lambda^{0} K^{-} \pi^{-}$is shown as red line. The blue solid histogram shows the $\left(\Lambda K^{-} \pi^{+}\right)$invariant mass for candidates with $\left(\Lambda \gamma K^{-} \pi^{+}\right)$ invariant mass in the range of $(2.47,2.5) \mathrm{GeV} / c^{2}$.

Table 6.1: The branching fractions of the $\Lambda_{c}^{+}$decay modes [PDG 2006]

\begin{tabular}{ccc}
\hline \hline Decay mode & $\mathcal{B}$ & $\frac{\mathcal{B}}{\mathcal{B}\left(\Lambda_{c}^{+} \rightarrow p^{+} K^{-} \pi^{+}\right)}$ \\
\hline$\Lambda_{c}^{+} \rightarrow p^{+} K^{-} \pi^{+}$ & $(5.0 \pm 1.3) \%$ & 1.0 \\
$\Lambda_{c}^{+} \rightarrow p^{+} \bar{K}^{0}$ & $(2.3 \pm 0.6) \%$ & $0.47 \pm 0.04$ \\
$\Lambda_{c}^{+} \rightarrow \Lambda^{0} \pi^{+}$ & $(0.90 \pm 0.28) \%$ & $0.180 \pm 0.032$ \\
Total & $(8.2 \pm 1.5) \%$ & $1.65 \pm 0.05$ \\
\hline
\end{tabular}

6.1.4.1 Reconstruction and selection of $\Lambda_{c}^{+} \rightarrow p^{+} K^{-} \pi^{+}$

We combine three charged tracks, one identified as a proton (pLHVeryLoose), an oppositely charged track identified as a kaon (KLHtight), and the third one (of the same charge as the proton) with a pion mass assigned. The tracks are fitted to a 

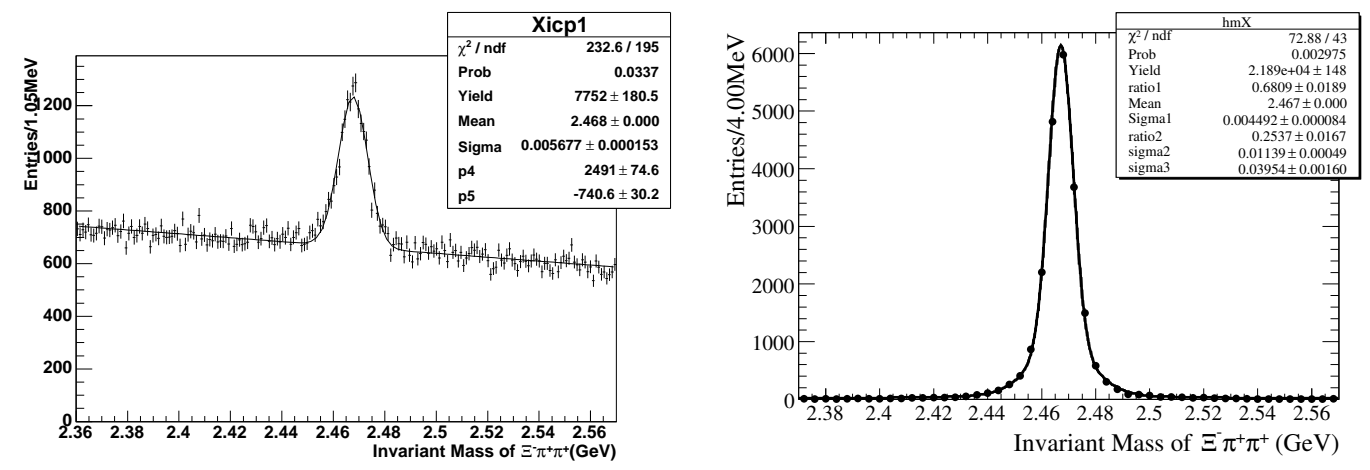

Figure 6.9: Invariant mass distribution of $\Xi_{c}^{+} \rightarrow \Xi^{-} \pi^{+} \pi^{+}$candidates from the BABAR data on the left, and from truth-matched signal $\mathrm{MC}$ on the right. On the left, the signal is fitted with a single Gaussian; the fitted mean is $2467.68 \pm 0.14 \mathrm{MeV}$ with $\sigma=5.68 \pm 0.15 \mathrm{MeV}$. On the right, the signal is fitted with a triple-Gaussian;the fitted parameters are: mean $=2467.15 \pm 0.04 \mathrm{GeV}$, core Gaussian $\sigma_{1}=4.49 \pm 0.08 \mathrm{MeV}$ with $68.09 \pm 1.89 \%$ of the yield, $\sigma_{2}=11.39 \pm 0.49 \mathrm{MeV}$ with $25.37 \pm 1.67$ of the yield, and $\sigma_{3}=39.54 \pm 0.16 \mathrm{MeV}$.

vertex, in order to reconstruct a $\Lambda_{c}^{+}$candidate. Figures 6.10 show the invariant mass distribution of $\Lambda_{c}^{+}$candidates from $99 \mathrm{fb}^{-1}$ of data (left) and truth-matched signal $\mathrm{MC}$ (right). The mass distribution in the data is fitted to a double Gaussian (for the signal); the effective resolution $\sigma_{\text {eff }}$ of mass distribution is calculated as $6.0 \mathrm{MeV}$. The fitted mean of the peak is $2285.89 \pm 0.06 \mathrm{MeV}$.

\subsubsection{Reconstruction and selection of $\Lambda_{c}^{+} \rightarrow p^{+} K_{S}^{0}$}

The $\Lambda_{c}^{+}$candidates are reconstructed by vertexing a proton (pLHVeryLoose) with a $K_{s}^{0}$ meson using the TreeFitter vertex package. The $K_{S}^{0}$ reconstruction and selection are described in Section 4.2.1. Figure 6.11 shows the invariant mass distribution of $\Lambda_{c}^{+} \rightarrow p^{+} K_{S}^{0}$ candidates in $99 \mathrm{fb}^{-1}$ of data (left) and in truth-matched 

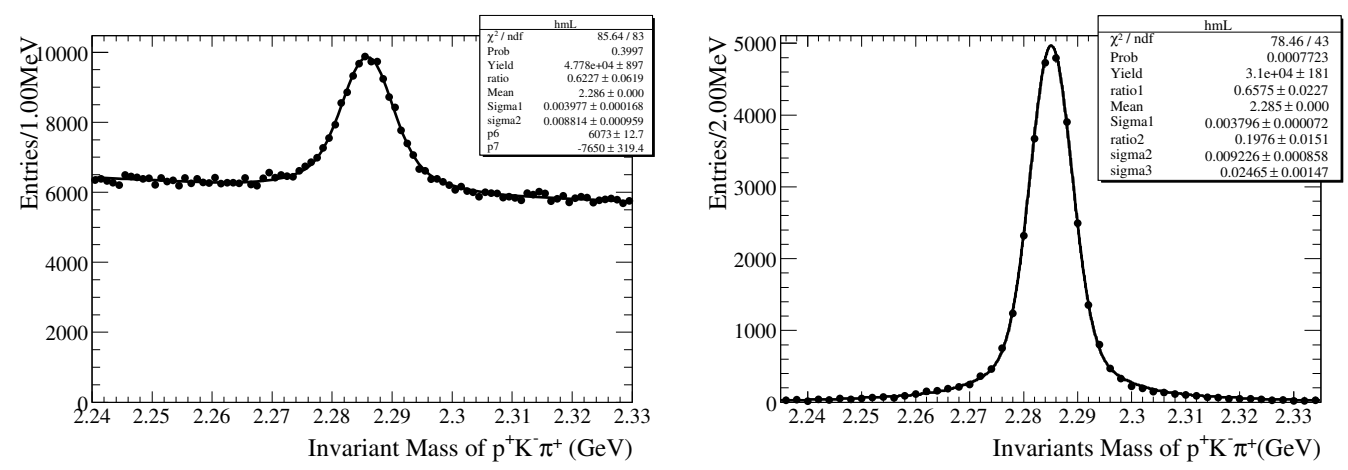

Figure 6.10: Invariant mass distribution of $\Lambda_{c}^{+} \rightarrow p^{+} K^{-} \pi^{+}$candidates in the BABAR data (left) and in truth-matched signal Monte Carlo (right). On the left, the signal is fitted to a double-Gaussian with common mean. The Gaussian mean is $2285.88 \pm$ $0.06 \mathrm{MeV}, \sigma_{1}=4.0 \pm 0.2 \mathrm{MeV}$ with a yield fraction of $62.3 \%$, and $\sigma_{2}$ is $8.86 \pm 0.96 \mathrm{MeV}$. On the right, the signal is fitted to a triple-Gaussian, with mean $2285.11 \pm 0.03 \mathrm{MeV}$, $\sigma_{1}=3.80 \pm 0.07 \mathrm{MeV}$ with a yield of fraction of $66 \%, \sigma_{2}=9.22 \pm 0.85 \mathrm{MeV}$ with a yield fraction of $20 \%$ and $\sigma_{3}=24.65 \pm 1.47 \mathrm{MeV}$.

signal MC (right). The signal shape in the data is fitted to a single Gaussian with a $\sigma$ of $5.8 \pm 0.1 \mathrm{MeV}$.

\subsubsection{Reconstruction of $\Lambda_{c}^{+} \rightarrow \Lambda^{0} \pi^{+}$}

In this case, the $\Lambda_{c}^{+}$is reconstructed by vertexing a $\Lambda^{0}$ and a $\pi^{-}$by using the TreeFitter. The reconstructed invariant mass distributions of $\Lambda_{c}^{+} \rightarrow \Lambda^{0} \pi^{+}$candidates are shown in Figure 6.13 (the left one is built from $99 \mathrm{fb}^{-1}$ of data and the right one is from truth-matched signal MC). The signal shape is fitted with a single Gaussian with a $\sigma=5.26 \pm 0.20 \mathrm{MeV}$ in the data.

In a previous study of $\Lambda_{c}^{+} \rightarrow \Lambda^{0} \pi^{+}$[49] by $B A B A R$, it was shown that there are two sources contributing broadly under the signal (called peaking backgrounds): 

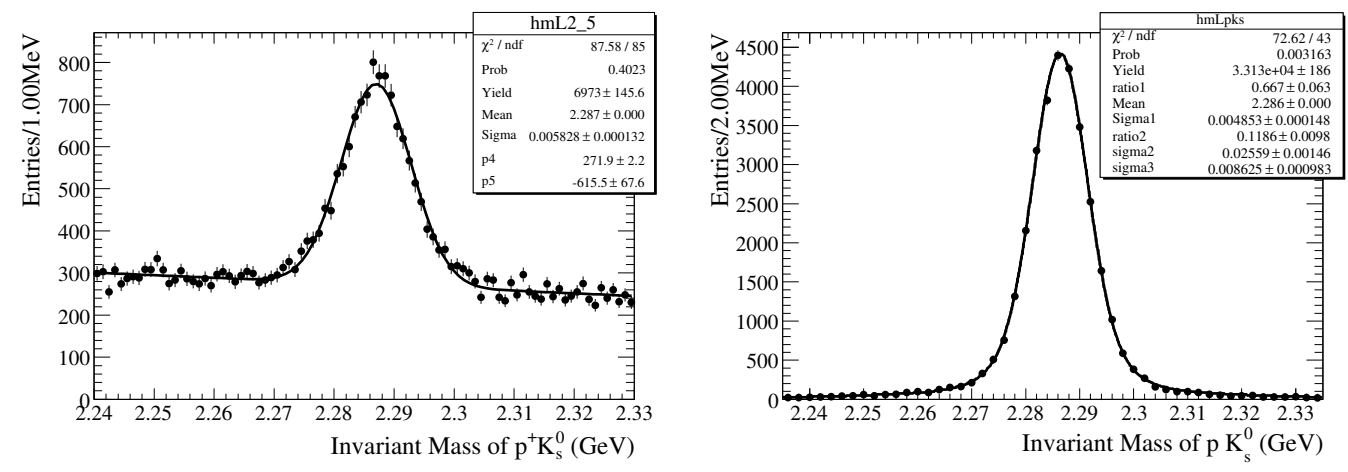

Figure 6.11: Invariant mass distribution of $\Lambda_{c}^{+} \rightarrow p^{+} K_{S}^{0}$ candidates in the BABAR data (left) and in truth-matched signal Monte Carlo (right). On the left, the signal is fitted to a single Gaussian on linear background. The Gaussian mean is $2287.02 \pm 0.12 \mathrm{MeV}$ with $\sigma=5.83 \pm 0.13 \mathrm{MeV}$. On the right, the signal is fitted to a triple-Gaussian, with mean $2286.41 \pm 0.04, \sigma_{1}=4.85 \pm 0.14 \mathrm{MeV}$ with a yield fraction of $67 \%, \sigma_{2}=$ $25.59 \pm 1.46 \mathrm{MeV}$ with a yield fraction of $12 \%$ and $\sigma_{3}=8.63 \pm 0.98 \mathrm{MeV}$.

one is from $\Xi_{c}^{0} \rightarrow \Xi^{-} \pi^{+}$where the the $\pi^{-}$daughter of the $\Xi^{-}$is missed (centered at $\left.2.3 \mathrm{GeV} / c^{2}\right)$ and one is from $\Lambda_{c}^{+} \rightarrow \Sigma^{0} \pi^{+}$where the daughter $\gamma$ of the $\Sigma^{0}$ is missed (centered at $2.2 \mathrm{GeV} / c^{2}$ ). We reconstruct $\Lambda_{c}^{+} \rightarrow \Lambda^{0} \pi^{+}$candidates from $18 \mathrm{fb}^{-1}$ of data. The mass spectra are shown for $p^{*}<2.0 \mathrm{GeV} / c$ in Fig $6.12(\mathrm{a})$, and for $p^{*}>2.0 \mathrm{GeV} / c$ in Fig 6.12(b). The structures from reflection are observed for $p^{*}>2.0 \mathrm{GeV} / c$; but none such is visible above the background for $p^{*}<2.0 \mathrm{GeV} / c$ where most of the signals from $B$ decays are expected. We therefore neglect these reflections in this study.

\section{2 $B$ meson reconstruction and selection}

The reconstruction of the charmed baryon candidates: $\Xi_{c}^{+}, \Xi_{c}^{0}$ and $\Lambda_{c}^{+}$are described in the previous section. $B$ candidates are formed in events with at least one 

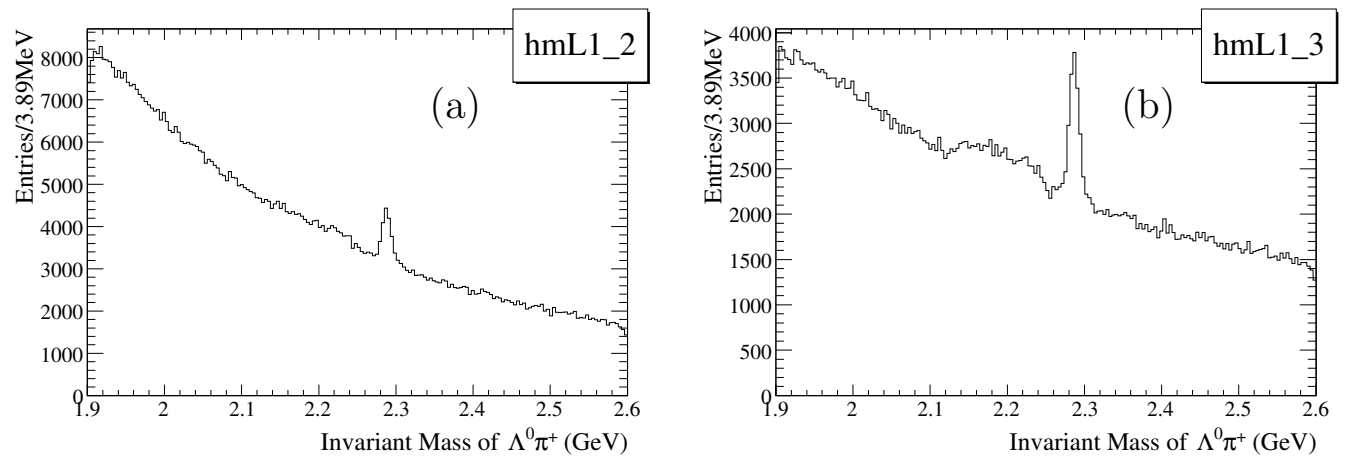

Figure 6.12: Invariant mass distribution of $\Lambda_{c}^{+} \rightarrow \Lambda^{0} \pi^{+}$from $18 \mathrm{fb}^{-1}$ of data: (a) $p^{*}<2 \mathrm{GeV}$, and (b) $p^{*}>2 \mathrm{GeV}$.
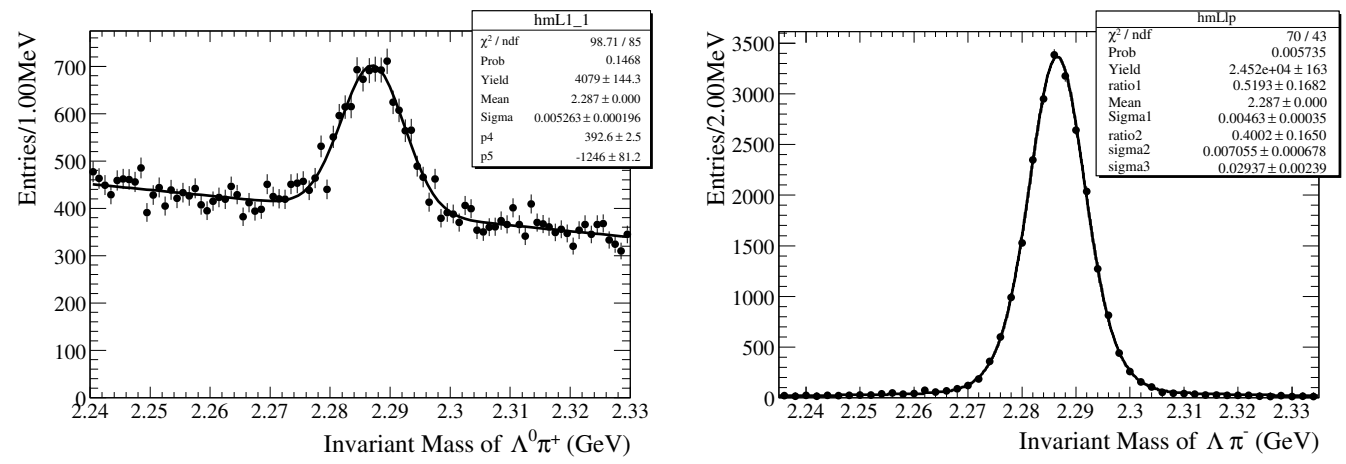

Figure 6.13: Invariant mass distribution of $\Lambda_{c}^{+} \rightarrow \Lambda^{0} \pi^{+}$in the BABAR data (left) and in truth-matched Monte Carlo (right). On the left, the signal is fitted to a single Gaussian on a linear background. The fitted mean is $2287.13 \pm 0.19 \mathrm{MeV}$ with $\sigma=5.26 \pm 0.20 \mathrm{MeV}$. On the right, the signal is fitted to a triple-Gaussian, with mean $=2286.53 \pm 0.04 \mathrm{MeV}, \sigma_{1}=4.63 \pm 0.35 \mathrm{MeV}$ with a yield fraction of $52 \%$, $\sigma_{2}=7.06 \pm 0.66 \mathrm{MeV}$ with a yield fraction of $40 \%$ and $\sigma_{3}=29.37 \pm 2.39 \mathrm{MeV}$. 
charmed baryon and one charmed anti-baryon candidate which do not overlap (share any tracks) in the following decay final states: $B^{-} \rightarrow \Xi_{c}^{0} \bar{\Lambda}_{c}^{-}, \bar{B}^{0} \rightarrow \Xi_{c}^{+} \bar{\Lambda}_{c}^{-}, B^{-} \rightarrow$ $\Lambda_{c}^{+} \bar{\Lambda}_{c}^{-} K^{-}$and $\bar{B}^{0} \rightarrow \Lambda_{c}^{+} \bar{\Lambda}_{c}^{-} K_{s}^{0}$. One of the two charmed baryons is reconstructed in the $\Lambda_{c}^{+} \rightarrow p^{+} K^{-} \pi^{+}$final state (as this has a much higher efficiency and branching fraction). We then apply further selection cuts, described in Sections 6.2.1 and 6.2.4. In events with more than one reconstructed $B$ candidates, we select a single one as described in Section 6.2.3, discarding the rest. Finally, an unbinned maximum likelihood fit is performed on the $m_{\mathrm{ES}}$ (defined below) distribution to extract the signal yield and calculate the branching fractions.

\subsection{1 $m_{\mathrm{ES}}$ and $\Delta E$ variables}

The correctly reconstructed $B$ meson candidates should have an invariant mass equal to the $B$ meson mass within errors and energy equal to half of the CM energy. The $B$ mass resolution is improved in general by taking advantage of the kinematic constraints of the $e^{+} e^{-} \rightarrow \Upsilon(4 S) \rightarrow B \bar{B}$ process to produce the kinematic variables: $m_{\mathrm{ES}}$ and $\Delta E$. The beam energy constrained mass is defined as

$$
\begin{aligned}
m_{\mathrm{ES}} & =\sqrt{s / 4-p_{B}^{* 2}} \\
& =\sqrt{\left(\frac{s+2 \vec{p}_{i} \cdot \vec{p}_{B}}{2 E_{i}}\right)^{2}-p_{B}^{2}}
\end{aligned}
$$

in both $e^{+} e^{-}$rest frame and lab frame; and the other variable, $\Delta E$ is the difference between the reconstructed $B$ energy and the beam energy in CM frame, defined as

$$
\Delta E=E_{B}^{*}-\sqrt{s} / 2
$$


where $\sqrt{s}$ is the total center-of-mass energy of $e^{+} e^{-}$collision; $\vec{p}_{i}$ and $E_{i}$ are the 3 -momentum and the total energy of the $\Upsilon(4 S)$ in the lab frame; $\vec{p}_{B}$ is the three momentum of $B$ candidate in the lab frame; and $p_{B}^{*}$ and $E_{B}^{*}$ are the momentum and energy of $B$ candidate in the $e^{+} e^{-}$rest frame. In the above equations, the energy of the $B$ meson is replaced by half of the beam energy in the $m_{\mathrm{ES}}$ and $\Delta E$ calculation, because the uncertainty on the beam energy is much smaller than that of the energy of the reconstructed $B$. For signal events, $m_{\mathrm{ES}} \sim m_{B}$ and $\Delta E \sim 0$.

The resolution of $m_{\mathrm{ES}}$ is determined by the beam energy when calculated in the lab frame without any knowledge of the masses of the $B$ daughters, and is largely mode-independent. The resolution of the $\Delta E$ distribution reflects the resolution of the CM energy of the reconstructed $B$, and is generally dominated by the detector resolution.

Two areas, illustrated in Fig 6.14, are defined in the $\left(m_{\mathrm{ES}}, \Delta E\right)$ plane to determine the signal yield over the background: the signal region and the region for background study. In a two dimensional display of the reconstructed mass of the $B$ candidates vs. the energy difference between that of the reconstructed $B$ candidates and half of the CM energy, the signal events should congregate in a box around the $B$ mass and zero energy difference; Region I is the signal region and region II and III are the background study region, defined as sideband regions; these are shown below:

- signal region: $5.27<m_{\mathrm{ES}}<5.29 \mathrm{GeV}$ and $|\Delta E|<3 \sigma(\Delta E)$

- $m_{\mathrm{ES}}$ sideband: $5.20<m_{\mathrm{ES}}<5.26 \mathrm{GeV}$ and $|\Delta E|<0.2 \mathrm{GeV}$ 


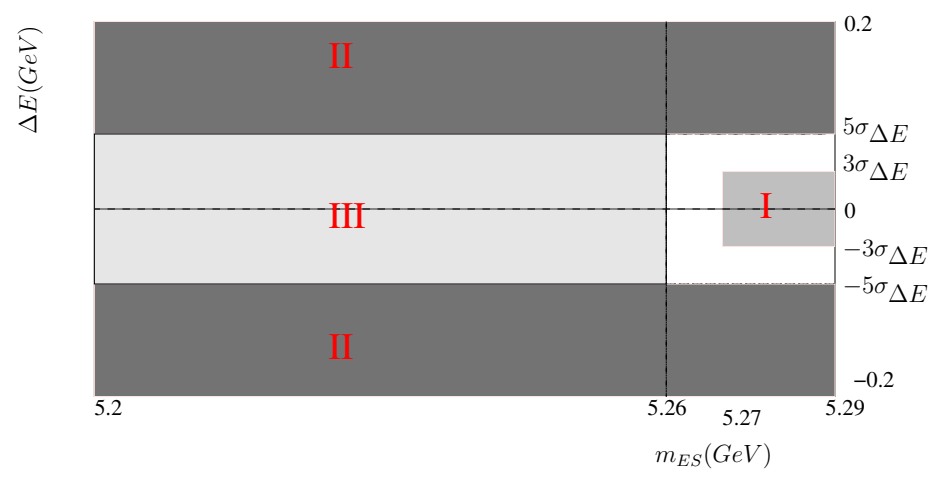

Figure 6.14: The $m_{\mathrm{ES}}-\Delta E$ region used to define signal and background regions.

- $\Delta E$ side band: $5.2<m_{\mathrm{ES}}>5.29 \mathrm{GeV}$ and $|\Delta E|>5 \sigma(\Delta E)$; for the $B \rightarrow \Xi_{c} \bar{\Lambda}_{c}^{-}$ mode, the reflection backgrounds are peaking in the region $\Delta E<-0.05 \mathrm{GeV}$.

\subsubsection{Signal shape in Monte Carlo}

The $B$ signal shapes are derived from signal $\mathrm{MC}$ in the modes shown in Table 1.6. The combined mass of a $\Xi_{c}$ and a $\Lambda_{c}^{+}, m_{\Xi_{c}^{0}}+m_{\Lambda_{c}^{+}}=4.765 \mathrm{GeV}$, and the combined mass of a $\Lambda_{c}^{+}$and a $\bar{\Lambda}_{c}^{-} m_{\Lambda_{c}^{+}}+m_{\bar{\Lambda}_{c}^{-}}=4.70 \mathrm{GeV}$; implying that more than $85 \%$ of the phase space in $B$ decay is used up by the charmed baryon masses. So, when the invariant masses of the charmed baryons are constrained to their nominal values (from $\mathrm{PDG}$ ), the $B$ mass resolution is no longer dominated by the detector resolution but by the beam energy resolution.

Figure $6.15,6.16$ and 6.17 show $m_{\mathrm{ES}}$ and $\Delta E$ distributions from the $B^{-} \rightarrow$ $\Lambda_{c}^{+} \bar{\Lambda}_{c}^{-} K^{-}$decay, where in (a) both $\Lambda_{c}^{+} \rightarrow p K \pi$ the invariant masses of both $\Lambda_{c}^{+}$are constrained to the nominal masses and in (b), neither of the $\Lambda_{c}^{+}$invariant mass is 
constrained. The benefit of the mass constraint can be observed in the significant improvement of the resolution in $\Delta E$. The $\Delta E$ distributions reconstructed from the (centrally produced) signal MC, shown in Figure 6.17, are fitted with a double Gaussian function, where the $\sigma$ of the core Gaussian of $\Delta E$ is seen to improve from $8 \mathrm{MeV}$ to $4 \mathrm{MeV}$ as a result of constraining the $\Lambda_{c}^{+}$mass. The resolutions of the $m_{\mathrm{ES}}$ distributions remain almost the same in both cases as shown in Figure 6.16 in which the $m_{\mathrm{ES}}$ distributions are fitted with a single Gaussian for the signal ( $\sigma=2.55$ and $2.53 \mathrm{MeV}$ in (a) and (b), respectively) and the Argus function (7.2.1) for the background. But as illustrated in Figures 6.15 (a) and (b), the correlation between $m_{\mathrm{ES}}$ and $\Delta E$ is more significant after the mass constraint is applied to the $\Lambda_{c}^{+}$mass, since the $\Delta E$ resolution is also dominated by the beam energy spread now, rather than the detector resolution.

Table 6.2 summarizes the resolutions in $m_{\mathrm{ES}}$ and $\Delta E$ for all the decay modes obtained from the simulation after applying the mass constraint to all the charmed baryons. The $m_{\mathrm{ES}}$ resolution is dominated by the beam energy and essentially identical for all final states. The signal distributions in $\Delta E$ are fitted with a double Gaussian shape with the core Gaussian being dominant in all modes. The fraction of events in the core Gaussian is between $85 \sim 89 \%$ for the five charged three-body decay modes, $96 \sim 98 \%$ for the neutral three-body mode and all the two-body modes. The width of the core Gaussian is about $3.9 \mathrm{MeV}$ for all the three-body modes, and is about $5 \mathrm{MeV}$ for all the two-body modes. The $\sigma_{\text {eff }}$ is approximately $7.5 \mathrm{MeV}$ for all of the $\Lambda_{c}^{+} \bar{\Lambda}_{c}^{-} K^{-}$modes, $4.6 \mathrm{MeV}$ for the $\Lambda_{c}^{+} \bar{\Lambda}_{c}^{-} K_{S}^{0}$ mode, and $6.0 \mathrm{MeV}$ for all of 

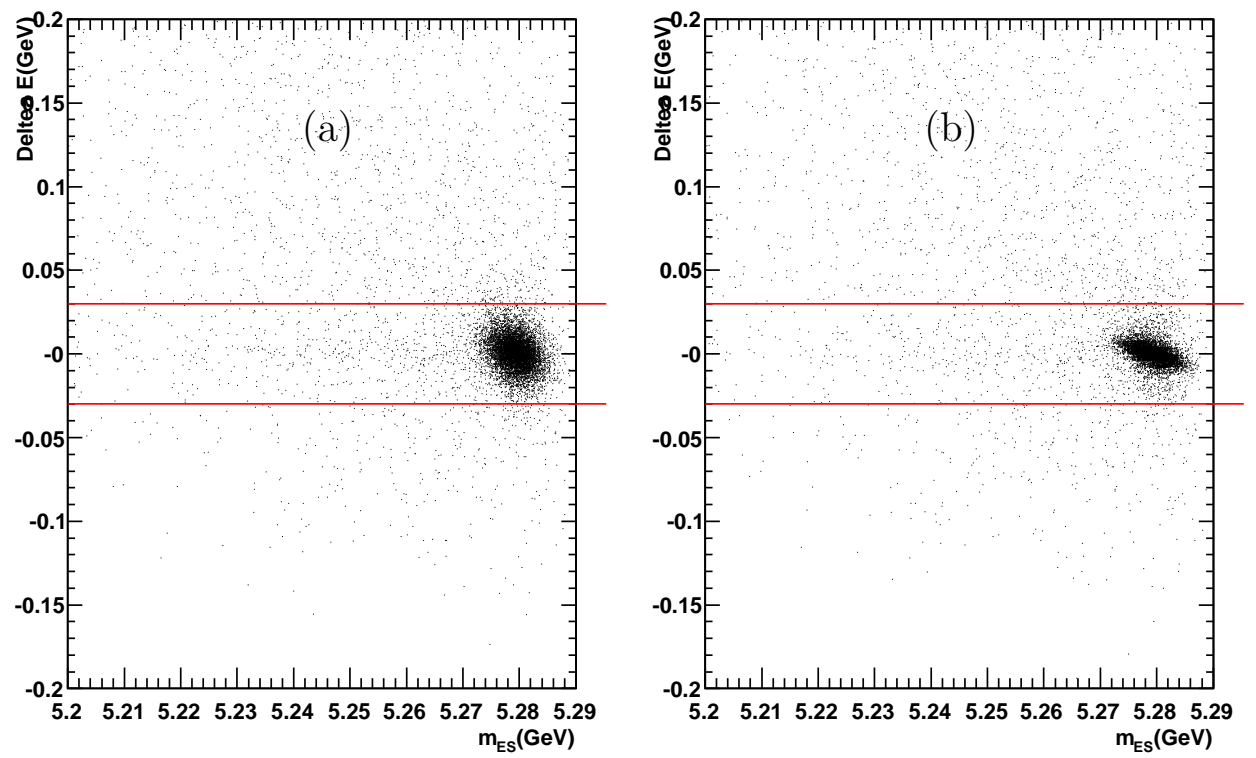

Figure 6.15: $\Delta E$ vs. $m_{\mathrm{ES}}$ for a signal MC sample of $B^{-} \rightarrow \Lambda_{c}^{+} \bar{\Lambda}_{c}^{-} K^{-}$, and both $\Lambda_{c}^{+}$decay to $p K \pi$ unconstrained (left) and constrained (right) $\Lambda_{c}^{+}$candidates masses, passed all the selection criteria.
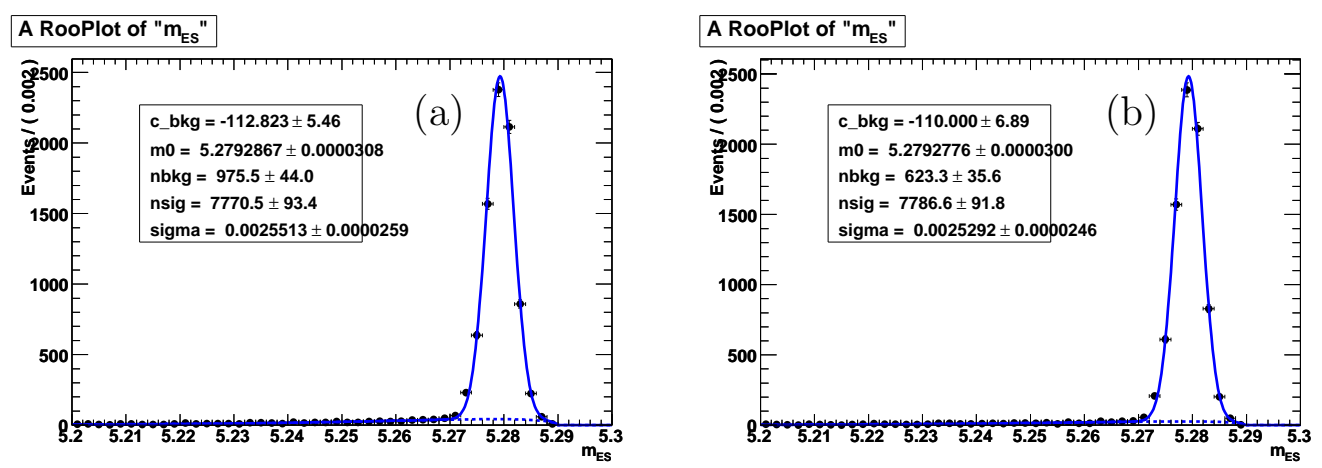

Figure 6.16: $m_{\mathrm{ES}}$ for a signal MC sample of $B^{-} \rightarrow \Lambda_{c}^{+} \bar{\Lambda}_{c}^{-} K^{-}$, and both $\Lambda_{c}^{+}$decay to $p K \pi$ unconstrained (left) and constrained (right) $\Lambda_{c}^{+}$candidates masses, passed all the selection criteria. 

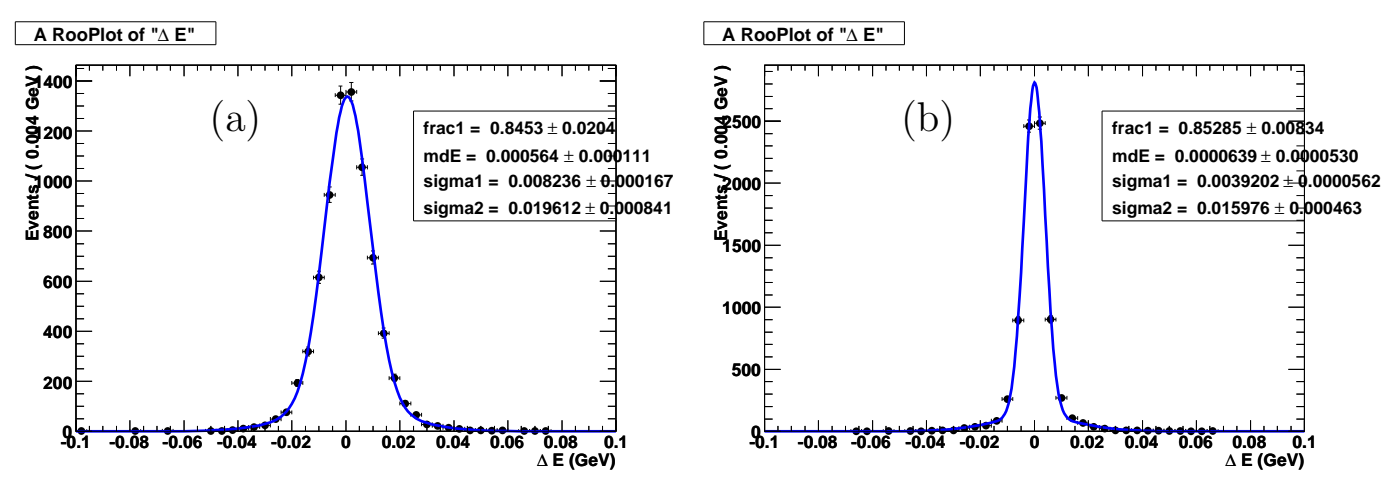

Figure 6.17: $\Delta E$ for a signal MC sample of $B^{-} \rightarrow \Lambda_{c}^{+} \bar{\Lambda}_{c}^{-} K^{-}$, and both $\Lambda_{c}^{+}$decay to $p K \pi$ unconstrained (left) and constrained (right) $\Lambda_{c}^{+}$candidates masses, passed all the selection criteria. Truth matching are applied here.

the $\Xi_{c} \bar{\Lambda}_{c}^{-}$modes. This difference in the resolution is expected, since more of the $B$ meson energies are used up in the three-body decays than in the two-body decays.

\subsubsection{Choice of best candidate selection}

At times multiple combinations satisfy the selection criteria in the same event in the reconstruction of $B$ candidates. The multiplicity of the $B$ candidates depends on the decay mode and the selection cuts. On average, we get $1 \sim 1.25 B$ Candidates per event in the signal region defined in Section 6.2.1 in both data and signal MC for different decay channels. . The events with multiple candidates are categorized as follows (the decay $B^{-} \rightarrow \Lambda_{c}^{+} \bar{\Lambda}_{c}^{-} K^{-}$, where $\Lambda_{c}^{+} \rightarrow p^{+} K^{-} \pi^{+}$for both $\Lambda_{c}$ is used as an example,and only the dominant cases are listed):

- The same final-state particles are present, but two of the tracks are swapped (most commonly the $K^{-}$daughter of the $\Lambda_{c}^{+}$and the $K^{-}$bachelor). 
Table 6.2: $m_{\mathrm{ES}}$ and $\Delta E$ resolution

\begin{tabular}{|c|c|c|c|c|c|}
\hline \multirow[t]{2}{*}{ Decay mode } & \multirow{2}{*}{$\begin{array}{c}m_{\mathrm{ES}} \\
\sigma \\
(\mathrm{MeV})\end{array}$} & \multicolumn{4}{|c|}{$\Delta E$} \\
\hline & & $\begin{array}{c}\sigma_{1} \\
(\mathrm{MeV})\end{array}$ & $f_{1}$ & $\begin{array}{c}\sigma_{2} \\
(\mathrm{MeV})\end{array}$ & $\begin{array}{c}\text { RMS } \\
(\mathrm{MeV})\end{array}$ \\
\hline \multicolumn{6}{|l|}{$B^{-} \rightarrow \Lambda_{c}^{+} \bar{\Lambda}_{c}^{-} K^{-}$} \\
\hline$\Lambda_{c}^{+} \rightarrow p^{+} K^{-} \pi^{+}, \bar{\Lambda}_{c}^{-} \rightarrow p^{-} K^{+} \pi^{-}$ & $2.53 \pm 0.02$ & $3.92 \pm 0.06$ & $0.85 \pm 0.01$ & $15.98 \pm 0.46$ & 7.12 \\
\hline$\Lambda_{c}^{+} \rightarrow p^{+} K^{-} \pi^{+}, \bar{\Lambda}_{c}^{-} \rightarrow p^{-} K_{S}^{0}$ & $2.54 \pm 0.02$ & $3.96 \pm 0.05$ & $0.88 \pm 0.01$ & $19.42 \pm 0.52$ & 7.75 \\
\hline$\Lambda_{c}^{+} \rightarrow p^{+} K_{S}^{0}, \bar{\Lambda}_{c}^{-} \rightarrow p^{-} K^{+} \pi^{-}$ & $2.51 \pm 0.02$ & $3.96 \pm 0.05$ & $0.88 \pm 0.01$ & $18.92 \pm 0.51$ & 7.52 \\
\hline$\Lambda_{c}^{+} \rightarrow p^{+} K^{-} \pi^{+}, \bar{\Lambda}_{c}^{-} \rightarrow \bar{\Lambda}^{0} \pi^{-}$ & $2.56 \pm 0.02$ & $3.89 \pm 0.05$ & $0.87 \pm 0.01$ & $17.37 \pm 0.53$ & 7.32 \\
\hline$\Lambda_{c}^{+} \rightarrow \Lambda^{0} \pi^{+}, \bar{\Lambda}_{c}^{-} \rightarrow p^{-} K^{+} \pi^{-}$ & $2.53 \pm 0.02$ & $4.02 \pm 0.05$ & $0.89 \pm 0.01$ & $19.59 \pm 0.63$ & 7.62 \\
\hline $\bar{B}^{0} \rightarrow \Lambda_{c}^{+} \bar{\Lambda}_{c}^{-} K_{s}^{0}$ & $2.51 \pm 0.03$ & $3.80 \pm 0.06$ & $0.96 \pm 0.01$ & $13.29 \pm 1.07$ & 4.64 \\
\hline \multicolumn{6}{|l|}{$B^{-} \rightarrow \Xi_{c}^{0} \bar{\Lambda}_{c}^{-}$} \\
\hline$\Xi_{c}^{0} \rightarrow \Xi^{-} \pi^{+}, \bar{\Lambda}_{c}^{-} \rightarrow p^{-} K^{+} \pi^{-}$ & $2.57 \pm 0.04$ & $4.77 \pm 0.11$ & $0.96 \pm 0.01$ & $14.94 \pm 1.82$ & 5.62 \\
\hline$\Xi_{c}^{0} \rightarrow \Lambda^{0} K^{-} \pi^{+}, \bar{\Lambda}_{c}^{-} \rightarrow p^{-} K^{+} \pi^{-}$ & $2.50 \pm 0.04$ & $5.16 \pm 0.09$ & $0.98 \pm 0.01$ & $23.95 \pm 3.55$ & 6.05 \\
\hline $\bar{B}^{0} \rightarrow \Xi_{c}^{+} \bar{\Lambda}_{c}^{-}$ & $2.52 \pm 0.05$ & $4.97 \pm 0.11$ & $0.97 \pm 0.01$ & $19.02 \pm 2.54$ & 6.02 \\
\hline
\end{tabular}


- One of the final-state particles, typically a pion, is different between the candidates.

- The exact same $\Lambda_{c}^{+}$and $\bar{\Lambda}_{c}^{-}$are reconstructed, but a different $K^{ \pm}$is used to form a $B^{ \pm}$(often the two candidates have opposite charges)

These are the dominant sources of the multiple candidates. (While these sources can result in peaking background, not all peaking backgrounds are necessarily from multiple-candidates.) To reduce the systematic uncertainty from this type of peaking background, we select only one (best) candidate in one event. Four possible methods are studied for selecting the best candidate out of those passing all selection criteria including the $m_{\mathrm{ES}}$ and $\Delta E$ cuts:

1. The candidate with the minimum $|\Delta E|$ in the event $(\epsilon=6.64 \%)$.

2. The candidate with the best $\chi^{2}$ probability for the $B$ vertex fit $(\epsilon=6.50 \%)$.

3. The candidate with the best charmed baryon reconstruction, where we define $\chi_{b}^{2}=$ $\sum_{i=1}^{2} \frac{\left(m_{i}-\mu_{i}\right)^{2}}{\sigma_{i}^{2}}$, where $m_{i}$ is the reconstructed invariant mass of the $i$ th charmed baryon, $\mu_{i}$ is the fitted mean of the $i$ th charmed baryon, and $\sigma_{i}^{2}$ is the fitted width of the $i$ th charmed baryon. The candidate with the least $\chi_{b}^{2}$ is considered to be the best candidate in the event $(\epsilon=6.58 \%)$.

4. As a control study, we select one $B$ candidate at random declare as the best candidate $(\epsilon=6.45 \%)$.

No significant difference is observed in the selection efficiencies using any of the methods; hence we use the first method. This algorithm introduces no bias in 
signal extraction as the signal extraction is performed from the $m_{\mathrm{ES}}$ distribution only. The third method is not technically feasible, because later we constrain the mass of the charmed baryons.

\subsubsection{A summary of the selection of $B$ meson decays}

The TreeFitter vertexer combines $\Xi_{c} \Lambda_{c}, \Lambda_{c} \bar{\Lambda}_{c}$, where the $\Xi_{c}, \Lambda_{c}$ and $K_{S}^{0}$ pass the selection criteria described in Section . Before the final vertex fit, the TreeFitter invalidates all the previous fits, then re-fitting the complete decay tree. Mass constraints are imposed on all the intermediate particles $\left(K_{S}^{0}, \Lambda^{0}, \Xi^{-}, \Xi_{c}^{0}, \Xi_{c}^{+}\right.$and $\left.\Lambda_{c}^{+}\right)$ for the final reconstruction. ${ }^{1}$ The probability of $\chi^{2}$ of the fit is required to be greater than $0.01 \%$.

The following is a full list of all the common selection cuts used in this analysis:

- Probability $\left(\chi^{2}\right)$ of vertex fit $>0.01 \%$

- The $\Lambda_{c}^{+}, \Xi_{c}^{+}, \Xi_{c}^{0}$ must have an invariant mass with $18 \mathrm{MeV} / c^{2}$ of the central value before the mass constraint is applied. This corresponds to approximately a $3 \sigma$ mass cut. (the width between different charmed baryons is not exactly same, but we use the biggest one for our cut)

- B meson kinematic variable: $|\Delta E|<0.022 \mathrm{MeV}$

- The charged kaons for all the decays must pass the KLHTight PID criteria

\footnotetext{
${ }^{1}$ for some background studies, the charmed baryon masses were not constrained to the nominal value at the very beginning.
} 
- For $\bar{B}^{0} \rightarrow \Lambda_{c}^{+} \bar{\Lambda}_{c}^{-} K_{s}^{0}$, the $K_{S}^{0}$ must have signed transverse decay length greater than $2 \mathrm{~mm}$.

- If $>1$ candidate in the event, the candidate with the minimum $|\Delta E|$ is retained and all others are rejected.

Fig. 6.19and 6.18 show the $\Delta E$ vs. $m_{\mathrm{ES}}$ distribution reconstructed from on-peak data sample from decay channels $B^{-} \rightarrow \Lambda_{c}^{+} \bar{\Lambda}_{c}^{-} K^{-}$and $B^{-} \rightarrow \Xi_{c}^{0} \bar{\Lambda}_{c}^{-}$after applying all the selection cuts.
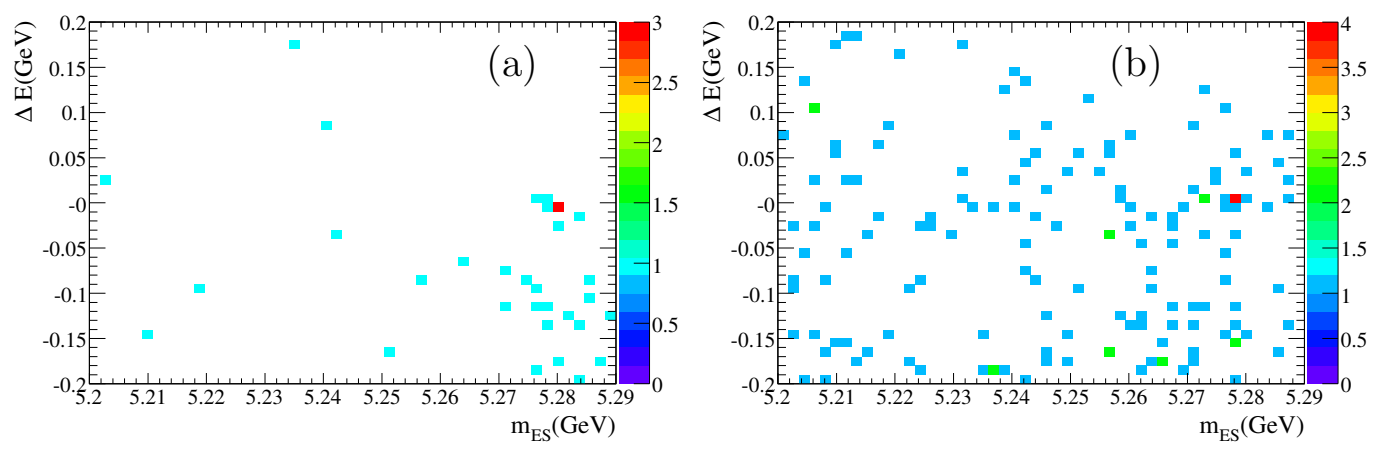

Figure 6.18: $\Delta E$ vs. $m_{\mathrm{ES}}$ distribution of $B^{-} \rightarrow \Xi_{c}^{0} \bar{\Lambda}_{c}^{-}$from on-peak dataset: (a) $\Xi_{c}^{0} \rightarrow \Xi^{-} \pi^{+}$; (b) $\Xi_{c}^{0} \rightarrow \Lambda^{0} K^{-} \pi^{+}$. 

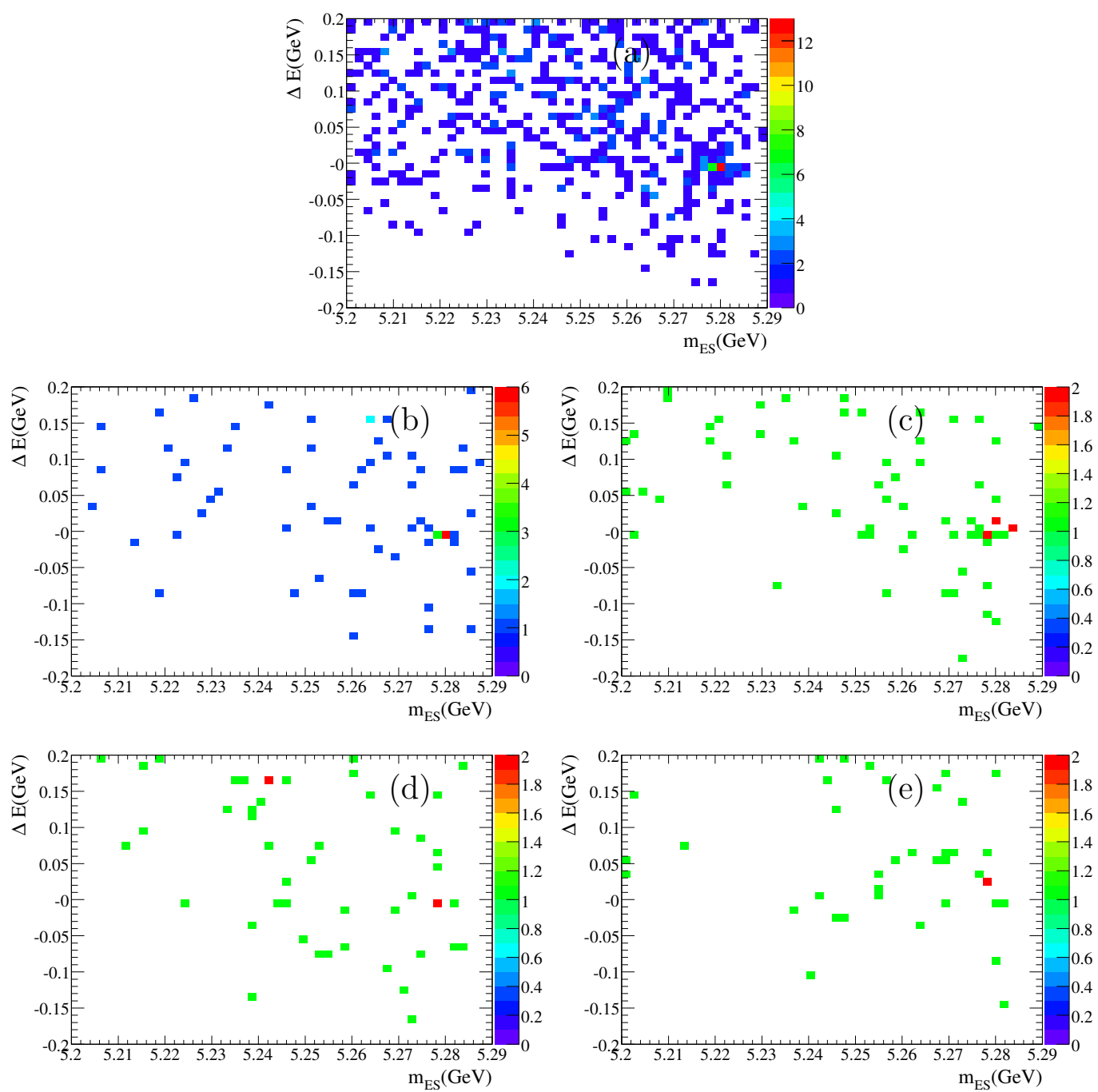

Figure 6.19: $\Delta E$ vs. $m_{\mathrm{ES}}$ distribution of $B^{-} \rightarrow \Lambda_{c}^{+} \bar{\Lambda}_{c}^{-} K^{-}$from on-peak dataset: (a) $\Lambda_{c}^{+} \rightarrow p^{+} K^{-} \pi^{+}, \bar{\Lambda}_{c}^{-} \rightarrow p^{-} K^{+} \pi^{-}$, (b) $\Lambda_{c}^{+} \rightarrow p^{+} K^{-} \pi^{+}, \bar{\Lambda}_{c}^{-} \rightarrow p^{-} K_{S}^{0}$, (c) $\Lambda_{c}^{+} \rightarrow p^{+} K_{S}^{0}$, $\bar{\Lambda}_{c}^{-} \rightarrow p^{-} K^{+} \pi^{-}(\mathrm{d}) \Lambda_{c}^{+} \rightarrow p^{+} K^{-} \pi^{+}, \bar{\Lambda}_{c}^{-} \rightarrow \bar{\Lambda}^{0} \pi^{-}$(e) $\Lambda_{c}^{+} \rightarrow \Lambda^{0} \pi^{+}, \bar{\Lambda}_{c}^{-} \rightarrow p^{-} K^{+} \pi^{-}$. 


\section{CHAPTER 7 \\ $B$ MESON SIGNAL EXTRACTION}

\subsection{Efficiency study}

The detection efficiency $\epsilon$ for each $B$ decay mode is determined from the appropriate signal MC sample, and is computed as the ratio between the number (n) of reconstructed MC candidates passing all the selection cuts (identical to data) and the number $(\mathrm{N})$ of generated signal $B$ events.

$$
\epsilon=\frac{n}{N}
$$

The signal MC datasets listed in the Table 5.3 are used..

\subsubsection{What is a signal event in the MC?}

An important question is how to define a reconstructed signal event. By examining the $m_{\mathrm{ES}}$ distribution of the $B$ candidates from the signal Monte Carlo, two kinds of signal candidates are observed to peak in the signal region: one which satisfies truth-matching ${ }^{1}$ (cat1) and the other where the truth-matching fails (cat2), but the MC event satisfies all the analysis criteria. We allow for final State radiation (FSR) and accept decays with FSR in the truth-matching process (see Section 7.1.2).

We consider the decay mode $B^{-} \rightarrow \Lambda_{c}^{+} \bar{\Lambda}_{c}^{-} K^{-}\left(\Lambda_{c}^{+} \rightarrow p^{+} K^{-} \pi^{+}\right.$for $\Lambda_{c}^{+}$and

\footnotetext{
${ }^{1} \mathrm{~A}$ reconstructed track can have pointers to $\mathrm{MC}$ truth hits (Ghits) associated with it, each with a pointer to the mc truth track (Gtrack) which caused the hit. For each reconstructed track one can determine all the MC-truth tracks which caused the hits on the reconstructed track and select the MC truth-track causing the most hits. This process is called truth-matching
} 
$\left.\bar{\Lambda}_{c}^{-}\right)$as an example. We plot the reconstructed $m_{\mathrm{ES}}$ distribution for all candidates (not using truth-matching information) and perform an unbinned extended maximum likelihood fit. The fitted function is the sum of a single Gaussian (for cat1+cat2), and an Argus function (for background). The fitted signal yield is $7689 \pm 91$. Using truth information to fit the truth-matched (cat1) and non-truth-matched (cat2) yields separately, we find their yield to be $7523 \pm 87$ and $210 \pm 27$, respectively (note that the errors are highly correlated). In other words, the fitted signal yield is about $2 \%$ bigger than the truth-matched signal yield, so we can not simply use the truth-matched yield to compute the efficiency because no truth-matching can be applied to real data.

The key issue is whether or not the candidates in the second category should be considered as part of the signal. The candidates in the second part might have originated from the self-crossfeed ${ }^{2}$ or from inefficiency of the truth-matching procedure. Since the best candidate is already selected for each of these events, and there must be one signal candidate in events with a self-crossfeed candidate, we accept the candidates in category two as part of the signal. We take the fitted yield of the Gaussian as the reconstructed yield $n$ for calculating the efficiency in equation 7.1. Table 7.1 lists the efficiencies of individual channels.

\footnotetext{
${ }^{2}$ In a signal event, a candidate is reconstructed, but some of the final state particles are not correctly assigned in the decay chain. In this case, the candidate is referred to a self-crossfeed candidate.
} 
Table 7.1: Overall efficiency from fitting signal MC

\begin{tabular}{lcc}
\hline \hline Process & $\epsilon_{1}(\%)$ & $\epsilon_{2}(\%)$ \\
\hline$B^{-} \rightarrow \Lambda_{c}^{+} \bar{\Lambda}_{c}^{-} K^{-}$ & & \\
$\Lambda_{c}^{+} \rightarrow p^{+} K^{-} \pi^{+}, \bar{\Lambda}_{c}^{-} \rightarrow p^{-} K^{+} \pi^{-}$ & $6.64 \pm 0.08$ & $6.56 \pm 0.08$ \\
$\Lambda_{c}^{+} \rightarrow p^{+} K^{-} \pi^{+}, \bar{\Lambda}_{c}^{-} \rightarrow p^{-} K_{S}^{0}$ & $8.18 \pm 0.09$ & $8.19 \pm 0.09$ \\
$\Lambda_{c}^{+} \rightarrow p^{+} K_{S}^{0}, \bar{\Lambda}_{c}^{-} \rightarrow p^{-} K^{+} \pi^{-}$ & $8.17 \pm 0.09$ & $8.19 \pm 0.09$ \\
$\Lambda_{c}^{+} \rightarrow p^{+} K^{-} \pi^{+}, \bar{\Lambda}_{c}^{-} \rightarrow \bar{\Lambda}^{0} \pi^{-}$ & $5.91 \pm 0.07$ & $5.90 \pm 0.07$ \\
$\Lambda_{c}^{+} \rightarrow \Lambda^{0} \pi^{+}, \bar{\Lambda}_{c}^{-} \rightarrow p^{-} K^{+} \pi^{-}$ & $6.02 \pm 0.07$ & $5.99 \pm 0.07$ \\
\hline $\bar{B}^{0} \rightarrow \Lambda_{c}^{+} \bar{\Lambda}_{c}^{-} K_{s}^{0}$ & $4.25 \pm 0.06$ & $4.24 \pm 0.06$ \\
\hline$B^{-} \rightarrow \Xi_{c}^{0} \bar{\Lambda}_{c}^{-}$ & & \\
$\Xi_{c}^{0} \rightarrow \Xi^{-} \pi^{+}, \bar{\Lambda}_{c}^{-} \rightarrow p^{-} K^{+} \pi^{-}$ & $4.32 \pm 0.10$ & $4.39 \pm 0.10$ \\
$\Xi_{c}^{0} \rightarrow \Lambda^{0} K^{-} \pi^{+}, \bar{\Lambda}_{c}^{-} \rightarrow p^{-} K^{+} \pi^{-}$ & $4.48 \pm 0.10$ & $4.54 \pm 0.10$ \\
\hline $\bar{B}^{0} \rightarrow \Xi_{c}^{+} \bar{\Lambda}_{c}^{-}$ & $2.60 \pm 0.06$ & $2.61 \pm 0.06$ \\
\hline
\end{tabular}

7.1.2 Effect of final state radiation (FSR) on efficiency

Final State Radiation is a QED process, and the simulation of this process is modeled by the software simulation package PHOTOS [50] in BABAR. The Final State Radiation can take place at several stages of a decay.

- final state radiation photon $(\gamma)$ from B decay;

- final state radiation photon $(\gamma)$ from the first charmed baryon decay;

- final state radiation photon $(\gamma)$ from the second charmed baryon decay;

- final state radiation photon $(\gamma)$ from the intermediate hyperons.

Most of the FSR events contain one photon, only a few contain two. Details are listed in Table ??. Again, the $B^{-} \rightarrow \Lambda_{c}^{+} \bar{\Lambda}_{c}^{-} K^{-}$decay is used as an example. At the 
Table 7.2: Number of photons in the FSR events

\begin{tabular}{lccc}
\hline \hline Process & 1 photon & 2 photons & 3 photons \\
\hline$B^{-} \rightarrow \Lambda_{c}^{+} \bar{\Lambda}_{c}^{-} K^{-}$ & & & \\
$\Lambda_{c}^{+} \rightarrow p^{+} K^{-} \pi^{+}, \bar{\Lambda}_{c}^{-} \rightarrow p^{-} K^{+} \pi^{-}$ & 6211 & 179 & 4 \\
$\Lambda_{c}^{+} \rightarrow p^{+} K^{-} \pi^{+}, \bar{\Lambda}_{c}^{-} \rightarrow p^{-} K_{S}^{0}$ & 5828 & 162 & 2 \\
$\Lambda_{c}^{+} \rightarrow p^{+} K_{S}^{0}, \bar{\Lambda}_{c}^{-} \rightarrow p^{-} K^{+} \pi^{-}$ & 5842 & 165 & 2 \\
$\Lambda_{c}^{+} \rightarrow p^{+} K^{-} \pi^{+}, \bar{\Lambda}_{c}^{-} \rightarrow \bar{\Lambda}^{0} \pi^{-}$ & 7606 & 264 & 4 \\
$\Lambda_{c}^{+} \rightarrow \Lambda^{0} \pi^{+}, \bar{\Lambda}_{c}^{-} \rightarrow p^{-} K^{+} \pi^{-}$ & 7585 & 278 & 6 \\
\hline $\bar{B}^{0} \rightarrow \Lambda_{c}^{+} \bar{\Lambda}_{c}^{-} K_{s}^{0}$ & 7775 & 242 & 9 \\
\hline$B^{-} \rightarrow \Xi_{c}^{0} \bar{\Lambda}_{c}^{-}$ & & & \\
$\Xi_{c}^{0} \rightarrow \Xi^{-} \pi^{+}, \bar{\Lambda}_{c}^{-} \rightarrow p^{-} K^{+} \pi^{-}$ & 2205 & 76 & 2 \\
$\Xi_{c}^{0} \rightarrow \Lambda^{0} K^{-} \pi^{+}, \bar{\Lambda}_{c}^{-} \rightarrow p^{-} K^{+} \pi^{-}$ & 222 & 2 & 1 \\
\hline $\bar{B}^{0} \rightarrow \Xi_{c}^{+} \bar{\Lambda}_{c}^{-}$ & 647 & 14 & 2 \\
\hline
\end{tabular}

generator level, after removing all events with final state radiation, 110606 events out of 117000 events remain, ie., about $5.46 \%$ of signal events have some FSR photons. Among these events, 373 events contain photons from B decays and 6085 from $\Lambda_{c}^{+}$ decays. Although, these photons are not included in the reconstruction, we still accept these events with FSR as signal events. The energy of the FSR photons are very low, as shown in Figure 7.1. Because the FSR photons are not included in the $B$ candidate reconstruction, the mean of the $\Delta E$ distribution gets shifted below 0 in the events with FSR, as shown in Figure 7.2.

The ratio of the number of reconstructed $B$ candidates with FSR to the total reconstructed candidates in the signal MC is about $3 \%$, and the relative efficiency difference of including and not including events with FSR is also about 3\%: not 

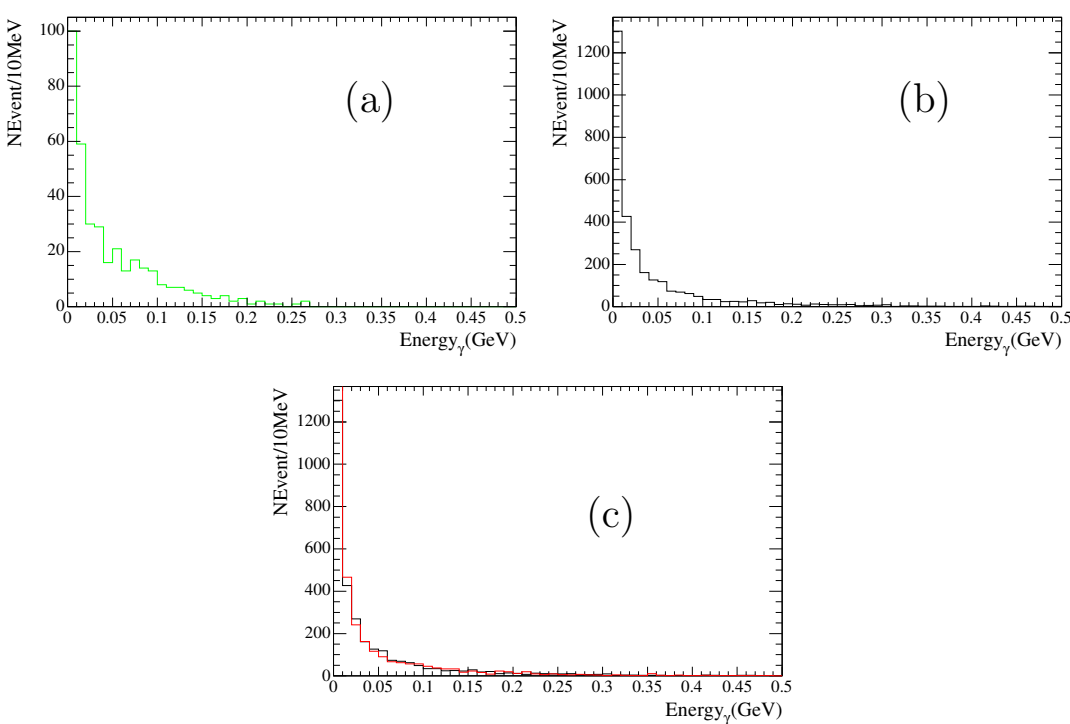

Figure 7.1: The energy of FSR photon in the signal MC for $B^{-} \rightarrow \Lambda_{c}^{+} \bar{\Lambda}_{c}^{-} K^{-}, \Lambda_{c}^{+} \rightarrow$ $p^{+} K^{-} \pi^{+}$. (a)B daughter, (b)the daughter gamma of first $\Lambda_{c}^{+}$, (c) the daughter gamma of second daughter.

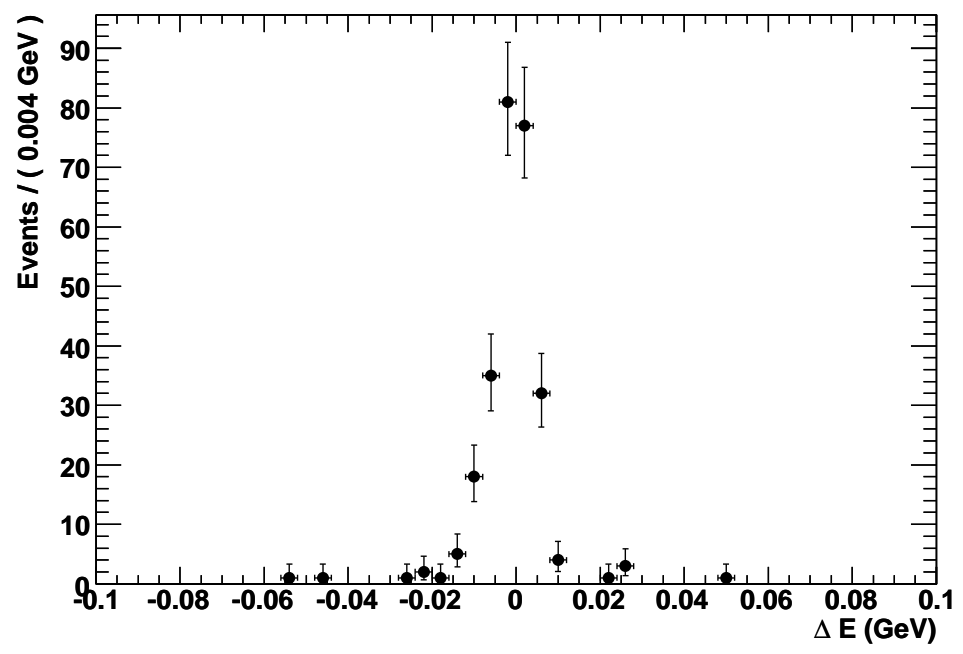

Figure 7.2: The $\Delta E$ distribution of the $B$ candidates $\left(B^{-} \rightarrow \Lambda_{c}^{+} \bar{\Lambda}_{c}^{-} K^{-}, \Lambda_{c}^{+} \rightarrow\right.$ $\left.p^{+} K^{-} \pi^{+}\right)$with FSR photons in the signal MC after all selection cuts, except $\Delta E$, including the best candidate selection. 
a significant difference. Therefore, the small negative shift in the $\Delta E$ distribution caused by the FSR events has very small effect on the $\Delta E$ cut; hence, no correction is applied on the efficiency.

\subsubsection{Tracking efficiency correction}

The tracking efficiency in the MC is observed to be a little higher than that in real data, so a correction is applied to the tracking efficiency according to the recipe proposed by the Track Efficiency Task Force [51]. For all charged tracks $(p, K, \pi)$ derived from the ChargedTracks list, a flat correction of $0.25 \%$ per track is assigned. For the seven decay modes with seven charged tracks in each event, the correction is $(1-0.0025)^{7}$, or 0.9826 per event. The $\bar{B}^{0} \rightarrow \Lambda_{c}^{+} \bar{\Lambda}_{c}^{-} K_{s}^{0}$ and $\bar{B}^{0} \rightarrow \Xi_{c}^{+} \bar{\Lambda}_{c}^{-}$modes contain eight charged tracks in each event, resulting in a correction factor of $(1-$ $0.0025)^{8}$, or 0.9802. Systematic uncertainties are discussed in Section 7.4.

\subsubsection{Effect of intermediate resonance on efficiency}

In the signal Monte Carlo, the three-body $\Lambda_{c}^{+} \rightarrow p^{+} K^{-} \pi^{+}$decays are generated according to phase space, without considering any intermediate resonances (e.g. $K^{*}, \Delta^{++}$), which could very well be present in real data resulting in a non-uniform Dalitz plot distribution. The ratios of decays widths through the intermediate resonant states have been measured previously and are non-negligible:

$$
\Gamma\left(\left(p K^{-} \pi^{+}\right)_{n o n-r e s}\right) / \Gamma_{\text {total }}=0.55 \pm 0.06, \Gamma\left(\Delta^{++} K^{-}\right) / \Gamma_{\text {total }}=0.17 \pm 0.04, \text { and }
$$

$\Gamma\left(p K^{*}\right) / \Gamma_{\text {total }}=0.31 \pm 0.04$

However, modeling these decays with proper interference effects is a very sub- 
stantial effort, especially given the limited scope in statistics. We take a pragmatic approach to study the effect on the efficiency, generating several exclusive MC samples including resonant states as listed in Table 7.3. In each sample, only one combination of $\Lambda_{c}^{+}$and $\bar{\Lambda}_{c}^{-}$final state is included. The results of the efficiency calculation with these intermediate states are summarized in Table 7.3, where all selection cuts are applied, including the best candidate selection. The efficiencies are statistically consistent, so we do not assign any correction or systematic uncertainty.

Table 7.3: Summary of efficiencies for various assumptions about intermediate resonances in $\Lambda_{c}^{+} \rightarrow p^{+} K^{-} \pi^{+}$decays, measured from 4 signal MC samples

\begin{tabular}{cc}
\hline$B^{-} \rightarrow \Lambda_{c}^{+} \bar{\Lambda}_{c}^{-} K^{-}$ & Efficiency $(\%)$ \\
\hline$\Lambda_{c}^{+} \rightarrow p^{+} K^{-} \pi^{+}, \bar{\Lambda}_{c}^{-} \rightarrow p^{-} K^{+} \pi^{-}$ & $6.65 \pm 0.18$ \\
$\Lambda_{c}^{+} \rightarrow p^{+} K^{-} \pi^{+}, \bar{\Lambda}_{c}^{-} \rightarrow \bar{\Delta}^{--} K^{+}$ & $6.62 \pm 0.18$ \\
$\Lambda_{c}^{+} \rightarrow p^{+} K^{-} \pi^{+}, \bar{\Lambda}_{c}^{-} \rightarrow p^{-} \bar{K}^{* 0}$ & $6.5 \pm 0.17$ \\
$\Lambda_{c}^{+} \rightarrow \Delta^{++} K^{-}, \bar{\Lambda}_{c}^{-} \rightarrow p^{-} \bar{K}^{* 0}$ & $6.74 \pm 0.18$ \\
\hline
\end{tabular}

7.1.5 The crosscheck of efficiencies of three-body $B$ decays

The signal MC of the three-body $B$ decay, $B^{-} \rightarrow \Lambda_{c}^{+} \bar{\Lambda}_{c}^{-} K^{-}$is also generated assuming no intermediate resonances are present, ie. distributed according to phase space. There is currently no experimental evidence for the presence of any intermediate resonance in this decay (discussed later in Section 8.3). One possible intermediate resonance in the $B$ decay is $\Xi_{c}^{*}(2980) \rightarrow \Lambda_{c}^{+} K^{-}$. Figure 7.3 shows the 


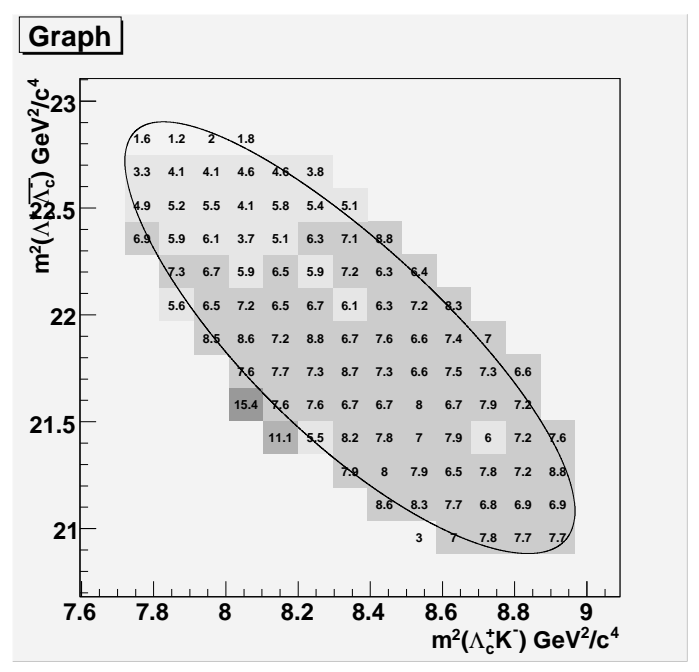

Figure 7.3: 2-D efficiency for the decay mode $B^{-} \rightarrow \Lambda_{c}^{+} \bar{\Lambda}_{c}^{-} K^{-}$.

efficiency distribution in the Dalitz plane $m^{2}\left(\Lambda_{c}^{+} K^{-}\right)$vs. $m^{2}\left(\Lambda_{c}^{+} \bar{\Lambda}_{c}^{-}\right)$for this decay mode with $\Lambda_{c}^{+} \rightarrow p^{+} K^{-} \pi^{+}$. The local efficiency varies relatively smoothly, except at the top-left corner where the $K^{-}$has low momentum in the $B$ rest frame, and the efficiency drops noticeably. But the same region in data has almost no statistics (even allowing for the drop in efficiency), the impact on the efficiency-corrected yield is negligible. Therefore, the global signal efficiency is used.

\subsection{The maximum likelihood fit and and fit validation}

\subsubsection{Unbinned maximum likelihood fit}

To extract the signal yields from data, an unbinned extended maximum likelihood fit is performed to distinguish the signal distribution from the background. The fit is done by the MINUIT fit program package [53] interfaced with ROOT [54] by 
using software built upon the RooFit tool kit [55]. The likelihood function is defined as

$$
\mathcal{L}\left(\vec{x}, \vec{p}, n_{1}, \ldots, n_{m}\right)=\frac{\exp \left(-\sum_{j=1}^{m} n_{j}\right)}{N !} \prod_{i=1}^{N}\left(\sum_{j=1}^{m} n_{j} \mathcal{P}_{j}(\vec{x}, \vec{p})\right)
$$

where $\vec{x}$ is a set of observable discriminating variables, $\vec{p}$ is a set of shape parameters, and $n_{j}$ is the number of candidates in the $j$ th category. $\mathcal{P}_{j}(\vec{x}, \vec{p})$ is the Probability Density Function (PDF) for the $j$ th category. The modeling of $\mathcal{P}_{j}(\vec{x})$ is explained in the following section. $N$ is the number of events measured, which is assumed to follow a Poisson distribution around the underlying expected value.

By minimizing the negative logarithm of the extended likelihood,

$$
\begin{aligned}
& \frac{\partial \ln \mathcal{L}}{\partial \vec{p}}=0, \\
& \frac{\partial \ln \mathcal{L}}{\partial n_{j}}=0, \quad j=1, \ldots, m .
\end{aligned}
$$

the maximum likelihood (ML) estimator can be obtained.

\subsubsection{The modeling of the PDF}

The variable $m_{\mathrm{ES}}$ is used as the discriminating variable to model the signal and separate it from the background; therefore a one dimensional fit on the variable $m_{\mathrm{ES}}$ is performed. Events from the data samples are assumed to consist of two components: signal and combinatorial backgrounds.

Signal shape: The signal PDF is determined from the signal MC. We describe them as a single Gaussian for each decay as shown below. This describes all peaking, signal-like events including decays with the FSR and the self-cross-feed (see 
Section 7.1.1).

$$
\mathcal{P}_{\text {sig }}\left(m_{\mathrm{ES}} ; \mu_{m_{E S}}, \sigma\right)=e^{\frac{-\left(m_{E S}-\mu_{m_{E S}}\right)^{2}}{2 \sigma^{2}}}
$$

The parameters $\mu_{\mathrm{m}_{E S}}$ and $\sigma$ are either fixed to the those obtained from fits to the signal MC, or float the fit to the data for different decay modes.

Background shape: We investigate and validate the shape of the background $\mathrm{PDF}$ with the $B \bar{B}$ generic $\mathrm{MC}$ and the $\Delta E$ sideband data. It contains only combinatoric background arising from random combinations of tracks. It is parameterized by the Argus function, shown below:

$$
\mathcal{P}_{b k g}\left(m_{\mathrm{ES}} ; c, m_{0}\right)=m_{\mathrm{ES}} \cdot \sqrt{1-\left(\frac{m_{\mathrm{ES}}}{m_{0}}\right)^{2}} \cdot e^{c\left(1-\left(\frac{\mathrm{m}_{E S}}{m_{0}}\right)^{2}\right)}
$$

where $m_{0}$, a threshold parameter ${ }^{3}$ of ARGUS function, is fixed to the beam energy in the $\mathrm{CM}$ frame, $5.290 \mathrm{GeV} / c^{2}$, and $c$ is a dimensionless shape parameter determined by the fit.

\subsubsection{Fit strategy and result}

All parameters of the background are left free in the fit except the endpoint; the initial value of the shape parameter $c$ is taken from the generic MC and $\Delta E$ sideband in the data, both of which yield similar values. A simultaneous fit is performed for all five final states used in $B^{-} \rightarrow \Lambda_{c}^{+} \bar{\Lambda}_{c}^{-} K^{-}$. For the charged three-body modes, the $\sigma$ of the Gaussian is fixed to that obtained from the signal MC with the mean left free in the $m_{\mathrm{ES}}$ fit. However, because of low statistics for the two-body decay modes and

\footnotetext{
${ }^{3}$ In reality, the endpoint in the $m_{\mathrm{ES}}$ distribution is not a single point, because the beam energy shifts between runs. But the change is small, so we can accept it as a point in our analysis
} 

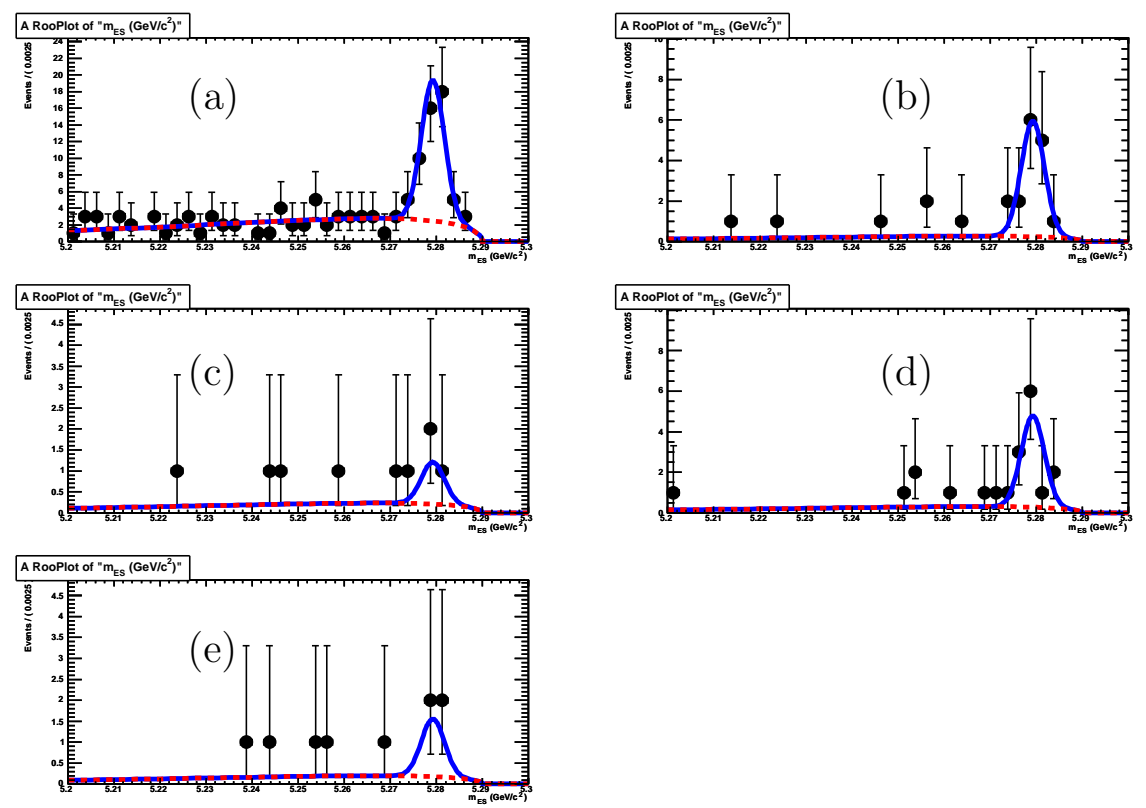

Figure 7.4: $m_{\mathrm{ES}}$ fit of $B^{-} \rightarrow \Lambda_{c}^{+} \bar{\Lambda}_{c}^{-} K^{-}$to the Run 1-4 on-peak data. (a) $\Lambda_{c}^{+} \rightarrow$ $p^{+} K^{-} \pi^{+}, \bar{\Lambda}_{c}^{-} \rightarrow p^{-} K^{+} \pi^{-} ;(\mathrm{b}) \Lambda_{c}^{+} \rightarrow p^{+} K^{-} \pi^{+}, \bar{\Lambda}_{c}^{-} \rightarrow p^{-} K_{S}^{0}$; (c) $\Lambda_{c}^{+} \rightarrow p^{+} K^{-} \pi^{+}$, $\bar{\Lambda}_{c}^{-} \rightarrow \bar{\Lambda}^{0} \pi^{-} ;$(d) $\Lambda_{c}^{+} \rightarrow p^{+} K_{S}^{0}, \bar{\Lambda}_{c}^{-} \rightarrow p^{-} K^{+} \pi^{-} ;(\mathrm{e}) \Lambda_{c}^{+} \rightarrow \Lambda^{0} \pi^{+}, \bar{\Lambda}_{c}^{-} \rightarrow p^{-} K^{+} \pi^{-}$

the neutral three-body decay mode, the $\sigma$ and the mean of the Gaussian, are both fixed to the values obtained from the signal MC. In both two-body and three-body decays, the signal yield as well as the shape parameter of the Argus function and the background yield are floated separately for each individual sub-mode.

Figure 7.4 and 7.5 show the fit result for $B^{-} \rightarrow \Lambda_{c}^{+} \bar{\Lambda}_{c}^{-} K^{-}$in the data. The yield of the signal in each decay mode is listed in the Table 7.4.

Figure 7.6 shows the fit result for $B^{-} \rightarrow \Xi_{c}^{0} \bar{\Lambda}_{c}^{-}$with (a) $\Xi_{c}^{0} \rightarrow \Xi^{-} \pi^{+}$, (b) $\Xi_{c}^{0} \rightarrow \Lambda^{0} K^{-} \pi^{+}$

Figure 7.7 shows (a) the fit for $\bar{B}^{0} \rightarrow \Xi_{c}^{+} \bar{\Lambda}_{c}^{-}$, (b) shows the fit for $\bar{B}^{0} \rightarrow$ 


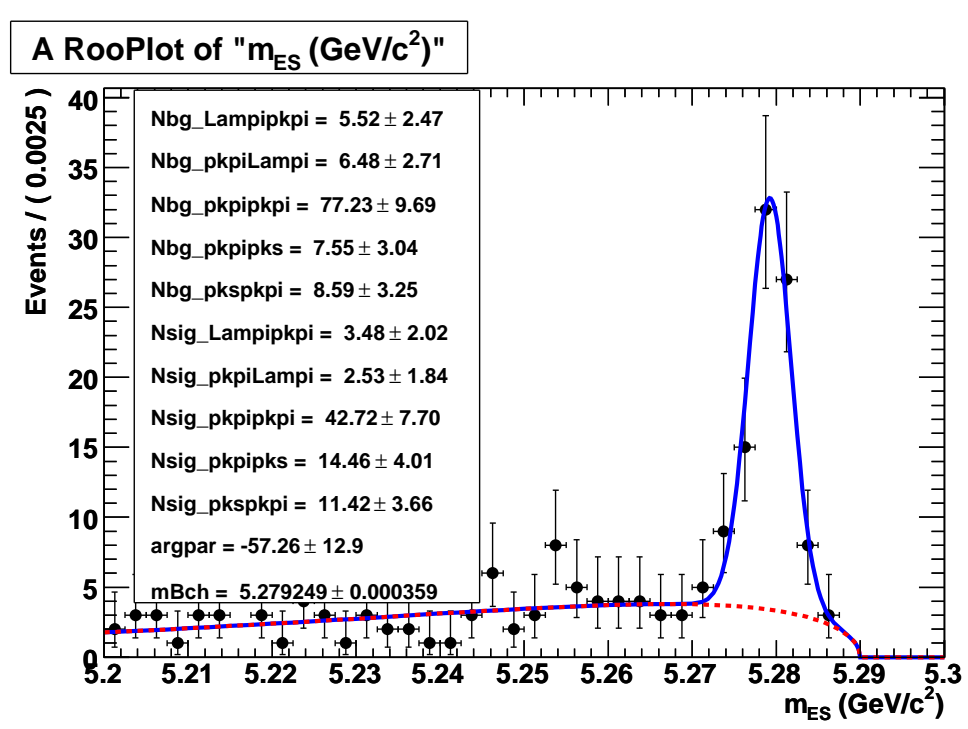

Figure 7.5: $m_{\mathrm{ES}}$ fit of $B^{-} \rightarrow \Lambda_{c}^{+} \bar{\Lambda}_{c}^{-} K^{-}$to the Run 1-4 on-peak data in all five modes.
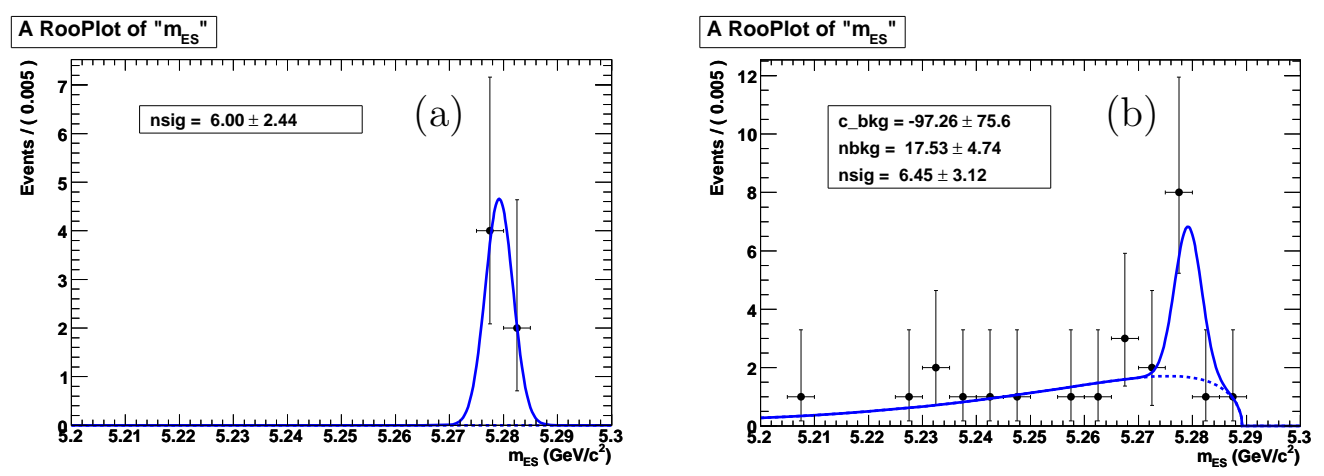

Figure 7.6: $m_{\mathrm{ES}}$ fit of $B^{-} \rightarrow \Xi_{c}^{0} \bar{\Lambda}_{c}^{-}:(\mathrm{a}) \Xi_{c}^{0} \rightarrow \Xi^{-} \pi^{+}$, and (b) $\Xi_{c}^{0} \rightarrow \Lambda^{0} K^{-} \pi^{+}$(right) 
$\Lambda_{c}^{+} \bar{\Lambda}_{c}^{-} K_{s}^{0}$. The signal yield for each decay mode is listed in Table 7.4.
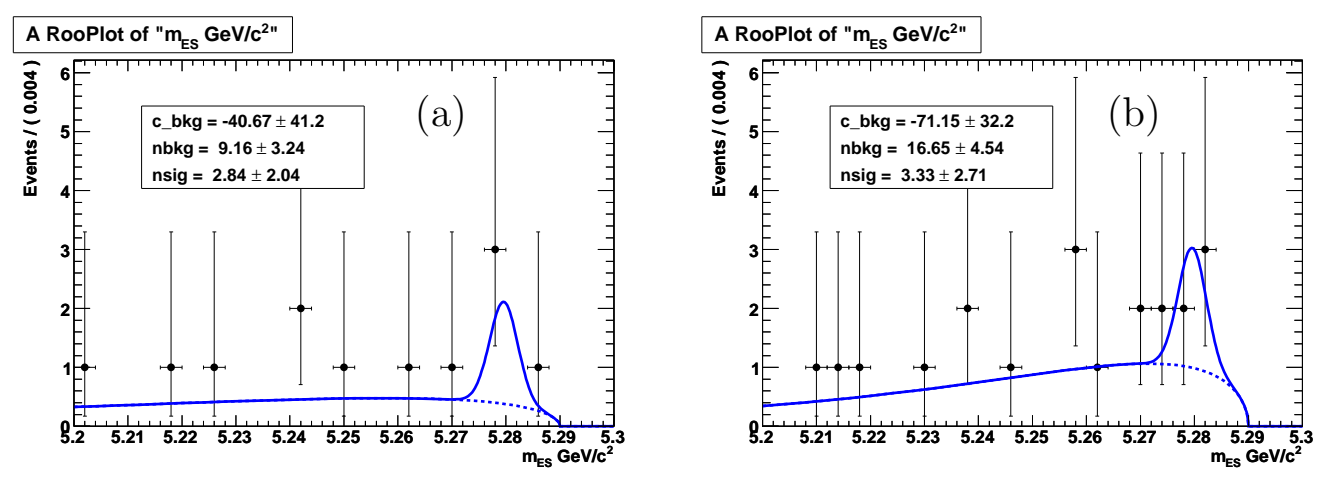

Figure 7.7: $m_{\mathrm{ES}}$ fit of (a) $\bar{B}^{0} \rightarrow \Xi_{c}^{+} \bar{\Lambda}_{c}^{-}$, (b) $\bar{B}^{0} \rightarrow \Lambda_{c}^{+} \bar{\Lambda}_{c}^{-} K_{s}^{0}$

Table 7.4: Fitted yields from the Run 1-4 on-peak data and likelihood difference $\Delta \log \mathcal{L}$ between the fits with signal plus background and with background only.

\begin{tabular}{lcc}
\hline \hline Decay Mode & Yield & Significance \\
\hline$B^{-} \rightarrow \Lambda_{c}^{+} \bar{\Lambda}_{c}^{-} K^{-}$ & & 6 \\
$\Lambda_{c}^{+} \rightarrow p^{+} K^{-} \pi^{+}, \bar{\Lambda}_{c}^{-} \rightarrow p^{-} K^{+} \pi^{-}$ & $42.7 \pm 7.7$ & \\
$\Lambda_{c}^{+} \rightarrow p^{+} K^{-} \pi^{+}, \bar{\Lambda}_{c}^{-} \rightarrow p^{-} K_{S}^{0}$ & $14.5 \pm 4.0$ & \\
$\Lambda_{c}^{+} \rightarrow p^{+} K_{S}^{0}, \bar{\Lambda}_{c}^{-} \rightarrow p^{-} K^{+} \pi^{-}$ & $11.4 \pm 3.7$ & \\
$\Lambda_{c}^{+} \rightarrow p^{+} K^{-} \pi^{+}, \bar{\Lambda}_{c}^{-} \rightarrow \bar{\Lambda}^{0} \pi^{-}$ & $2.5 \pm 1.8$ & \\
$\Lambda_{c}^{+} \rightarrow \Lambda^{0} \pi^{+}, \bar{\Lambda}_{c}^{-} \rightarrow p^{-} K^{+} \pi^{-}$ & $3.5 \pm 2.0$ & 2 \\
\hline $\bar{B}^{0} \rightarrow \Lambda_{c}^{+} \bar{\Lambda}_{c}^{-} K_{s}^{0}$ & $3.3 \pm 2.7$ & 3 \\
\hline$B^{-} \rightarrow \Xi_{c}^{0} \bar{\Lambda}_{c}^{-}$ & & \\
$\Xi_{c}^{0} \rightarrow \Xi^{-} \pi^{+}, \bar{\Lambda}_{c}^{-} \rightarrow p^{-} K^{+} \pi^{-}$ & $6.0 \pm 2.4$ & 2 \\
$\Xi_{c}^{0} \rightarrow \Lambda^{0} K^{-} \pi^{+}, \bar{\Lambda}_{c}^{-} \rightarrow p^{-} K^{+} \pi^{-}$ & $7.5 \pm 3.1$ & \\
\hline $\bar{B}^{0} \rightarrow \Xi_{c}^{+} \bar{\Lambda}_{c}^{-}$ & $2.8 \pm 2.0$ & \\
\hline
\end{tabular}




\subsection{Background Study}

In order to extract the correct $B$ signal yield, the background underlaying the $B$ signal in the $m_{\mathrm{ES}}$ distribution needs to be clearly understood and characterized. In general, the background is composed of two parts, peaking background and combinatorial background. The combinatorial background comes from random combinations of tracks from both $B \bar{B}$ and continuum in general. It is distributed smoothly in the $m_{\mathrm{ES}}$ and $\Delta E$ plane. The $B$-related background consists of $B$ meson decays into a different final state than the signal mode but mimics a signal mode, passing all the selection cuts and populating the signal region. This type of background often peaks around the $B$ mass in the $m_{\mathrm{ES}}-\Delta E$ plane, which is why it is called peaking background. As described in Section 7.2, we parameterize the background PDF by an Argus function, based on studies of generic MC and $\Delta E$ sideband data. In this section we consider the possible sources of peaking background in more detail. The dominant sources are from various $b \rightarrow c \bar{u} d$ processes.

\subsubsection{Background study of the $B \rightarrow \Xi_{c} \bar{\Lambda}_{c}^{-}$decay modes}

Since the pattern of baryonic $B$ decays is still being understood experimentally, it is not modeled well in the generic MC. Many different MC samples are generated to understand the possible sources of the background. The results from such studies are described below. 


\subsubsection{Background from $B \rightarrow \Xi_{c} \bar{\Lambda}_{c}+n \pi($ or $\gamma)$ decays}

As we observed in the on-peak data in Figure 6.18(a), the 2-D scatter plot of $m_{\mathrm{ES}}$ vs. $\Delta E$ shows an excess of events in the region of $5.27 \mathrm{GeV}<m_{\mathrm{ES}}<5.29 \mathrm{GeV}$ and $\Delta E<-0.5 \mathrm{GeV}$. We generate and analyze several signal MC samples with similar topologies to the desired signal decays, where one or more extra soft pions or $\gamma$ from the $B, \Xi_{c}^{*\left({ }^{\prime}\right)}$ or $\Sigma_{c}$ are missed in the $B$ reconstruction. The decay modes investigated are:

- $\bar{B}^{0} \rightarrow \Xi_{c}^{+}{\overline{\Sigma_{c}}}^{-}\left(\pi^{0}\right)$, and $B^{-} \rightarrow \Xi_{c}^{0}{\overline{\Sigma_{c}}}^{-}\left(\pi^{0}\right)$, where $\Sigma_{c}^{+} \rightarrow \Lambda_{c}^{+} \pi^{0}$. ( $\left.\pi^{0}\right)$ means that three-body decays are also included.

- $B^{-} \rightarrow \Xi_{c}^{* 0}{\overline{\Sigma_{c}}}^{-}\left(\pi^{0}\right)$, where $\Xi_{c}^{* 0} \rightarrow \Xi_{c}^{0} \pi^{+}$

- $\bar{B}^{0} \rightarrow \Xi_{c}^{0}{\overline{\Sigma_{c}}}^{0}$, where $\Sigma_{c}^{0} \rightarrow \Lambda_{c}^{+} \pi^{-}$

- $B^{-} \rightarrow \Xi_{c}^{+}{\overline{\Sigma_{c}}}^{--}$, where $\Sigma_{c}^{++} \rightarrow \Lambda_{c}^{+} \pi^{+}$

- $B^{-} \rightarrow \Xi_{c}^{0} \bar{\Lambda}_{c}^{-} \pi^{0}, \bar{B}^{0} \rightarrow \Xi_{c}^{0} \bar{\Lambda}_{c}^{-} \pi^{+}$, and $B^{-} \rightarrow \Xi_{c}^{+} \bar{\Lambda}_{c}^{-} \pi^{-}$

- $B^{0} \rightarrow \Xi_{c}^{*+} \bar{\Lambda}_{c}^{-}, B^{-} \rightarrow \Xi_{c}^{* 0} \bar{\Lambda}_{c}^{-}$, where $\Xi_{c}^{*} \rightarrow \Xi_{c} \pi$

- $B^{-} \rightarrow \Xi_{c}^{\prime} \bar{\Lambda}_{c}^{-}$, where $\Xi_{c}^{\prime 0} \rightarrow \Xi_{c}^{0} \gamma$

Branching ratio measurements for these decay modes do not exist; theoretical predictions for the two-body decays are about $0.1 \sim 0.5 \times 10^{-3}$ for our signal modes.

The plots in figure 7.8 show the $\Delta E$ vs. $m_{\mathrm{ES}}$ distributions of the mis-reconstructed $B$ from several signal MC samples: (a) $B^{-} \rightarrow \Xi_{c}^{\prime 0} \bar{\Lambda}_{c}^{-}$where the soft $\gamma$ from the $\Xi_{c}^{\prime+}$ 
is not included; (b) $B^{-} \rightarrow \Xi_{c}^{+}{\overline{\Sigma_{c}}}^{--}$where the soft $\pi^{+}$from the $\Sigma_{c}^{++}$is not included; (c) $B^{-} \rightarrow \Xi_{c}^{+} \bar{\Lambda}_{c}^{-} \pi^{-}$where the bachelor $\pi^{+}$from the $B$ is not included; (d) $\bar{B}^{0} \rightarrow \Xi_{c}^{+} \bar{\Lambda}_{c}^{-} \pi^{+} \pi^{-}$where the two bachelor pions are not included. Of these four typical modes, the one in (a) contaminates the signal most. With a cut on $\Delta E$ of $|\Delta E|<22 \mathrm{MeV}$ which corresponds approximately to a $3 \sigma$ window, these backgrounds can be essentially removed with little loss of signal efficiency. The background shown in Figure 7.8 (b),(c) and (d) peak even further away from the signal in $\Delta E$ and are therefore removed by this cut as well.
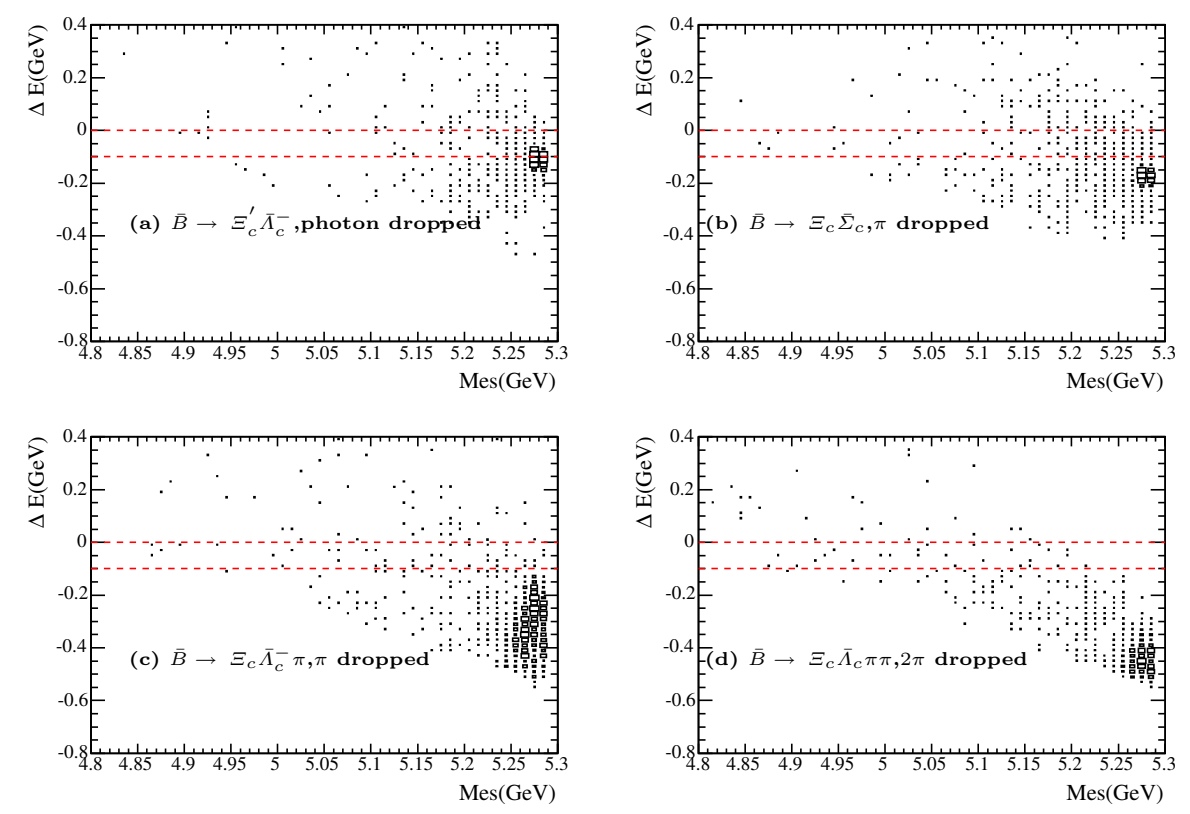

Figure 7.8: The distribution of $m_{\mathrm{ES}}$ vs. $\Delta E$ for the 4 example feed-down modes in which a pion, two pions or a $\gamma$ not included. 
A similar situation can arise for the $B^{-} \rightarrow \Xi_{c}^{0} \bar{\Lambda}_{c}^{-}$modes reconstructed with contamination from $B \rightarrow \Xi_{c}^{0} \bar{\Lambda}_{c}^{-}(m \pi)(\gamma)$ (resonant states are implied). Figure 7.9 shows the $\Delta E$ distributions from the signal mode $B^{-} \rightarrow \Xi_{c}^{0} \bar{\Lambda}_{c}^{-}$and several of these background modes. Again, these typically peak in the $m_{\mathrm{ES}}>5.27 \mathrm{GeV}$ signal region, but the backgrounds accumulate at $\Delta E<-0.05 \mathrm{GeV}$. As shown, the $\Delta E$ variable separates between the signal modes from these feed-down modes clearly. A cut of $|\Delta E|<22 \mathrm{MeV}$ removes all the potential contamination from these modes.

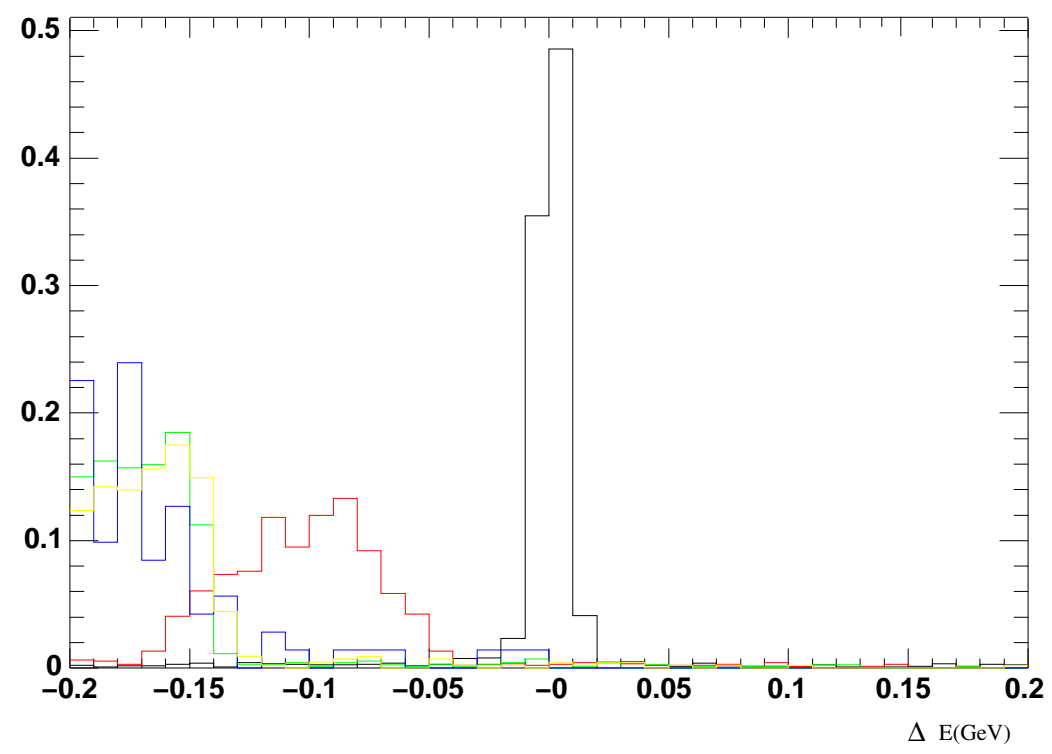

Figure 7.9: $\Delta E$ distribution of $B^{-} \rightarrow \Xi_{c}^{0} \bar{\Lambda}_{c}^{-}$from the $B^{-} \rightarrow \Xi_{c}^{0} \bar{\Lambda}_{c}^{-}$signal MC and cross-feed mode(normalized distribution). The black histogram shows the signal mode $B^{-} \rightarrow \Xi_{c}^{0} \bar{\Lambda}_{c}^{-}$. The colored histograms show contributions from example feeddown modes: $B^{-} \rightarrow \Xi_{c}^{0} \bar{\Lambda}_{c}^{-}$(red), $\bar{B}^{0} \rightarrow \Xi_{c}^{0} \bar{\Lambda}_{c}^{-} \pi^{+}$(green), $B^{-} \rightarrow \Xi_{c}^{0} \bar{\Lambda}_{c}^{-} \pi^{0}$ (blue), $B^{-} \rightarrow \Xi_{c}^{0} \bar{\Sigma}_{c}^{-} \pi^{0}$ (yellow). 


\subsubsection{Background from $b \rightarrow c \bar{u} d$ processes}

We search for additional sources of peaking background with the generic MC samples. These events contain a very small amount of true $B \rightarrow \Xi_{c} \bar{\Xi}_{c} \pi^{0}$ and $B \rightarrow$ $\Lambda_{c}^{+} \bar{\Lambda}_{c}^{-} K$, and no true $B^{-} \rightarrow \Xi_{c}^{0} \bar{\Lambda}_{c}^{-}$or $\bar{B}^{0} \rightarrow \Xi_{c}^{+} \bar{\Lambda}_{c}^{-}$, so peaking structure in these modes indicates a possible source of background. Figures 7.10 show the (a) $m_{\mathrm{ES}}$, (b) $\Delta E$ vs. $m_{\mathrm{ES}}$, and (c) the $\Delta E$ distribution of the $B$ candidates $\left(B^{-} \rightarrow \Xi_{c}^{0} \bar{\Lambda}_{c}^{-}, \Xi_{c}^{0} \rightarrow\right.$ $\left.\Xi^{-} \pi^{+}, \Lambda_{c}^{+} \rightarrow p^{+} K^{-} \pi^{+}\right)$reconstructed from the generic $B^{+} B^{-}$MC sample. The reconstruction and selection procedures used are exactly the same as those for the data, except that the charmed baryon masses are not constrained to the nominal values in the fit. A peak is observed in the signal region $\left(m_{\mathrm{ES}}>5.27 \mathrm{GeV}\right.$, and $\left.|\Delta E|<3 \sigma_{\Delta E}\right)$. A second, large peak is observed in the $\Delta E$ sideband $\left(m_{\mathrm{ES}}>\right.$ $5.27 \mathrm{GeV}$, and $\Delta E<-0.15 \mathrm{GeV})$.

These peaking structures are from the $b \rightarrow c \bar{u} d$ processes. In the fit region of $|\Delta E|<0.03 \mathrm{GeV}$ and $5.2<m_{E S}<5.3 \mathrm{GeV}, 29$ events are left after all selection cuts. Out of these, 16 events are in the $|\Delta E|<0.03 \mathrm{eV}$ and $m_{E S}>5.27 \mathrm{GeV}$ region, which is the signal region. From a detailed study of these 16 events, we find

- 6 events: $B^{-} \rightarrow \Xi_{c}^{0} \bar{\Delta}^{--} K^{+}$

- 3 events: $B^{-} \rightarrow \Xi_{c}^{0} \bar{p} \bar{K}^{* 0}$

- 7 events: Random combinations which do not form a fully reconstructed $B$ meson.

The $m_{\mathrm{ES}}$ distribution is fitted with the model described in the previous section, and a signal yield of $13 \pm 4$, shown in Figure 7.11, is obtained; this is consistent with the 

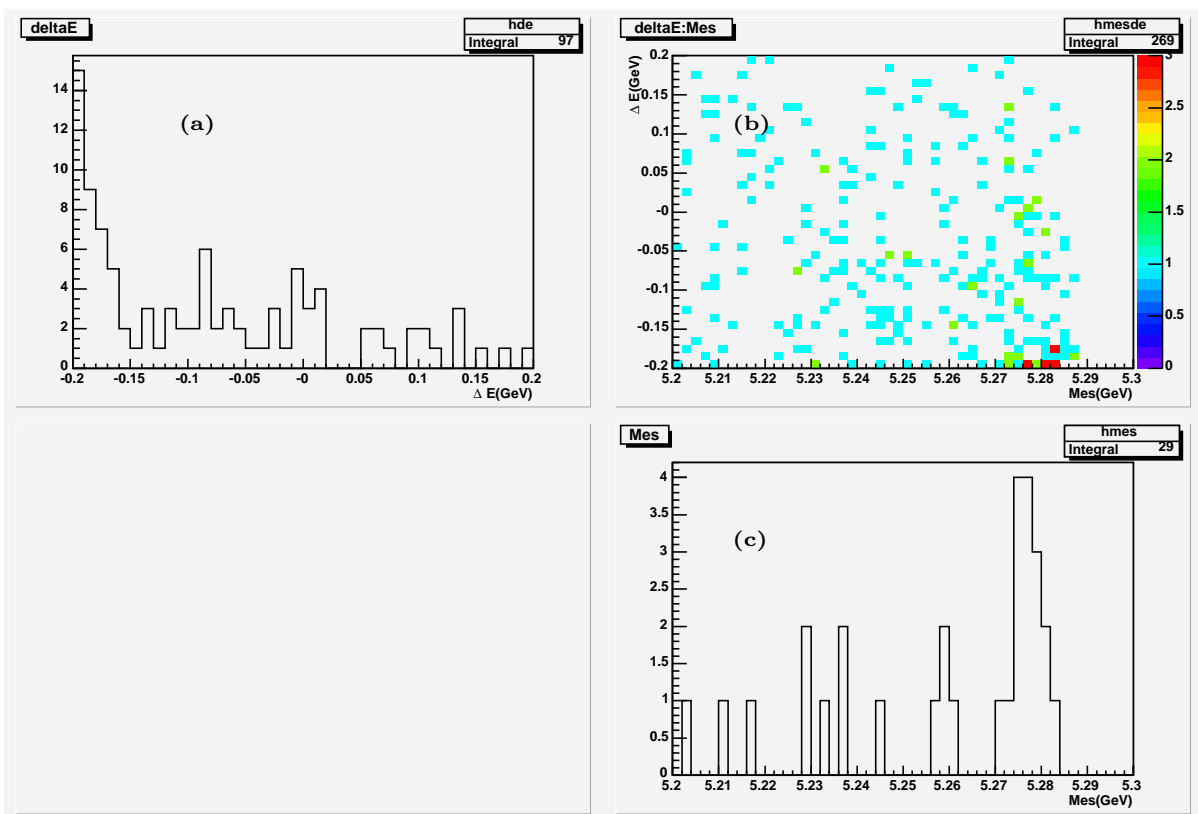

Figure 7.10: The $m_{\mathrm{ES}}$ and $\Delta E$ distributions of $B^{-} \rightarrow \Xi_{c}^{0} \bar{\Lambda}_{c}^{-}$reconstructed from Generic $B^{+} B^{-}$MC. (a) $\Delta E$ distribution with $m_{\mathrm{ES}}>5 / 27 \mathrm{GeV}$, (b) 2 d plot of $\Delta E$ vs. $m_{\mathrm{ES}}(\mathrm{c}) m_{\mathrm{ES}}$ distribution with $|\Delta E|<0.03 \mathrm{GeV}$

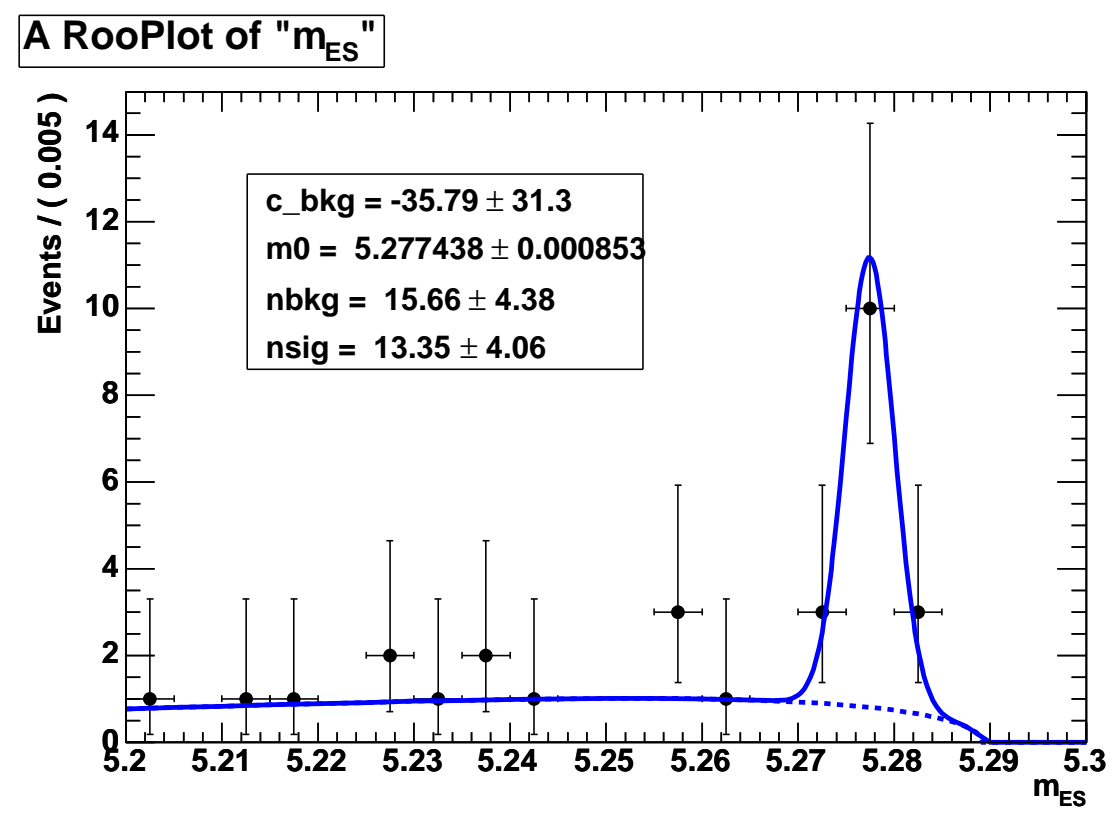

Figure 7.11: $m_{\mathrm{ES}}$ distribution from generic $B^{+} \bar{B}^{-}$. 
nine signal events within the statistical uncertainty. It is not surprising that $B^{-} \rightarrow$ $\Xi_{c}^{0} \bar{p} \bar{K}^{* 0}, B^{-} \rightarrow \Xi_{c}^{0} \bar{\Delta}^{--} K^{+}$, and $B^{-} \rightarrow \Xi_{c}^{0} \bar{p} K^{+} \pi^{-}$can cause peaking background, as these modes have the same particles in the final state as the signal mode. To evaluate the effect of such contaminations in the signal, 11,000 MC events are generated in each of these modes as well as the signal modes. By applying the same selection cuts for both of these samples, the ratio of the detection efficiency of these peaking background modes and the signal mode is estimated to be about $5 \%(0.0019 / 0.043)$. The difference in efficiency is due to the tight charmed baryon mass cut. Even though this ratio is quite small, since we don't know the relative branching fractions of the processes, it cannot simply be ignored.
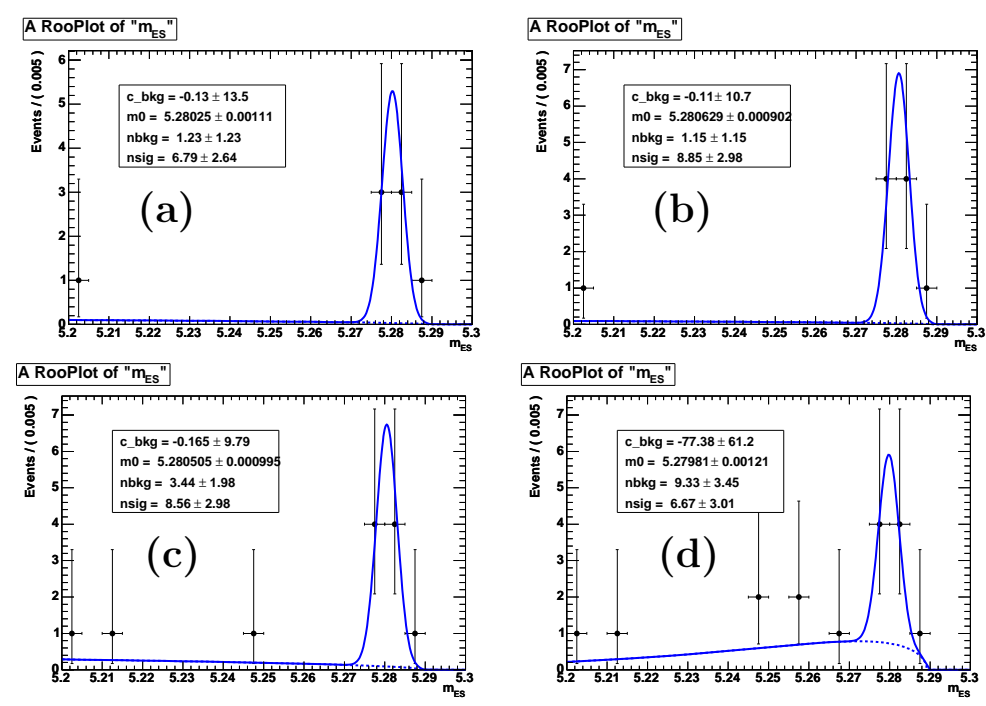

Figure 7.12: $m_{\mathrm{ES}}$ distribution of the $B$ candidate $\left(\Xi_{c}^{0} \bar{\Lambda}_{c}^{-}\right)$with different cut on the invariant mass of $p K \pi$ from the run 1-4 data. (a) $\left|m_{p K \pi}-\bar{m}\right|<2 \sigma$; (b) $\left|m_{p K \pi}-\bar{m}\right|<$ $3 \sigma ;$ (c) $\left|m_{p K \pi}-\bar{m}\right|<6 \sigma ;$ (d) $\left|m_{p K \pi}-\bar{m}\right|<9 \sigma$ 
We can estimate this background with the data, taking advantage of the fact that it does not peak in $m(p K \pi)$. We repeat the analysis with increasingly wide $m(p K \pi)$ windows and find that the signal peak remains constant within errors, even out to $\pm 9 \sigma$, as shown in Figure 7.12. Hence, we conclude that this is not a significant source of peaking background.

\subsubsection{Background from $\bar{B} \rightarrow \Xi_{c} X$ and $B \rightarrow \bar{\Lambda}_{c}^{-} Y$ in the same event}

Yet another possible source of peaking background could arise when one $B$ decay includes a $\Xi_{c}$ and the other B decay includes a $\bar{\Lambda}_{c}^{-}$, and this is misinterpreted as an event in which both the $\Xi_{c}$ and the $\bar{\Lambda}_{c}^{-}$are decay products of the same $B$. This effect is also evaluated with 10,000 MC events generated for this study in the decay modes: $B^{0} \rightarrow \bar{\Lambda}_{c}^{-} p^{+} \pi^{-} \pi^{+}$, and, $\bar{B}^{0} \rightarrow \Xi_{c}^{+} \bar{\Lambda}^{0} \pi^{-}$. (Note that no exclusive $B$ decay to $\Xi_{c}$ is possible without a second charmed baryon in the final state.) Events from this dataset are reconstructed as $\bar{B}^{0} \rightarrow \Xi_{c}^{+} \bar{\Lambda}_{c}^{-}$as before. The mass distributions of $\Xi_{c}^{+}$ and $\Lambda_{c}^{+}$are shown in Fig.s 7.13 (a),(b) and (c), and the $m_{\mathrm{ES}}$ and the $\Delta E$ distributions of the reconstructed $B$ candidates are shown in Fig.s 7.14 (a), (b) and (c). Although, clear peaks are observed in the mass distributions of $\Xi_{c}$ and $\Lambda_{c}^{+}$, no peaking structure is seen in the $\left(m_{\mathrm{ES}}, \Delta E\right)$ signal region. Therefore, this is not considered as a source of significant peaking background.

\subsubsection{Background study for $B^{-} \rightarrow \Lambda_{c}^{+} \bar{\Lambda}_{c}^{-} K^{-}$Decays}

Several MC datasets and the sideband of the signal region from data are used to investigate possible background contributions. Because the phase space hardly leaves 

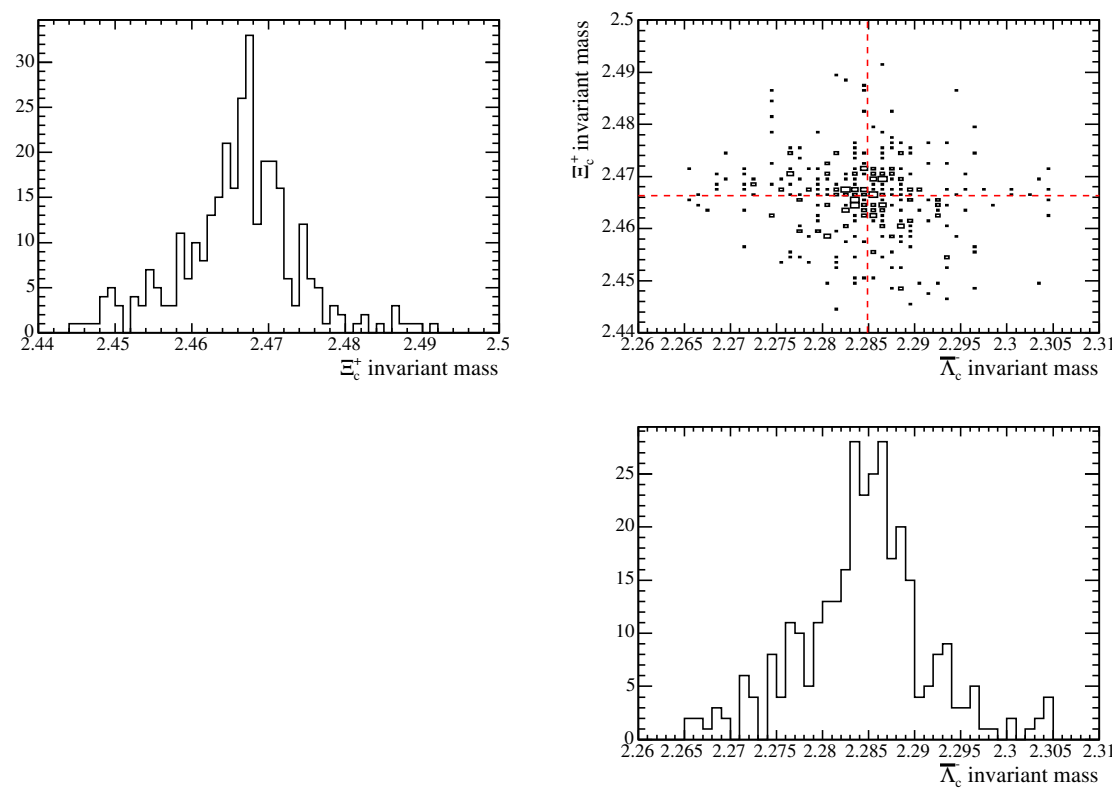

Figure 7.13: Mass distribution $\Lambda_{c}^{+}$and $\Xi_{c}$, the daughters of the $\bar{B}^{0} \rightarrow \Xi_{c}^{+} \bar{\Lambda}_{c}^{-}$reconstructed from MC dataset of $B^{0} \rightarrow \bar{\Lambda}_{c}^{-} p^{+} \pi^{-} \pi^{+}$and $\bar{B}^{0} \rightarrow \Xi_{c}^{+} \bar{\Lambda}^{0} \pi^{-}$.
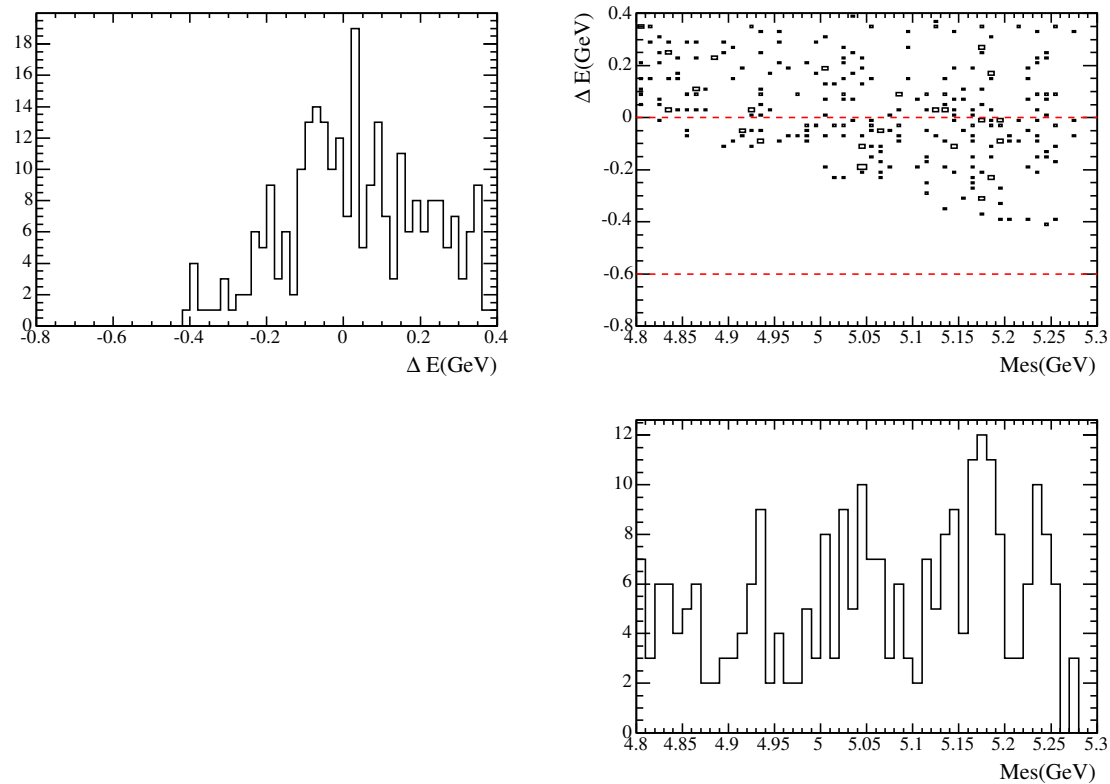

Figure 7.14: $m_{\mathrm{ES}}$ and $\Delta E$ distribution of the $\bar{B}^{0} \rightarrow \Xi_{c}^{+} \bar{\Lambda}_{c}^{-}$reconstructed from MC dataset of $B^{0} \rightarrow \bar{\Lambda}_{c}^{-} p^{+} \pi^{-} \pi^{+}$and $\bar{B}^{0} \rightarrow \Xi_{c}^{+} \bar{\Lambda}^{0} \pi^{-}$. 
any room, the feed down background contamination is negligible, and no special study is performed to estimate it. Because of the limited statistics, we do not perform a study of background from the continuum from the offpeak data.

7.3.2.1 Possible peaking background from the neutral decay $B^{0} \rightarrow \Lambda_{c}^{+} \bar{\Lambda}_{c}^{-} \bar{K}^{0}$

The neutral decay $B^{0} \rightarrow \Lambda_{c}^{+} \bar{\Lambda}_{c}^{-} \bar{K}^{0}$ could lead to a peaking background for the decay of $B^{-} \rightarrow \Lambda_{c}^{+} \bar{\Lambda}_{c}^{-} K^{-}$, where the slow $K^{0}$ is replaced by a slow $K^{-}$. To estimate the contamination from this source, 117,000 $\bar{B}^{0} \rightarrow \Lambda_{c}^{+} \bar{\Lambda}_{c}^{-} K_{s}^{0}$ events are generated as signal MC and the reconstruction and selection for $B^{-} \rightarrow \Lambda_{c}^{+} \bar{\Lambda}_{c}^{-} K^{-}$is applied. Only 269 events are observed in the signal region, as shown in Figure 7.15. When this distribution is fitted with the mean and $\sigma$ of the signal peak fixed to those measured in $B^{-} \rightarrow \Lambda_{c}^{+} \bar{\Lambda}_{c}^{-} K^{-}$events in data, a signal yield of $(23.3 \pm 15.2)$ events is seen, corresponding to an efficiency of $(0.020 \pm 0.013) \%$. Even if the mean and $\sigma$ are allowed to vary (float), the signal yield in the MC sample is only (68.8 \pm 27.6$)$ events. With an estimated efficiency $0.02 \%$ and a branching fraction $\mathcal{B}\left(\bar{B}^{0} \rightarrow \Lambda_{c}^{+} \bar{\Lambda}_{c}^{-} \bar{K}^{0}\right)=$ $7.9 \times 10^{-4}[30]$, we expect a peaking background of only 0.1 event. Compared with the statistical uncertainty for the signal events, this is negligible and can be ignored.

7.3.2.2 Peaking background from misidentification amongst the different submodes All of the five submodes of $B^{-} \rightarrow \Lambda_{c}^{+} \bar{\Lambda}_{c}^{-} K^{-}$events contain seven charged tracks. One of the two $\Lambda_{c}^{+} \mathrm{s}$ is observed in the $p K^{-} \pi^{+}$final state, and the other $\Lambda_{c}^{+}$ decays to $p K^{-} \pi^{+}, p \bar{K}^{0}$, or $\Lambda^{0} \pi^{+}$. If a $\pi^{-}$from $p \bar{K}^{0}$ or $\Lambda^{0} \pi^{+}$is misidentified as a $K^{-}$, 

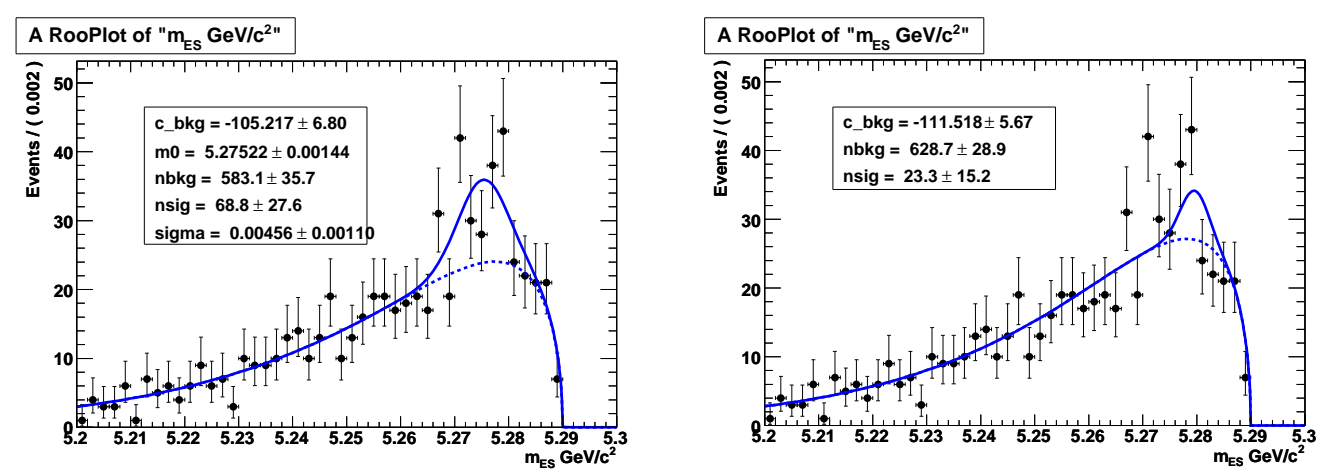

Figure 7.15: The $m_{\mathrm{ES}}$ distribution from a sample of simulated $\bar{B}^{0} \rightarrow \Lambda_{c}^{+} \bar{\Lambda}_{c}^{-} K_{s}^{0}$ events, reconstructed as $B^{-} \rightarrow \Lambda_{c}^{+} \bar{\Lambda}_{c}^{-} K^{-}$and passing all selection criteria. The same data are shown in both plots. In the left plot, the signal mean and width parameters are floated, whereas in the right plot, they are fixed to the values obtained for $B^{-} \rightarrow$ $\Lambda_{c}^{+} \bar{\Lambda}_{c}^{-} K^{-}$in data $\left(m_{0}=5.2796 \mathrm{GeV}\right.$ and $\left.\sigma=2.62 \mathrm{MeV}\right)$.

the $\Lambda_{c}^{+}$decay can be mis-reconstructed as $p K^{-} \pi^{+}$. Conversely, since, it is possible for a $p K^{-} \pi^{+}$final state to be misidentified as $p \bar{K}^{0}$ or $\Lambda^{0} \pi^{+}$. These misidentification rates are rather small because of the tight Kaon ID requirement (KLHTight), the mass window cut and the decay length cut applied to both $K_{S}^{0}$ and $\Lambda^{0}$. A detailed MC study is performed for all five submodes (117,000 events for each submode). Table 7.5 lists the number of events observed in the signal region after imposing the selection cuts for each of the five submodes in turn. (Note that these are event counts, not fitted yields.)

As shown in the Table 7.5, the only significant cross-feed happens between $B^{+}$and $B^{-}$decays with $\Lambda_{c}^{+} \rightarrow p^{+} K_{S}^{0}$ or with $\Lambda_{c}^{+} \rightarrow \Lambda^{0} \pi^{+}$. However, most of these cross-feed events are distributed broadly in $m_{\mathrm{ES}}$, as shown in Fig. 7.16, and the peaking cross-feed is small. The mis-reconstruction rates for $\left(p K \pi, p K_{S}^{0}\right)\left(p K \pi, \bar{\Lambda}^{0} \pi\right)$, 
Table 7.5: The remaining events in the $B$ signal region for each of the five modes reconstructed from each of the five signal $\mathrm{MC}$ dataset after all the selection cuts are applied to each signal modes respectively

\begin{tabular}{l|c|c|c|c|c}
\hline \hline \multirow{2}{*}{ MC dataset } & \multicolumn{5}{|c}{ Reconstructed final states } \\
\cline { 2 - 6 } & $p K \pi, p K \pi$ & $p K \pi, p K_{S}^{0}$ & $p K \pi, \bar{\Lambda}^{0} \pi$ & $p K_{S}^{0}, p K \pi$ & $\Lambda^{0} \pi, p K \pi$ \\
\hline$p K \pi, p K \pi$ & 7768 & 1 & 0 & 0 & 0 \\
\hline$p K \pi, p K_{S}^{0}$ & 11 & 9574 & 0 & 179 & 0 \\
\hline$p K \pi, \bar{\Lambda}^{0} \pi$ & 6 & 0 & 6910 & 0 & 148 \\
\hline$p K_{S}^{0}, p K \pi$ & 5 & 233 & 0 & 9563 & 0 \\
\hline$\Lambda^{0} \pi, p K \pi$ & 2 & 0 & 173 & 4 & 7064 \\
\hline
\end{tabular}

$\left(p K_{S}^{0}, p K \pi\right)$ and $\left(\bar{\Lambda}^{0} \pi, p K \pi\right)$ modes are $0.02 \%, 0.013 \%, 0.026 \%$ and $0.018 \%$, respectively. The peaking cross-feed in other sub-modes is also negligible.

\subsubsection{Possible peaking background from $b \rightarrow c \bar{u} d$ and the charmless process}

The final state $\left(p K^{-} \pi^{+} \bar{p} K^{+} \pi^{-} K^{-}\right)$can originate from $\mathrm{B}$ decays with one charmed baryon (e.g. $\left.B^{-} \rightarrow \Lambda_{c}^{+} \bar{\Delta}^{++} \phi\right)$ or even no charmed baryon (e.g. $B^{-} \rightarrow$ $\Delta^{0} \bar{\Delta}^{0}$ ). We generate two additional sets of MC events to study the background from these decays. The first sample contains charmless $B$ decays, $B^{-} \rightarrow p^{+} K^{-} \pi^{+} p^{-} K^{+} \pi^{-} K^{-}$; the other contains $B$ decays to a charmed baryon with a light anti-baryon, like $B^{-} \rightarrow \Lambda_{c}^{+} p^{-} K^{+} \pi^{-} K^{-}$. For each case, 12000 events are generated and reconstructed as $B^{-} \rightarrow \Lambda_{c}^{+} \bar{\Lambda}_{c}^{-} K^{-}$. The detection efficiencies for these modes are very low. In generic MC, where these modes are over-represented, five events from the process $B^{-} \rightarrow \Lambda_{c}^{+} p^{-} K^{+} \pi^{-}$are seen in the signal region (with high background, no peaking is visible). No event of this type is seen in the $\Lambda_{c}^{+}$mass sidebands in data. So we 

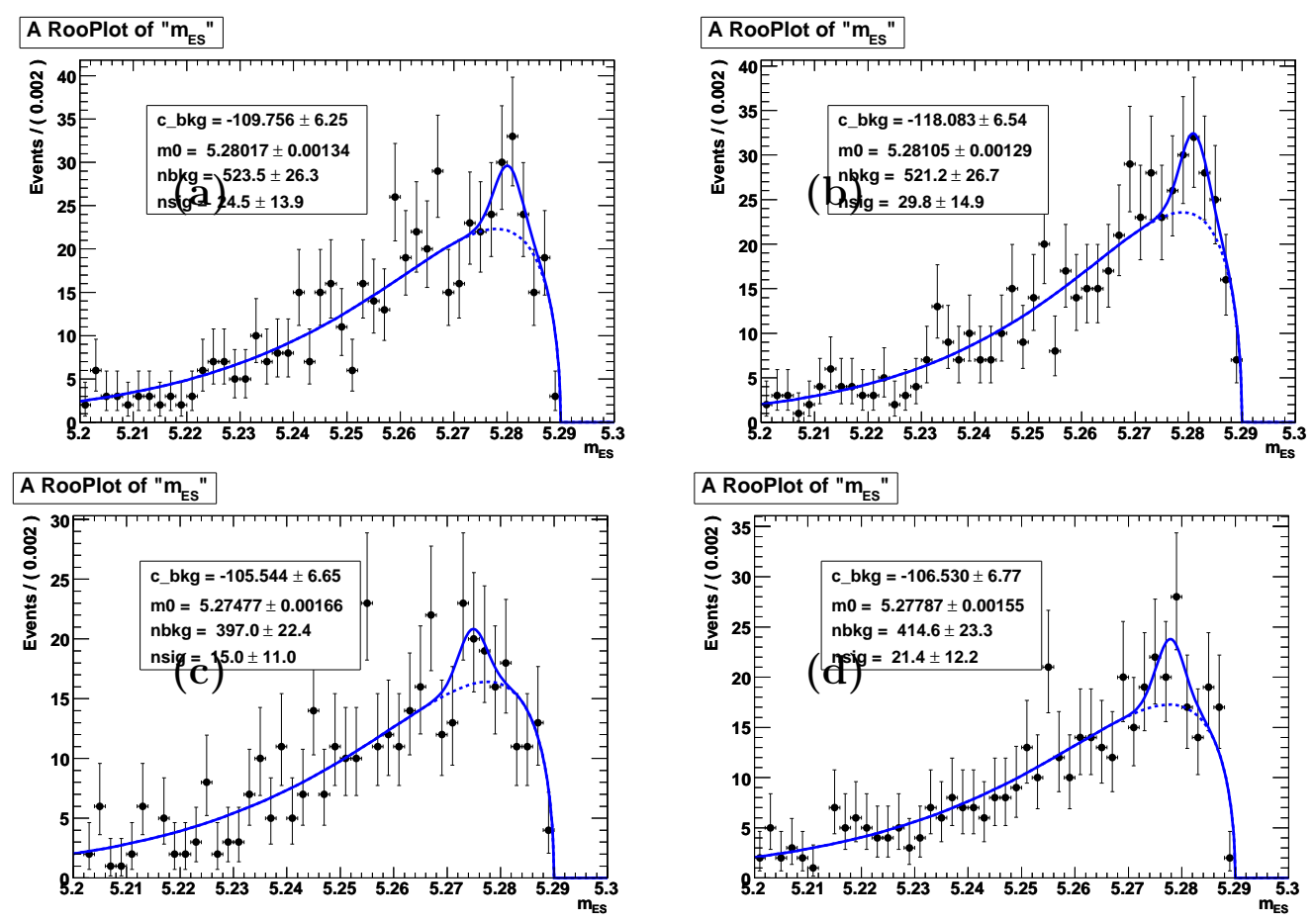

Figure 7.16: $m_{\mathrm{ES}}$ distributions of cross-feed background from the following $\mathrm{MC}$ samples: (a) mis-reconstructed $B^{-} \rightarrow \Lambda_{c}^{+}\left(p K_{S}^{0}\right) \bar{\Lambda}_{c}^{-}(p K \pi) K^{-}$candidates from the $B^{-} \rightarrow \Lambda_{c}^{+}(p K \pi) \bar{\Lambda}_{c}^{-}\left(p K_{S}^{0}\right) K^{-}$signal MC dataset, (b) mis-reconstructed $B^{-} \rightarrow$ $\Lambda_{c}^{+}(p K \pi) \bar{\Lambda}_{c}^{-}\left(p K_{S}^{0}\right) K^{-}$candidates from the $B^{-} \rightarrow \Lambda_{c}^{+}\left(p K_{S}^{0}\right) \bar{\Lambda}_{c}^{-}(p K \pi) K^{-}$signal MC dataset, (c) mis-reconstructed $B^{-} \rightarrow \Lambda_{c}^{+}\left(\Lambda^{0} \pi\right) \bar{\Lambda}_{c}^{-}(p K \pi) K^{-}$candidates from the $B^{-} \rightarrow \Lambda_{c}^{+}(p K \pi) \bar{\Lambda}_{c}^{-}\left(\bar{\Lambda}^{0} \pi\right) K^{-}$signal MC datset, (d) mis-reconstructed $B^{-} \rightarrow$ $\Lambda_{c}^{+}(p K \pi) \bar{\Lambda}_{c}^{-}\left(\bar{\Lambda}^{0} \pi\right) K^{-}$candidates from the $B^{-} \rightarrow \Lambda_{c}^{+}\left(\Lambda^{0} \pi\right) \bar{\Lambda}_{c}^{-}(p K \pi) K^{-}$signal MC datset. For each of the $m_{\mathrm{ES}}$ distribution, we fit it to Gaussian plus Argus function. 
ignore them.

\subsubsection{Study of combinatoric backgrounds}

Four generic MC samples, $B^{+} B^{-}, B^{0} \bar{B}^{0}, c \bar{c}$ and $u d s$, are used to study the combinatoric backgrounds in $B^{-} \rightarrow \Lambda_{c}^{+} \bar{\Lambda}_{c}^{-} K^{-}$. As mentioned earlier, the $B$ decays to charmed baryons is not properly implemented in the generic MC. The few signal events observed there are (accidental) results of fragmentation by Jetset. However, the combinatoric background shapes in the generic $\mathrm{MC}$ are consistent in $m_{\mathrm{ES}}$ and $\Delta E$ with those in the data, albeit with different normalizations. Figures 7.17 (a) and (b) show the $m_{\mathrm{ES}}$ and $\Delta E$ distributions of $B^{-} \rightarrow \Lambda_{c}^{+} \bar{\Lambda}_{c}^{-} K^{-}$(with both $\Lambda_{c}^{+} \rightarrow$ $p^{+} K^{-} \pi^{+}$) reconstructed and selected from the data (black point) and generic MC samples(shaded area) where all selection cuts are applied except the mass constraint, the best candidate selection, and the $m_{\mathrm{ES}}$ and $\Delta E$ cuts. Also, All signal modes present in the generic MC are removed. No peaking background is visible in the processed MC events.

Figures 7.18 (a) and (b) show the invariant mass distributions of the two $\Lambda_{c}^{+} \rightarrow$ $p^{+} K^{-} \pi^{+}$candidates (the $B$ daughters) reconstructed from data (black points) and from the generic MC (shaded area) when cuts of $m_{\mathrm{ES}}>5.2 \mathrm{GeV}$ and $|\Delta E|<0.2 \mathrm{GeV}$ are applied, and a $3 \sigma$ mass window requirement is imposed on the other $\Lambda_{c}^{+}$in the $B$ decay candidate. The background shapes agree with each other, but significant peaks are seen in both of the $\Lambda_{c}^{+}$mass distributions in data, indication additional correlated production of $\Lambda_{c}^{+} \bar{\Lambda}_{c}^{-}$. 

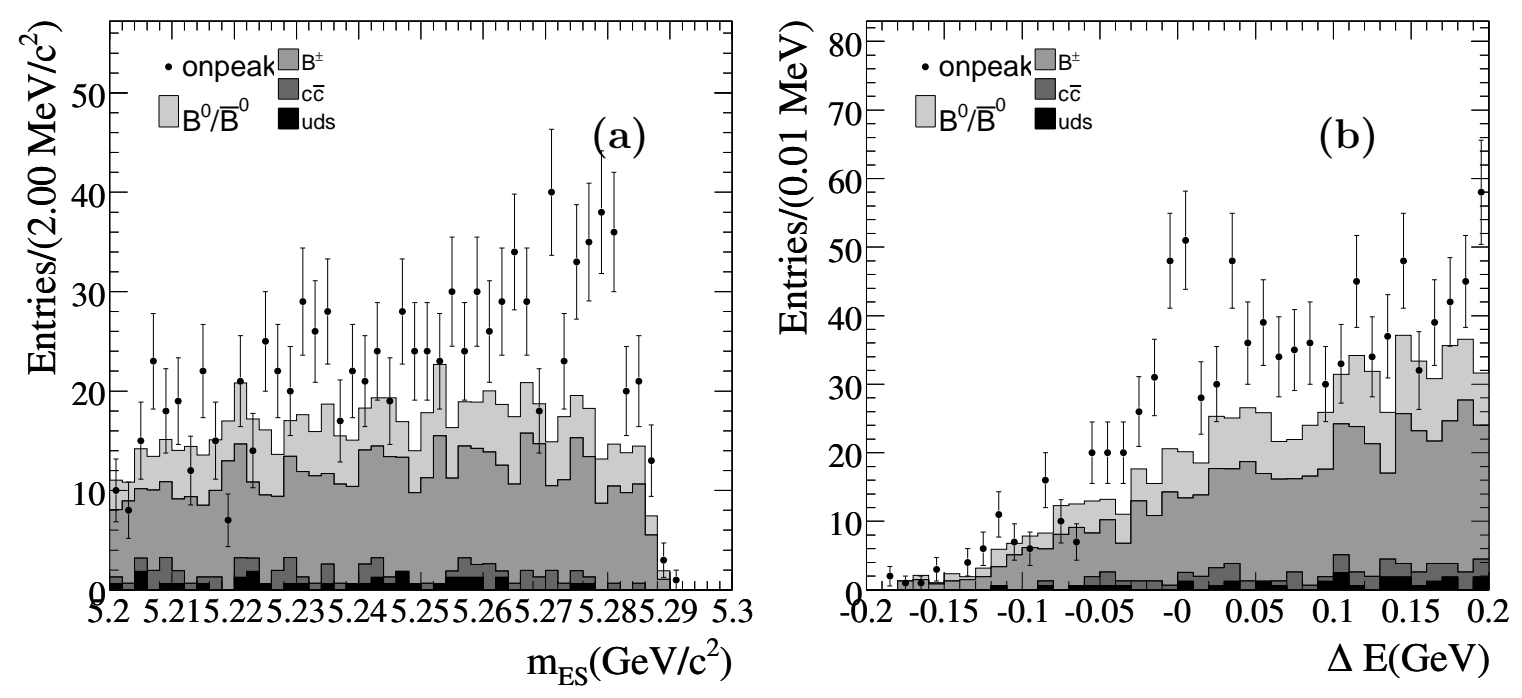

Figure 7.17: Distribution of $m_{\mathrm{ES}}$ and $\Delta E$ of $B^{-} \rightarrow \Lambda_{c}^{+} \bar{\Lambda}_{c}^{-} K^{-}\left(\Lambda_{c}^{+} \rightarrow p^{+} K^{-} \pi^{+}\right.$for both $\Lambda_{c}^{+}$) candidates for data (black point) and Generic $B \bar{B}+q \bar{q} \mathrm{MC}$ (shaded area) samples. The generic $B^{0} \bar{B}^{0}, B^{+} B^{-}, c \bar{c}$ and $u d s \mathrm{MC}$ samples are individually scaled to have luminosities equal to the data sample. (a) $m_{\mathrm{ES}}$ distribution with $|\Delta E|<0.2 \mathrm{GeV}$ (b) $\Delta E$ distribution with $m_{\mathrm{ES}}>5.20 \mathrm{GeV}$

To investigate further, the $B^{0} \bar{B}^{0}$ and $B^{+} B^{-}$generic MC samples are combined together (since both of them contain approximately equal number of events, no scale factor is applied), and fitted with an Argus function; this is shown in Figure 7.19 (a). Here, $|\Delta E|<0.03 \mathrm{GeV}$ and all other selection cuts are imposed. For comparison, the $m_{\mathrm{ES}}$ distribution of $B^{-} \rightarrow \Lambda_{c}^{+} \bar{\Lambda}_{c}^{-} K^{-}$candidates reconstructed from the $\Delta E$ sideband region are also fitted with an Argus function, as shown in Fig 7.19(b). The shape is very similar to the one from generic MC, with no peaking background observed.

Figures 7.20 show the $p^{*}$ (the momentum in the beam-rest frame) distributions 

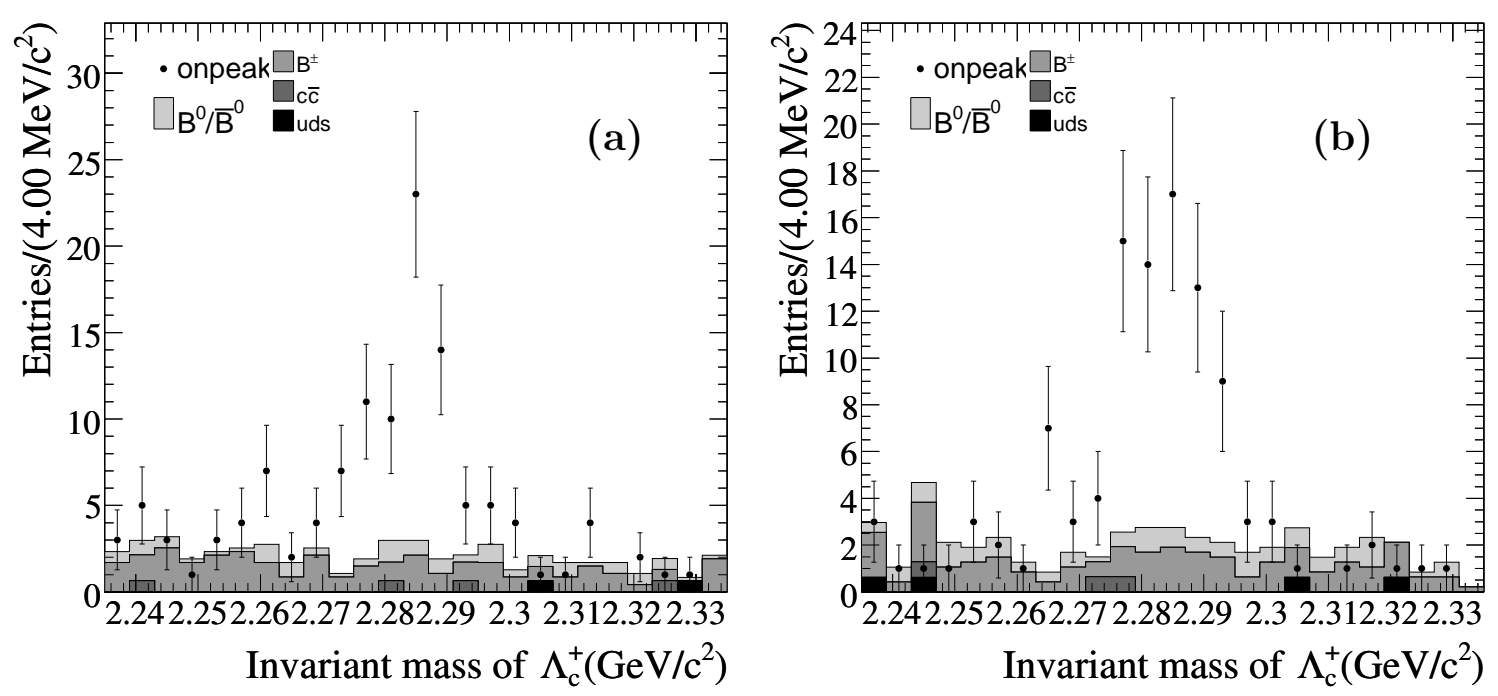

Figure 7.18: Mass distribution of (a) the first $\Lambda_{c}^{+}$and (b) the second $\Lambda_{c}^{+}$of $B^{-} \rightarrow$ $\Lambda_{c}^{+} \bar{\Lambda}_{c}^{-} K^{-}\left(\Lambda_{c}^{+} \rightarrow p^{+} K^{-} \pi^{+}\right.$for both $\left.\Lambda_{c}^{+}\right)$for data and Generic MC samples where we require $m_{\mathrm{ES}}>5.2 \mathrm{GeV}$ and $|\Delta E|<0.2 \mathrm{GeV}$ and we make $3 \sigma_{m}$ mass cut for the other $\Lambda_{c}^{+}$when we plot one $\Lambda_{c}^{+}$mass distribution. The generic $B^{0} \bar{B}^{0}, B^{+} B^{-}, c \bar{c}$ and $u d s$ $\mathrm{MC}$ samples are individually scaled to have luminosities equal to the data sample.

of the two $\Lambda_{c}^{+}$decaying from the $B^{-} \rightarrow \Lambda_{c}^{+} \bar{\Lambda}_{c}^{-} K^{-}$mode, where both $\Lambda_{c}^{+}$baryons decay into $p K \pi$. The reconstructed $p^{*}$ of $B \bar{B}, q \bar{q}$, and data all peak in the $(0,1 \mathrm{GeV})$ region. This indicates that after imposing all kinematic cuts on the $B^{-} \rightarrow \Lambda_{c}^{+} \bar{\Lambda}_{c}^{-} K^{-}$ candidates, the system is almost fully constrained and the $p^{*}$ distribution carries little additional information to distinguish between background from $B$ decays or from the continuum.

Other event shape variables like thrust, $\mathrm{R} 2$ and $\cos ^{*} \Theta_{B}$ (the polar angle of $B$ candidate in the beam rest frame) are also studied, as shown in Figures 7.21. 

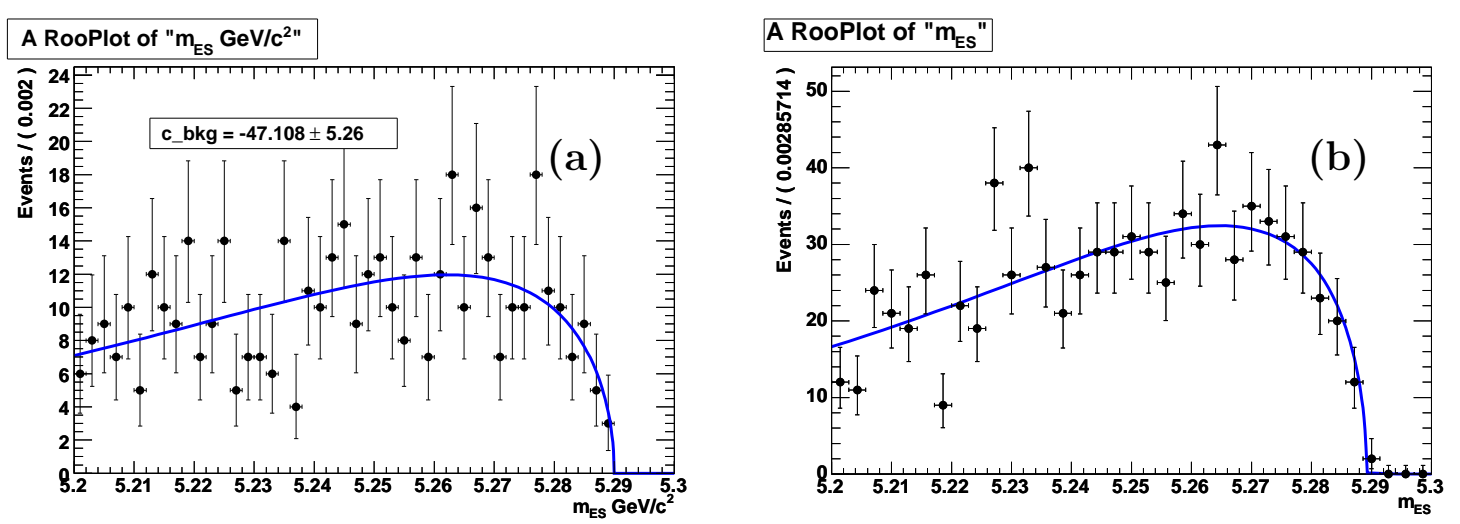

Figure 7.19: $m_{\mathrm{ES}}$ distribution of $B^{-} \rightarrow \Lambda_{c}^{+} \bar{\Lambda}_{c}^{-} K^{-}$(both $\Lambda_{c}^{+} \rightarrow p^{+} K^{-} \pi^{+}$) candidate fitted with an Argus function, In (a), events from the combined $B^{0} \bar{B}^{0}$ and $B^{+} B^{-}$ generic MC samples are shown with a cut of $|\Delta E|<0.03$. In (b), events from the $\Delta E$ sideband of data are shown $\left(m_{E S}>5.2 \mathrm{GeV}\right.$ and $\left.|\Delta E|>0.05 \mathrm{GeV}\right)$. The normalization in (a) is not scale to match the data integrated luminosity.
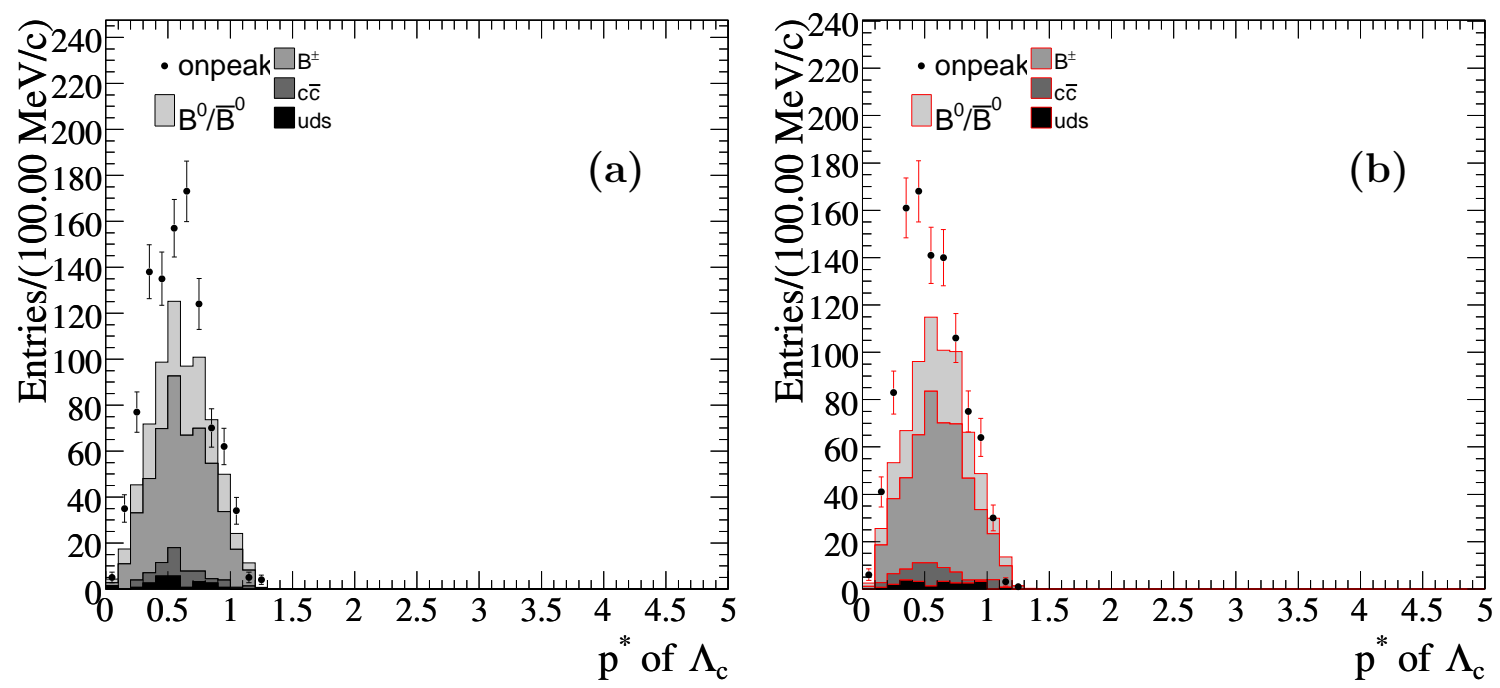

Figure 7.20: $p^{*}$ distribution of (a) the first $\Lambda_{c}^{+}$and (b) the second $\Lambda_{c}^{+}$of $B^{-} \rightarrow$ $\Lambda_{c}^{+} \bar{\Lambda}_{c}^{-} K^{-}\left(\Lambda_{c}^{+} \rightarrow p^{+} K^{-} \pi^{+}\right.$for both $\left.\Lambda_{c}^{+}\right)$for data and Generic MC samples where the $m_{\mathrm{ES}}>5.20 \mathrm{GeV}$ and $|\Delta E|<0.2 \mathrm{GeV}$. The generic $B^{0} \bar{B}^{0}, B^{+} B^{-}, c \bar{c}$ and $u d s \mathrm{MC}$ samples are individually scaled to have luminosities equal to the data sample. 
Because the backgrounds from continuum events are far less than backgrounds from $B \bar{B}$ decays, the event shape variable cuts are not necessary.
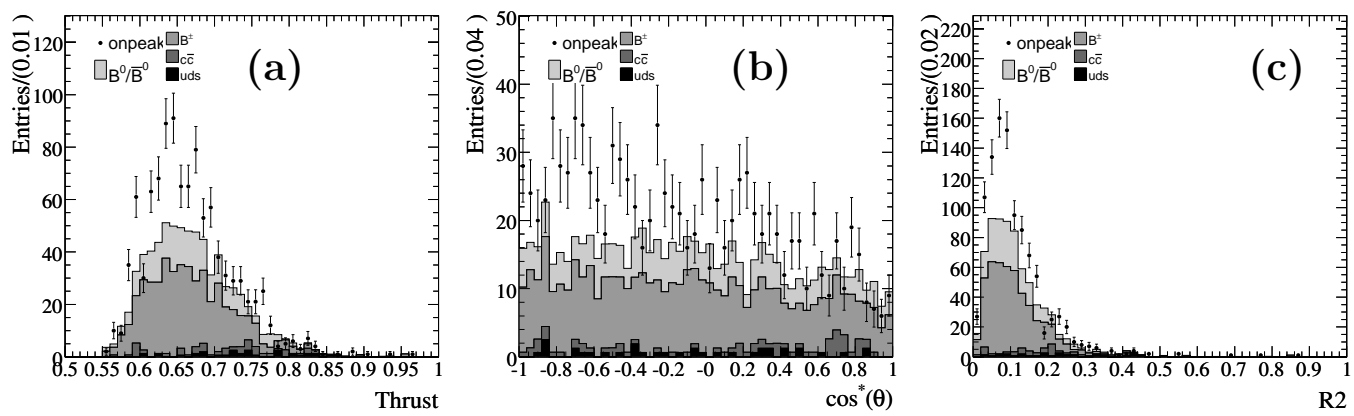

Figure 7.21: Distribution of (a) thrust (b) $\cos ^{*} \Theta_{B}$ and (c) R2 of $B^{-} \rightarrow \Lambda_{c}^{+} \bar{\Lambda}_{c}^{-} K^{-}$ $\left(\Lambda_{c}^{+} \rightarrow p^{+} K^{-} \pi^{+}\right.$for both $\left.\Lambda_{c}^{+}\right)$for data and generic MC samples where $m_{\mathrm{ES}}>5.20 \mathrm{GeV}$ and $|\Delta E|<0.2 \mathrm{GeV}$. The generic $B^{0} \bar{B}^{0}, B^{+} B^{-}, c \bar{c}$ and $u d s \mathrm{MC}$ samples are individually scaled to have integrated luminosities equal to the data sample.

\subsection{Estimation of the systematic uncertainties}

\subsubsection{B counting systematics}

The B-counting method, described in detail in [56], is used to determine the total number of $\Upsilon(4 S)$ produced in the (on-peak) data. Basically, it assumes that the increase in the ratio of hadronic events and $\mu \bar{\mu}$ pairs between on-peak and off-peak data results from the $\Upsilon(4 S)$ meson production; the hadronic decay rates of the $\Upsilon(4 S)$ than into $\mu \bar{\mu}$ is much higher than those from the continuum. Since these ratios are well-measured, the number of $\Upsilon(4 S) \rightarrow B \bar{B}$ can be obtained by counting the number of muon pairs and those of the hadronic events. The systematic error quoted for 
Run $1-4$ is $1.1 \%$. Here, the number of $\Upsilon(4 S) \rightarrow B^{0} \bar{B}^{0}$ is assumed to be equal to the number of $\Upsilon(4 S) \rightarrow B^{+} B^{-}$.

\subsubsection{Tracking efficiency}

The uncertainty on the track reconstruction efficiency is obtained according to the recipe provided by the Tracking Efficiency Task Force [51]. All the charged tracks $(p, K, \pi)$ are derived from the ChargedTracks list, and a flat correction of $(0.25 \pm 1.4) \%$ is assigned for each track. For the decay modes in this analysis, a total of seven charged tracks is present in each charged $B$ decay, and eight in each neutral $B$ decay. As these errors are not random, they are added linearly, resulting in a systematic uncertainty of $(1.4) \% \times 7($ or 8$)=9.8(11.2) \%$ for a decay mode.

An extra uncertainty $2 \%$, estimated from a $K_{S}^{0}$ study [51], is added for $K_{S}^{0} \rightarrow$ $\pi^{+} \pi^{-}$decays because of its long decay length to cover any possible inaccuracies in the MC simulation for the $K_{S}^{0}$ 's not originating from the beam spot. The same recipe is also used to evaluate the effect on the $\Lambda^{0}$ decay from a study of inclusive $\Xi_{c}^{0}$ production [27], and a similar uncertainty of $2 \%$ is used. Table 7.4 .2 summarizes these uncertainties for each decay mode.

\subsubsection{Particle identification}

At BABAR, particle identification is performed by using $\mathrm{dE} / \mathrm{dx}$ information from the tracking systems (DCH and SVT), and Cheronkov photon information from the DIRC. In the simulation, the detector response is modeled with GEANT4. The overall agreement is good, but the efficiency and purity of some PID selectors are seen 
Table 7.6: Summary of correction factors and uncertainties on the efficiency from tracking

\begin{tabular}{llcc}
\hline \hline$B$ decay & $\Lambda_{c}^{+}\left(\Xi_{c}\right)$ decay & correction(\%) & uncertainty(\%) \\
\hline \multirow{2}{*}{$B^{-} \rightarrow \Xi_{c}^{0} \bar{\Lambda}_{c}^{-}$} & $\Xi \pi, p K \pi$ & 98.26 & 10.0 \\
\cline { 2 - 4 } $\bar{B}^{0} \rightarrow \Xi_{c}^{+} \bar{\Lambda}_{c}^{-}$ & $\Xi \pi \pi, p K \pi$ & 98.26 & 10.0 \\
\hline & $p K \pi, p K \pi$ & 98.02 & 11.4 \\
\cline { 2 - 4 }$B^{-} \rightarrow \Lambda_{c}^{+} \bar{\Lambda}_{c}^{-} K^{-}$ & $p K \pi, p \bar{K}^{0}$ & 98.26 & 9.8 \\
\cline { 2 - 4 } & $p K \pi, \Lambda \pi$ & 98.26 & 10.0 \\
\cline { 2 - 4 } & $p \bar{K}^{0}, p K \pi$ & 98.26 & 10.0 \\
\cline { 2 - 4 } & $\Lambda \pi, p K \pi$ & 98.26 & 10.0 \\
\hline $\bar{B}^{0} \rightarrow \Lambda_{c}^{+} \bar{\Lambda}_{c}^{-} K_{s}^{0}$ & $p K \pi, p K \pi$ & 98.26 & 11.4 \\
\hline
\end{tabular}

to differ between data and simulation at the level of a few percent. To correct for the discrepancy between data and simulation, high purity control samples are used to compute the ratio of efficiencies in data and simulation in bins of track momentum and polar angle. The correction is applied to signal MC samples on a track-by-track basis using a randomized process known as "PID tweaking" [52]. Due to limited statistics in the control samples and to other effects not taken into account (e.g. correlations between tracks) this correction has an associated systematic uncertainty. The PID uncertainties on our branching fraction measurements are estimated by performing the whole analysis with and without PID tweaking in the signal MC. The relative difference in signal selection efficiencies is shown in table 7.4.3. We take this difference as the systematic uncertainty for each mode. The final results are quoted with the PID tweaking corrections applied. 
Table 7.7: Systematic uncertainty due to the PID

\begin{tabular}{|c|c|c|}
\hline$B$ decay & $\Lambda_{c}^{+}\left(\Xi_{c}\right)$ decay & error $(\%)$ \\
\hline \multirow{2}{*}{$B^{-} \rightarrow \Xi_{c}^{0} \bar{\Lambda}_{c}^{-}$} & $\Xi \pi, p K \pi$ & 0.16 \\
\hline & $\Lambda^{0} K \pi, p K \pi$ & 1.34 \\
\hline $\bar{B}^{0} \rightarrow \Xi_{c}^{+} \bar{\Lambda}_{c}^{-}$ & $\Xi \pi \pi, p K \pi$ & 0.38 \\
\hline \multirow{5}{*}{$B^{-} \rightarrow \Lambda_{c}^{+} \bar{\Lambda}_{c}^{-} K^{-}$} & $p K \pi, p K \pi$ & 1.2 \\
\hline & $p K \pi, p \bar{K}^{0}$ & 0.12 \\
\hline & $p K \pi, \Lambda \pi$ & 0.17 \\
\hline & $p \bar{K}^{0}, p K \pi$ & 0.24 \\
\hline & $\Lambda \pi, p K \pi$ & 0.5 \\
\hline$\overline{\bar{B}}^{0} \rightarrow \Lambda_{c}^{+} \bar{\Lambda}_{c}^{-} K_{s}^{0}$ & $p K \pi, p K \pi$ & 0.24 \\
\hline
\end{tabular}

\subsubsection{Monte Carlo statistics}

The Monte Carlo samples used to evaluate the efficiencies have finite statistics. The statistical uncertainty from this is considered as a source of systematic uncertainty and is estimated to be between 1 and $2 \%$ as shown in Table 7.1.

\subsubsection{Effect of intermediate resonances in $\Lambda_{c}^{+}$decays}

The effects of intermediate resonances in $\Lambda_{c}^{+}$decays are explored in section 7.1.4, and any contribution in the systematic uncertainty from this is ignored as explained before.

\subsubsection{Uncertainties in the decay branching fractions}

In the analysis we consistently use the $\mathcal{B}\left(K_{s}^{0} \rightarrow \pi^{+} \pi^{-}\right), \mathcal{B}\left(\Xi^{-} \rightarrow \Lambda^{0} \pi^{-}\right)$, and $\mathcal{B}\left(\Lambda_{c}^{+} \rightarrow p^{+} K^{-} \pi^{+}\right)$branching fractions listed in the 2006 PDG, as well the listed values for $\Gamma\left(\Lambda^{0} K^{-} \pi^{+}\right) / \Gamma\left(\Xi^{-} \pi^{+}\right), \Gamma\left(p \bar{K}^{0}\right) / \Gamma\left(p^{+} K^{-} \pi^{+}\right)$and $\Gamma\left(\Lambda^{0} \pi^{-}\right) / \Gamma\left(p^{+} K^{-} \pi^{+}\right)$. Ta- 
ble 7.8 lists the systematic uncertainties arising from the uncertainties listed in these values in the PDG 2006.

\subsubsection{The difference in $\Delta E$ between data and $\mathrm{MC}$}

The mean and the $\sigma$ of the $\Delta E$ distributions observed from data are different from those in the $\mathrm{MC}$, but the efficiency of the $\Delta E$ cut is decided from signal $\mathrm{MC}$ only. Because we don't have enough statistics in data to perform a complete study, we use the comparison study from $B \rightarrow D \bar{D} K$, and apply an uncertainty of $3 \%$ for the $\Delta E$ cut. (The effect is small since the $\Delta E$ cut window is substantially wider than the $\Delta E$ resolution.)

\subsubsection{Maximum likelihood fits and signal parameterizations}

Some of the fit parameters are fixed at the values obtained from the MC during the fit process in the data, and are varied by their respective errors; the difference in the fitted yield before and after is considered as a systematic uncertainty.

\subsubsection{Summary of systematics uncertainties}

The relative systematic uncertainties in the branching fraction measurement for all $B^{-} \rightarrow \Lambda_{c}^{+} \bar{\Lambda}_{c}^{-} K^{-}$, all $B^{-} \rightarrow \Xi_{c}^{0} \bar{\Lambda}_{c}^{-}$, and all $B^{0}$ decay modes are summarized in Table 7.4.9, Table 7.10, and Table 7.4.9, respectively. Following the standard error propagation method, the individual contribution to the systematic uncertainties are added in quadrature. The uncertainties for each of the individual $\mathcal{B}\left(\Lambda_{c}^{+} \rightarrow p^{+} K^{-} \pi^{+}\right)$ decay modes are listed. 
Table 7.8: Summary of uncertainties from the known decay branching fractions(\%) and corrections(\%)

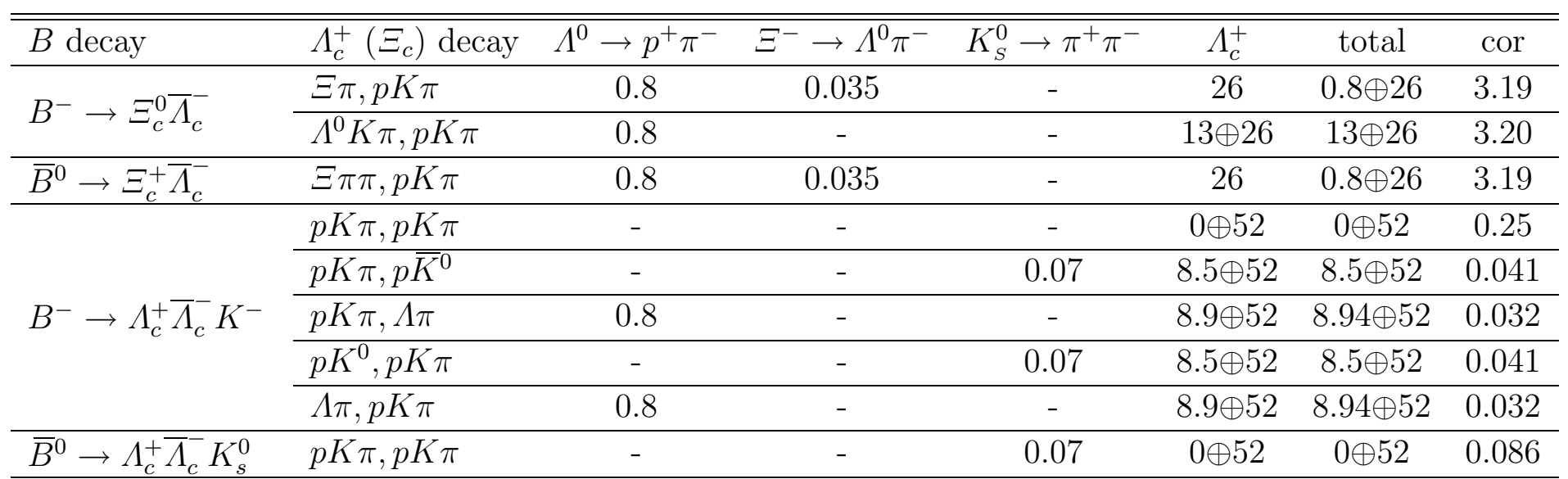


Table 7.9: Summary of the relative systematic uncertainties(\%) of $B^{-} \rightarrow \Lambda_{c}^{+} \bar{\Lambda}_{c}^{-} K^{-}$ decays

\begin{tabular}{lccccc}
\hline \hline systematic resource & $p K \pi, p K \pi$ & $p K \pi, p K^{0}$ & $p \bar{K}^{0}, p K \pi$ & $p K \pi \bar{\Lambda}^{0} \pi$ & $\Lambda^{0} \pi, p K \pi$ \\
\hline$B$ counting & 1.1 & 1.1 & 1.1 & 1.1 & 1.1 \\
Tracking & 9.8 & 10.0 & 10.0 & 10.0 & 10.0 \\
PID & 1.2 & 0.12 & 0.24 & 0.17 & 0.5 \\
MC statistics & 1.20 & 1.04 & 1.04 & 1.22 & 1.23 \\
$\Delta E$ cut & 3 & 3 & 3 & 3 & 3 \\
$\mathcal{B}$ of itermediate state & $\oplus 52$ & $8.5 \oplus 52$ & $8.5 \oplus 52$ & $8.94 \oplus 52$ & $8.94 \oplus 52$ \\
Fit related & 1.15 & 0.26 & 1.09 & 1.11 & 0.1 \\
\hline total & $10.5 \oplus 52$ & $13.55 \oplus 52$ & $13.59 \oplus 52$ & $13.89 \oplus 52$ & $13.85 \oplus 52$ \\
\hline
\end{tabular}

Table 7.10: Summary of the relative systematic uncertainties(\%) of $B^{-} \rightarrow \Xi_{c}^{0} \bar{\Lambda}_{c}^{-}$ decays

\begin{tabular}{lcc}
\hline \hline systematic resource & $\Xi \pi, p K \pi$ & $\Lambda^{0} K \pi, p K \pi$ \\
\hline$B$ counting & 1.1 & 1.1 \\
Tracking & 10.0 & 10.0 \\
PID & 0.16 & 1.34 \\
MC statistics & 2.24 & 2.22 \\
$\Delta E$ cut & 3 & 3 \\
$\mathcal{B}$ of itermediate state & $0.8 \oplus 26$ & $0.8(13) \oplus 26$ \\
Fit related & 1 & 1 \\
\hline total & $10.7 \oplus 26$ & $10.8(16.9) \oplus 26$ \\
\hline
\end{tabular}


Table 7.11: Summary of the relative systematic uncertainties(\%) of $\bar{B}^{0} \rightarrow \Xi_{c}^{+} \bar{\Lambda}_{c}^{-}$and $\bar{B}^{0} \rightarrow \Lambda_{c}^{+} \bar{\Lambda}_{c}^{-} K_{s}^{0}$ decays

\begin{tabular}{lcc}
\hline \hline systematic resource & $\bar{B}^{0} \rightarrow \Xi_{c}^{+} \bar{\Lambda}_{c}^{-}$ & $\bar{B}^{0} \rightarrow \Lambda_{c}^{+} \bar{\Lambda}_{c}^{-} K_{s}^{0}$ \\
\hline$B$ counting & 1.1 & 1.1 \\
Tracking & 11.4 & 11.4 \\
PID & 0.38 & 0.24 \\
MC statistics & 2.36 & 1.46 \\
$\Delta E$ cut & 3 & 3 \\
$\mathcal{B}$ of itermediate state & $0.8 \oplus 26$ & $0.07 \oplus 52$ \\
Fit related & 3.5 & 2.5 \\
\hline total & $12.58 \oplus 26$ & $12.19 \oplus 52$ \\
\hline
\end{tabular}




\section{CHAPTER 8 \\ RESULTS AND DISCUSSION}

\subsection{Branching fraction calculation}

The branching fractions of the $\bar{B} \rightarrow \Xi_{c} \bar{\Lambda}_{c}^{-}$decays and the $B^{-} \rightarrow \Lambda_{c}^{+} \bar{\Lambda}_{c}^{-} \bar{K}$ decays are calculated differently. No measurement for an absolute branching fraction of either $\Xi_{c}^{0}$ or $\Xi_{c}^{+}$exists; hence the product branching faction: $\mathcal{B}\left(B \rightarrow \Xi_{c} \bar{\Lambda}_{c}^{-}\right) \times \mathcal{B}\left(\Xi_{c}\right)$, where $\mathcal{B}\left(\Xi_{c}\right)$ represents either $\mathcal{B}\left(\Xi_{c}^{0} \rightarrow \Xi^{-} \pi^{+}\right)$or $\mathcal{B}\left(\Xi_{c}^{+} \rightarrow \Xi^{-} \pi^{+} \pi^{+}\right)$is quoted. For

the $B^{-} \rightarrow \Lambda_{c}^{+} \bar{\Lambda}_{c}^{-} \bar{K}$ decays, the absolute branching fractions are quoted, since all the $\Lambda_{c}^{+}$branching-fractions are already known.

$$
\text { 8.1.1 } \bar{B} \rightarrow \Xi_{c} \bar{\Lambda}_{c}^{-} \text {decays }
$$

The branching fraction of the $i$ th decay mode can be calculated as

$$
\mathcal{B}\left(\bar{B} \rightarrow \Xi_{c} \Lambda_{c}^{+}\right) \mathcal{B}_{i}\left(\Xi_{c}\right)=\frac{N_{\mathrm{Sig}, i}}{f_{i} \cdot \epsilon_{i} \cdot \mathcal{B}\left(\Lambda_{c}^{+} \rightarrow p^{+} K^{-} \pi^{+}\right) \cdot N_{B \bar{B}}}
$$

where $N_{B \bar{B}}$ is the number of $B \bar{B}$ events $\left(B^{0} \bar{B}^{0}\right.$ and $B^{+} B^{-}$together $)$, and $N_{\mathrm{Sig}, i}$ is the measured signal yield of the $i$ th decay channel from the fit, $\epsilon_{i}$ is the reconstruction and selection efficiency for the $i$ th decay mode, $f_{i}$ is the correction factor for the $i$ th decay mode: a product of the tracking correction factor, and any additional branching fraction, e.g. $\mathcal{B}\left(\Xi^{-} \rightarrow \Lambda^{0} \pi^{-}\right)$and $\mathcal{B}\left(\Lambda^{0} \rightarrow p^{+} \pi^{-}\right)$. (Here, we assume $\left.\Gamma\left(\Upsilon(4 S) \rightarrow B^{+} B^{-}\right) / \Gamma\left(\Upsilon(4 S) \rightarrow B^{0} \bar{B}^{0}\right)=1.\right)$

We obtain the following results:

$$
\mathcal{B}\left(B^{-} \rightarrow \Xi_{c}^{0} \bar{\Lambda}_{c}^{-}\right) \times \mathcal{B}\left(\Xi_{c}^{0} \rightarrow \Xi^{-} \pi^{+}\right)=(1.91 \pm 0.77 \pm 0.20 \pm 0.49) \times 10^{-5}
$$




$$
\begin{gathered}
\mathcal{B}\left(B^{-} \rightarrow \Xi_{c}^{0} \bar{\Lambda}_{c}^{-}\right) \times \mathcal{B}\left(\Xi_{c}^{0} \rightarrow \Lambda^{0} K^{-} \pi^{+}\right)=(2.46 \pm 1.02 \pm 0.26 \pm 0.64) \times 10^{-5} \\
\mathcal{B}\left(\bar{B}^{0} \rightarrow \Xi_{c}^{+} \bar{\Lambda}_{c}^{-}\right) \times \mathcal{B}\left(\Xi_{c}^{+} \rightarrow \Xi^{-} \pi^{+} \pi^{+}\right)=(1.50 \pm 1.07 \pm 0.19 \pm 0.39) \times 10^{-5}
\end{gathered}
$$

where the uncertainties listed are statistical, systematic, and from the absolute branching fraction of $\Lambda^{0}$, respectively.

For reference purpose, the following product branching fractions measured are also quoted:

$$
\begin{aligned}
\mathcal{B}\left(B^{-} \rightarrow \Xi_{c}^{0} \bar{\Lambda}_{c}^{-}\right) \cdot \mathcal{B}\left(\Xi_{c}^{0} \rightarrow \Xi^{-} \pi^{+}\right) \cdot \mathcal{B}\left(\Lambda_{c}^{+} \rightarrow p^{+} K^{-} \pi^{+}\right) & =(9.55 \pm 3.85 \pm 1.00) \times 10^{-7} \\
\mathcal{B}\left(B^{-} \rightarrow \Xi_{c}^{0} \bar{\Lambda}_{c}^{-}\right) \cdot \mathcal{B}\left(\Xi_{c}^{0} \rightarrow \Lambda^{0} K^{-} \pi^{+}\right) \cdot \mathcal{B}\left(\Lambda_{c}^{+} \rightarrow p^{+} K^{-} \pi^{+}\right) & =(12.30 \pm 5.10 \pm 3.20) \times 10^{-7} \\
\mathcal{B}\left(\bar{B}^{0} \rightarrow \Xi_{c}^{+} \bar{\Lambda}_{c}^{-}\right) \cdot \mathcal{B}\left(\Xi_{c}^{+} \rightarrow \Xi^{-} \pi^{+} \pi^{+}\right) \cdot \mathcal{B}\left(\Lambda_{c}^{+} \rightarrow p^{+} K^{-} \pi^{+}\right) & =(7.5 \pm 5.35 \pm 0.95) \times 10^{-7}
\end{aligned}
$$

where the uncertainties are statistical and systematic, respectively.

$$
\text { 8.1.2 } \bar{B} \rightarrow \Lambda_{c}^{+} \bar{\Lambda}_{c}^{-} \bar{K} \text { decays }
$$

The $\mathcal{B}\left(B^{-} \rightarrow \Lambda_{c}^{+} \bar{\Lambda}_{c}^{-} K^{-}\right)$for the $i$ th final state, $\mathcal{B}_{i}\left(B^{-} \rightarrow \Lambda_{c}^{+} \bar{\Lambda}_{c}^{-} K^{-}\right)$, is given

by

$$
\mathcal{B}_{i}\left(B^{-} \rightarrow \Lambda_{c}^{+} \bar{\Lambda}_{c}^{-} K^{-}\right)=\frac{N_{\mathrm{Sig}, \mathrm{i}}}{f_{i} \cdot \epsilon_{i} \cdot \mathcal{B}^{2}\left(\Lambda_{c}^{+} \rightarrow p^{+} K^{-} \pi^{+}\right) \cdot N_{B \bar{B}}},
$$

where index $i=1, \ldots 5$, represents the different decay modes in the list of $B^{-} \rightarrow$ $\left(p K^{-} \pi^{+}\right)\left(p^{-} K^{+} \pi^{-}\right) K^{-},\left(p K^{-} \pi^{+}\right)\left(p^{-} K^{0}\right) K^{-},\left(p \overline{K^{0}}\right)\left(p^{-} K^{+} \pi^{-}\right) K^{-},\left(p K^{-} \pi^{+}\right)\left(\bar{\Lambda}^{0} \pi^{-}\right) K^{-}$, and $\left(\Lambda^{0} \pi^{+}\right)\left(p^{-} K^{+} \pi^{-}\right) K^{-}$. The definitions of the variables $N_{s i g, i}, \epsilon_{i}$ and $N_{B \bar{B}}$ are the same as in equation 8.1, and $f_{i}$ is the product of the correction factor for tracking, the branching fraction of the hyperon branching fractions $\left(\Lambda^{0}, K_{S}^{0}\right)$ and the $\Lambda_{c}^{+}$ 
branching fraction for the $i$ th decay channel relative to $\mathcal{B}\left(\Lambda_{c}^{+} \rightarrow p^{+} K^{-} \pi^{+}\right)$. The five independent measurements of $\mathcal{B}\left(B^{-} \rightarrow \Lambda_{c}^{+} \bar{\Lambda}_{c}^{-} K^{-}\right)$are :

$$
\begin{aligned}
& \mathcal{B}_{1}\left(B^{-} \rightarrow \Lambda_{c}^{+} \bar{\Lambda}_{c}^{-} K^{-}\right)=(1.16 \pm 0.21 \pm 0.12 \pm 0.60) \times 10^{-3} \\
& \mathcal{B}_{2}\left(B^{-} \rightarrow \Lambda_{c}^{+} \bar{\Lambda}_{c}^{-} K^{-}\right)=(1.92 \pm 0.53 \pm 0.26 \pm 1.00) \times 10^{-3} \\
& \mathcal{B}_{3}\left(B^{-} \rightarrow \Lambda_{c}^{+} \bar{\Lambda}_{c}^{-} K^{-}\right)=(1.51 \pm 0.49 \pm 0.21 \pm 0.79) \times 10^{-3} \\
& \mathcal{B}_{4}\left(B^{-} \rightarrow \Lambda_{c}^{+} \bar{\Lambda}_{c}^{-} K^{-}\right)=(0.60 \pm 0.43 \pm 0.08 \pm 0.31) \times 10^{-3} \\
& \mathcal{B}_{5}\left(B^{-} \rightarrow \Lambda_{c}^{+} \bar{\Lambda}_{c}^{-} K^{-}\right)=(0.58 \pm 0.47 \pm 0.08 \pm 0.30) \times 10^{-3}
\end{aligned}
$$

where the uncertainties are statistical, systematic, and from the absolute $\Lambda_{c}^{+}$branching fraction, respectively.

Furthermore, the branching fraction of $\bar{B}^{0} \rightarrow \Lambda_{c}^{+} \bar{\Lambda}_{c}^{-} \bar{K}^{0}$ is obtained as

$$
\mathcal{B}\left(\bar{B}^{0} \rightarrow \Lambda_{c}^{+} \bar{\Lambda}_{c}^{-} \bar{K}^{0}\right)=(0.40 \pm 0.33 \pm 0.05 \pm 0.21) \times 10^{-3}
$$

where the uncertainties listed are the same as before.

\subsubsection{Combining the branching fractions}

Finally, the branching fraction results measured from the different decay final states are combined. Two different methods are applied to calculate the combined branching fractions and are described here. 


\subsubsection{Method 1}

From equation 8.5, the total measured $B^{-} \rightarrow \Lambda_{c}^{+} \bar{\Lambda}_{c}^{-} K^{-}$signal $N_{\text {total }}$ is obtained

as

$$
\begin{aligned}
N_{\text {total }} & =\sum_{i=1}^{5} N_{\text {sig }, i} \\
& =\sum_{i=1}^{5} N_{B \bar{B}} \cdot \mathcal{B}_{i}\left(B^{-} \rightarrow \Lambda_{c}^{+} \bar{\Lambda}_{c}^{-} K^{-}\right) \cdot \mathcal{B}^{2}\left(\Lambda_{c}^{+} \rightarrow p^{+} K^{-} \pi^{+}\right) \cdot f_{i} \cdot \epsilon_{i}
\end{aligned}
$$

where $N_{s i g, i}$ is the fitted yields of the $i$ th final state $(\mathrm{i}=1, \ldots 5), \epsilon_{i}$ is the efficiency of the $i$ th decay mode and $f_{i}$ is the product of branching fractions with other correction factors described earlier. Since the results of the branching fraction of $B^{-} \rightarrow \Lambda_{c}^{+} \bar{\Lambda}_{c}^{-} K^{-}$ obtained from different final states should be consistent, using $\mathcal{B}_{i}\left(B^{-} \rightarrow \Lambda_{c}^{+} \bar{\Lambda}_{c}^{-} K^{-}\right)=$ $\mathcal{B}\left(B^{-} \rightarrow \Lambda_{c}^{+} \bar{\Lambda}_{c}^{-} K^{-}\right)$, we get

$$
\mathcal{B}\left(B^{-} \rightarrow \Lambda_{c}^{+} \bar{\Lambda}_{c}^{-} K^{-}\right)=\frac{N_{\text {total }}}{N_{B \bar{B}} \cdot \mathcal{B}^{2}\left(\Lambda_{c}^{+} \rightarrow p^{+} K^{-} \pi^{+}\right) \cdot \sum_{i=1}^{m} f_{i} \cdot \epsilon_{i}}
$$

Similarly,

$$
\mathcal{B}\left(B^{-} \rightarrow \Xi_{c}^{0} \bar{\Lambda}_{c}^{-}\right) \mathcal{B}\left(\Xi_{c}^{0} \rightarrow \Xi^{-} \pi^{+}\right)=\frac{N_{\text {total }}}{N_{B \bar{B}} \cdot \mathcal{B}\left(\Lambda_{c}^{+} \rightarrow p^{+} K^{-} \pi^{+}\right) \cdot \sum_{i=1}^{m} f_{i} \cdot \epsilon_{i}}
$$

with $\mathrm{i}=1,2$.

To ascertain the systematic uncertainty of the combined branching fractions, we repeat the branching fraction calculation by varying the quantities individually within their respective systematic errors for each mode in the case of uncorrelated errors. For correlated errors, the quantities are varied simultaneously for some or all modes together. In this way, the correlations are automatically taken into account. 
In estimating the combined systematics uncertainties, we conservatively assume the PID uncertainties are 100\% correlated in all decay modes, and the uncertainties in the relative branching ratios of the $\Lambda_{c}^{+}$are not correlated. The systematic errors of the combined branching fractions are listed in the Table 8.1.

Table 8.1: The summary of the relative systematic uncertainties(\%) of $B^{-} \rightarrow$ $\Lambda_{c}^{+} \bar{\Lambda}_{c}^{-} K^{-}$and $B^{-} \rightarrow \Xi_{c}^{0} \bar{\Lambda}_{c}^{-}$decays

\begin{tabular}{lcc}
\hline systematic resource & $B^{-} \rightarrow \Lambda_{c}^{+} \bar{\Lambda}_{c}^{-} K^{-}$ & $B^{-} \rightarrow \Xi_{c}^{0} \bar{\Lambda}_{c}^{-}$ \\
\hline \hline$B$ counting & 1.1 & 1.1 \\
Tracking & 9.8 & 10.0 \\
PID & 0.8 & 0.78 \\
MC statistics & 0.75 & 1.58 \\
$\Delta E$ cut & 3 & 3 \\
$\mathcal{B}$ of intermediate state & $3.38 \oplus 52$ & $7.35 \oplus 26$ \\
Fit related & 1.95 & 1.41 \\
\hline total & $11.08 \oplus 52$ & $13.02 \oplus 26$ \\
\hline
\end{tabular}

The combined branching fraction measurements are thus obtained as:

$$
\begin{aligned}
\mathcal{B}\left(B^{-} \rightarrow \Lambda_{c}^{+} \bar{\Lambda}_{c}^{-} K^{-}\right) & =(1.22 \pm 0.16 \pm 0.14 \pm 0.63) \times 10^{-3}(8.16) \\
\mathcal{B}\left(B^{-} \rightarrow \Xi_{c}^{0} \bar{\Lambda}_{c}^{-}\right) \cdot \mathcal{B}\left(\Xi_{c}^{0} \rightarrow \Xi^{-} \pi^{+}\right) & =(2.04 \pm 0.59 \pm 0.26 \pm 0.53) \times 10^{-5}(8.17)
\end{aligned}
$$

The resulting $\chi^{2}$ of the combined $\mathcal{B}\left(B^{-} \rightarrow \Lambda_{c}^{+} \bar{\Lambda}_{c}^{-} K^{-}\right)$is 6.11 for four degree of freedom, and the resulting $\chi^{2}$ of the combined $\mathcal{B}\left(B^{-} \rightarrow \Xi_{c}^{0} \bar{\Lambda}_{c}^{-}\right) \mathcal{B}\left(\Xi_{c}^{0} \rightarrow \Xi^{-} \pi^{+}\right)$is 0.10 for one degree of freedom. This indicates that the BF results obtained from the different modes are in agreement. 


\subsubsection{Method 2 -weighted method}

The Best Linear Unbiased Estimate (BLUE) method [59] is used to combine the branching fractions obtained from different modes and the correlation of the uncertainties are taken into account. The estimate $\hat{\mu}$ of the true observable $\mu$ is constructed as the linear combination of the different measurements as

$$
\hat{\mu}=\sum_{i=1}^{n} w_{i} y_{i}
$$

where $y_{i}$ 's are the measurements of $\mu$, and $w_{i}$ 's are the coefficients to be determined. If the estimate is unbiased, the expectation value of $\hat{\mu}$ should satisfy

$$
E(\hat{\mu})=\sum_{i=1}^{n} w_{i} E\left(y_{i}\right)=\mu, \text { if and only if } \sum_{i=1}^{n} w_{i}=1
$$

The best estimate is obtained by minimizing the variance $V(\hat{\mu})$ of the selected linear combination. The covariance between two measurements $y_{i}$ and $y_{j}$ is denoted as

$$
M_{i j}=\operatorname{cov}\left(y_{i}, y_{j}\right)=\operatorname{cov}\left(y_{j}, y_{i}\right)=M_{j i}
$$

Then the variance of $\hat{\mu}$ can be written as

$$
V(\hat{\mu})=\sum_{i, j} w_{i} M_{i j} w_{j}
$$

We arrive at this with the Lagrange Multiplier method. The Lagrangian function is formed as

$$
S=\sum_{i, j} w_{i} M_{i j} w_{j}+\lambda\left(1-\sum_{i=1}^{n} w_{i}\right)
$$


where $\lambda$ is the Lagrange multiplier. Differentiating $S$ with respect to $w_{i}$ and $\lambda$, we obtain

$$
\left\{\begin{array}{l}
M w=\frac{\lambda}{2} U \\
U^{T} w=1
\end{array}\right.
$$

where $U$ is an n-dimensional vector, each element of $U$ is 1 , and $U^{T}$ is the transpose of $U . M$ is the covariance matrix and $w$ is the coefficient vector. The solutions to these linear equations are easily obtained as

$$
\left\{\begin{array}{l}
\lambda=2\left(U^{T} M^{-1} U\right)^{-1} \\
w=\left(U^{T} M^{-1} U\right)^{-1} M^{-1} U
\end{array}\right.
$$

where $M^{-1}$ is the inverse if $M$. The value and the variance of $\hat{\mu}$ are then

$$
\left\{\begin{array}{l}
\hat{\mu}=y^{T}\left(U^{T} M^{-1} U\right)^{-1} M^{-1} U \\
V(\hat{\mu})=\left(U^{T} M^{-1} U\right)^{-1}
\end{array}\right.
$$

where $y^{T}$ is the transpose of the observable vector $y$.

In combining the results, we assume that there is no correlation between the statistical uncertainties and the systematic uncertainties, and only some of the systematic uncertainties are $100 \%$ correlated between the different $\Lambda_{c}^{+}$final states. The systematic uncertainty from $\mathcal{B}\left(\Lambda_{c}^{+} \rightarrow p^{+} K^{-} \pi^{+}\right)$is not taken into account in the combined calculation, since it is identical for all channels, (and is very large as well).

The combined branching fraction of $B^{-} \rightarrow \Lambda_{c}^{+} \bar{\Lambda}_{c}^{-} K^{-}$obtained using this method is:

$$
\mathcal{B}\left(B^{-} \rightarrow \Lambda_{c}^{+} \bar{\Lambda}_{c}^{-} K^{-}\right)=(1.05 \pm 0.20 \pm 0.55) \times 10^{-3}
$$


where the weights for the five final states in the order stated before are: $0.57,0.05,0.08$, $0.17,0.14$, respectively. The resulting $\chi^{2}$ is 5.00 with four degrees of freedom. And,

$$
\mathcal{B}\left(B^{-} \rightarrow \Xi_{c}^{0} \bar{\Lambda}_{c}^{-}\right) \cdot \mathcal{B}\left(\Xi_{c}^{0} \rightarrow \Xi^{-} \pi^{+}\right)=(2.05 \pm 0.65 \pm 0.53) \times 10^{-5}
$$

where the weights for the two final states in the order stated before are : 0.63 , and 0.37, respectively. The resulting $\chi^{2}$ is 0.09 with one degree of freedom.

Here, the first uncertainty is the combination of the statistical uncertainty and the systematic uncertainty, and the second uncertainty is the uncertainty from the branching fraction of $\mathcal{B}\left(\Lambda_{c}^{+} \rightarrow p^{+} K^{-} \pi^{+}\right)$.

\subsubsection{Comparison of the results from the two methods}

For the three-body $B$ decays, the uncertainties are consistent between these two methods, but the central value from the second method is different from that from the first method. For the two-body decays, both the central values and uncertainties are consistent. However, because the second method does not distinguish between the statistical and the systematic uncertainties, we use the combined results from the first method for our final result.

\subsection{Discussion of the results}

$$
\text { 8.2.1 } B^{-} \rightarrow \Lambda_{c}^{+} \bar{\Lambda}_{c}^{-} K^{-}
$$

Table 8.2.1 shows a comparison between the present results and those obtained by the Belle experiment for the $B^{-} \rightarrow \Lambda_{c}^{+} \bar{\Lambda}_{c}^{-} K^{-}$decay. The branching fractions of $\Lambda^{0}$ and $K_{S}^{0}$ are included in the detection efficiencies, and the overall efficiency is calculated as $\sum_{i, j} \epsilon_{i j}\left(\Gamma_{i} / \Gamma\left(\Lambda_{c}^{+} \rightarrow p^{+} K^{-} \pi^{+}\right)\right)\left(\Gamma_{j} / \Gamma\left(\Lambda_{c}^{+} \rightarrow p^{+} K^{-} \pi^{+}\right)\right)$. The branching 
fraction measured here is much higher than Belle's, with more observed signal events, even though the luminosity used in the present analysis is $40 \%$ lower. The difference is perplexing.

Table 8.2: Summary of luminosities, $B \bar{B}$ pairs, the fitted signal yields, the individual and overall efficiencies (\%) and the branching fractions.

\begin{tabular}{lcc}
\hline \hline & Belle result & BABAR result \\
\hline Luminosity $\left(\mathrm{fb}^{-1}\right)$ & 357 & 207 \\
$B \bar{B}$ pair $\left(10^{6}\right)$ & 386 & 228.3 \\
signal yields & $48.5_{-6.8}^{+7.5}$ & $74.6 \pm 9.8$ \\
Efficiency of $p K \pi, p K \pi(\%)$ & $4.90 \pm 0.06$ & $6.56 \pm 0.08$ \\
Efficiency of $p K \pi, p \bar{K}^{0}(\%)$ & $2.02 \pm 0.04$ & $2.82 \pm 0.03$ \\
Efficiency of $p K^{0}, p K \pi(\%)$ & $2.02 \pm 0.04$ & $2.82 \pm 0.03$ \\
Efficiency of $p K \pi, \bar{\Lambda}^{0} \pi(\%)$ & $2.75 \pm 0.05$ & $3.77 \pm 0.06$ \\
Efficiency of $\Lambda^{0} \pi, p K \pi(\%)$ & $2.75 \pm 0.05$ & $3.83 \pm 0.06$ \\
Overall efficiency $(\%)$ & $7.79 \pm 0.07 \pm 0.24$ & 10.58 \\
\hline BF $\left(\times 10^{4}\right)$ & $6.5_{-0.9}^{+1.0} \pm 0.8 \pm 3.4$ & $12.2 \pm 1.6 \pm 1.4 \pm 6.3$ \\
\hline
\end{tabular}

\subsection{2 $B^{-} \rightarrow \Xi_{c}^{0} \bar{\Lambda}_{c}^{-}$}

Table 8.2.2 shows a comparison between the present results and those from the Belle experiment for the $B$ decay final states including a $\Xi_{c}^{0}$. Here, the observed signals are small with large uncertainties, making meaningful comparison difficult. However, we compare the present exclusive measurement with our previous inclusive measurement of $B \rightarrow \Xi_{c}^{0} X$, namely, $\mathcal{B}\left(B \rightarrow \Xi_{c}^{0} X\right) \mathcal{B}\left(\Xi_{c}^{0} \rightarrow \Xi^{-} \pi^{+}\right)=(2.11 \pm 0.19 \pm$ $0.25) \times 10^{-4}$. This, in turn, yields $\mathcal{B}\left(B^{-} \rightarrow \Xi_{c}^{0} \bar{\Lambda}_{c}^{-}\right) / \mathcal{B}\left(B \rightarrow \Xi_{c}^{0} X\right)=(9.0 \pm 3.9) \%$. 
Table 8.3: Summary of luminosities, $B \bar{B}$ pairs, the fitted signal yields, the overall efficiencies and the products of branching fractions

\begin{tabular}{|c|c|c|c|c|}
\hline & \multicolumn{2}{|c|}{$B^{-} \rightarrow \Xi_{c}^{0} \bar{\Lambda}_{c}^{-}, \Xi_{c}^{0} \rightarrow \Xi^{-} \pi^{+}$} & \multicolumn{2}{|c|}{$B^{-} \rightarrow \Xi_{c}^{0} \bar{\Lambda}_{c}^{-}, \Xi_{c}^{0} \rightarrow \Lambda^{0} K^{-} \pi^{+}$} \\
\hline & Belle result & $B A B A R$ & Belle's result & $B A B A R$ \\
\hline $\operatorname{Lum}\left(\mathrm{fb}^{-1}\right)$ & 357 & 207 & 357 & 207 \\
\hline$B \bar{B} \operatorname{pair}\left(10^{6}\right)$ & 386 & 228.3 & 386 & 228.3 \\
\hline signal Yield & $12.4_{-3.3}^{+4.2}$ & $6 \pm 2.4$ & $16.9_{-4.0}^{+4.8}$ & $7.5 \pm 3.1$ \\
\hline Efficiency $(\%)$ & 1.14 & 2.76 & 2.04 & 2.86 \\
\hline $\mathcal{B}\left(10^{-5}\right)$ & $5.6_{-1.5}^{+1.9} \pm 0.8 \pm 1.5$ & $1.91 \pm 0.77 \pm 0.20 \pm 0.49$ & $4.0_{-0.9}^{+1.1} \pm 0.8 \pm 1.0$ & $2.46 \pm 1.02 \pm 0.26 \pm 0.64$ \\
\hline
\end{tabular}


An early phenomenology paper $[28]$ predicts $\mathcal{B}\left(B^{-} \rightarrow \bar{\Sigma}_{c}^{--} \Xi_{c}^{+}\right): \mathcal{B}\left(B^{-} \rightarrow \bar{\Sigma}_{c}^{-} \Xi_{c}^{0}\right)$ : $\mathcal{B}\left(B^{-} \rightarrow{\overline{\Xi_{c}^{0}}}_{c}^{+}\right)=4: 2: 1$. a substantially higher $b \rightarrow c \bar{c} s$ contribution in the $B \rightarrow$ $\Xi_{c} X$ decays. In the current measurement, an enhancement in the $\Delta E<0.05 \mathrm{GeV}$ region is observed, which might originate from the decays $B^{-} \rightarrow \Xi_{c}^{0} \bar{\Lambda}_{c}^{-}+m \pi(\gamma)$. This can also indicate that the $b \rightarrow c \bar{c} s$ process is the dominant mechanism in the $B \rightarrow$ $\Xi_{c} X$ decays. However, the uncertainty in our measurement is too big to draw any significant conclusion. Such quantitative conclusions will have to await the expected $1 \mathrm{ab}^{-1}$ of data.

\subsection{Study of intermediate resonances in $B^{-} \rightarrow \Lambda_{c}^{+} \bar{\Lambda}_{c}^{-} K^{-}$decay}

Figure 8.1 shows the Dalitz plots for $B^{-} \rightarrow \Lambda_{c}^{+} \bar{\Lambda}_{c}^{-} K^{-}\left(\Lambda_{c}^{+} \rightarrow p^{+} K^{-} \pi^{+}\right.$for $\Lambda_{c}^{+}$ and $\bar{\Lambda}_{c}^{-}$) decay. Here, all the selection cuts are applied and only the events in the signal region of $5.27 \mathrm{GeV}<m_{\mathrm{ES}}<5.29 \mathrm{GeV}$ are plotted. Figure 8.2 shows the projections onto (a) $m\left(\Lambda_{c}^{+} \bar{\Lambda}_{c}^{-}\right)\left(\Lambda_{c}^{+}\right.$and $\bar{\Lambda}_{c}^{-}$are suggested to decayed from charmomium, not directly from $B$ meson [33]), (b) $m\left(\Lambda_{c}^{+} K^{-}\right)$and (c) $m\left(\bar{\Lambda}_{c}^{-} K^{-}\right)$for the same events used in Fig. 8.1. In the projection plots, the events distributions from the signal MC are scaled by the ratio of signal yield from signal MC and from data. The background distribution in the sideband region of $m_{\mathrm{ES}}<5.26 \mathrm{GeV}$ from data is scaled to the estimated background yield in the signal region. In the Dalitz plot for data, an accumulation of events is observed in the region where $m\left(\Lambda_{c}^{+} K^{-}\right)>2.9 \mathrm{GeV}$. This accumulation is not present in the signal $\mathrm{MC}$ or in $m_{\mathrm{ES}}$ sideband of in data, and it could imply presence of some intermediate resonance states, e.g. $\Xi_{c}^{*} \rightarrow \Lambda_{c}^{+} K^{-}$. But 
the data sample is simply too small to draw any conclusion.
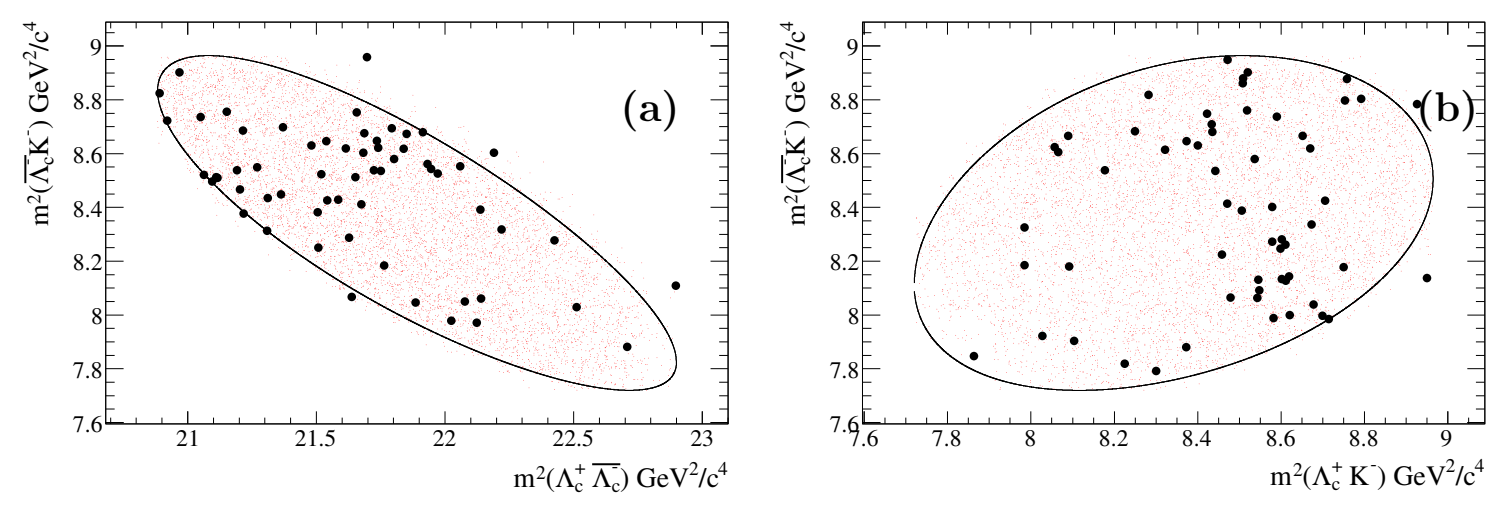

Figure 8.1: The Dalitz Distribution of $B^{-} \rightarrow \Lambda_{c}^{+} \bar{\Lambda}_{c}^{-} K^{-}$in the signal region $5.27 \mathrm{GeV}<m_{\mathrm{ES}}<5.29 \mathrm{GeV}$, where $\Lambda_{c}^{+} \rightarrow p^{+} K^{-} \pi^{+}$. (a) $m^{2}\left(\bar{\Lambda}_{c}^{-} K^{-}\right)$vs. $m^{2}\left(\Lambda_{c}^{+} \bar{\Lambda}_{c}^{-}\right)$, (b) $m^{2}\left(\bar{\Lambda}_{c}^{-} K^{-}\right)$vs. $m^{2}\left(\Lambda_{c}^{+} K^{-}\right)$. The large black points are the events reconstructed from data, and the small red points are the reconstructed signal events generated with a phase space model. The black curves are the boundary of the Dalitz plots restricted by the four-momentum conservation. Mass constraints on $B$ is not applied, so points can lie outside the boundary.

Excited $\Xi_{c}$ states are known to exist in this mass range $(\sim 2930 \mathrm{MeV})$, but phenomenological predictions alone can not be depended upon. Several states[57],[58] might be present with small predicted mass splittings; thus making it hard to compare these models to the data. 

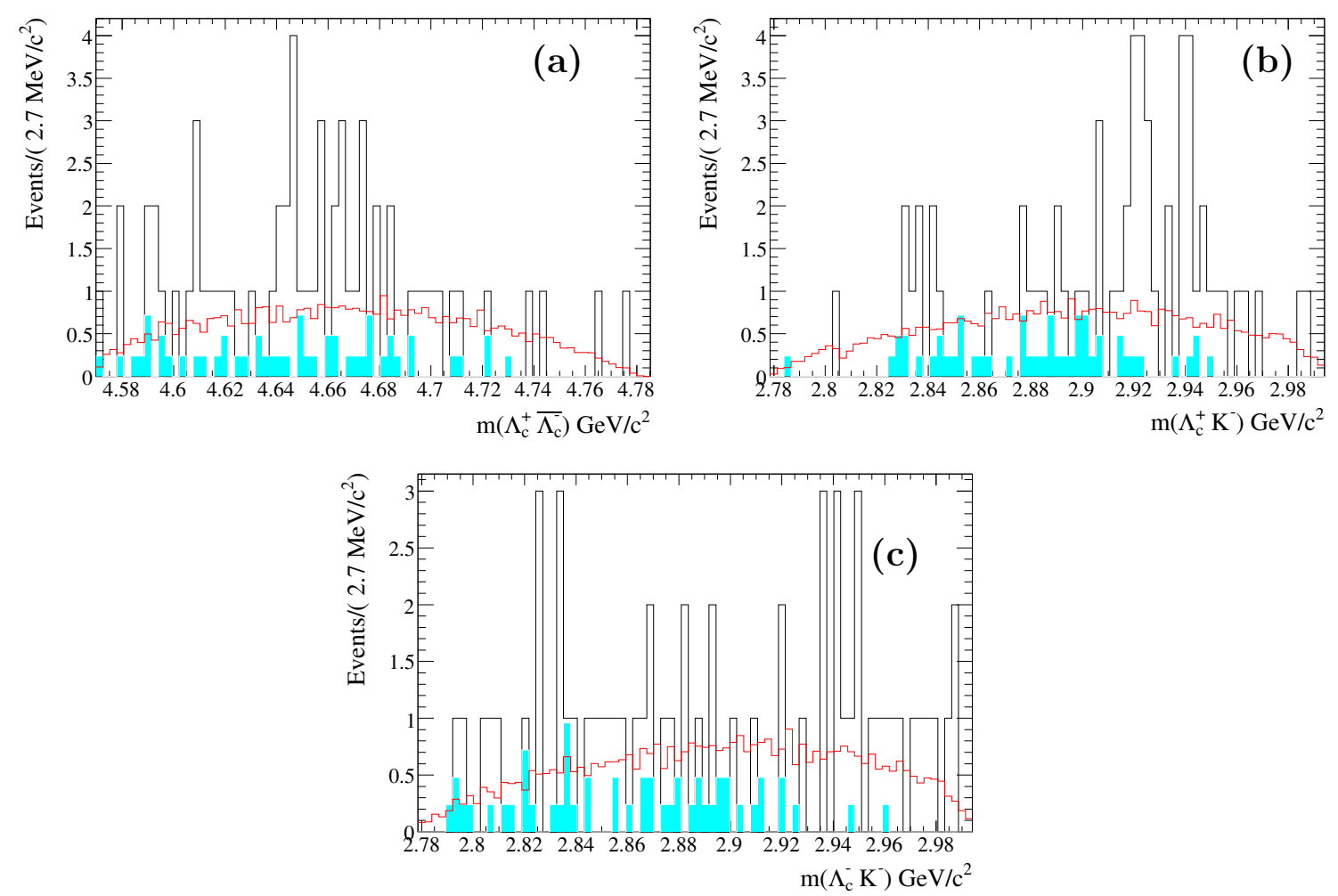

Figure 8.2: The projections $m\left(\Lambda_{c}^{+} \bar{\Lambda}_{c}^{-}\right)$(a), $m\left(\bar{\Lambda}_{c}^{-} K^{-}\right)$(b) and $m\left(\Lambda_{c}^{+} K^{+}\right)$(c) for the same events used in fig 8.1. The black curve is reconstructed from data, the red curve represents the distribution of reconstructed signal events generated with a phase space model, and the blue hatched region represents the background distribution estimated from the events in the region of $m_{\mathrm{ES}}<5.26 \mathrm{GeV}$. 


\section{CHAPTER 9 \\ SUMMARY AND OUTLOOK}

This thesis documents measurements of the branching fractions of exclusive $B$ meson decays into final states with two charmed baryons using $228 \mathrm{fb}^{-1} B A B A R$ data collected between 1999 and 2004. The measured branching fractions are:

$$
\begin{aligned}
\mathcal{B}\left(B^{-} \rightarrow \Lambda_{c}^{+} \bar{\Lambda}_{c}^{-} K^{-}\right) & =(1.22 \pm 0.16 \pm 0.14 \pm 0.63) \times 10^{-3} \\
\mathcal{B}\left(\bar{B}^{0} \rightarrow \Lambda_{c}^{+} \bar{\Lambda}_{c}^{-} \bar{K}^{0}\right) & =(0.40 \pm 0.33 \pm 0.05 \pm 0.21) \times 10^{-3} \\
\mathcal{B}\left(B^{-} \rightarrow \Xi_{c}^{0} \bar{\Lambda}_{c}^{-}\right) \cdot \mathcal{B}\left(\Xi_{c}^{0} \rightarrow \Xi^{-} \pi^{+}\right) & =(2.04 \pm 0.59 \pm 0.26 \pm 0.53) \times 10^{-5} \\
\mathcal{B}\left(\bar{B}^{0} \rightarrow \Xi_{c}^{+} \bar{\Lambda}_{c}^{-}\right) \times \mathcal{B}\left(\Xi_{c}^{+} \rightarrow \Xi^{-} \pi^{+} \pi^{+}\right) & =(1.50 \pm 1.07 \pm 0.19 \pm 0.39) \times 10^{-5}
\end{aligned}
$$

where the first uncertainty is statistical, the second is systematic, and the third is due to the uncertainty on the branching fraction of $B^{-} \rightarrow \Lambda_{c}^{+} \bar{\Lambda}_{c}^{-} K^{-}$.

The analysis is still statistically limited, especially for the two neutral $B$ decay modes. By the time BABAR shuts down at the end of 2008, the experiment would accumulate approximately four times as much data as that used in this analysis, reducing the statistical uncertainties to half of those obtained here.

The systematic uncertainties are dominated by the uncertainty from the absolute branching fraction of $\Lambda_{c}^{+} \rightarrow p^{+} K^{-} \pi^{+}: 50 \%$ for $\bar{B} \rightarrow \Lambda_{c}^{+} \bar{\Lambda}_{c}^{-} \bar{K}$ decays, and $25 \%$ for the $\bar{B} \rightarrow \Xi_{c} \bar{\Lambda}_{c}^{-}$decays. A more accurate measurement of $\mathcal{B}\left(\Lambda_{c}^{+} \rightarrow p^{+} K^{-} \pi^{+}\right)$is needed. The best published value [60] of $\mathcal{B}\left(\Lambda_{c}^{+} \rightarrow p^{+} K^{-} \pi^{+}\right)$has been inferred from continuum charm production $e^{+} e^{-} \rightarrow \Lambda_{c}^{+} X$ with $3.1 \mathrm{fb}^{-1}$ of data collected by CLEO in 2000. With much more data BABAR can make a more accurate measurement. 
Also, the absolute branching fraction of $\Xi_{c}$ will likely be available [61][60], which in turn will make the measurement of the absolute branching fractions of $\bar{B} \rightarrow \Xi_{c} \bar{\Lambda}_{c}^{-}$ possible.

The branching fraction of the decay $B^{-} \rightarrow \Lambda_{c}^{+} \bar{\Lambda}_{c}^{-} K^{-}$is expected to be highly suppressed by the tiny phase space available, but our measurement and BELLE's measurement are both three orders of magnitude higher than this naive expectation. In this analysis, we examined the distribution of the two-body invariant masses $m\left(\Lambda_{c}^{+} K^{-}\right)$and $m\left(\Lambda_{c}^{+} \bar{\Lambda}_{c}^{-}\right)$in an effort to explain the difference. However, the limited statistics prevent us from making any quantitative conclusion at this point. With about 300 signal events expected with the complete $B A B A R$ data sample available in 2008, one hopes to be able to draw some firm conclusions at this point. The current experimental measurements raise a lot of questions regarding the validity of some of the theoretical/phenomenological assumptions involving various technical methods used in non-perturbative estimates. Further detailed and accurate measurements will hopefully shed light into these areas, which need to be understood better. 


\section{REFERENCES}

[1] D. J. Griffiths, NEW YORK, USA: WILEY (1987) 392p

[2] M. Gell-Mann, Phys. Lett. 8, 214 (1964).

[3] G. Zweig, CERN-TH-401, 26 (1964).

[4] D. Diakonov, V. Petrov and M. V. Polyakov, Z. Phys. A 359, 305 (1997)

[5] C. Alt et al. [NA49 Collaboration], Phys. Rev. Lett. 92, 042003 (2004)

[6] T. Nakano et al. [LEPS Collaboration], Phys. Rev. Lett. 91, 012002 (2003)

[7] Y. Fukuda et al. [Super-Kamiokande Collaboration], Phys. Rev. Lett. 82, 2644 (1999)

[8] Q. R. Ahmad et al. [SNO Collaboration], Phys. Rev. Lett. 87, 071301 (2001)

[9] A. Astbury, Phys. Bull. 34, 434 (1983).

[10] W. Bartel et al. [JADE Collaboration], Phys. Lett. B 91, 142 (1980).

[11] N. Cabibbo, Phys. Rev. Lett. 10, 531 (1963).

[12] M. Kobaya shi and T. Maskawa, Prog. Theor. Phys. 49, 652 (1973).

[13] L. Wolfenstein, Phys. Rev. Lett. 51, 1945 (1983).

[14] R. N. Cahn, Rev. Mod. Phys. 68, 951 (1996).

[15] P. F. . Harrison and H. R. . Quinn [BABAR Collaboration],, SLAC-R-504 (1998).

[16] M. K. Gaillard, B. W. Lee and J. L. Rosner, Rev. Mod. Phys. 47, 277 (1975).

[17] J. J. Aubert et al. [E598 Collaboration], Phys. Rev. Lett. 33, 1404 (1974).

[18] J. E. Augustin et al. [SLAC-SP-017 Collaboration], Phys. Rev. Lett. 33, 1406 (1974).

[19] G. Goldhaber et al., Phys. Rev. Lett. 37, 255 (1976). 
[20] I. Peruzzi et al., Phys. Rev. Lett. 37, 569 (1976).

[21] E. G. Cazzoli et al., Phys. Rev. Lett. 34, 1125 (1975).

[22] S. J. Brodsky and A. H. Mueller, Phys. Lett. B 206, 685 (1988).

[23] K. G. Wilson, Phys. Rev. 179 (1969) 1499.

[24] A. V. Radyushkin, arXiv:hep-ph/0101227.

[25] N. Isgur and M. B. Wise, Phys. Lett. B 232, 113 (1989).

[26] Phys. Rev. D 70, 091106, 2004

[27] B. Aubert et al. [BABAR Collaboration], Phys. Rev. Lett. 95, 142003 (2005)

[28] V. L. Chernyak and I. R. Zhitnitsky, Nucl. Phys. B 345, 137 (1990).

[29] P. Ball and H. G. Dosch, Z. Phys. C 51, 445 (1991).

[30] K. Abe et al. [Belle Collaboration], Phys. Rev. Lett. 97, 202003 (2006)

[31] R. Chistov et al., Phys. Rev. D 74, 111105 (2006)

[32] H. Y. Cheng and K. C. Yang, Phys. Rev. D 66, 014020 (2002) [33]

[33] H. Y. Cheng, C. K. Chua and S. Y. Tsai, arXiv:hep-ph/0512335.

[34] C. H. Chen, Phys. Lett. B 638, 214 (2006)

[35] J. R. Rees [LBL-SLAC Joint PEP Group], IEEE Trans. Nucl. Sci. 24, 1836 (1977).

[36] http://www.lns.cornell.edu/public/lab-info/upsilon.html

[37] The BABAR Collaboration, Nuclear Instruments and Methods A479, 1 (2002).

[38] W. Menges, arXiv:physics/0609039.

[39] A. Berenyi et al., IEEE Transactions on Nuclear Sciense, 46, 6, 2006 (1999).

[40] A. Berenyi et al., IEEE Transactions on Nuclear Sciense, 46, 353 (1999).

[41] A. Berenyi et al., IEEE Transactions on Nuclear Sciense, 46, 928 (1999). 
[42] P.V.C. Hough, U.S. Patent 3069654, 1962.

[43] P. Billoir, Nucl. Instr. Methods Phys. Res., Sect. A 225 (1984) 225.

[44] D.N. Brown, E.A. Charles, D.A. Roberts, The BABAR Track Fitting Algorithm, Proceedings of CHEP 2000, Padova, Italy (2000).

[45] W. D. Hulsbergen, Nucl. Instrum. Meth. A 552, 566 (2005)

[46] S. Agostinelli et al. [GEANT4 Collaboration], Nucl. Instrum. Meth. A 506, 250 (2003).

[47] S. Eidelman et al. [Particle Data Group], Phys. Lett. B 592, 1 (2004).

[48] T. Lesiak et al. [BELLE Collaboration], Phys. Lett. B 605, 237 (2005) [Erratumibid. B 617, 198 (2005)]

[49] B. Aubert et al. [BABAR Collaboration], Phys. Rev. D 75, 052002 (2007)

[50] P. Golonka and Z. Was, arXiv:hep-ph/0604232.

[51] Tracking Efficiency Task Force, http://www.slac.stanford.edu/BFROOT/www/Physics/

TrackEfficTaskForce/TrackingTaskForce-2004.html

[52] http://www.slac.stanford.edu/BFROOT/www/Physics/Tools/Pid/PidOnMc/ pidonmc.html

[53] F. James and M. Roos, Comput. Phys. Commun. 10, 343 (1975).

[54] R. Brun, F. Rademakers, Linux Journal, 1998, Issue 51, Metro Link Inc.

[55] http://roofit.sourceforge.net

[56] Christopher Hearty, "Measurement of the Number of Upsilon(4S) Mesons Produced in Run 1 (B Counting)", BAD 134, 2001

[57] K. Maltman and N. Isgur, Phys. Rev. D 22, 1701 (1980).

[58] L. Y. Glozman and D. O. Riska, Nucl. Phys. A 603, 326 (1996) [Erratum-ibid. A 620, 510 (1997)]

[59] A. Valassi, Nucl. Instrum. Meth. A 500, 391 (2003). 
[60] D. E. Jaffe et al. [CLEO Collaboration], Phys. Rev. D 62, 072005 (2000)

[61] I. I. Bigi, Eur. Phys. J. C 24, 271 (2002) 